\title{
35. A NEW RADIOLARIAN BIOSTRATIGRAPHY FOR THE NEOGENE OF THE NORWEGIAN SEA: ODP LEG 1041
}

\author{
Robert M. Goll ${ }^{2}$ and Kjell R. Bjørklund ${ }^{3,4}$
}

\begin{abstract}
Radiolaria are present in frequencies ranging from rare to abundant and with generally moderate to good preservation quality in Leg 104 sediments younger than $22 \mathrm{Ma}$. Preservation degrades in progressively younger sediments, and upper Pliocene to mid-Pleistocene radiolaria were found only at Site 644, where sporadic assemblages of moderate to poorly preserved specimens persist to approximately $0.75 \mathrm{Ma}$. Radiolaria are essentially absent in Leg 104 recovery older than basal Miocene.

The stratigraphic ranges of 55 taxa of radiolaria are documented in 451 samples from the biosiliceous recoveries of Holes $642 \mathrm{~B}, 642 \mathrm{C}, 642 \mathrm{D}, 643 \mathrm{~A}$, and $644 \mathrm{~A}$. The stratigraphic ranges of 25 of these species are used as boundary criteria for a new system of 28 Neogene zones and subzones that are used to characterize approximately $72 \%$ of the past $22 \mathrm{~m}$.y. of sedimentation on the Vøring Plateau. This new scheme is intended to supercede the NRS zones provisionally proposed in the Leg 104 Initial Reports. The applicability of this regional biozonation beyond the Vøring and Iceland Plateaus is not presently known.

The radiolaria biostratigraphy serves as a basis for inferring a sequence of hiatuses and faunal overturns that may be associated with sea-level low stands and consequent cold-water isolation of the Norwegian Sea.

Twenty-one new taxa are described as follows: Actinomma henningsmoeni, Actinomma livae, Actinomma mirabile, Actinomma plasticum, Ceratocyrtis broeggeri, Ceratocyrtis manumi, Ceratocyrtis stoermeri, Clathrospyris vogti, Corythospyris hispida, Corythospyris jubata sverdrupi, Corythospyris reuschi, Crytocapsella ampullacea, Cyrocapsella kladaros, Gondwanaria japonica kiaeri, Hexalonche esmarki, Larcospira bulbosa, Phormospyris thespios, Pseudodicytophimus amundseni, Spongotrochus vitabilis, Spongurus cauleti, and Tessarastrum thiedei.
\end{abstract}

\section{INTRODUCTION}

Sediments recovered on Leg 104 have been the focus of intense biostratigraphic examinations by many specialists, and this activity will undoubtedly continue well beyond the publication of this volume. In terms of radiolarian research, this paper can only serve as a foundation for more detailed future studies of these Neogene high-latitude faunas. Although both abundance and preservation quality of radiolarians in Leg 104 sediments vary considerably, these microfossils are generally numerous and moderate to well preserved throughout the pelagic facies penetrated at Sites 642 and 643, which range in age from 3 to $22 \mathrm{Ma}$. Radiolarians are very rare or absent in the younger glacial marine sequences at these sites, but siliceous microfossils persist sporadically in Hole 644A sediments up to an age of approximately $0.75 \mathrm{Ma}$. The modern radiolarian fauna reappears in the Holocene surface layer of Hole 642A. As a result of the long continuously-cored holes drilled on Leg 104, we now have a much improved perspective of the Neogene radiolaria biostratigraphy of this subarctic region than was previously available based on DSDP Leg 38 recovery alone.

From the results of DSDP Leg 38 and ODP Leg 104, the complex history of Cenozoic sedimentation on the Vøring Plateau is unfolding. Long intervals of continous sedimentation have not been characteristic of the Vøring Plateau and perhaps not of the Norwegian Sea in general. On the contrary, the longest interval of uninterrupted sedimentation in the Leg 104 recovery is only $3.0 \mathrm{~m}$.y. (Cores $104-643 \mathrm{~A}-15 \mathrm{H}$ to $-30 \mathrm{X}$; see Goll, this

\footnotetext{
${ }^{1}$ Eldholm, O., Thiede, J., Taylor, E., et al., 1989. Proc. ODP, Sci. Results, 104: College Station, TX (Ocean Drilling Program).

2 IKU Sintef, Postbox 1883 Jarlesletta, N-7002 Trondheim, Norway.

${ }^{3}$ Department of Geology, Section B, University of Bergen, Allegaten 41, N5000 Bergen, Norway.

${ }^{4}$ Present address: Paleontological Museum, University of Oslo, Sarsgt.1, N0562 Oslo 5, Norway.
}

volume), and there are examples of hiatus-bounded sections representing only $0.2 \mathrm{~m} . \mathrm{y}$. of sedimentation. Fortunately, most of these hiatuses are only of local extent, and sediments coeval to missing sections at one site have been recovered at one or more of the other sites. There are at least two major breaks in the $\mathrm{Ne}$ ogene sedimentary record as recorded in Leg 104 recovery which may represent unconformities of regional magnitude (10.2-13.4 $\mathrm{Ma}$ and 19.3-21.0 Ma), although it appears that sediments cored in Holes 338,341 , and 348 at least partially fill these gaps. We will argue in the following discussion that a thin layer of sediments associated with the younger of these two major hiatuses may be incorrectly dated in the biostratigraphic synthesis chapter (Goll, this volume). Nevertheless, we have adopted throughout the present paper the core ages presented in the synthesis. Even considering the recoveries from both Legs 38 and 104 however, there is only an imprecise and incomplete record of radiolarian biostratigraphy in the Norwegian Sea at the Oligocene/Miocene boundary (23.6 Ma according to Berggren et al., 1985 ) or during the earliest 2 m.y. of Miocene time. Within these limitations, we have constructed a synthetic composite section of radiolarian-bearing sediments from the Vøring Plateau based on Leg 104 recovery alone, that is approximately $72 \%$ complete for the past $22 \mathrm{Ma}$.

A total of 55 radiolarian taxa have been documented in 451 samples from Holes 642B, 642C, 642D, 643A, and 644A (see Fig. 1). Because of the time constraint imposed on this study and the magnitude of the work, only a small percentage of the total fauna has been included in this report. The ranges of many of the species proposed by Bjørklund (1976), Dzinoridze et al. (1978), and Petrushevskaya and Kozlova (1979) have not been documented here, and this important work remains for future studies. Because our most immediate objective has been to elaborate a radiolarian biozonation that can be used to correlate Leg 104 sediments, our attention has been concentrated on elements of these Neogene faunas that were anticipated to have biostratigraphic value. Therefore, we have selected a small subset of species with 


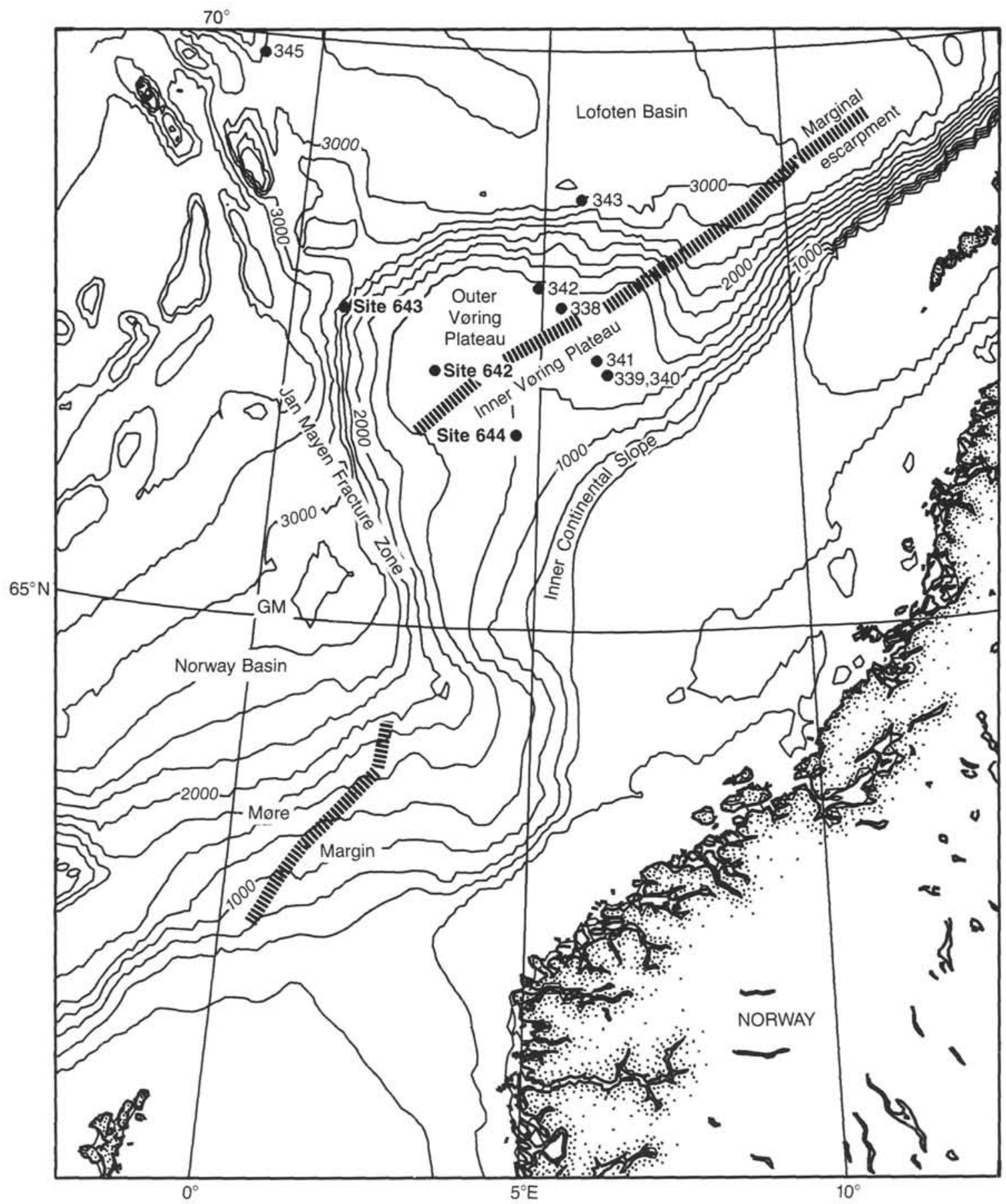

Figure 1. Location map of ODP Sites $642,643,644$, and bathymetry of the Vøring Plateau region of the Norwegian-Greenland Sea. Contours, $250 \mathrm{~m}$.

easily distinguishable morphologies and well-defined ranges. Some of the species included in this study have proven to have low biostratigraphic utility, and only 25 species are employed as zonal marker criteria. Additionally, some longer ranging species which undergo pronounced abundance variations have also been included in this assemblage. On this basis, a system of 28 zones and subzones are proposed to characterize the Neogene pelagic sediments of the Vøring Plateau recovered on Leg 104.

Marked provincialism appears to have been a characteristic of Norwegian Sea radiolarians throughout much of the Neogene. Under the current regimes of the modern ocean, arctic/ subarctic species are swept southward into the western margin of the Norwegian Sea by the East Greenland Current, and a subfauna of temperate species follow the North Atlantic Current into the eastern basins of the Norwegian Sea. Presumably, similar current patterns have influenced radiolarian distributions during much of the Neogene, but there appear to have been intervals of time during which temperate populations have been largely excluded from this region, and cold-water species were dominant.

The modern radiolarian fauna restricted to true arctic regions is only poorly known, and Tertiary arctic radiolaria fau- 
nas are virtually unknown. As a consequence, the biosiliceous sediments of the Norwegian Sea provide a rare, and perhaps unique, opportunity to study cold-water, high northern latitude radiolarians, although it is not presently possible to determine whether these species represent elements of the arctic fauna or whether they are Norwegian Sea endemics. Phormospyris knutheieri (Goll and Bjørklund, 1987) is an example of a modern Norwegian Sea endemic radiolarian species with strong affinities to the North Pacific fauna. There is no evidence that this species is transported southward through the Denmark Strait into the western North Atlantic. As in the present oceans, the latitudinal aseismic ridges bordering the southern margin of the Norwegian Sea have apparently served as an effective barrier restricting free interchange for large portions of the radiolarian faunas throughout the Neogene. The reasons for this isolation are not clearly understood. Either many of these radiolarians are sufficiently deep living to be physically constrained by these ridges, or they are physiologically sensitive to fine-scale changes in water-mass properties.

On the basis of our present investigation, there appear to have been numerous abrupt changes in the species composition of the radiolarian faunas of the Vøring Plateau during the Neogene. Species appear throughout the composite section without obvious indigenous antecedents. In part, these appearances may have resulted from rapid local evolution which has not been detected during these preliminary studies, but more probably these fluctuations represent rapid readjustments in faunal composition induced by changes in current regimes and water masses. In Leg 104 recovery, abrupt shifts in faunal composition attributable to these causes are easily confused with faunal changes associated with hiatuses.

Because of faunal dissimilarities and other problems discussed above, the temperate to tropical Cenozoic radiolarian biozonation (Sanfilippo et al., 1985) is not applicable to Neogene pelagic sediments of the Norwegian Sea. Additionally, it has not been possible to utilize the North Pacific biozonation (Fig. 2), which might be assumed to be a plausible substitute. This latter scheme differs from the tropical biozonation only in the five Pliocene and Pleistocene zones, although age assignments for the common Miocene zones differ significantly (compare Sakai, 1984 with Berggren et al., 1985 for ages of the Sitchocorys peregrina to the Lychocanoma elongata Zones). Under these circumstances, a local biostratigraphic scheme must be constructed that can be compared to magnetic polarity stratigraphy and other biozonations presumed to have more reliable chronologic control. The system of 28 zones and subzones proposed in this paper represent sweeping changes in the previous radiolaria biostratigraphies for this region. Numerical ages for the upper Miocene and higher zones can be assigned with reasonable confidence because of interpretable polarity records and the presence of calcareous microfossils. Ages for the middle and lower Miocene zones are less reliable, because calcareous microfossils have very sporadic occurrences and the polarity records for this interval do not lend themselves to simple interpretation. Integration of biostratigraphic data on radiolarian distributions in both Leg 38 and Leg 104 sediments is at a preliminary stage at this writing, and only the general relationships of these recoveries are discussed in this paper (Fig. 2).

The geographic limits of the present biozonation are indeterminate at this time. With the exception of Site 348 on the Iceland Plateau, all of the Neogene biosiliceous sediments recovered from the Norwegian Sea are from the Vøring Plateau. Although this biozonation is applicable to all Leg 38 and Leg 104 Neogene biosiliceous sediment, we have no means to determine the latitudinal extent of its application, but it must be regarded as a high northern latitude regional scheme.

\section{METHODS}

One sample per section and the core catcher of each core were examined in the biosiliceous intervals of Holes 642B, 642C, 642D, 643A, and $644 \mathrm{~A}$. The noncalcareous, coarse-clastic fractions $(>45 \mu \mathrm{m})$ were separated from bulk sediment using routine laboratory procedures and mounted as simple strewn slides in balsam under $22 \times 50 \mathrm{~mm}$ cover glass. Visual estimates of preservation quality and radiolarian abundance as a function of total clastic composition were made for each sample. Radiolarian preservation is recorded as good $(G)$ if the majority of the specimens are whole and retain fine structural details. Moderate preservation (M) indicates a higher frequency of broken specimens lacking such structural details. Poor preservation $(\mathrm{P})$ is recorded if the majority of radiolaria specimens are fragmentary or have a generally "corroded" aspect. Radiolarian abundance was estimated by noting the average number of specimens observed in $4.9 \mathrm{~mm}^{2}$ fields-of-view of a stereo microscope using the numerical criteria stipulated in Table 1.

Abundances of each species were estimated for each sample by averaging the total number of specimens observed on a horizontal scan traverse of $50 \mathrm{~mm}$ under a compound microscope at a magnification of $\times 100$ using criteria shown in Table 1 . Frequencies of nonradiolarian particles in the residues were not taken into account by this technique, and the reader is cautioned that species recorded as abundant in samples with few radiolarians have much higher frequencies of occurrence within the radiolarians assemblage than species designated as abundant in samples with abundant radiolaria. Tabulations of the semiquantitative frequencies of occurrence of these species are presented in Tables 2, 3, 4, 5 and, 6 .

Type specimens for each of the 21 new taxa have been deposited in the Paleontological Museum, Oslo. Under SYSTEMATIC DESCRIPTIONS, the six digit numbers prefixed by PMO are the reference designations of that repository.

\section{BIOZONATION}

A provisional system of 13 radiolarian zones (NSR1-NSR13) was employed to characterize the Leg 104 cores (Eldholm, Thiede, Taylor, et al., 1987) but regrettably these units were not adequately defined (p. 39). Zones NSRl, NSR2, NSR6, NSR7, NSR 12 and NSR13 were essentially the Velicuccullus oddgurneri Zone, Gondwanaria japonica Zone, Actinomma holtedahli Zone, Lithomelissa stigi Zone, Antarctissa whitei Zone and Cycladophora davisiana Zone, respectively, of Bjørklund (1976). Zone NSR8 was the Eucoronis fridtjofnanseni Zone of Goll and Bjørklund (1980). The basal boundary criteria for Zones NSR3, NSR4, NSR10 and NSR11 were identical to those of the Clathrosphyris sandellae Zone, Pseudodictyophimus amundseni Zone, Tessarastrum thiedei Zone, and Liriospyris cricus Zone, respectively, of this report. The first appearances of Actinomma livae, and Pterocanium korotnevi defined the bases of Zones NSR5 and NSR9, respectively. The Leg 104 Initial Reports biozonation has proven to be inadequate for two important reasons. Thick sedimentary sequences representing long intervals of sedimentation without coeval facies equivalents in Leg 38 material were recovered on Leg 104, and new zones are proposed to accomodate these sediments. Moreover, our more recent studies indicate that some of the NSR zones are untenable, because the species employed as boundary criteria have sporadic distributions ranging over much broader intervals than previously anticipated. This latter case is particularly obvious for NSR6 ( $A c$ tinomma holtedahli Zone), the nominate species of which has been found to range as low as Section 104-642-D-11X-1. Similarly, A.livae and $P$. korotnevi have proven to be unreliable zonal boundary criteria. Problems such as these resulted in highly diachronous correlations for the middle-lower Miocene (Fig. 2).

Our original objective was to formulate a system of perhaps 10 units based exclusively on the first occurrences of consecutively overlapping species. However, the particular nature of radiolarian biostratigraphy in the Norwegian Sea has imposed the 


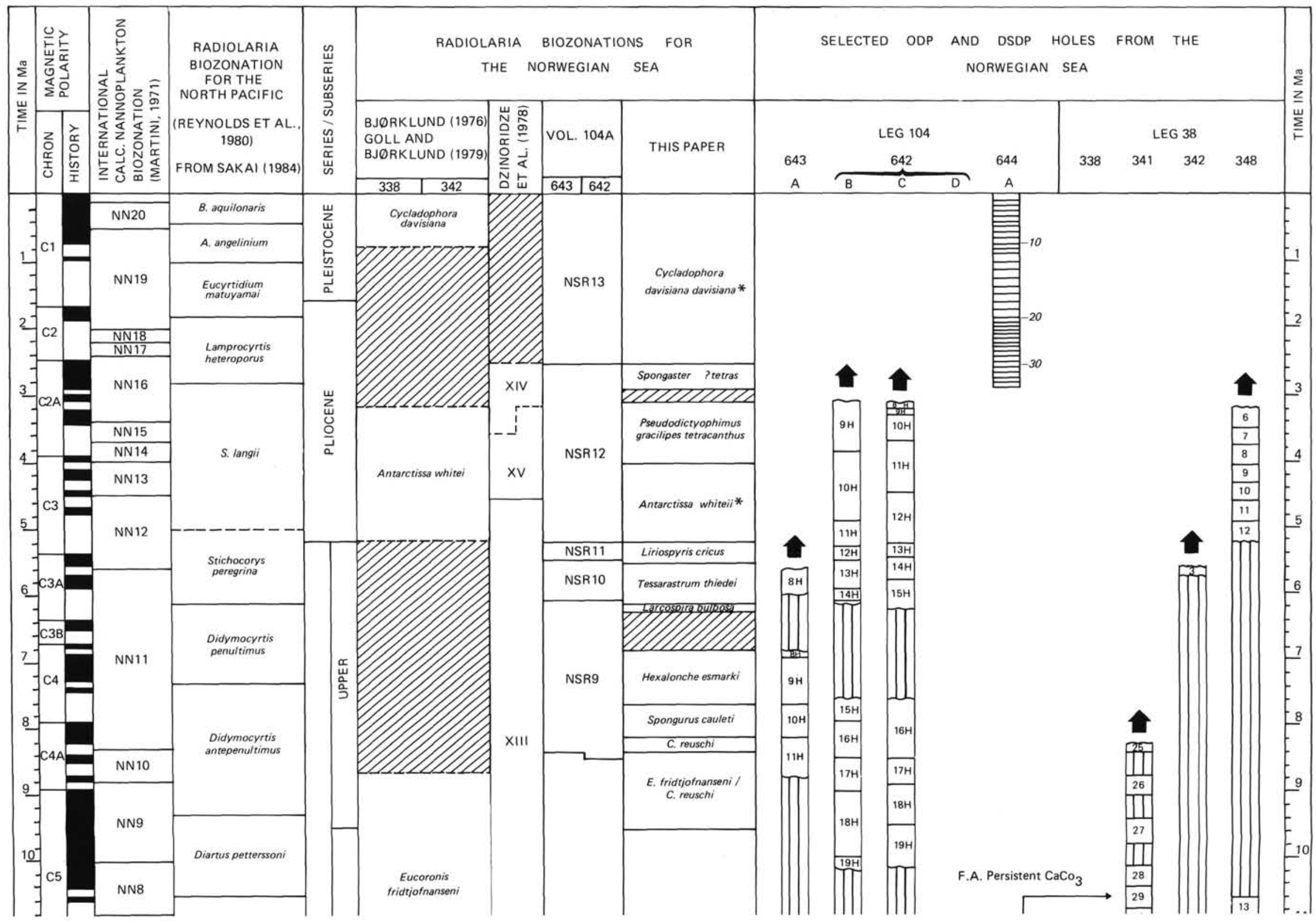




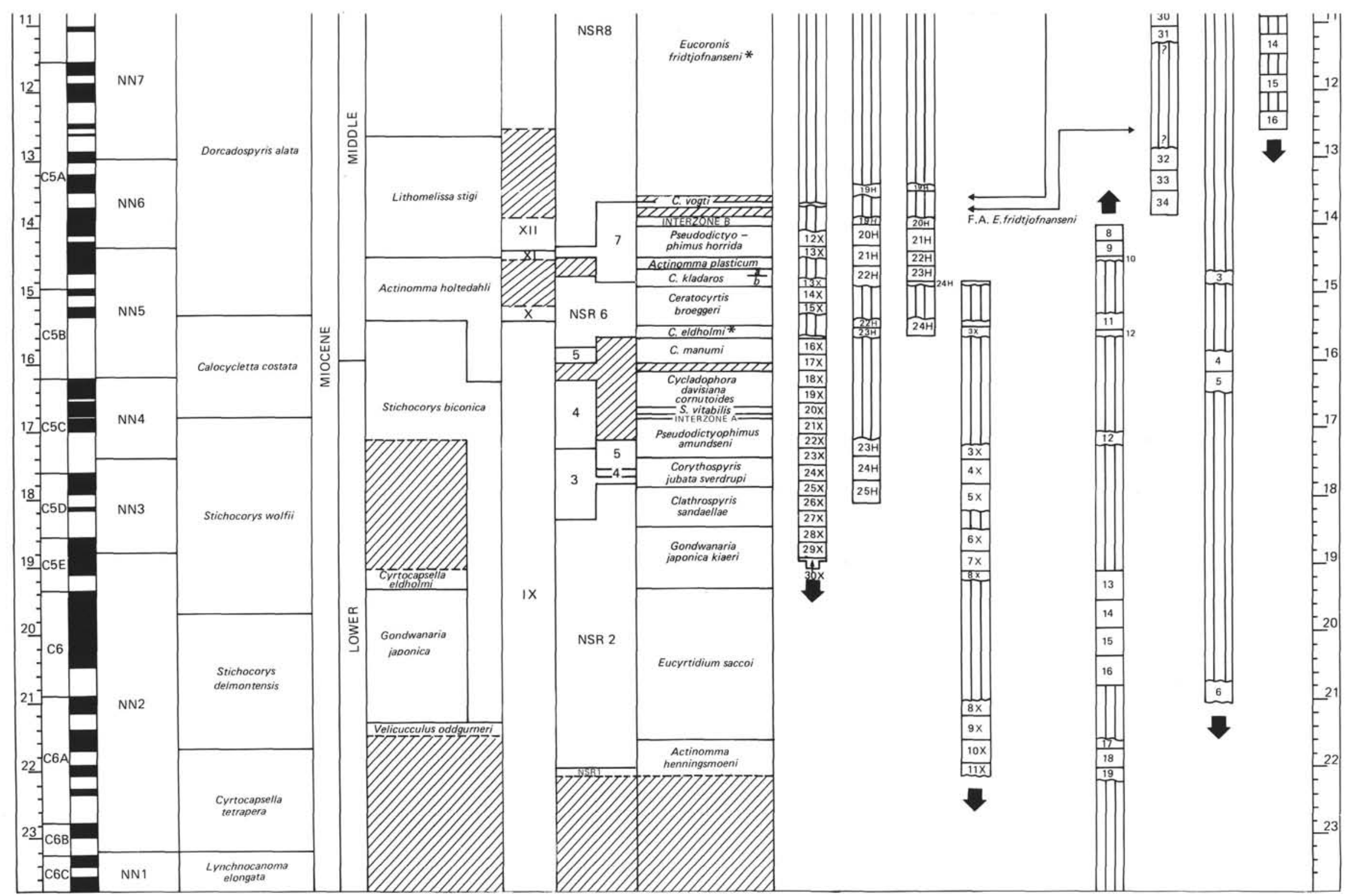

Figure 2. Correlation chart showing the ages of cores containing Neogene biosiliceous sediments recovered on Leg 104 as well as from four holes drilled during Leg 38 . The 28 zones proposed in this paper are compared to previous radiolaria biozonations for the Norwegian Sea and other selected correlation standards. The ages of magnetostratigraphic, calcareous nannoplankton, and series/subseries units follow Berggren et al., 1985. The ages of Leg 104 cores are taken from Goll, (this volume). Ages of the Leg 38 cores are based on the present study, and do not necessarily

follow the determinations of other authors. The broad black arrows indicate that additional non-biosiliceous sediments were recovered above or below the represented interval. Asterisks after zone names indicates zones with mended definitions. F.A. = First Appearance. Vertical lines signify hiatuses or uncored intervals. 
Table 1. Explanation of abundance designations for Tables 2-6.

\begin{tabular}{|c|c|c|c|}
\hline \multicolumn{2}{|c|}{$\begin{array}{l}\text { Criteria for visual estimation } \\
\text { of radiolaria abundance in } \\
\text { our processed residues }\end{array}$} & \multicolumn{2}{|c|}{$\begin{array}{l}\text { Criteria for visual estimation of } \\
\text { the frequency of individual species } \\
\text { in our processed residues }\end{array}$} \\
\hline Designation & $\begin{array}{l}\text { Radiolaria per } \\
\text { field of view }\end{array}$ & Designation & $\begin{array}{c}\text { Specimens per } \\
\text { longitudinal traverse }\end{array}$ \\
\hline B (arren) & 0 & . & 0 \\
\hline $\mathrm{T}$ (race) & $<1$ & $\mathrm{~T}$ (race) & $<1$ \\
\hline$R$ (are) & 1 & $\mathrm{R}$ (are) & 1 \\
\hline$F(e w)$ & $2-4$ & $S$ (parse) & $2-4$ \\
\hline$C$ (ommon) & $5-9$ & C (ommon) & $5-9$ \\
\hline \multirow[t]{5}{*}{ A (bundant) } & $>10$ & A (bundant) & $10-15$ \\
\hline & & V (ery) A (bundant) & $16-20$ \\
\hline & & $\mathrm{D}$ (ominant) & $21-49$ \\
\hline & & V (ery) D (ominant) & $50-100$ \\
\hline & & $F$ (lood) & $>100$ \\
\hline
\end{tabular}

present scheme on us. Neogene radiolarians in Leg 104 recovery appear to fall into two general categories: very long-ranging species having limited biostratigraphic potential and short-ranging species that undergo rapid turnover. To obtain biostratigraphic resolution, we have focused our attention on these latter species, with the consequence that many of our zones have short durations. Nevertheless, 16 of the units in the following scheme are partial-range zones based on first occurrences. For intervals where we have not been able to employ such units, we propose six taxon-range zones, five interval zones, and one concurrent range zone.

Additionally, our scheme includes two provisional interzones: Interzone $\mathrm{A}$ in the upper lower Miocene of Site 643 and Interzone B in the lower middle Miocene of Site 642 (Fig. 2). These short intervals are not treated formally in this section, but they are discussed under BIOSTRATIGRAPHY. The task of more properly characterizing these two units must remain for future, more detailed studies.

The new Neogene radiolarian biozonation for the Norwegian Sea proposed in this paper differs virtually in its entirety from former biozonations for this region proposed by Bjørklund (1976), Dzinoridze et al. (1978), Goll and Bjørklund (1980), and Eldholm, Thiede, Taylor, et al. (1987). Four zonal names proposed by Bjørklund (1976) and Goll and Bjørklund (1980) are retained with emended definitions (Cycladophora davisiana davisiana, Antarctissa whitei, Eucronis fridtjofnanseni, and Cyrtocapsella eldholmi). Six new zones are defined on the basis of previously described species (Pseudodictyophimus gracilipes tetracanthus, Liriospyris cricus, Pseudodictyophimus horrida, Cycladophora davisiana cornutoides, Spongaster tetras, and Clathorospyris sandellae). The remaining 18 new zones and subzones are defined on the basis of new species.

The distribution of radiolarian zones in Leg 104 holes is summarized in Table 7, which includes the boundary criteria as defined below. Table 7 also includes age interpretations for the boundary events, which are based on Figure 2. Ages for the zones and their correlative paleomagnetic chrons are also based on Figure 2. Chronologic ages and paleomagnetic chrons assignments can be made with reasonable confidence for zones younger than 10.2 Ma. These correlations are somewhat interpretive for the older zones (Clathrospyris vogti Zone and below), and the level of confidence declines with increasing age. It is important to note that the chrons indicated in the following definitions are not always consistent with Bleil (this volume). Under the occurrence section of these definitions, we have added information on the distribution of zones in Holes 338, 341, 342, and 348 .

\section{ACTINOMMA HENNINGSMOENI ZONE}

Category: Partial Range Zone

Definition: Interval from the the first occurrence of Actinomma henningsmoeni $\mathrm{n}$. sp. to the first occurrence of Eucyrtidium saccoi.

Status: New zone

Age: Early Miocene; 21.2-21.7 Ma. (interpreted)

Paleomagnetic chron: C6A (interpreted)

Equivalent Zones:

ODP Leg 104 Initial Reports, Zone NSR1 to lower Zone NSR2

DSDP Vol. 38, lower Stichocorys biconia Zone of Site 342, no zonal equivalent at Site 338

Dzinoridze et al. (1978), Zone X

Sakai (1984), upper Cyrtocapsella tetrapera Zone

Martini (1971), Zone NN2

Assemblage: Actinomma henningsmoeni, Actinomma holtedahli (sporadic), Corythospyris fiscella, Corythospyris jubata jubata, Cyrtocapsella tetrapera, Hexalonche ?nakasekoi, Siphocampe anachnea, and Veliccullus oddgurneri.

Occurrence:

Sample 104-642D-10X-1, 25-27 cm to -642D-11X-1, 65-67 cm; 267.4$277.5 \mathrm{mbsf}$

Hole 338, Core 17 Sect. 4, 110-112 cm to Core 19, Sect. 2, 20-25 $\mathrm{cm} ; 167.1-182.2 \mathrm{mbsf}$

\section{EUCYRTIDIUM SACCOI ZONE}

Category: Partial Range Zone

Definition: Interval from the first occurrence of Eucyritidium saccoi to the first occurrence of Gondwanaria japonica kiaeri n. subsp.

Status: New zone

Age: Early Miocene; 20.0/20.5-21.2 Ma (interpreted)

Paleomagnetic chron: C6A (interpreted)

Equivalent Zones:

ODP Leg 104 Initial Reports, mid Zone NSR2

DSDP Vol. 38, Gondwanaria japonica Zone to Velicuccullus oddgurneri Zone of Site 338

Dzinoridze et al., (1978), Zone X

Sakai (1984), Stichocorys delmontensis Zone to lower Stichocorys wolfii Zone

Martini (1971), Zone NN2

Assemblage: Actinomma holtedahli (sporadic), Corythospyris fiscella, Corythospyris jubata jubata, Eucyrtidium saccoi, Cyrtocapsella tetrapera (sporadic), Spongocore puella (sporadic) Hexalonche ?nakasekoi, Siphocampa arachnea, Velicuccullus oddgurneri. Last occurrence of Actinomma henningsmoeni.

Occurrence:

Sample 104-642D-8X-4, 70-72 cm to -642D-9X, CC; $253.0-267.1$ mbsf

Hole 338 , Core 14 , Sect. 1, 55-57 cm to Core 17 , Sect. 3, 35-37 cm; 133.6-164.9 mbsf

Hole 342 , Core 6, Sect. 3, 40-42 cm to Sect. 4, 130-132 cm; $145.4-$ $147.8 \mathrm{mbsf}$

\section{GONDWANARIA JAPONICA KIAERI ZONE}

Category: Partial Range Zone

Definition: Interval from the first occurrence of Gondwanaria japonica kiaeri $\mathrm{n}$. subsp. to the first occurrence of Clathrospyris sandellae. Status: New zone

Remarks: The base of this zone is not recorded in Leg 104 recovery, G. japonica kiaeri must have first occurred on the Vøring Plateau within the time interval represented by a hiatus in Core 104-642D-8 (interpreted interval, 19.3-21.0 Ma).

Age: Early Miocene; 18.5-20.0/20.5 Ma (interpreted)

Paleomagnetic chron: Upper C6 to C5E (interpreted)

Equivalent zones:

ODP Leg 104 Initial Reports, upper Zone NSR2

DSDP Vol. 38, Cyrtocapsella eldholmi Zone

Dzinoridze et al. (1978), Zone X

Sakai (1984), lower Stichocorys wolfi Zone

Martini (1971), Zones NN2-NN3

Assemblage: Velicuccullus oddgurneri, Corythospyris jubata jubata, Corythospyris fiscella, Hexalonche ?nakasekoi, Cyrtocapsella tetrapera 
(sporadic), Siphocampe arachnea (one abundance peak near top), Eucyrtidium saccoi. Last consistent occurrence of Actinomma henningsmoeni in Section 104-643A-29X-2. Last consistent occurrence of $G$. japonica kiaeri at top of zone.

Occurrence:

Sample 104-642D-6X-1, 30-32 cm to -642D-8X-3, 25-27 cm; 228.8$251.1 \mathrm{mbsf}$

Sample 104-643A-28X-1, 105-107 cm to -643A-30X, CC; 255.2 $283.5 \mathrm{mbsf}$

Hole 338, Core 13, Sect. 1, 95-97 cm to Core 13, CC; $124.5-133.0$ mbsf

\section{CLATHROSPYRIS SANDELLAE ZONE}

Category: Partial Range Zone

Definition: Interval from the first occurrence of Clathrospryis sandellae

to the first occurrence of Corythospyris jubata sverdrupi $\mathrm{n}$. subsp.

Status: New zone

Age: Early Miocene; $17.8-18.5 \mathrm{Ma}$ (interpreted)

Paleomagnetic chron: C5D (interpreted)

Equivalent zones:

ODP Leg 104 Initial Reports, lower Zone NSR3 in Hole 643A, upper Zone NSR 2 in Hole 642D

DSDP Vol. 38, no equivalent

Dzinoridze et al. (1978), Zone X at Site 342

Sakai (1984), mid Stichocorys wolfii Zone

Martini (1971), NN3

Assemblage: Velicuccullus oddgurneri, Corythospyris jubata jubata, Corythospyris fiscella, Hexalonche ?nakasekoi (one abundance peak), Cyrtocapsella tetrapera (sporadic traces), Siphocampe arachnea (sporadic), Eucyrtidium saccoi, Eucyrtidium biconicum, Actinomma livae, and Actinomma holtedahli.

Occurrence:

Sections 104-642B-24H, CC to -642B-25H, CC;

Sections 104-642D-4X, CC to -642D-5X, CC; 218.9-228.5 mbsf

Sample 104-643A-25X-4, 105-107 cm to Section 104-643A-27X, CC; 230.3-254.0 mbsf

\section{CORYTHOSPYRIS JUBATA SVERDRUPI ZONE}

Category: Partial Range Zone

Definition: Interval from the first occurrence of Corythospyris jubata sverdrupi $\mathrm{n}$. subsp. to the first occurrence of Pseudodictyophimus amundseni $\mathrm{n} . \mathrm{sp}$.

Status: New zone

Age: Early Miocene; $17.5-17.8 \mathrm{Ma}$ (interpreted)

Paleomagnetic chron: C5C-C5D (interpreted)

Equivalent zones:

ODP Leg 104 Initial Reports mid Zone NSR3 in Hole 643A, and Zone NSR2 to Zone NSR5 in Hole 642D

DSDP Vol. 38, no equivalent

Dzinoridze et al. (1978), Zone X at Site 342

Sakai (1984), upper Stichocorys wolfii Zone

Martini (1971), Zone NN3

Assemblage: Velicuccullus oddgurneri, Corythospyris jubata jubata, Corythospyris fiscella (dominant in Hole 643A), Hexalonche ?nakasekoi (few to dominant), Cyrtocapsella tetrapera (sporadic traces), Eucyrtidium saccoi, Siphocampe arachnea, Clathrospyris sandellae, and Eucyrtidium biconicum (sporadic), Actinomma livae (traces) and Actinomma holtedahli.

Occurrence:

Samples $104-642 \mathrm{~B}-24 \mathrm{H}-1,75-77 \mathrm{~cm}$ to $-642 \mathrm{~B}-24 \mathrm{H}-5,75-77 \mathrm{~cm}$; 206.4-212.4 mbsf

Samples 104-642D-4X-1, 24-26 cm to -642D-4X-6, 24-26 cm; 209.4$217.0 \mathrm{mbsf}$

Samples 104-643A-23X-5, 105-107 cm to -643A-25X-5, 105-107 cm; 212.2-228.8 mbsf

\section{PSEUDODICTYOPHIMUS AMUNDSENI ZONE}

Category: Taxon Range Zone

Definition: Interval including the total consistent range of Pseudodictyophimus amundseni $\mathrm{n}$. $\mathrm{sp}$.

Status: New zone

Index taxon: New species

Remarks: In Leg 104 recovery, occurrences of $P$. amundseni are very sporadic above the top of the zone, and there is a very short interzone in
Core 104-643A-20X, where species diagnostic for neither the $P$. amundseni Zone or Spongotrochus vitabilis Zone are present.

Age: Early Miocene; $16.9-17.5 \mathrm{Ma}$ (interpreted)

Paleomagnetic chron: C5C (interpreted)

Equivalent Zones:

ODP Leg 104 Initial Reports includes the NSR3/NSR4 boundary in Hole 643A, and upper Zone NSR5 of Site 642

DSDP Leg 38, lowermost Stichocorys biconica Zone at Site 338.

Dzinoridze et al. (1978), Zone X at Site 342, Zone XI at Site 338.

Sakai (1984), uppermost Stichocorys wolfii Zone

Martini (1971), NN4

Assemblage: Corythospyris fiscella, Hexalonche ?nakasekoi (three abundance peaks), Clathrospyris sandellae, Siphocampe arachnea (three abundance peaks), Eucyrtidium saccoi and Eucyrtidium biconicum (sporadic), Cyrtocapsella tetrapera, and Actinomma livae (traces). Last consistent occurrences of Velicuccullus oddgurneri, Corythospyris jubata jubata and Corythospyris jubata sverdrupi.

Occurrence:

Sample 104-642B-23H-4, 75-77 cm to Section 104-642D-23X, CC; 201.3-205.6 mbsf

Sample 104-642D-3X-3, 25-27 cm to Section 104-642D-3X, CC; 202.9-207.5 mbsf

Samples $104-643 \mathrm{~A}-21 \mathrm{X}-1,35-37 \mathrm{~cm}$ to $-643 \mathrm{~A}-23 \mathrm{X}-4,105-107 \mathrm{~cm}$; $186.2-210.7 \mathrm{mbsf}$

Hole 338 , Core 12 , Sect. $3,145-147 \mathrm{~cm}$ to Core 12 , CC; $118.5-123.5$ mbsf

\section{SPONGOTROCHUS VITABILIS ZONE}

Category: Partial Range Zone

Definition: Interval from the first occurrence of Spongotrochus vitabilis n. sp. to the first occurrence of Cycladophora davisiana cornutoides.

Status: New zone

Age: Early Miocene; $16.7-16.8 \mathrm{Ma}$ (interpreted)

Paleomagnetic chron: C5C (interpreted)

Equivalent zones:

ODP Leg 104 Initial Reports mid Zone NSR4 in Hole 643A; no equivalent at Site 642

DSDP Vol. 38, Stichocorys biconica Zone

Dzinoridze et al. (1978), Zone X at Site 342, Zone XI at Site 338

Sakai (1984), lowermost Calocycletta costata Zone

Martini (1971), upper Zone NN4

Assemblage: Corythospyris fiscella, Hexalonche ?nakasekoi, Actinomma holtedahli (sporadic), Cyrtocapsella tetrapera (traces), Siphocampe arachnea, Eucyrtidium saccoi, Eucyrtidium biconicum, and Clathrospyris sandellae.

Occurrence:

Samples 104-643A-20X-3, 105-107 cm to -643A-20X-5, 105-107 cm; 180.4-183.4 mbsf

\section{CYCLADOPHORA DAVISIANA CORNUTOIDES ZONE}

Category: Partial Range Zone

Definition: Interval from the first occurrence of Cycladophora davisiana cornutoides to the first occurrence of Ceratocyrtis manumi $\mathrm{n}$. $\mathrm{sp}$. Status: New zone

Age: Early Miocene; 16.0/16.2-16.7 Ma (interpreted)

Paleomagnetic chron: C5C (interpreted)

Equivalent zones:

ODP Leg 104 Initial Reports upper Zone NSR4 in Hole 643A, no equivalents at Site 642 .

DSDP Vol. 38, Stichocorys biconica Zone

Dzinoridze et al. (1978), Zone X at Site 342, Zone XI at Site 338

Sakai (1984), Calocycletta costata Zone

Martini (1971), upper Zone NN4

Assemblage: Hexalonche ?nakasekoi (two abundance peaks), Siphocampe arachnea (three abundance peaks), Actinomma holtedahli and Cyrtocapsella tetrapera (sporadic), Spongocore puella and Acrosphaera spinosa echinoides (traces), Clathrospyris sandellae, Corythospyris hispida and Eucyrtidium biconicum (sporadic), and Eucyrtidium saccoi. Last consistent occurrence of Corythospyris fiscella.

Occurrence:

Sample 104-643A-18X-1, 105-107 cm to $-643 \mathrm{~A}-20 \mathrm{X}-2,105-107 \mathrm{~cm}$; $158.4-178.9 \mathrm{mbsf}$ 
Hole 342 , Core 5, Sect. 2, 55-57 cm to Sect. 6, 110-112 cm; 134.6 $141.1 \mathrm{mbsf}$

\section{CERATOCYRTIS MANUMI ZONE}

Category: Partial Range Zone

Definition: Interval from the first occurrence of Ceratocyrtis manumi n. sp. to the first occurrence of Cyrtocapsella eldholmi n. sp.

Status: New zone

Remarks: This zone is defined as a partial range zone, although $C$. manumi and $C$. eldholmi do not have overlapping ranges in Leg 104 recovery. The top of the zone and the overlying C. eldholmi Zone are missing in Hole 643A, and the base of the C. eldholmi Zone and the $C$. manumi Zone are absent at Site 642 .

Age: Middle Miocene; 15.7-16.0/16.2 Ma

Paleomagnetic chron: $\mathrm{C} 5 \mathrm{~B}$

Equivalent zones:

ODP Leg 104 Initial Reports, includes the NSR5-NSR6 boundary in Hole 643A; no equivalent at Site 642

DSDP Vol. 38, Stichocorys biconica Zone

Dzinoridze et al. (1978), Zone XI at Site 338

Sakai (1984), mid Calocycletta costata Zone

Martini (1971), NN5

Assemblage: Corthospyris fiscella and Hexalonche ?nakasekoi (rare, sporadic), Actinomma holtedahli, Siphocampe arachnea, Eucyritidium saccoi, Corythospyris hispida, Spongocore puella (trace), Clathrospyris sandellae and Actinomma livae (sporadic), Cycladophora davisiana cornutoides. First occurrence of Trisolenia megalactis megalactis in Section 104-643A-16-6.

Occurrence:

Sample 104-643A-15H-5, 105-107 cm to -643A-17X, CC; $135.9-$ 148.5 mbsf

Hole 342, Core 4, Sect. 1, 120-122 cm; 124.2 mbsf

\section{CYRTOCAPSELLA ELDHOLMI ZONE}

Category: Partial Range Zone

Definition: Interval for the first occurrence of Cyrtocapsella eldholmi to the first occurrence of Ceratocyrtis stoermeri. n.sp.

Status: Originally proposed by Bjørklund (1976), emended herein.

Remarks: This zone was originally defined on the basis of multiple boundary criteria. In its broadest sense, the original $C$. eldholmi Zone would probably range from the Gondwanaria japonica kiaeri to the Ceratocyrtis manumi Zones of the present scheme.

Age: Middle Miocene; $15.5-15.7 \mathrm{Ma}$

Paleomagnetic chron: C5B

Equivalent zones:

ODP Leg 104 Initial Reports, Zone NSR6

DSDP Vol. 38, upper Stichocorys biconica Zone

Dzinoridze et al. (1978), Zone XI

Sakai (1984), Calocycletta costata Zone

Martini (1971), NN5

Assemblage: Hexalonche ?nakasekoi, Siphocampe arachnea, Eucyrtidium saccoi, Clathrospyris sandellae, Eucyrtidium biconicum, Cyrtocapsella tetrapera, Cyrtocapsella eldholmi, Actinomma livae, and Corythospyris hispida. Last occurrences of Velicuccullus oddgurneri and Pseudodictyophimus horrida.

Occurrence:

Samples 104-642B-23H-1, 75-77 cm to -642B-23H-3, 75-77 cm; $196.8-199.8 \mathrm{mbsf}$

Sample $104-642 \mathrm{C}-24 \mathrm{H}-5,25-27 \mathrm{~cm}$ to $-642 \mathrm{C}-24 \mathrm{H}, \mathrm{CC} ; 198.7-199.6$ mbsf

Section 104-642D-2X-CC to Sample 104-642D-3X-2, 25-27 cm; 199.7$201.4 \mathrm{mbsf}$

Hole 338, Core 11, Sect. 3, 60-62 cm to Core 12, Sect. 2, 145-147 $\mathrm{cm} ; 108.1-117.0 \mathrm{mbsf}$

\section{CERATOCYRTIS BROEGGERI ZONE}

Category: Interval Zone

Definition: Interval from the first occurrence of Ceratocyrtis stoermeri $\mathrm{n}$. sp. to the first occurrence of Cyrtocapsella kladaros $\mathrm{n}$. $\mathrm{sp}$.

Status: New zone

Remarks: $C$. stoermeri has its advent level one section below that of C. broeggeri in Hole 642B (Table 2, col. 19) and Hole 642C (Table 3, col. 11). The former species is employed as the basal boundary criterion because it is thought to be the precursor of $C$. broeggeri, whose range is more characteristic of the zone. In Leg 104 recovery the ranges of $C$. broeggeri and C. kladaros do not overlap, even at Site 643, where continuous sedimentation is thought to characterize the boundary between the $C$. broeggeri and C. kladaros Zones.

Age: Middle Miocene; $14.9-15.5 \mathrm{Ma}$ (interpreted)

Paleomagnetic chron: C5B (interpreted)

Equivalent zones:

ODP Leg 104, Initial Reports, Zone NSR6

DSDP Vol. 38, upper Stichocorys biconica Zone to lower Actinomma holtedahli Zone

Dzinoridze et al (1978), Zone XI

Sakai (1984), upper Calocycletta costata Zone to lower Dorcadospyris alata Zone

Martini (1971), Zone NN5

Assemblage: Corythospyris fiscella (rare, sporadic), Hexalonche ?nakasekoi (three abundance peaks), Siphocampe arachnea (one abundance peak), Actinomma holtedahli and Cyrtocapsella tetrapera (traces), Eucyrtidium saccoi, Corythospyris hispida, Clathrospyris sandellae, Cycladophora davisiana cornutoides, Acrosphaera spinosa echinoides, and Trisolenia megalactis megalactis (sporadic). First occurrence of Actinomma mirabile.

Occurrence:

Samples 104-642B-22H-6, 75-77 cm to -642B-22H, CC; $194.5-196.0$ mbsf

Samples $104-642 \mathrm{C}-24 \mathrm{H}-3,25-27 \mathrm{~cm}$ to $-642 \mathrm{C}-24 \mathrm{H}-4,25-27 \mathrm{~cm}$; $195.7-197.2 \mathrm{mbsf}$

Sample 104-642D-2X-6, 25-27 cm; $197.7 \mathrm{mbsf}$

Samples $104-643 \mathrm{~A}-14 \mathrm{X}-1,109-111 \mathrm{~cm}$ to $-643 \mathrm{~A}-15 \mathrm{X}-4,25-27 \mathrm{~cm}$; 120.4- $-133.6 \mathrm{mbsf}$

Hole 338 , Core 11 , Sect.1, $55-57 \mathrm{~cm}$ to Sect. $2,55-57 \mathrm{~cm}$; $105.1-$ $106.6 \mathrm{mbsf}$

\section{CYRTOCAPSELLA KLADAROS ZONE (SUBZONE A)}

\section{Cyrtocapsella kladaros Subzone}

Category: Partial Range Zone

Definition: Interval from the first occurrence of Cyrtocapsella kladaros n. sp. to the first occurrence of Cyrtocapsella ampullacea n. sp.

Status: New subzone

Age: Middle Miocene; 14.8-14.9 Ma (interpreted)

Paleomagnetic chron: C5A (interpreted)

Equivalent zones:

ODP Leg 104, Initial Reports, Zone NSR7 in Hole 643A and Zones NSR 3 and NSR6 of Site 642

DSDP Vol. 38, Actinomma holtedahli Zone

Dzinoridze et a1. (1978), Zone XI

Sakai (1984), Dorcadospyris alata Zone

Martini (1971), Zone NN5

Assemblage: Hexalonche ?nakasekoi, Actinomma holtedahli, Siphocampe arachnea, Eucyrtidium saccoi, Corythospyris hispida, Clathrospyris sandellae (sporadic), Actinomma plasticum, and Cyrtocapsella tetrapera (traces).

Occurrence:

Samples $104-642 \mathrm{~B}-22 \mathrm{H}-4,75-77 \mathrm{~cm}$ to $-642 \mathrm{~B}-22 \mathrm{H}-5,75-77 \mathrm{~cm}$; 191.5-193.0 mbsf

Samples $104-642 \mathrm{C}-24 \mathrm{H}-1,25-27 \mathrm{~cm}$ to $-642 \mathrm{C}-24 \mathrm{H}-2,25-27 \mathrm{~cm}$; 192.7-194.2 mbsf

Samples 104-642D-2X-2, 25-27 cm to -642D-2X-4, 25-27 cm; $191.7-$ $194.7 \mathrm{mbsf}$

Sample 104-643A-13H-4, 104-106 cm to Section 104-643A-13H-4, CC; $115.4-119.3 \mathrm{mbsf}$

Hole 338, Core 10, Sect. 2, 60-62 cm; $97.1 \mathrm{mbsf}$

Hole 342, Core 3, Sect. 4, 120-122 cm; 90.7 mbsf

\section{CYRTOCAPSELLA KLADAROS ZONE (SUBZONE B)}

\section{Crytocapsella ampullacea Subzone}

Category: Taxon Range Zone

Definition: Interval including the total range of Cyrtocapsella ampullacea $\mathrm{n} . \mathrm{sp}$.

Status: New subzone

Age: Middle Miocene; 14.7-14.8 Ma (interpreted)

Paleomagnetic chron: C5B (interpreted) 
Equivalent zones:

ODP Leg 104, Initial Reports, Zone NSR7 in Site 642; no equivalent in Hole 643A

DSDP Vol. 38, Actinommia holtedahli Zone

Dzinoridze et al. (1978), lower Zone XI

Sakai (1984), lower Dorcadospyris alata Zone

Martini (1971), Zone NN5

Assemblage: Hexalonche ?nakasekoi, Actinomma holtedahli, Siphocampe arachnea, Clathrospyris sandellae, Eucyrtidium saccoi, Cyrtocapsella tetrapera.

Occurrence:

Sample 104-642B-22H-2, 75-77 cm to -642B-22H-3, 75-77 cm; $188.5-$ $190.0 \mathrm{mbsf}$

Sample 104-642C-23H-3, 24-26 cm to -642C-23H, CC; $187.5-192.4$ mbsf

Sample 104-642D-2X-1, 25-27 cm; $190.2 \mathrm{mbsf}$

\section{ACTINOMMA PLASTICUM ZONE}

Category: Interval Zone

Definition: Interval from the last occurrence of Cyrtocapsella ampullacea $\mathrm{n}$. sp. to the first occurrence of Pseudodictyophimus horrida

Status: New zone

Age: Middle Miocene; 14.5-14.7 Ma (interpreted)

Paleomagnetic chron: C5A (interpreted)

Equivalent zones:

ODP Leg 104, Initial Reports, Zone NSR7 at Site 642; no equivalent in Hole 643A

DSDP Vol. 38, Actinomma holtedahli Zone

Dzinoridze et al. (1978),? Zone XI

Sakai (1984), Dorcadospyris alata Zone

Martini (1971), Zone NN5

Assemblage: Clathrospyris sandellae, Spongocore puella, Hexalonche ?nakasekoi and Actinomma holtedahli (sporadic), Siphocampe arachnea, Eucyrtidium saccoi.

Occurrence:

Samples $104-642 \mathrm{~B}-21 \mathrm{H}-6,24-26 \mathrm{~cm}$ to $-642 \mathrm{~B}-22 \mathrm{H}-1,75-77 \mathrm{~cm}$; 184.8-187.0 mbsf

Samples $104-642 \mathrm{C}-22 \mathrm{H}-4,25-27 \mathrm{~cm}$ to $-642 \mathrm{C}-23 \mathrm{H}-2,24-26 \mathrm{~cm}$; $182.3-186.0 \mathrm{mbsf}$

Hole 338, Core 10, Sect. 1, 115-117 cm; $96.2 \mathrm{mbsf}$

\section{PSEUDODICTYOPHIMUS HORRIDA ZONE}

Category: Taxon Range Zone

Definition: Interval including the total consistent range of Pseudodictyophimus horrida

Status: New zone

Remarks: $P$. horrida has sporadic occurrences in low frequencies above its last consistent range. This overlying interval (Interzone B) is difficult to identify because of the absence of diagnostic species.

Age: Middle Miocene; 14.1-14.5 Ma (interpreted)

Paleomagnetic chron: C5A (interpreted)

Equivalent zones:

ODP Leg 104, Initial Reports, Zone NSR7 at Site 642, includes the NSR7/NSR 8 boundary in Hole 643A

DSDP Vol. 38, Lithomelissa stigi Zone

Dzinoridze et al. (1978), Zones XII-XIII

Sakai (1984), Dorcadospyris alata Zone

Martini (1971), upper Zone NN5 to lower Zone NN6

Assemblage: Hexalonche ?nakasekoi (three abundance peaks), Siphocampe arachnea (one abundance peak), Eucyrtidium saccoi and Cyrtocapsella tetrapera (sporadic), Corythospyris hispida, Clathrospyris sandellae and Actinomma mirabile (sporadic), Artostrobus joergenseni (sporadic). Last occurrences of Acrosphaera spinosa echinoides and Actinomma plasticum.

Occurrence:

Samples 104-642B-19H-6, 24-26 cm to $-642 \mathrm{~B}-21 \mathrm{H}-5,24-26 \mathrm{~cm}$; $167.2-183.3 \mathrm{mbsf}$

Samples $104-642 \mathrm{C}-20 \mathrm{H}-3,25-27 \mathrm{~cm}$ to $-642 \mathrm{C}-22 \mathrm{H}-3,25-27 \mathrm{~cm}$; $161.8-180.8 \mathrm{mbsf}$

Samples 104-643A-12H-3, 105-107 cm to -643A-13H-3, 104-106 cm; 104.4-113.8 mbsf

Hole 338, Core 8, Sect. 2, 53-55 cm to Core 9, CC; 78.0-86.5 mbsf

\section{CLATHROSPYRIS VOGTI ZONE}

Category: Taxon Range Zone

Definition: Interval from the first occurrence of Clathrospyris vogti n. sp. to the first occurrence of Eucoronis fridtjofnanseni.

Status: New zone

Remarks: Neither the top or base of this zone is recorded in Leg 104 recovery, and $C$. vogti and $E$. fridtjofnanseni do not have overlapping stratigraphic ranges in Leg 104 recovery. This zone has been identified only in two core sections described as slump or debris flow deposits, and its age is difficult to interpret. Because $C$. vogti is regarded as the precursor to $E$. fridtjofnanseni, this zone is inferred to be older than the E. fridtjofnanseni Zone, which is not present at Site 643 .

Age: Middle Miocene; 13.5/13.6-13.7/13.8 Ma (interpreted)

Paleomagnetic chron: C5A (interpreted)

Equivalent zones:

ODP Leg 104 Initial Reports, Zone NSR8 in Hole 643A, uppermost Zone NSR7 at Site 642

DSDP Vol. 38, Lithomelissa stigi Zone

Dzinoridze et al. (1978), no equivalent

Sakai (1984), upper Dorcadospyris alata Zone

Martini (1971), NN6

Assemblage: Hexalonche ?nakasekoi (abundant to dominant), Eucyridium saccoi, Clathrospyris sandellae (sporadic traces). Last occurrence of Corythospyris hispida at top of zone.

Occurrence:

Samples 104-643A-12X-1, 105-107 cm to -643A-12X-2, 105-107 cm; 101.4-102.9 mbsf

\section{EUCORONIS FRIDTJOFNANSENI ZONE}

Category: Partial Range Zone

Definition: Interval from the first occurrence of Eucoronis fridtjofnanseni to the first occurrence of Corythospyris reuschi n.sp.

Status: Originally proposed by Goll and Bjørklund (1979), emended herein.

Remarks: This zone was first recognized in the recovery from DSDP Sites 338 and 341 , and the top of the unit was defined as the first occurrence of Antarctissa whitei. As a result of our present study however, it is apparent that there is a large hiatus in Holes 338 and 341 equivalent to the composite section of Leg 104 recovery ranging from the upper $E$. fridjofnanseni Zone to the base of the $A$. whitei Zone.

Age: Middle Miocene to late Miocene; 9.5-13.5/13.6 Ma (interpreted)

Paleomagnetic chron: C5-C5A (interpreted)

Equivalent zones:

ODP Leg 104, Initial Reports, Zone NSR8

DSDP Vol. 38, interzone

Dzinoridze et al., (1978), no equivalent

Sakai (1984), Dorcadospyris alata to Diartus pettersoni Zones

Martini (1971), NN6-NN9

Assemblage: Corythospyris fiscella (sporadic), Hexalonche ?nakasekoi (sporadic, one abundance peak at base of zone), Siphocampe arachnea (sporadic, rare or traces), Clathrospyris sandellae, Botryostrobus auritus (very sporadic). Last occurrence of Actinomma plasticum.

Occurrence:

Samples 104-642B-18H-5, 24-26 cm to -642B-19H-5, 24-26 cm; $154.0-163.9 \mathrm{mbsf}$

Samples $104-642 \mathrm{C}-19 \mathrm{H}-3,25-27 \mathrm{~cm}$ to $-642 \mathrm{C}-20 \mathrm{H}-2,25-27 \mathrm{~cm}$; $152.3-160.3 \mathrm{mbsf}$

Hole 341, Core 27, Sect. 2, 17-19 cm to Core 31, CC; 372.2-425.5 mbsf

\section{EUCORONIS FRIDTJOFNANSENI-CORYTHOSPYRIS REUSCHI ZONE}

Category: Concurrent Range Zone

Definition: Interval from the first occurrence of Corythospyris reuschi

n. sp. to the last occurrence of Eucoronis fridtjofnanseni

Status: New zone

Age: Late Miocene; 8.3/8.6-9.5 Ma

Paleomagnetic chron: $\mathrm{C} 4 \mathrm{~A}-\mathrm{C} 5$

Equivalent zones:

ODP Leg 104 Initial Reports, upper Zone NSR8

DSDP Vol. 38, interzone 


\begin{tabular}{|c|c|c|c|c|c|c|c|c|c|c|c|c|c|c|c|c|c|c|c|c|c|c|c|c|c|c|c|c|c|c|c|c|c|c|c|c|}
\hline $\begin{array}{l}\text { Radiolarian } \\
\text { Zonation }\end{array}$ & Core-Section & $\begin{array}{c}\text { Section } \\
\text { Depth (cm) }\end{array}$ & $\begin{array}{l}\text { Meters below } \\
\text { sea floor }\end{array}$ & $\begin{array}{l}\text { Abundance/ } \\
\text { Preservation }\end{array}$ & & 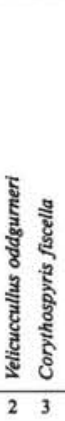 & 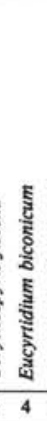 & & 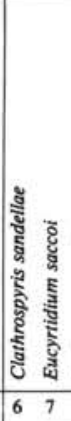 & 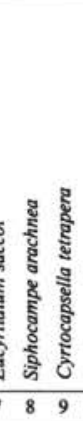 & 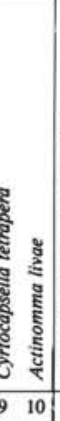 & 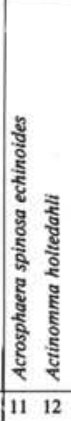 & 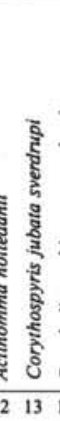 & 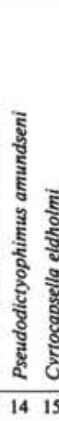 & 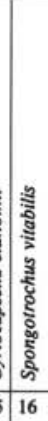 & 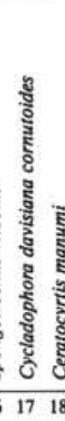 & 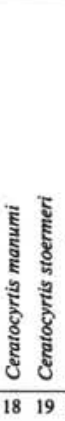 & & 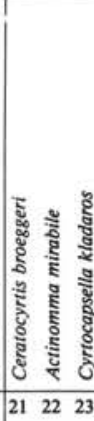 & 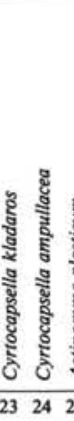 & 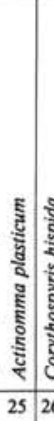 & 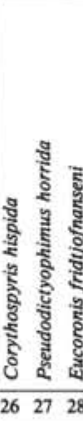 & 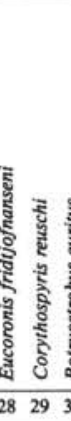 & & 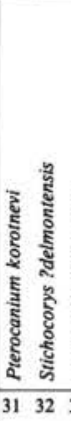 & & 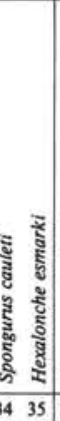 & 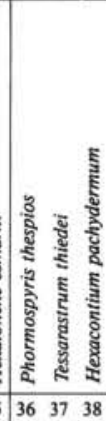 & 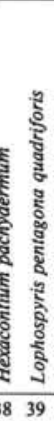 & 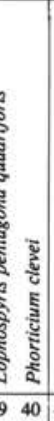 & 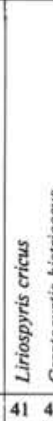 & 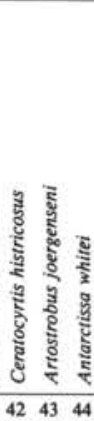 & $\begin{array}{l}\text { हूँ } \\
\text { क्षे } \\
\text { हूँ } \\
\text { हैँ } \\
\text { हैं }\end{array}$ & : & 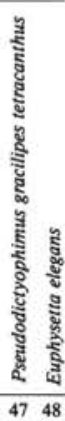 & 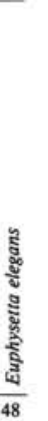 \\
\hline $\begin{array}{l}\text { P. gracilipes } \\
\text { tetracanthus }\end{array}$ & $\begin{array}{l}9 \mathrm{H}-1 \\
9 \mathrm{H}-2 \\
9 \mathrm{H}-3 \\
9 \mathrm{H}-4 \\
9 \mathrm{H}-5 \\
9 \mathrm{H}-6 \\
9 \mathrm{H}-\mathrm{CC} \\
10 \mathrm{H}-1 \\
\end{array}$ & $\begin{array}{l}75-77 \\
75-77 \\
75-77 \\
75-77 \\
75-77 \\
75-77 \\
75-77 \\
\end{array}$ & $\begin{array}{l}67.2 \\
68.7 \\
70.2 \\
71.7 \\
73.2 \\
74.7 \\
75.9 \\
76.7 \\
\end{array}$ & $\begin{array}{l}\text { F/G } \\
\text { F/G } \\
\text { C/G } \\
\text { F/P } \\
\text { F/P } \\
\text { C/M } \\
\text { C/M } \\
\text { F/M }\end{array}$ & $:$ & $\therefore:$ & $\begin{array}{l}\therefore \\
\therefore\end{array}$ & $\vdots$ & $\begin{array}{l}\vdots \\
\vdots \\
\vdots\end{array}$ & $\begin{array}{l}\dot{\mathrm{T}} \\
\dot{\mathrm{T}} \\
\dot{\mathrm{R}} \\
\dot{\mathrm{T}}\end{array}$ & $\begin{array}{l}\vdots \\
\vdots \\
\vdots \\
\vdots \\
\vdots\end{array}$ & $\begin{array}{l}: \\
\vdots \\
\vdots\end{array}$ & $\therefore$ & $\begin{array}{l}: \\
\vdots \\
\vdots\end{array}$ & : & $\begin{array}{l}: \\
\vdots: \\
\vdots:\end{array}$ & $\begin{array}{l}\vdots \\
\vdots \\
\vdots\end{array}$ & $\begin{array}{l}: \\
\vdots \\
\vdots \\
\end{array}$ & $\begin{array}{l}.: \vdots \\
:\end{array}:$ & $: \vdots$ & $\vdots: \vdots$ & $\begin{array}{l}: \\
\vdots \\
\vdots\end{array}$ & $\therefore:$ & \begin{tabular}{l|l}
$\dot{c}$ & $:$ \\
c & $:$ \\
$\mathrm{T}$ & $:$ \\
$\dot{\mathrm{s}}$ & $:$ \\
$\dot{\mathrm{T}}$ & $:$
\end{tabular} & $\begin{array}{l}\vdots \\
\vdots \\
\vdots\end{array}$ & $\begin{array}{l}\vdots \\
\vdots \\
\vdots\end{array}:$ & $\begin{array}{l}\vdots \\
\vdots \\
\vdots\end{array}$ & $: \begin{array}{ll}\vdots \\
\vdots & \vdots \\
\vdots & \mathbf{C} \\
\vdots & \mathbf{R} \\
& \mathbf{R}\end{array}$ & 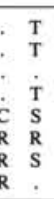 & $\begin{array}{ll}\Gamma & \\
& R \\
R & R \\
S & C \\
R & R \\
S & R \\
& R\end{array}$ & & 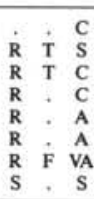 & $\begin{array}{ll}\mathrm{C} & \\
\mathrm{S} & \mathrm{R} \\
\mathrm{C} & \mathrm{R} \\
\mathrm{C} & : \\
\mathrm{A} & : \\
\mathrm{A} & : \\
\mathrm{V} & : \\
\mathrm{S} & :\end{array}$ & : & $\begin{array}{ll}T & \\
R & R \\
R & R \\
R & R \\
T & R \\
T & R \\
C & R \\
S & R \\
S & \text {. }\end{array}$ & $\begin{array}{l}\dot{R} \\
R \\
R \\
R \\
R \\
R \\
R \\
R \\
\end{array}$ \\
\hline A. whitei & $\begin{array}{l}10 \mathrm{H}-2 \\
10 \mathrm{H}-3 \\
10 \mathrm{H}-4 \\
19 \mathrm{H}-5 \\
1 \mathrm{H}-6 \\
1 \mathrm{H}-6 \mathrm{CC} \\
11 \mathrm{H}-1 \\
11 \mathrm{H}-2 \\
11 \mathrm{H}-3 \\
11 \mathrm{H}-4 \\
11 \mathrm{H}-5 \\
11 \mathrm{H}-6\end{array}$ & $\begin{array}{l}25-27 \\
75-77 \\
75-77 \\
25-27 \\
75-77 \\
25-27 \\
25-27 \\
25-27 \\
25-27 \\
25-27 \\
25-27\end{array}$ & $\begin{array}{l}77.7 \\
79.7 \\
81.2 \\
82.2 \\
84.2 \\
85.4 \\
85.7 \\
87.2 \\
88.7 \\
90.2 \\
91.7 \\
93.2\end{array}$ & $\begin{array}{l}\mathrm{C} / \mathrm{M} \\
\mathrm{F} / \mathrm{M} \\
\mathrm{F} / \mathrm{M} \\
\mathrm{C} / \mathrm{M} \\
\mathrm{C} / \mathrm{M} \\
\mathrm{C} / \mathrm{M} \\
\mathrm{A} / \mathrm{M} \\
\mathrm{A} / \mathrm{M} \\
\mathrm{A} / \mathrm{M} \\
\mathrm{C} / \mathrm{P} \\
\mathrm{A} / \mathrm{M} \\
\mathrm{A} / \mathrm{M}\end{array}$ & $\begin{array}{l}\vdots \\
\vdots \\
\vdots \\
\vdots\end{array}$ & $\begin{array}{l}\vdots \vdots \\
\vdots \vdots \\
\vdots \\
\vdots\end{array}$ & $\begin{array}{l}\vdots \\
\vdots \\
\vdots \\
\vdots\end{array}$ & $\begin{array}{l}\vdots \\
\vdots \\
\vdots \\
\vdots\end{array}$ & $\begin{array}{ll} & \mathrm{T} \\
\vdots & \mathrm{T} \\
\vdots & \vdots \\
\vdots & \mathrm{T} \\
\vdots & \mathrm{R} \\
\vdots & \vdots\end{array}$ & $\begin{array}{c}\mathrm{C} \\
\mathrm{A} \\
\vdots \\
\mathrm{D} \\
\mathrm{DD} \\
\mathrm{D} \\
\mathrm{D} \\
\mathrm{R} \\
\mathrm{R} \\
\vdots \\
\vdots\end{array}$ & $\begin{array}{c}\cdot \\
\vdots \\
\vdots \\
\vdots \\
\vdots \\
\vdots\end{array}$ & $\begin{array}{l}\vdots \\
\vdots \\
\vdots \\
\vdots \\
\vdots \\
\vdots\end{array}$ & $\begin{array}{l}\vdots \\
\vdots \\
\vdots \\
\vdots \\
\vdots\end{array}$ & $\begin{array}{l}\vdots \\
\vdots \\
\vdots \\
\vdots \\
\vdots \\
\vdots\end{array}$ & $\begin{array}{l}\vdots \\
\vdots \\
\vdots \\
\vdots\end{array}$ & $\begin{array}{l}: \\
\vdots \\
\vdots \\
\vdots \\
\vdots\end{array}$ & $\begin{array}{l}\vdots \\
\vdots \\
\vdots \\
\vdots \\
\vdots\end{array}$ & \begin{tabular}{l|}
$\vdots$ \\
$\vdots$ \\
$\vdots$ \\
$\vdots$ \\
$\vdots$
\end{tabular} & $\begin{array}{lll}: & \vdots \\
\vdots & \vdots & \vdots \\
\vdots & \vdots & \vdots \\
\vdots & \vdots & \vdots \\
\vdots & \vdots & \vdots \\
\vdots & \vdots & \vdots\end{array}$ & $\begin{array}{l}: \vdots \\
\vdots \vdots \\
\vdots \\
\vdots \\
\vdots\end{array}$ & $\begin{array}{l}\vdots \\
\vdots \\
\vdots \\
\vdots \\
\vdots\end{array}$ & $\begin{array}{l}: \\
\vdots \\
\vdots\end{array}:$ & $\begin{array}{l}\vdots \\
\vdots \\
\vdots \\
\vdots \\
\vdots\end{array}$ & 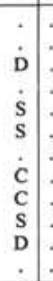 & $\begin{array}{l}\vdots \\
\vdots \\
\vdots \\
\vdots \\
\vdots \\
\vdots \\
\vdots\end{array}$ & $\begin{array}{l}\vdots \\
\vdots \\
\vdots\end{array} \vdots$ & $\begin{array}{l}\vdots \\
\vdots \\
\vdots \\
\vdots \\
\vdots\end{array}$ & 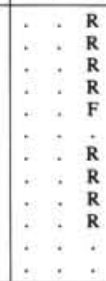 & 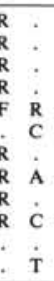 & 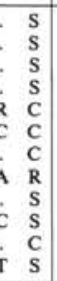 & $\begin{array}{l}: \\
\vdots \\
\vdots \\
\vdots \\
: \\
\vdots \\
\vdots \\
:\end{array}$ & 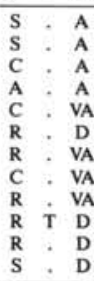 & 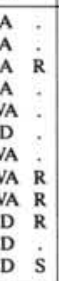 & \begin{tabular}{|l} 
\\
$\vdots$ \\
\\
T \\
R \\
\\
$\vdots$ \\
\\
R
\end{tabular} & $\begin{array}{l}\vdots \\
\vdots \\
\vdots \\
\vdots \\
\vdots\end{array}$ & $\begin{array}{l}\vdots \\
\vdots \\
\vdots\end{array}$ \\
\hline L. cricus & $\begin{array}{l}11 \mathrm{H}-7 \\
11 \mathrm{H}-\mathrm{CC} \\
12 \mathrm{H}-1 \\
12 \mathrm{H}-2 \\
12 \mathrm{H}-3 \\
12 \mathrm{H}-4 \\
12 \mathrm{H}-5 \\
12 \mathrm{H}-6 \\
12 \mathrm{H}-7 \\
12 \mathrm{H}-\mathrm{CC}\end{array}$ & $\begin{array}{l}25-27 \\
25-27 \\
25-27 \\
25-27 \\
25-27 \\
25-27 \\
25-27 \\
25-27\end{array}$ & $\begin{array}{l}94.7 \\
94.9 \\
95.2 \\
96.7 \\
98.2 \\
99.7 \\
101.2 \\
102.7 \\
104.1 \\
104.2 \\
\end{array}$ & $\begin{array}{l}\mathrm{A} / \mathrm{M} \\
\mathrm{A} / \mathrm{M} \\
\mathrm{A} / \mathrm{M} \\
\mathrm{A} / \mathrm{M} \\
\mathrm{A} / \mathrm{M} \\
\mathrm{C} / \mathrm{M} \\
\mathrm{A} / \mathrm{M} \\
\mathrm{A} / \mathrm{M} \\
\mathrm{A} / \mathrm{M} \\
\mathrm{F} / \mathrm{M}\end{array}$ & $\begin{array}{l} \\
\vdots \\
\vdots\end{array}$ & $\begin{array}{l}\vdots \\
\vdots \\
\vdots\end{array}$ & $\begin{array}{l}: \\
\vdots \\
\vdots\end{array}$ & & $\begin{array}{l}\mathrm{R} \\
\vdots \\
\mathrm{T} \\
\mathrm{T} \\
\mathrm{S} \\
\vdots \\
\vdots \\
\vdots\end{array}$ & $\begin{array}{c}\dot{\mathrm{T}} \\
\mathrm{vD} \\
\dot{\mathrm{c}} \\
\dot{\mathrm{s}} \\
\vdots\end{array}$ & $\begin{array}{l}\vdots \\
\vdots \\
\vdots \\
\vdots \\
\vdots\end{array}$ & $\begin{array}{l}\vdots \\
\vdots \vdots \\
\vdots \\
\vdots\end{array}$ & $\begin{array}{l}\vdots \\
\vdots \\
\vdots\end{array}$ & $\begin{array}{l}\vdots \\
\vdots \\
\vdots\end{array} \vdots$ & $\begin{array}{l}\vdots \\
\vdots \\
\vdots\end{array}$ & $\begin{array}{l}\vdots \\
\vdots \\
\vdots\end{array}$ & $\begin{array}{l}\vdots \vdots \\
\vdots \vdots \\
\vdots \\
\vdots\end{array}$ & \begin{tabular}{l|}
$\vdots$ \\
$\vdots$ \\
$\vdots$ \\
$\vdots$
\end{tabular} & $\begin{array}{l}: \\
:\end{array}$ & $\begin{array}{l}5 \\
\vdots \\
\vdots\end{array} \vdots$ & $\begin{array}{ll} & \\
\vdots & \vdots \\
\vdots & \end{array}$ & $\begin{array}{l}\vdots \\
\vdots \vdots \\
\vdots \vdots \\
\vdots\end{array}$ & $\begin{array}{l}\vdots \\
\vdots \\
\vdots\end{array} \vdots$ & 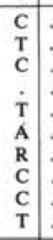 & $\begin{array}{l}\vdots \\
\vdots \\
\vdots \\
\\
\\
\\
\\
\end{array}$ & 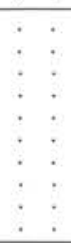 & $\begin{array}{l}\vdots \\
\vdots \\
\vdots\end{array}$ & 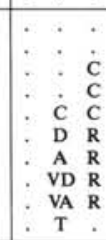 & $\begin{array}{ll} & \vdots \\
\mathrm{C} & \vdots \\
\mathrm{C} & \mathrm{T} \\
\mathrm{C} & \mathrm{T} \\
\mathrm{R} & \mathrm{T} \\
\mathrm{R} & \vdots \\
\mathrm{R} & \vdots \\
\mathrm{R} & \vdots\end{array}$ & $\begin{array}{c}\mathrm{S} \\
\mathrm{R} \\
\mathrm{R} \\
\mathrm{T} \\
\mathrm{T} \\
\mathrm{T} \\
\vdots \\
\vdots \\
\vdots \\
\vdots \\
\mathrm{R} \\
\mathrm{R} \\
\mathrm{T}\end{array}$ & $\begin{array}{ll}\mathrm{R} \\
\mathrm{T} \\
\mathrm{T} \\
\mathrm{T} \\
\mathrm{R} \\
\mathrm{C} \\
\mathrm{T} \\
\mathrm{T} \\
\mathrm{R} \\
\mathrm{R} \\
\mathrm{T} \\
\mathrm{T}\end{array}$ & $\begin{array}{l}\mathrm{C} \\
\mathrm{T}\end{array}$ & $\begin{array}{l}\text { A } \\
\text { A } \\
\text { A } \\
\text { A } \\
\vdots\end{array} \vdots$ & $\begin{array}{l}: \\
: \\
: \\
:\end{array}$ & $\begin{array}{l}\vdots \\
\vdots \\
\vdots\end{array}$ & $\begin{array}{l}5 \\
\vdots \\
\vdots\end{array}$ \\
\hline T. thiedei & $\begin{array}{l}13 \mathrm{H}-1 \\
13 \mathrm{H}-2 \\
13 \mathrm{H}-3 \\
13 \mathrm{H}-4 \\
13 \mathrm{H}-5 \\
13 \mathrm{H}-6 \\
13 \mathrm{H}-7 \\
13 \mathrm{H}-\mathrm{CC} \\
14 \mathrm{H}-1 \\
14 \mathrm{H}-\mathrm{CC} \\
15 \mathrm{H}-1\end{array}$ & $\begin{array}{l}24-26 \\
24-26 \\
24-26 \\
24-26 \\
24-26 \\
24-26 \\
24-26\end{array}$ & $\begin{array}{l}104.4 \\
105.9 \\
107.4 \\
108.9 \\
110.4 \\
111.9 \\
113.4 \\
114.0 \\
114.4 \\
123.5 \\
123.8\end{array}$ & $\begin{array}{l}\mathrm{A} / \mathrm{M} \\
\mathrm{C} / \mathrm{P} \\
\mathrm{A} / \mathrm{P} \\
\mathrm{A} / \mathrm{P} \\
\mathrm{A} / \mathrm{P} \\
\mathrm{A} / \mathrm{P} \\
\mathrm{A} / \mathrm{M} \\
\mathrm{C} / \mathrm{M} \\
\mathrm{C} / \mathrm{M} \\
\mathrm{R} / \mathrm{M} \\
\mathrm{A} / \mathrm{M}\end{array}$ & $\begin{array}{l} \\
\vdots \\
\vdots\end{array}$ & $\begin{array}{l}\vdots: \\
\vdots \vdots \\
\vdots \\
\vdots\end{array}$ & $\begin{array}{l}\vdots \\
\vdots \\
\vdots\end{array}$ & & $\begin{array}{l}{ }^{\mathrm{T}} \\
\vdots \\
\vdots \\
\vdots \\
\vdots \\
\end{array}$ & $\begin{array}{l}\mathrm{VA} \\
\mathrm{R} \\
\dot{\mathrm{S}} \\
\mathrm{S} \\
\mathrm{T} \\
\vdots \\
\vdots \\
\mathrm{R}\end{array}$ & $\begin{array}{l}0 \\
\vdots \\
\vdots \\
\vdots\end{array}$ & 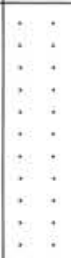 & $\begin{array}{l}\vdots \\
\vdots \\
\vdots \\
\vdots\end{array}$ & $\begin{array}{l}\vdots \\
\vdots \\
\vdots\end{array}:$ & $\vdots \vdots$ & $\begin{array}{l}\vdots: \\
\vdots: \\
\vdots:\end{array}$ & $\begin{array}{l}\vdots \vdots \\
\vdots \vdots \\
\vdots \vdots \\
\vdots\end{array}$ & $\begin{array}{l}\vdots \\
\vdots \\
\vdots \\
\vdots \\
\vdots\end{array}$ & $\begin{array}{lll} & \vdots \\
: & \vdots & \\
\vdots & \vdots & \vdots \\
\vdots & \vdots & \vdots \\
\vdots & \vdots & \vdots\end{array}$ & $\begin{array}{l}\vdots: \\
\vdots \\
\vdots \\
\vdots\end{array}$ & \begin{tabular}{l|l} 
& \\
$\vdots$ & \\
$\vdots$ & \\
$\vdots$ & \\
$\vdots$
\end{tabular} & $\begin{array}{l}\vdots \\
\vdots \vdots \\
\vdots \\
\vdots\end{array}$ & $\begin{array}{l}\vdots \\
\vdots \\
\mathrm{T}\end{array}$ & \begin{tabular}{l|l}
$\mathbf{s}$ & \\
$\mathbf{s}$ & \\
$\dot{c}$ & \\
$\mathbf{c}$ & \\
$\mathbf{T}$ & \\
$\vdots$ & \\
$\vdots$ & \\
$\dot{c}$ &.
\end{tabular} & 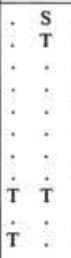 & 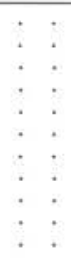 & $\begin{array}{c}\vdots \\
\vdots \\
\vdots \\
\vdots \\
\vdots\end{array}$ & $\mid \begin{array}{lll}\vdots & \text { D } & \text { R } \\
\vdots & \text { D } & \text { R } \\
\vdots & \text { D } & \text { S } \\
\vdots & \text { A } & \text { R } \\
\vdots & \text { D } & \text { C } \\
\vdots & \text { D } \\
\text { T } & \text { D } \\
\text { T } & \text { A } \\
\text { T } & \text { A } \\
\text { T } & \text { D }\end{array}$ & $\begin{array}{ll}S & S \\
\text { R } \\
\text { R } \\
\text { S } \\
\text { R } \\
\text { C } \\
\text { S } \\
\vdots \\
\vdots \\
\vdots \\
\vdots\end{array}$ & $\begin{array}{ll}\mathrm{S} & \mathrm{VA} \\
\vdots & \vdots \\
\mathrm{R} & \vdots \\
\vdots & \vdots \\
\vdots & \vdots \\
\vdots\end{array}$ & $\begin{array}{l}\vdots \\
\vdots \\
\vdots \\
\vdots\end{array}$ & 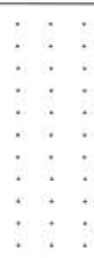 & $\begin{array}{l}\vdots \\
\vdots \\
\vdots \\
\vdots \\
\vdots\end{array}$ & $\begin{array}{l}: \\
\vdots \\
: \\
: \\
:\end{array}$ & $\begin{array}{l}\vdots \\
\vdots \\
\vdots\end{array}$ & $\vdots$ \\
\hline H. esmarki & $15 \mathrm{H}-2$ & $27-29$ & 125.3 & $\mathrm{~A} / \mathrm{M}$ & . & . . & . & &.. & . . . & . . & . . . & . . & . . & . & . . & . . &. & . . . & . . &.. & . . . . &.. & $\mathrm{T} T$ & $\mathrm{~T}$ & . . & . D & .. . . & . & . . & . & .. . . & . . & . & . & \\
\hline S. cauleti & $\begin{array}{l}15 \mathrm{H}-3 \\
15 \mathrm{H}-\mathrm{CC} \\
16 \mathrm{H}-1 \\
16 \mathrm{H}-2\end{array}$ & $\begin{array}{l}27-29 \\
31-33 \\
31-33\end{array}$ & $\begin{array}{l}126.8 \\
128.0 \\
128.4 \\
129.9\end{array}$ & $\begin{array}{l}\mathrm{F} / \mathrm{P} \\
\mathrm{C} / \mathrm{P} \\
\mathrm{C} / \mathrm{M} \\
\mathrm{A} / \mathrm{M}\end{array}$ & : & $\therefore:$ & $:$ & & $\vdots \quad \vdots$ & $\begin{array}{l}T \\
\mathrm{D} \\
\mathrm{D} \\
\mathrm{D} \\
\mathrm{VA} \\
\mathrm{VA}\end{array}$ & $\begin{array}{l}\vdots \\
\mathrm{T}\end{array}$ & & $\therefore$ & $\therefore:$ & $\vdots$ & $\vdots:$ & $\therefore:$ & $\vdots$ & 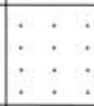 & $\vdots:$ & $\vdots$ & $\begin{array}{l}\vdots \\
\vdots \quad\end{array}$ & $\therefore \begin{array}{l}\mathrm{T} \\
\mathrm{T} \\
\mathrm{T}\end{array}$ & $\begin{array}{l}\mathrm{T} \\
\vdots \\
\mathrm{D}\end{array}$ & $\begin{array}{l} \\
\dot{\mathrm{T}}\end{array}$ & $\begin{array}{ll} & \mathrm{T} \\
\therefore & \mathrm{C} \\
\mathrm{D} & \mathrm{A}\end{array}$ & $\begin{array}{ll}\mathrm{T} & : \\
\mathrm{C} & : \\
\mathrm{A} & : \\
\mathrm{D} & :\end{array}$ & $\vdots: \vdots$ & $\vdots:$ & $\vdots:$ & : & $\begin{array}{l}: \vdots: \\
\vdots:\end{array}$ & $\therefore$ & & $::$ & \\
\hline C. reuschi & $\begin{array}{l}16 \mathrm{H}-3 \\
16 \mathrm{H}-4 \\
16 \mathrm{H}-5 \\
16 \mathrm{H}-6 \\
16 \mathrm{H}-7\end{array}$ & $\begin{array}{l}31-33 \\
31-33 \\
31-33 \\
31-33 \\
31-33\end{array}$ & $\begin{array}{l}131.4 \\
132.9 \\
134.4 \\
135.9 \\
137.4\end{array}$ & $\begin{array}{l}\mathrm{C} / \mathrm{M} \\
\mathrm{A} / \mathrm{M} \\
\mathrm{A} / \mathrm{M} \\
\mathrm{A} / \mathrm{M} \\
\mathrm{A} / \mathrm{M}\end{array}$ & : & $\therefore:$ & $\therefore$ & & $\begin{array}{l}\vdots \\
\vdots \\
\mathrm{R}\end{array}$ & $\begin{array}{l}\text { A } \\
\text { s } \\
\text { c } \\
\text { c }\end{array}$ & $\vdots \vdots$ & $\vdots \vdots$ & : & $\therefore:$ & : & $\vdots \vdots$ & $\vdots \vdots$ & $:$ & $: \vdots:$ & $\therefore:$ & $\vdots$ & $\begin{array}{l}\vdots \\
\vdots\end{array}$ & $\begin{array}{l}\mathrm{T} \\
\therefore \mathrm{T} \\
\mathrm{D} \\
\mathrm{VA} \\
\mathrm{D}\end{array}$ & \begin{tabular}{l|l}
$c$ & \\
$c$ & \\
$c$ & \\
$c$ & \\
$s$ & 1
\end{tabular} & $\begin{array}{|ll|}A & : \\
R & \vdots \\
S & \dot{R} \\
T & : \\
T & \vdots \\
T\end{array}$ & $\vdots:$ & $\vdots:$ & $\mid \vdots \vdots \vdots$ & $\vdots \vdots$ & $\vdots \vdots$ & : & $\begin{array}{l}\vdots \\
\vdots\end{array}:$ & $\vdots:$ & : & $: \vdots$ & $\bar{\vdots}$ \\
\hline
\end{tabular}




\begin{tabular}{|c|c|c|c|c|c|c|c|c|c|c|c|c|c|c|c|c|c|c|c|c|c|c|c|c|c|c|c|c|c|c|c|c|c|c|c|c|c|c|c|c|c|c|}
\hline $\begin{array}{l}\text { E. fridtjofnanseni/ } \\
\text { c. Reuschi }\end{array}$ & $\begin{array}{l}16 \mathrm{H}-\mathrm{CC} \\
17 \mathrm{H}-1 \\
17 \mathrm{H}-2 \\
17 \mathrm{H}-3 \\
17 \mathrm{H}-4 \\
17 \mathrm{H}-5 \\
17 \mathrm{H}-6 \\
17 \mathrm{H}-7 \\
17 \mathrm{H}-\mathrm{CC} \\
18 \mathrm{H}-1 \\
18 \mathrm{H}-2 \\
18 \mathrm{H}-4 \\
\end{array}$ & $\begin{array}{l}31-33 \\
31-33 \\
31-33 \\
31-33 \\
31-33 \\
31-33 \\
31-33 \\
24-26 \\
24-26 \\
24-26 \\
\end{array}$ & $\begin{array}{l}138.0 \\
138.3 \\
139.8 \\
141.3 \\
142.8 \\
144.3 \\
145.8 \\
147.3 \\
147.7 \\
148.0 \\
149.5 \\
152.5 \\
\end{array}$ & $\begin{array}{l}\text { C/M } \\
\text { A/G } \\
\text { F/G } \\
\text { F/G } \\
\text { C/G } \\
\text { C/G } \\
\text { C/G } \\
\text { C/G } \\
\text { F/G } \\
\text { C/G } \\
\text { C/G } \\
\text { C/G } \\
\end{array}$ & $\begin{array}{l}\vdots \\
\vdots \\
\vdots \\
\vdots \\
\vdots \\
\vdots\end{array}$ & $\begin{array}{l}\vdots \\
\vdots \\
\vdots \\
\vdots \\
\vdots \\
\vdots \\
\vdots\end{array}$ & $\begin{array}{l}\vdots \\
\vdots \\
\vdots \\
\vdots \\
\vdots\end{array}$ & $\begin{array}{l}\vdots \\
\vdots \\
\vdots \\
\vdots \\
\mathrm{A} \\
\mathrm{C} \\
\mathrm{C} \\
\mathrm{R}\end{array}$ & 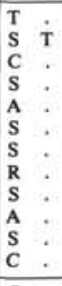 & $\begin{array}{l}\mathrm{T} \\
\vdots \\
\mathrm{c} \\
\vdots \\
\mathrm{T} \\
\mathrm{T} \\
\mathrm{T} \\
\mathrm{T} \\
\mathrm{T}\end{array}$ & $\begin{array}{l}\vdots \\
\vdots \\
\vdots \\
\vdots\end{array}$ & & $\begin{array}{l}\vdots \\
\vdots \\
\vdots \\
\vdots \\
\vdots \\
\vdots \\
\vdots \\
\end{array}$ & $\begin{array}{l}\vdots \\
\vdots \\
\mathrm{T}\end{array}$ & $\begin{array}{l}\vdots \\
\vdots \\
\vdots \\
\vdots\end{array}$ & $:$ & $\begin{array}{l}\vdots \\
\vdots \\
\vdots\end{array}$ & $\begin{array}{l}\vdots \\
\vdots \\
\vdots\end{array}$ & $\begin{array}{l}\vdots \vdots \\
\vdots \\
\vdots \\
\vdots\end{array}$ & $\begin{array}{l}\vdots \\
\vdots \\
\vdots \\
\vdots \\
\end{array}$ & . & $\begin{array}{l}\vdots: \\
\vdots: \\
\vdots \\
\vdots\end{array}$ & $\begin{array}{l}\vdots: \\
\vdots \\
\vdots\end{array}:$ & $\begin{array}{l}\vdots \vdots \\
\vdots \\
\vdots \\
\vdots\end{array}$ & $\begin{array}{l}\vdots \\
\vdots \\
\vdots \\
\vdots \\
\vdots\end{array}$ & $\begin{array}{c}\vdots \\
\mathrm{T} \\
\vdots \\
\vdots \\
\vdots \\
\vdots\end{array}$ & $\begin{array}{lll}\text { T } & \\
\text { S } & \\
\text { C } & \\
\text { S } & \\
\text { A } & \\
\text { S } & \\
\text { S } & 1 \\
\text { R } & \\
\text { S } & \\
\text { A } & \\
\text { S } & \text { S } \\
\text { A } & \\
\text { A } & & \\
\end{array}$ & 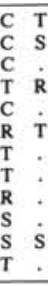 & : & $\begin{array}{l}\vdots \\
\vdots \\
\vdots\end{array}$ & $\begin{array}{l}\vdots \\
\vdots \\
\vdots \\
\vdots \\
\vdots \\
\vdots\end{array}$ & $\begin{array}{l}\vdots \\
\vdots \\
\vdots \\
\vdots\end{array}$ & $\frac{\vdots}{\vdots}:$ & $\begin{array}{l}\vdots \\
\vdots \\
\vdots \\
\vdots \\
\vdots\end{array}$ & $\begin{array}{l}\vdots \\
\vdots \\
\vdots\end{array}$ & $\begin{array}{l}\vdots \\
\vdots \\
\vdots\end{array}$ & $\begin{array}{l}\vdots \\
\vdots \\
\vdots\end{array}$ & $\vdots$ & $\begin{array}{l}\vdots \\
\vdots \\
\vdots \\
\vdots \\
\vdots\end{array}$ & $\begin{array}{l}\vdots: \\
\vdots \vdots \\
\vdots\end{array}$ & $:$ & $\begin{array}{l}\vdots \\
\vdots \\
\vdots \\
\vdots \\
\vdots\end{array}$ \\
\hline E. fridtjofnanseni & $\begin{array}{l}18 \mathrm{H}-5 \\
18 \mathrm{H}-6 \\
18 \mathrm{H}-\mathrm{CC} \\
19 \mathrm{H}-1 \\
19 \mathrm{H}-2 \\
19 \mathrm{H}-3 \\
19 \mathrm{H}-4 \\
19 \mathrm{H}-5\end{array}$ & $\begin{array}{l}24-26 \\
24-26 \\
24-26 \\
24-26 \\
24-26 \\
24-26 \\
24-26\end{array}$ & $\begin{array}{l}154.0 \\
155.0 \\
157.4 \\
157.9 \\
159.4 \\
160.9 \\
162.4 \\
163.9\end{array}$ & $\begin{array}{l}\text { C/G } \\
\text { F/G } \\
\text { R/G } \\
\text { F/G } \\
\text { F/G } \\
\text { C/G } \\
\text { C/G } \\
\text { A/M }\end{array}$ & $\vdots: \vdots$ & $\therefore:$ & $:$ & \begin{tabular}{l|l}
$\mathrm{C}$ & $\mathrm{C}$ \\
$\mathrm{R}$ & \\
$\dot{S}$ & \\
$\dot{c}$ & \\
$\mathrm{~F}$ &
\end{tabular} & $\begin{array}{l}\mathrm{C} \\
\mathrm{C} \\
\mathrm{C} \\
\mathrm{C} \\
\mathrm{S} \\
\mathrm{R} \\
\mathrm{S} \\
\mathrm{S} \\
\mathrm{A}\end{array}$ & $\begin{array}{l}\vdots \\
\vdots \\
\text { s }\end{array}$ & $\begin{array}{l}\vdots \\
\vdots \\
\vdots\end{array}$ & $:$ & $\begin{array}{l}:{ }^{\mathrm{T}} \\
\vdots \\
\vdots\end{array}$ & : : & $:$ & $:$ & : & $\vdots:$ & $\begin{array}{l}\vdots \\
\vdots \\
\vdots\end{array}$ & & $:$ & & 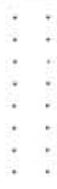 & $: \vdots:$ & : & $\begin{array}{l}\vdots \\
\vdots \\
\vdots\end{array}$ & $\begin{array}{l}\text { A } \\
\text { C } \\
\text { C } \\
\text { A } \\
\text { S } \\
\text { C } \\
\text { R } \\
\text { A }\end{array}$ & $::$ & : & : & $\begin{array}{l}\vdots \\
\vdots \\
\vdots\end{array}$ & $\begin{array}{l}\vdots: \\
\vdots: \\
\vdots \\
\vdots\end{array}$ & $:$ & $\begin{array}{l}\vdots \\
\vdots \\
\vdots\end{array}$ & $\begin{array}{l}\vdots \\
\vdots\end{array}$ & $:$ & : & $\begin{array}{l}\vdots \\
\vdots\end{array}$ & $\begin{array}{l}\vdots \\
\vdots \\
\vdots\end{array}$ & $\begin{array}{l}\vdots: \\
\vdots\end{array}$ & : & : \\
\hline Interzone B & $\begin{array}{l}19 \mathrm{H}-6 \\
19 \mathrm{H}-7 \\
19 \mathrm{H}-\mathrm{CC}\end{array}$ & $\begin{array}{l}24-26 \\
24-26\end{array}$ & $\begin{array}{l}165.4 \\
166.9 \\
167.2 \\
\end{array}$ & $\begin{array}{l}\mathrm{A} / \mathrm{M} \\
\mathrm{A} / \mathrm{G} \\
\mathrm{C} / \mathrm{M}\end{array}$ & $\vdots:$ & $\therefore:$ & & $\begin{array}{l}\mathrm{F} \\
\mathrm{A} \\
\mathrm{D}\end{array}$ & $\begin{array}{l}c \\
\text { c } \\
\text { s }\end{array}$ & $\dot{\mathrm{T}}$ & $\vdots$ & $\vdots$ & $\therefore$ & 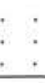 & : & $\therefore$ & : & $\vdots:$ & $\therefore:$ & : & : & $\vdots:$ & $\therefore:$ & $\therefore$ & & $\begin{array}{l}\mathrm{T} \\
\vdots \\
\end{array}$ & $\therefore:$ & $\therefore:$ & : & $\vdots$ & $\vdots:$ & $\therefore:$ & : & $\vdots$ & $\therefore:$ & : : & : & $\vdots$ & $\vdots$ & $\therefore:$ & : & $\vdots:$ \\
\hline P. horrida & $\begin{array}{l}20 \mathrm{H}-1 \\
2 \mathrm{H}-2 \\
20 \mathrm{H}-3 \\
20 \mathrm{H}-4 \\
20 \mathrm{H}-5 \\
20 \mathrm{H}-6 \\
20 \mathrm{H}-7 \\
20 \mathrm{H}-\mathrm{CC} \\
21 \mathrm{H}-1 \\
21 \mathrm{H}-2 \\
21 \mathrm{H}-3 \\
21 \mathrm{H}-4 \\
21 \mathrm{H}-5 \\
\end{array}$ & $\begin{array}{l}24-26 \\
24-26 \\
24-26 \\
24-26 \\
24-26 \\
24-26 \\
24-26\end{array}$ & $\begin{array}{l}167.6 \\
169.1 \\
170.6 \\
172.1 \\
173.6 \\
175.1 \\
176.6 \\
177.1 \\
177.3 \\
178.8 \\
180.3 \\
181.8 \\
183.3 \\
\end{array}$ & $\begin{array}{l}\text { A/G } \\
\text { A/G } \\
\text { F/G } \\
\text { F/G } \\
\text { F/G } \\
\text { C/G } \\
\text { A/G } \\
\text { A/G } \\
\text { A/G } \\
\text { C/G } \\
\text { A/G } \\
\text { A/G } \\
\text { F/G } \\
\end{array}$ & $\begin{array}{l}\vdots \\
\vdots \\
\vdots\end{array}$ & $\begin{array}{l}\vdots \\
\vdots \\
\vdots\end{array} \vdots$ & $\begin{array}{l}\vdots \\
\vdots \\
\vdots \\
\vdots v \\
\vdots V \\
\vdots\end{array}$ & 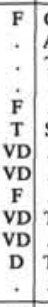 & 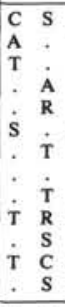 & $\begin{array}{l}\dot{\mathrm{T}} \\
\vdots \\
\dot{\mathrm{c}}\end{array}$ & $\begin{array}{l} \\
\vdots \\
\text { T } \\
\dot{s} \\
\vdots \\
\text { s }\end{array}$ & $\begin{array}{l}\vdots \\
\vdots \\
\vdots \\
\vdots \\
\mathrm{r}\end{array}$ & & $\begin{array}{l}0: \\
\vdots \\
\vdots\end{array}$ & $\begin{array}{l}\vdots \\
\vdots \\
\vdots \\
\vdots\end{array}$ & & $\begin{array}{l}: \\
\vdots \\
\vdots \\
\vdots \\
\text { s }\end{array}$ & $\begin{array}{l}: \\
\vdots \\
\vdots\end{array}$ & $\begin{array}{l}\vdots \\
\vdots \\
\vdots \\
\vdots\end{array}$ & & : & $\begin{array}{l} \\
\vdots \\
\vdots \\
\vdots \\
\text { A } \\
\text { R } \\
S \\
S \\
\text { S } \\
\text { T } \\
\text { T } \\
\end{array}$ & $\begin{array}{l}\vdots \vdots \\
\vdots \\
\vdots\end{array}$ & $\begin{array}{l}\vdots \\
\vdots \vdots \\
\vdots \\
\vdots \\
\vdots \\
\mathrm{S} \\
\mathrm{S} \\
\mathrm{S} \\
\mathrm{S} \\
\vdots \\
\mathrm{S} \\
\mathrm{S} \\
\mathrm{T} \\
\end{array}$ & \begin{tabular}{|l}
$\mathrm{S}$ \\
$\dot{\mathrm{T}}$ \\
$\mathrm{C}$ \\
$\mathrm{S}$ \\
$\mathrm{S}$ \\
$\mathrm{R}$ \\
$\mathrm{A}$ \\
$\mathrm{A}$ \\
$\mathrm{R}$ \\
$\mathrm{S}$ \\
$\mathrm{S}$ \\
\end{tabular} & $\begin{array}{l}\mathrm{S} \\
\mathrm{T} \\
\mathrm{T} \\
\mathrm{S} \\
\mathrm{T} \\
\mathrm{T} \\
\mathrm{S} \\
\mathrm{R} \\
\mathrm{R} \\
\mathrm{S} \\
\mathrm{T} \\
\mathrm{T} \\
\mathrm{T} \\
\end{array}$ & $\begin{array}{l}\vdots: \\
\vdots: \\
\vdots: \\
\vdots\end{array}$ & $:$ & $:$ & $\begin{array}{l}: \\
\vdots \\
\vdots \\
\vdots\end{array}$ & $\begin{array}{l}0: \\
\vdots \vdots \\
\vdots \\
\vdots \\
\vdots \\
\vdots \\
\vdots\end{array}$ & $\begin{array}{l}\vdots: \\
\vdots \vdots \\
\vdots \\
\vdots\end{array}$ & $\begin{array}{l}\vdots \\
\vdots \\
\vdots \\
\vdots\end{array}$ & $\begin{array}{l} \\
\vdots \\
\vdots \\
\vdots \\
\vdots \\
\vdots\end{array}$ & $\begin{array}{l}0: \\
\vdots: \\
\vdots \\
\vdots\end{array}$ & $\therefore:$ & $\begin{array}{l}: \\
: \\
:\end{array}$ & $\begin{array}{l}\vdots \\
\vdots \\
\vdots \\
\vdots\end{array}$ & $\begin{array}{l}0 \\
\vdots \\
\vdots \\
\vdots \\
\vdots \\
\end{array}$ & $\begin{array}{l}\therefore: \\
\vdots \\
\vdots \\
\vdots\end{array}$ & $\begin{array}{l}: \\
: \\
:\end{array}$ & $\begin{array}{l}0: \\
\vdots: \\
\vdots \\
\vdots \\
\vdots\end{array}$ \\
\hline A. plasticum & $\begin{array}{l}21 \mathrm{H}-6 \\
21 \mathrm{H}-7 \\
21 \mathrm{H}-\mathrm{CC} \\
22 \mathrm{H}-1 \\
\end{array}$ & $\begin{array}{l}24-26 \\
24-26\end{array}$ & $\begin{array}{l}184,8 \\
186.3 \\
186.6 \\
187.0 \\
\end{array}$ & $\begin{array}{l}\text { F/G } \\
\text { F/G } \\
\text { F/G } \\
\text { A/G }\end{array}$ & $\therefore:$ & $\therefore:$ & $\begin{array}{l}\mathrm{R} \\
\mathrm{T} \\
\mathrm{T} \\
\mathrm{R} \\
\end{array}$ & 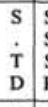 & $\begin{array}{ll}\mathrm{S} & \mathrm{T} \\
\mathrm{S} & \mathrm{T} \\
\mathrm{S} & \mathrm{S} \\
\mathrm{R} & \mathrm{R} \\
\end{array}$ & $\vdots$ & $\vdots$ & & $\begin{array}{l}\text { R } \\
\vdots: \text { T } \\
\vdots \\
\end{array}$ & $\begin{array}{l}\text { i : } \\
\text { s: }\end{array}$ & : & & : & $\therefore:$ & $\vdots:$ & & : & $\therefore:$ & $\therefore:$ & $\begin{array}{l}\mathrm{R} \\
\mathrm{T} \\
\mathrm{T} \\
\mathrm{R}\end{array}$ & \begin{tabular}{|l|}
$\mathbf{T}$ \\
$\mathbf{R}$ \\
$\mathrm{T}$ \\
\\
\end{tabular} & $\vdots$ & $\vdots:$ & $: \vdots$ & $\vdots$ & : & $\therefore:$ & $\therefore:$ & $\therefore$ & $\vdots:$ & $\therefore:$ & $: \vdots$ & : & $\therefore$ & $:$ & $\therefore:$ & : & $: \vdots$ \\
\hline C. kladaras $\frac{b}{a}$ & $\begin{array}{l}22 \mathrm{H}-2 \\
22 \mathrm{H}-3 \\
22 \mathrm{H}-4 \\
22 \mathrm{H}-5\end{array}$ & $\begin{array}{l}75-77 \\
75-77 \\
75-77 \\
75-77\end{array}$ & $\begin{array}{l}188.5 \\
190.0 \\
191.5 \\
193.0\end{array}$ & $\begin{array}{l}\text { A/G } \\
\text { C/G } \\
\text { A/G } \\
\text { A/G }\end{array}$ & & $\therefore:$ & $\begin{array}{l}\dot{v} \\
\dot{T} \\
T\end{array}$ & & $\begin{array}{ll}\mathrm{T} & \mathrm{S} \\
\mathrm{C} & \mathrm{C} \\
\mathrm{S} & \mathrm{R} \\
\mathrm{T} & \mathrm{T}\end{array}$ & $\begin{array}{l}\text { D } \\
\text { A }\end{array}$ & $\begin{array}{l}\mathrm{T} \\
\mathrm{T} \\
\mathrm{T} \\
\mathrm{T}\end{array}$ & & $\begin{array}{l}\mathrm{C} \\
\vdots \\
\mathrm{T} \\
\mathrm{A}\end{array}$ & $\begin{array}{l}c: \\
\text { T: } \\
\text { A : }\end{array}$ & $\vdots$ & & : & $: \vdots$ & $\vdots:$ & & : & $\begin{array}{ll}\text { S } & \text { R } \\
\text { R } & \text { T } \\
\text { T } & \text { S } \\
\text { S } & \text { R }\end{array}$ & $\begin{array}{ll}\dot{\mathrm{T}} & \mathrm{T} \\
\mathrm{S} & \mathrm{s} \\
\mathrm{R} & \end{array}$ & s: & & $\vdots$ & $:$ & $:$ & : & $\vdots$ & $\vdots:$ & $\therefore:$ & : & $\vdots:$ & $::$ & $::$ & : & $\vdots$ & $\vdots$ & $\vdots:$ & : & $::$ \\
\hline C. broeggeri & $\begin{array}{l}22 \mathrm{H}-6 \\
22 \mathrm{H}-\mathrm{CC}\end{array}$ & $75-77$ & $\begin{array}{l}194.5 \\
196.0\end{array}$ & $\begin{array}{l}\mathrm{C} / \mathrm{G} \\
\mathrm{C} / \mathrm{M}\end{array}$ & & $\therefore:$ & T & & $\begin{array}{ll}\mathbf{S} & \mathbf{S} \\
\text {. } & \mathbf{R} \\
\end{array}$ & $\begin{array}{c}\mathrm{T} \\
\mathrm{VA} \\
\end{array}$ & & & $\begin{array}{l}\mathrm{T} \text { VA } \\
. \mathrm{S} \\
\end{array}$ & IA : & : & & : & & $\dot{\mathrm{T}} \dot{\mathrm{s}}$ & $\dot{\mathrm{T}}$ & s & R. & : : & $\therefore:$ & : & $:$ & $:$ & : : & & . & $\therefore:$ & $\therefore:$ & : & $:$ & $\therefore:$ & : : & : & : & $:$ & $\therefore:$ & : & $:$ : \\
\hline C. eldholmi & $\begin{array}{l}23 \mathrm{H}-1 \\
23 \mathrm{H}-2 \\
23 \mathrm{H}-3\end{array}$ & $\begin{array}{l}75-77 \\
75-77 \\
75-77\end{array}$ & $\begin{array}{l}196.8 \\
198.3 \\
199.8\end{array}$ & $\begin{array}{l}\text { C/G } \\
\text { C/G } \\
\text { F/G }\end{array}$ & & $\begin{array}{r}\mathbf{R} \\
\vdots \quad \\
\mathbf{R}\end{array}$ & $\begin{array}{l}\mathrm{T} \\
\mathrm{T} \\
\mathrm{T}\end{array}$ & 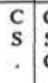 & 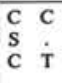 & $\begin{array}{l}\dot{R} \\
\mathrm{R}\end{array}$ & & & $\vdots:$ & $\therefore$ & T & & $\begin{array}{l}\text { R } \\
:\end{array}$ & & $\therefore$ & & $\vdots$ & $\therefore:$ & $\therefore:$ & $\therefore$ & $\vdots$ & $\vdots$ & & & & $\vdots$ & $\therefore:$ & $\therefore:$ & & $\therefore$ & $\therefore:$ & $\therefore$ & & : & $\vdots$ & $\therefore:$ & & $\therefore:$ \\
\hline P. amundseni & $\begin{array}{l}23 \mathrm{H}-4 \\
23 \mathrm{H}-5 \\
23 \mathrm{H}-6 \\
23 \mathrm{H}-\mathrm{CC}\end{array}$ & $\begin{array}{l}75-77 \\
75-77 \\
75-77\end{array}$ & $\begin{array}{l}201.3 \\
202.8 \\
204.3 \\
205.6 \\
\end{array}$ & $\begin{array}{l}\text { C/G } \\
\text { A/G } \\
\text { C/G } \\
\text { A/G } \\
\end{array}$ & & $\begin{array}{ll}\mathrm{R} & \dot{\mathrm{A}} \\
\mathrm{A} & \mathrm{S} \\
\mathrm{A} & \dot{\mathrm{R}} \\
\mathrm{A} & \dot{\mathrm{R}} \\
\end{array}$ & $\begin{array}{l}\mathrm{R} \\
\mathrm{T} \\
\mathrm{T} \\
\mathrm{I} \\
\end{array}$ & & $\begin{array}{ll}\text { S } & \text { A } \\
\text { S } & \text { C } \\
\text { S } & \text { S } \\
\text { S } & R \\
\end{array}$ & $\begin{array}{l}\mathbf{S} \\
\mathrm{C} \\
\mathrm{D} \\
\mathrm{S} \\
\end{array}$ & $\begin{array}{l}\mathrm{T} \\
\vdots\end{array}$ & $\begin{array}{l}\dot{\mathrm{T}} \\
\dot{ }\end{array}$ & $\therefore:$ & $\therefore$ & $\begin{array}{l}\mathrm{T} \\
\mathrm{T} \\
\mathrm{S} \\
\mathrm{T} \\
\end{array}$ & & : & $:$ & $: \vdots$ & & : & $\vdots$ & $: \vdots$ & $:$ & : & $\vdots$ & $\vdots$ & & : & $\vdots$ & $\therefore$ & $\therefore:$ & : & $:$ & $:$ & $:$ & : & $:$ & $:$ & $:$ & $:$ & $::$ \\
\hline C. jubata sverdrupi & $\begin{array}{l}24 \mathrm{H}-1 \\
24 \mathrm{H}-2 \\
24 \mathrm{H}-3 \\
24 \mathrm{H}-4 \\
24 \mathrm{H}-5 \\
\end{array}$ & $\begin{array}{l}75-77 \\
75-77 \\
75-77 \\
75-77 \\
75-77 \\
\end{array}$ & $\begin{array}{l}206.4 \\
207.9 \\
209.4 \\
210.9 \\
212.4 \\
\end{array}$ & $\begin{array}{l}\text { C/G } \\
\text { C/G } \\
\text { C/G } \\
\text { F/G } \\
\text { C/G } \\
\end{array}$ & $\begin{array}{ll} & \mathrm{A} \\
\vdots & \mathrm{A} \\
\dot{\mathrm{R}} & \mathrm{A} \\
\mathbf{R} & \mathrm{A} \\
\mathrm{R} & \mathbf{A} \\
\end{array}$ & $\begin{array}{ll}\mathbf{A} & \mathbf{C} \\
\mathrm{A} & \mathbf{C} \\
\mathrm{A} & \mathbf{C} \\
\mathrm{A} & \mathbf{C} \\
\mathbf{A} & \mathbf{A} \\
\end{array}$ & $\begin{array}{l}\mathrm{R} \\
\mathrm{T} \\
\mathrm{T} \mathrm{V} \\
\mathrm{T} \mathrm{V} \\
\end{array}$ & \begin{tabular}{c|c}
$D$ & \\
$F$ & \\
VD & \\
D & \\
VD & \\
\end{tabular} & $\begin{array}{ll}\mathrm{T} & \mathrm{R} \\
\mathrm{R} & \mathrm{T} \\
\mathrm{S} & \\
\mathrm{T} & \mathrm{T} \\
\mathrm{T} & \mathrm{R} \\
\end{array}$ & $\begin{array}{l}\mathrm{R} \\
\mathrm{T} \\
\mathrm{R} \\
\mathrm{R} \\
\mathrm{S}\end{array}$ & $\vdots$ & $\dot{\mathrm{T}}$ & $\begin{array}{l}: \dot{R} \\
\vdots \\
\end{array}$ & $\begin{array}{ll} & \mathrm{C} \\
\mathrm{R} & \mathrm{C} \\
\dot{0} & \mathrm{~S} \\
\dot{2} & \mathrm{C} \\
\mathrm{R} & \mathrm{A} \\
\end{array}$ & : & & $\vdots$ & $\begin{array}{l}: \vdots \\
\vdots \\
\vdots\end{array}$ & $\vdots:$ & & $\vdots$ & $\begin{array}{l}\vdots \\
\vdots \\
\end{array}$ & 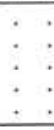 & $\vdots:$ & $\vdots$ & $\begin{array}{l}0 \\
\vdots \\
\end{array}$ & $:$ & & : & $\vdots$ & $\begin{array}{l}\vdots: \\
\vdots\end{array}$ & $\begin{array}{l}\vdots: \\
\vdots \\
\vdots\end{array}$ & : & $\begin{array}{l}: \\
\vdots \\
\end{array}$ & $\therefore:$ & $::$ & 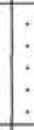 & : & $\begin{array}{l} \\
\vdots \\
\end{array}$ & $:$ & : & $\begin{array}{l}0: \\
\vdots \\
\vdots\end{array}$ \\
\hline C. sandellae & $\begin{array}{l}24 \mathrm{H}-\mathrm{CC} \\
25 \mathrm{H}-1 \\
25 \mathrm{H}-2 \\
25 \mathrm{H}-3 \\
25 \mathrm{H}-4 \\
25 \mathrm{H}-5 \\
25 \mathrm{H}-6 \\
25 \mathrm{H}-\mathrm{CC}\end{array}$ & $\begin{array}{l}75-77 \\
75-77 \\
25-27 \\
25-27 \\
25-27 \\
25-27\end{array}$ & $\begin{array}{l}213.0 \\
213.8 \\
215.3 \\
216.3 \\
217.8 \\
219.3 \\
220.8 \\
221.1\end{array}$ & $\begin{array}{l}\text { F/G } \\
\text { F/G } \\
\text { A/G } \\
\text { A/G } \\
\text { C/G } \\
\text { F/G } \\
\text { F/G } \\
\text { R/G }\end{array}$ & $\begin{array}{ll} & \text { D } \\
\text { S } & \text { D } \\
\text { S } & \text { A } \\
\text { A } & \text { A } \\
\text { A } & \text { A } \\
\text { C } & \text { A } \\
\text { C } & \text { A } \\
\text { S } & \text { A }\end{array}$ & $\begin{array}{ll}\text { D } & \text { A } \\
\text { D } & \text { S } \\
\text { A } & \text { S } \\
\text { A } & \text { A } \\
\text { A } & \text { A } \\
\text { A } & \text { R } \\
\text { A } & \text { S } \\
\text { A } & \text { S }\end{array}$ & $\begin{array}{l}\mathrm{T} \\
\mathrm{T} \\
\mathrm{T} \\
\vdots \\
\vdots \\
\mathrm{T} \\
\mathrm{T}\end{array}$ & \begin{tabular}{c|c} 
D & \\
D & \\
VD & \\
D & \\
D & \\
A & \\
vD & \\
S &
\end{tabular} & $\begin{array}{ll}\mathrm{T} & \mathrm{R} \\
\mathrm{T} & \mathrm{R} \\
\mathrm{S} & \mathrm{S} \\
\mathrm{T} & \mathrm{T} \\
\mathrm{T} & \mathrm{T} \\
\mathrm{R} & \mathrm{R} \\
\mathrm{R} & \mathrm{R} \\
\mathrm{R} & \mathrm{R}\end{array}$ & $\begin{array}{l}\mathrm{C} \\
\mathrm{T} \\
\mathrm{R} \\
\dot{\mathrm{T}} \\
\dot{\mathrm{T}} \\
\dot{ }\end{array}$ & $\begin{array}{l}\dot{\mathbf{T}} \\
\dot{\mathbf{T}} \\
\dot{ }\end{array}$ & $\begin{array}{c}\mathrm{T} \\
\mathrm{T} \\
\vdots \\
\vdots\end{array}$ & $\begin{array}{ll}\mathbf{T} & \mathbf{s} \\
\vdots & \mathbf{s} \\
\vdots & \vdots \\
\vdots & \vdots\end{array}$ & $\begin{array}{l}\text { s } \\
\text { s : } \\
\vdots \\
\vdots\end{array}$ & $\vdots$ & & : & $\begin{array}{l}: \vdots \\
\vdots \\
\vdots\end{array}$ & $\therefore:$ & & : & $\begin{array}{l}0 \\
\vdots \\
\vdots \\
\vdots\end{array}$ & $\begin{array}{l}\vdots \\
\vdots \\
\vdots\end{array}$ & $\begin{array}{l}\vdots \\
\vdots \\
\vdots\end{array}$ & : & $\begin{array}{l}0 \\
\vdots \\
\vdots\end{array}$ & $\vdots$ & & : & $\vdots$ & $\begin{array}{l}\vdots \\
\vdots \vdots \\
\vdots\end{array}$ & $\begin{array}{l}\vdots \\
\vdots \\
\vdots\end{array}$ & & $\begin{array}{l} \\
\vdots \\
\vdots \\
\vdots\end{array}$ & $\vdots$ & $:$ & 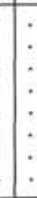 & $\vdots$ & $\begin{array}{l}: \\
\vdots \\
\vdots\end{array}$ & $\begin{array}{l}: \\
\vdots: \\
\vdots\end{array}$ & : & $\begin{array}{l} \\
\vdots \\
\vdots\end{array}$ \\
\hline
\end{tabular}


Table 3. Stratigraphic range chart for radiolaria in Hole $642 \mathrm{C}$.

\begin{tabular}{|c|c|c|c|c|c|c|c|c|c|c|c|c|c|c|c|c|c|c|c|c|c|c|c|c|c|c|c|c|c|c|c|c|c|c|c|c|c|c|}
\hline \multirow{2}{*}{$\begin{array}{l}\text { Radiolarian } \\
\text { Zonation }\end{array}$} & \multirow[b]{2}{*}{ Core-Section } & \multirow{2}{*}{$\begin{array}{c}\text { Section } \\
\text { Depth (cm) }\end{array}$} & \multirow{2}{*}{$\begin{array}{l}\text { Meters below } \\
\text { sea floor }\end{array}$} & \multirow{2}{*}{$\begin{array}{l}\text { Abundance/ } \\
\text { Preservation }\end{array}$} & \multicolumn{4}{|c|}{ 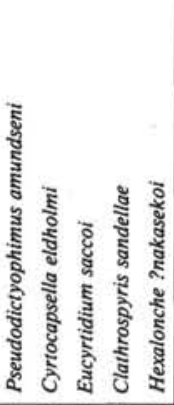 } & \multicolumn{4}{|c|}{ 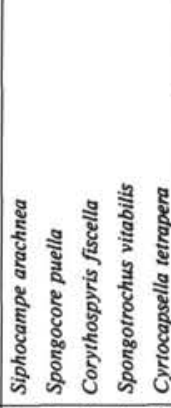 } & \multicolumn{4}{|c|}{ 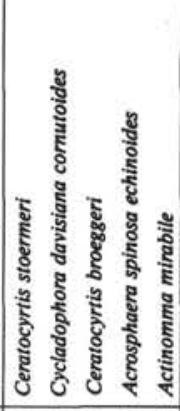 } & \multicolumn{4}{|c|}{ 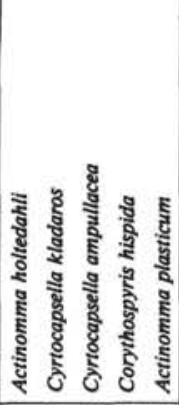 } & \multicolumn{4}{|c|}{ 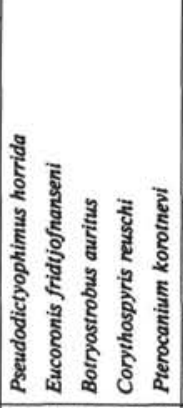 } & \multicolumn{4}{|c|}{ 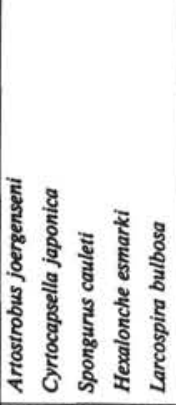 } & \multicolumn{4}{|c|}{ 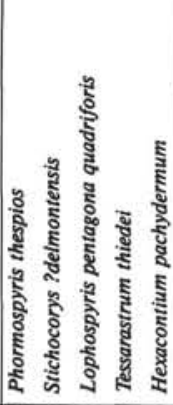 } & \multicolumn{4}{|c|}{ 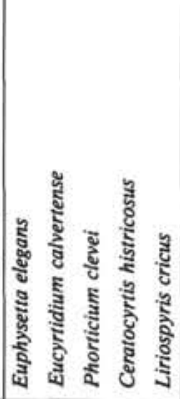 } & \multicolumn{2}{|c|}{ 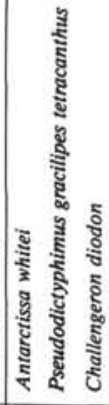 } \\
\hline & & & & & 12 & 23 & 34 & 45 & 6 & 7 & 8 & 910 & & 1112 & 131 & 141 & & 617 & 18 & 1920 & 21 & 12223 & 24 & 25 & $26 \quad 27$ & 728 & 2936 & \begin{tabular}{l|l}
303 \\
\end{tabular} & 3132 & 233 & & 35 & $36 \quad 37$ & 738 & 39 & 40 & 414 & $42 \quad 43$ \\
\hline $\begin{array}{l}\text { P. gracilipes } \\
\text { tetracanthus }\end{array}$ & $\begin{array}{l}9 \mathrm{H}-1 \\
9 \mathrm{H}-2 \\
9 \mathrm{H}-\mathrm{CC} \\
10 \mathrm{H}-3 \\
10 \mathrm{H}-4 \\
10 \mathrm{H}-5 \\
10 \mathrm{H}-6 \\
10 \mathrm{H}-\mathrm{CC} \\
11 \mathrm{H}-1 \\
11 \mathrm{H}-2 \\
11 \mathrm{H}-3 \\
\end{array}$ & $\begin{array}{l}69-71 \\
69-71 \\
63-65 \\
63-65 \\
63-65 \\
63-65 \\
70-72 \\
70-72 \\
70-72 \\
\end{array}$ & $\begin{array}{l}61.4 \\
62.9 \\
63.5 \\
67.1 \\
68.6 \\
70.1 \\
71.6 \\
73.0 \\
73.7 \\
75.2 \\
76.7 \\
\end{array}$ & $\begin{array}{l}\mathrm{F} / \mathrm{G} \\
\mathrm{A} / \mathrm{M} \\
\mathrm{A} / \mathrm{M} \\
\mathrm{C} / \mathrm{M} \\
\mathrm{C} / \mathrm{M} \\
\mathrm{A} / \mathrm{M} \\
\mathrm{A} / \mathrm{M} \\
\mathrm{A} / \mathrm{P} \\
\mathrm{F} / \mathrm{M} \\
\mathrm{F} / \mathrm{M} \\
\mathrm{F} / \mathrm{M} \\
\end{array}$ & $\begin{array}{l}: \\
\vdots \\
\vdots \\
\vdots\end{array}$ & 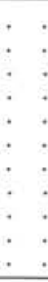 & $\begin{array}{l}: \\
\vdots \\
\vdots\end{array}$ & $\begin{array}{l}\vdots \\
\vdots \\
\vdots \\
\vdots\end{array}$ & $\begin{array}{l}: \\
\vdots \\
\text { R } \\
\dot{\mathrm{R}} \\
\mathrm{R} \\
\mathrm{R} \\
\end{array}$ & $\begin{array}{l}: \\
\vdots \\
\vdots \\
:\end{array}$ & $\begin{array}{l}\vdots \\
\vdots \\
\vdots \\
\vdots\end{array}$ & $\begin{array}{l}\vdots \\
\vdots \\
\vdots \\
\vdots\end{array}$ & & $\begin{array}{l}: \\
\vdots \\
\vdots \\
\vdots\end{array}$ & $\begin{array}{l}: \\
\vdots \\
\vdots \\
\vdots \\
\vdots \\
\vdots\end{array}$ & $\begin{array}{l}: \\
\vdots \\
\vdots \\
\vdots\end{array}:$ & & $\begin{array}{l}\vdots \\
\vdots \\
\vdots \\
\vdots\end{array}$ & $\begin{array}{l}: \\
\vdots \\
\vdots \\
\vdots \\
\vdots\end{array}$ & $\begin{array}{l}\vdots \\
\vdots \\
\vdots\end{array}$ & : & $\begin{array}{cc}: & \mathbf{R} \\
\mathbf{R} \\
\vdots \\
\mathbf{R} \\
\mathbf{S} \\
\mathbf{R} \\
\vdots \\
\vdots & \vdots \\
\vdots & \vdots \\
& : \\
\end{array}$ & $\begin{array}{l}: \\
\vdots \\
\vdots \\
:\end{array}$ & $\begin{array}{ll}\vdots \\
\vdots \\
\vdots \\
\vdots\end{array}$ & $\begin{array}{l}\mathrm{T} \\
\mathrm{T} \\
\mathrm{T}\end{array}$ & $\begin{array}{l}: \\
\vdots \\
\vdots \\
:\end{array}$ & $\begin{array}{l}\vdots \\
\vdots \\
\vdots \\
\vdots\end{array}$ & $:$ & $\begin{array}{l}\vdots \\
\vdots \\
\vdots \\
\vdots \\
\vdots \\
\vdots\end{array}$ & $\begin{array}{l}\text { S } \\
\text { S } \\
\text { S } \\
\text { S } \\
\text { S } \\
\text { S } \\
\text { S } \\
\text { C } \\
\text { S } \\
\text { C } \\
\end{array}$ & $\begin{array}{l}: \\
\vdots \\
\vdots \\
\vdots \\
:\end{array}$ & 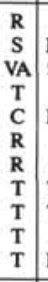 & 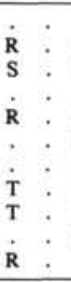 & $\begin{array}{l}\dot{T} \\
R \\
\dot{R} \\
R \\
R \\
R \\
R \\
R \\
R \\
.\end{array}$ & $\begin{array}{l}\mathbf{R} \\
\mathrm{S} \\
\mathrm{S} \\
\mathbf{R} \\
\mathrm{R} \\
\mathrm{R} \\
\mathrm{R} \\
\mathbf{R} \\
\mathrm{R} \\
\dot{\mathrm{S}} \\
\end{array}$ & $\begin{array}{l}: \\
\vdots \\
\vdots \\
: \\
:\end{array}$ & $\begin{array}{ll}\text { T } & \text { S } \\
\text { S } & \text { S } \\
\text { R } & R \\
\text { S } & R \\
\text { S } & \text { S } \\
\text { C } & T \\
\text { A } & T \\
\text { A } & \text { C } \\
\text { A } & \text { S } \\
\text { C } & \text { C } \\
\end{array}$ & $\begin{array}{l}\text { S } \\
\text { s } \\
\text { R } \\
\text { R } \\
\text { R } \\
\text { S } \\
\text { T } \\
\text { T } \\
\text { C } \\
\text { s } \\
\text { C }\end{array}$ \\
\hline A. whitei & $\begin{array}{l}11 \mathrm{H}-4 \\
11 \mathrm{H}-5 \\
11 \mathrm{H}-6 \\
11 \mathrm{H}-\mathrm{CC} \\
12 \mathrm{H}-1 \\
12 \mathrm{H}-2 \\
12 \mathrm{H}-3 \\
12 \mathrm{H}-4 \\
12 \mathrm{H}-5 \\
12 \mathrm{H}-6 \\
12 \mathrm{H}-\mathrm{CC} \\
\end{array}$ & $\begin{array}{l}70-72 \\
70-72 \\
70-72 \\
\\
68-70 \\
68-70 \\
68-70 \\
68-70 \\
68-70 \\
68-70\end{array}$ & $\begin{array}{l}78.2 \\
79.7 \\
81.2 \\
82.5 \\
83.2 \\
84.7 \\
88.2 \\
87.2 \\
89.2 \\
99.7 \\
92.0 \\
\end{array}$ & $\begin{array}{l}\mathrm{R} / \mathrm{P} \\
\mathrm{A} / \mathrm{M} \\
\mathrm{A} / \mathrm{M} \\
\mathrm{C} / \mathrm{M} \\
\mathrm{C} / \mathrm{M} \\
\mathrm{A} / \mathrm{M} \\
\mathrm{A} / \mathrm{G} \\
\mathrm{A} / \mathrm{G} \\
\mathrm{A} / \mathrm{M} \\
\mathrm{C} / \mathrm{M} \\
\mathrm{A} / \mathrm{M} \\
\end{array}$ & $\begin{array}{l}: \\
\vdots \\
\vdots \\
\vdots\end{array}$ & 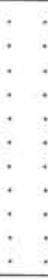 & & $\begin{array}{l}\vdots \\
\vdots \\
\vdots\end{array}$ & $\begin{array}{l}\text { VA } \\
\dot{0} \\
\dot{\mathrm{D}} \\
\dot{\mathrm{R}} \\
\dot{\mathrm{S}}\end{array}$ & $\begin{array}{l}: \\
\vdots \\
:\end{array}$ & $\begin{array}{l}\vdots \\
\vdots \\
\vdots \\
\vdots\end{array}$ & $\begin{array}{l}: \\
\vdots:\end{array}$ & & $\begin{array}{l}\vdots \\
\vdots \\
\vdots\end{array}$ & $\begin{array}{l}0 \\
\vdots \\
\vdots \\
\vdots \\
\vdots\end{array}$ & $: \vdots$ & & $\begin{array}{l}\vdots \\
\vdots \\
\vdots\end{array}$ & $\begin{array}{l}: \\
\vdots \\
\vdots \\
\vdots\end{array}$ & $\begin{array}{l}\therefore \\
\vdots \\
\vdots \\
\vdots\end{array}$ & : & $\begin{array}{cc}: & \text { D } \\
\text { D } & \text { VA } \\
: & \text { D } \\
\vdots & \vdots \\
: & \text { C } \\
: & \text { D } \\
\end{array}$ & $\begin{array}{l}: \\
\vdots \\
\vdots \\
:\end{array}$ & $\begin{array}{l}\vdots \\
\vdots \\
\vdots \\
\vdots \\
\mathrm{T} \\
\vdots\end{array}$ & $\begin{array}{l}\vdots \\
\vdots \\
\vdots \\
\vdots\end{array}$ & $\begin{array}{l}: \\
: \\
: \\
\vdots\end{array}$ & $\begin{array}{l}: \\
\vdots \\
\vdots\end{array}$ & $:$ & $\begin{array}{l}\vdots \\
\vdots \\
\vdots \\
\vdots \\
\\
\end{array}$ & $\begin{array}{l}: \\
\vdots \\
\dot{C} \\
\text { T } \\
\text { s } \\
\text { S } \\
\text { A }\end{array}$ & $\begin{array}{l}: \\
\vdots \\
\vdots \\
\vdots \\
\vdots \\
\mathrm{T}\end{array}$ & $\begin{array}{l} \\
\vdots \\
\mathrm{R} \\
\mathrm{S} \\
\vdots \\
\vdots \\
\vdots\end{array}$ & $\begin{array}{ll} & \vdots \\
\vdots & \vdots \\
\vdots & \vdots \\
\vdots & \mathrm{T} \\
\mathrm{R} & \mathrm{T} \\
\vdots & \mathrm{T}\end{array}$ & $\begin{array}{l}R \\
\text { S } \\
\text { R } \\
\text { R } \\
\text { R } \\
C \\
C \\
C \\
C \\
\text { A } \\
\text { R } \\
\text { A }\end{array}$ & $\begin{array}{l}R \\
\text { S } \\
C \\
\text { VA } \\
\text { S } \\
\text { S } \\
\dot{A} \\
\text { R } \\
\dot{R}\end{array}$ & $\begin{array}{l}: \\
\vdots \\
\vdots \\
:\end{array}$ & $\begin{array}{l}\text { S } \\
\text { VA } \\
\text { A } \\
\text { C } \\
\text { S } \\
\text { A } \\
\text { C } \\
\text { A } \\
\text { R } \\
\text { C } \\
\text { C }\end{array}$ & $:$ \\
\hline L. cricus & $\begin{array}{l}13 \mathrm{H}-1 \\
13 \mathrm{H}-2 \\
13 \mathrm{H}-\mathrm{CC} \\
14 \mathrm{H}-1 \\
14 \mathrm{H}-2\end{array}$ & $\begin{array}{l}70-72 \\
70-72 \\
\end{array}$ & $\begin{array}{l}92.7 \\
94.2 \\
95.7 \\
102.2 \\
103.7\end{array}$ & $\begin{array}{l}\mathrm{A} / \mathrm{G} \\
\mathrm{A} / \mathrm{G} \\
\mathrm{A} / \mathrm{G} \\
\mathrm{C} / \mathrm{G} \\
\mathrm{C} / \mathrm{G} \\
\end{array}$ & & $: \vdots$ & & $\therefore$ & : & : & $:$ & $\therefore:$ & & $\therefore:$ & $:$ & $: \vdots$ & & : & $:$ & & : & $\begin{array}{l}: \mathrm{S} \\
: \mathrm{S} \\
: \mathrm{C} \\
\mathrm{C} \\
\end{array}$ & : & $\begin{array}{ll} & \\
\vdots & \\
\end{array}$ & $\begin{array}{l}\mathrm{T} \\
\vdots \\
\vdots\end{array}$ & $:$ & $\therefore:$ & : & $\begin{array}{l}\mathbf{T} \\
\vdots \\
\vdots \\
\end{array}$ & s. & $\begin{array}{l}\text { A } \\
\text { A } \\
\text { VA } \\
\text { A } \\
\text { VA }\end{array}$ & \begin{tabular}{l|}
$\mathrm{T}$ \\
$\vdots$ \\
$\vdots$ \\
\end{tabular} & $\begin{array}{l}\mathrm{T} \\
\vdots \\
\vdots \\
\end{array}$ & $\begin{array}{l}S \\
C \\
\dot{C} \\
R\end{array}$ & $\begin{array}{l}\mathbf{R} \\
\mathbf{R} \\
\mathbf{R} \\
\mathbf{R} \\
\mathbf{R} \\
\end{array}$ & \begin{tabular}{l|}
$R$ \\
$S$ \\
$S$ \\
$S$ \\
$S$ \\
\end{tabular} & $\begin{array}{ll}\text { A } & : \\
\text { T } & \vdots \\
\vdots & \vdots \\
\end{array}$ & \\
\hline T. thiedei & $\begin{array}{l}14 \mathrm{H}-3 \\
14 \mathrm{H}-4 \\
14 \mathrm{H}-5 \\
14 \mathrm{H}-6 \\
14 \mathrm{H}-7 \\
14 \mathrm{H}-\mathrm{CC} \\
15 \mathrm{H}-1 \\
15 \mathrm{H}-2 \\
15 \mathrm{H}-3 \\
15 \mathrm{H}-4 \\
15 \mathrm{H}-5 \\
15 \mathrm{H}-6\end{array}$ & $\begin{array}{l}70-72 \\
70-72 \\
24-26 \\
24-26 \\
24-26 \\
25-27 \\
25-27 \\
25-27 \\
25-27 \\
25-27 \\
25-27\end{array}$ & $\begin{array}{l}105.2 \\
106.7 \\
107.7 \\
109.2 \\
110.7 \\
111.0 \\
111.3 \\
112.8 \\
114.3 \\
115.8 \\
117.3 \\
118.8\end{array}$ & $\begin{array}{l}\text { C/G } \\
\text { F/M } \\
\text { F/M } \\
\text { F/M } \\
\text { R/M } \\
\text { A/M } \\
\text { F/M } \\
\text { A/M } \\
\text { F/M } \\
\text { C/M } \\
\text { A/M } \\
\text { C/M }\end{array}$ & $\begin{array}{l}: \\
\vdots \\
\vdots \\
\vdots \\
\vdots\end{array}$ & $\begin{array}{l}: \\
\vdots \\
\vdots \\
\vdots\end{array}$ & $:$ & $\begin{array}{l}\vdots \\
\vdots \\
\vdots\end{array}$ & $\begin{array}{l} \\
: \\
: \\
\dot{R} \\
\mathbf{R} \\
\mathbf{R} \\
\mathbf{R} \\
\text { S } \\
\text { R }\end{array}$ & $\begin{array}{l}: \\
: \\
: \\
:\end{array}$ & $\begin{array}{l}: \\
\vdots \\
\vdots \\
\vdots\end{array}$ & $\begin{array}{l}\therefore \\
\vdots \\
\vdots \\
\vdots \\
\vdots \\
\vdots\end{array}$ & & $\begin{array}{l}\vdots \\
\vdots \\
\vdots\end{array} \vdots$ & $\begin{array}{l}: \\
: \\
: \\
: \\
:\end{array}$ & 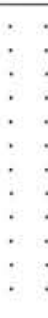 & & 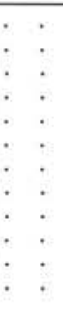 & $\begin{array}{l}: \\
: \\
\vdots \\
: \\
:\end{array}$ & $\begin{array}{l}\cdot \\
\vdots \\
\vdots\end{array}$ & : & 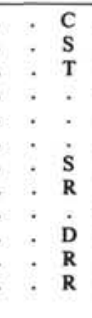 & $\begin{array}{l}: \\
\vdots \\
\vdots \\
\dot{\mathrm{R}} \\
\mathrm{T} \\
\vdots\end{array}$ & $\begin{array}{l}: \\
\vdots \\
\vdots \\
\vdots \\
\vdots \\
:\end{array}$ & $\begin{array}{l}: \\
\vdots \\
\vdots\end{array} \vdots$ & $\begin{array}{l}: \\
: \\
: \\
:\end{array}$ & $\begin{array}{l}: \\
\vdots \\
\vdots\end{array}$ & . & 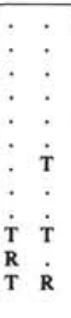 & $\begin{array}{l}\mathrm{T} \\
\vdots \\
\vdots \\
\vdots \\
\vdots\end{array}$ & $\begin{array}{c}\text { VA } \\
\text { VA } \\
\text { A } \\
\text { A } \\
\text { A } \\
\text { VA } \\
\text { S } \\
\text { D } \\
\text { T } \\
\text { VA } \\
\text { VA } \\
\text { VA }\end{array}$ & $\begin{array}{l}\mathrm{T} \\
: \\
\dot{\mathrm{T}} \\
\mathrm{A} \\
\mathrm{R} \\
\mathrm{S} \\
: \\
: \\
:\end{array}$ & $\begin{array}{ll} & \vdots \\
\vdots & \mathrm{T} \\
\vdots & \vdots \\
& \vdots \\
\vdots & \vdots \\
\vdots\end{array}$ & $\begin{array}{c}\mathbf{R} \\
\mathbf{S} \\
\vdots \\
\vdots \\
\vdots \\
\vdots\end{array}$ & $\begin{array}{l}\text { R } \\
\vdots \\
\vdots \\
\vdots \\
\vdots \\
\vdots \\
\vdots\end{array}$ & $\begin{array}{l}: \\
: \\
\vdots \\
\vdots \\
:\end{array}$ & $\begin{array}{l}: \\
: \\
:\end{array}$ & $\therefore$ \\
\hline L. bulbosa & $\begin{array}{l}15 \mathrm{H}-7 \\
15 \mathrm{H}-\mathrm{CC}\end{array}$ & $25-27$ & $\begin{array}{l}120.3 \\
120.5 \\
\end{array}$ & $\begin{array}{l}\mathrm{A} / \mathrm{M} \\
\mathrm{F} / \mathrm{M}\end{array}$ & : & : & : & $:$ & $\begin{array}{l}\text { S } \\
\text { A } \\
\end{array}$ & : & : & $\therefore:$ & & $\therefore:$ & : & & & : : & : & & & $: \mathbf{s}$ & : & $:$ & $::$ & : & & $\begin{array}{l}\mathbf{s} \\
\mathbf{c}\end{array}$ & $\begin{array}{ll}\mathrm{T} & \mathrm{R} \\
\mathrm{T} & \\
\end{array}$ & T & : & $:$ & : : & : & & : & $:$ & \\
\hline H. esmarki & $\begin{array}{l}16 \mathrm{H}-1 \\
16 \mathrm{H}-2 \\
16 \mathrm{H}-3 \\
\end{array}$ & $\begin{array}{l}25-27 \\
25-27 \\
25-27 \\
\end{array}$ & \begin{tabular}{l|}
120.8 \\
122.3 \\
123.8 \\
\end{tabular} & $\begin{array}{l}\text { C/M } \\
\text { R//P } \\
\text { /F/P } \\
\end{array}$ & : & $\vdots:$ & : & : & $\dot{\mathrm{c}}$ & : & $:$ & $\therefore:$ & & & : & : & & $\therefore:$ & $\therefore$ & $\therefore:$ & & $\dot{\mathrm{c}}$ & : & $\begin{array}{l}\mathrm{C} \\
\dot{\mathrm{T}} \\
\end{array}$ & $\therefore:$ & $\dot{\mathrm{A}}$ & $\begin{array}{l}\mathrm{C} \\
\mathrm{C} \\
\mathrm{S}\end{array}$ & $\dot{:}$ & $: \vdots$ & $\vdots$ & : & $:$ & $\therefore:$ & $:$ & $:$ & $\therefore$ & & $:$ \\
\hline S. cauleti & $\begin{array}{l}16 \mathrm{H}-4 \\
16 \mathrm{H}-5\end{array}$ & $\begin{array}{l}25-27 \\
25-27\end{array}$ & $\begin{array}{l}125.3 \\
126.8\end{array}$ & $\begin{array}{l}\mathrm{C} / \mathrm{M} \\
\mathrm{C} / \mathrm{P}\end{array}$ & & & & & \begin{tabular}{|l|}
$\mathrm{D}$ \\
$\mathrm{A}$
\end{tabular} & : & $\vdots$ & $\therefore:$ & & & $\dot{.}$ & & & & & & & $\therefore$ & & & & $\begin{array}{l}\text { VA } \\
\text { R }\end{array}$ & & & $\therefore$ & & : & $: 1$ & & : & & & & \\
\hline
\end{tabular}




\begin{tabular}{|c|c|c|c|c|c|c|c|c|c|c|c|c|c|c|c|c|c|c|c|c|c|c|c|c|c|c|c|c|c|c|c|c|c|c|c|c|c|c|c|c|c|c|c|c|}
\hline C. reuschi & $\begin{array}{l}16 \mathrm{H}-6 \\
16 \mathrm{H}-\mathrm{CC} \\
17 \mathrm{H}-1\end{array}$ & $25-27$ & $\begin{array}{l}128.3 \\
129.9 \\
130.3 \\
\end{array}$ & $\begin{array}{l}\mathrm{A} / \mathrm{M} \\
\mathrm{A} / \mathrm{M} \\
\mathrm{F} / \mathrm{M}\end{array}$ & $:$ & $\vdots:$ & $\therefore$ & $\begin{array}{l}\dot{\mathrm{C}} \\
\mathrm{T}\end{array}$ & $\begin{array}{l}\text { D } \\
\text { D } \\
\text { A } \\
\end{array}$ & $\vdots$ & : & : & $\vdots$ & $:$ & : & $:$ & : : & & $:$ & & & : : & & & $\begin{array}{l}\mathrm{S} \\
\mathrm{C} \\
\mathrm{s}\end{array}$ & $\begin{array}{l}\mathrm{R} \\
\mathrm{C} \\
\mathrm{S}\end{array}$ & : & : & $\begin{array}{l}\mathrm{S} \\
\mathrm{T} \\
\text {. }\end{array}$ & & & $\therefore$ & : & : & $:$ & : & : & : & : & : & & : & $:$ & : \\
\hline $\begin{array}{l}\text { E. fridtjofnanseni/ } \\
\text { C. reuschi }\end{array}$ & $\begin{array}{l}17 \mathrm{H}-2 \\
17 \mathrm{H}-3 \\
17 \mathrm{H}-4 \\
17 \mathrm{H}-5 \\
17 \mathrm{H}-6 \\
17 \mathrm{H}-\mathrm{CC} \\
18 \mathrm{H}-1 \\
18 \mathrm{H}-2 \\
18 \mathrm{H}-3 \\
18 \mathrm{H}-4 \\
18 \mathrm{H}-5 \\
18 \mathrm{H}-6 \\
18 \mathrm{H}-\mathrm{CC} \\
19 \mathrm{H}-1 \\
19 \mathrm{H}-2 \\
\end{array}$ & $\begin{array}{l}25-27 \\
25-27 \\
25-27 \\
25-27 \\
25-27 \\
25-27 \\
25-27 \\
25-27 \\
25-27 \\
25-27 \\
25-27 \\
25-27 \\
25-27 \\
\end{array}$ & $\begin{array}{l}131.8 \\
133.3 \\
134.8 \\
136.3 \\
137.8 \\
138.7 \\
139.8 \\
142.3 \\
142.8 \\
144.3 \\
145.8 \\
147.3 \\
148.2 \\
149.3 \\
150.8 \\
\end{array}$ & $\begin{array}{l}\text { F/M } \\
\text { F/G } \\
\text { F/G } \\
\text { C/G } \\
\text { C/G } \\
\text { F/M } \\
\text { A/G } \\
\text { C/G } \\
\text { C/G } \\
\text { F/G } \\
\text { F/G } \\
\text { F/G } \\
\text { F/G } \\
\text { R/G } \\
\text { F/G } \\
\end{array}$ & $\begin{array}{l}: \\
\vdots \\
\vdots \\
\vdots \\
\vdots \\
: \\
:\end{array}$ & 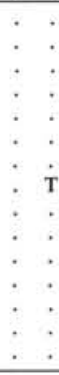 & $\begin{array}{l}\mathrm{T} \\
\mathrm{R} \\
\mathrm{S} \\
\mathrm{R} \\
\mathrm{R} \\
\mathrm{S} \\
\mathrm{C} \\
\mathrm{S} \\
\mathrm{S} \\
\mathrm{C} \\
\mathrm{A} \\
\mathrm{R} \\
\mathrm{C} \\
\mathrm{S} \\
\mathrm{C}\end{array}$ & $\begin{array}{l}\text { S } \\
\text { S } \\
\vdots \\
\vdots \\
\vdots \\
\text { R } \\
\text { R } \\
\text { T } \\
\text { T } \\
\text { A }\end{array}$ & $\begin{array}{l}\text { R } \\
\text { S } \\
\text { S } \\
\text { T } \\
\text { T } \\
\text { C } \\
\vdots \\
\vdots \\
\vdots \\
\vdots \\
\text { T }\end{array}$ & $\begin{array}{l}\vdots \\
\vdots \\
\vdots \\
\vdots \\
\vdots\end{array}$ & $\begin{array}{l}\dot{0} \\
\vdots \\
\dot{\mathrm{T}} \\
\dot{\mathrm{T}} \\
\dot{\mathrm{T}} \\
\dot{\mathrm{T}}\end{array}$ & $:$ & $\begin{array}{l} \\
\vdots \\
\vdots \\
\vdots \\
\vdots \\
\vdots\end{array}$ & $\begin{array}{l}\vdots \\
\vdots \\
\vdots \\
\vdots \\
\vdots\end{array}$ & : & $:$ & 5 & & $\begin{array}{l}\dot{C} \\
\mathrm{R} \\
\mathrm{A} \\
\mathrm{T} \\
\vdots \\
\vdots \\
\vdots \\
\vdots\end{array}$ & : & $\begin{array}{l}0 \\
\vdots \\
\vdots\end{array}$ & $\begin{array}{l}\vdots \\
\vdots \\
\vdots \\
\vdots \\
\vdots \\
\vdots \\
\end{array}$ & 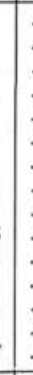 & $\begin{array}{l}\dot{S} \\
\mathrm{C} \\
\mathrm{S} \\
\mathrm{C} \\
\mathrm{A} \\
\mathrm{A}\end{array}$ & $\begin{array}{l}\text { A } \\
\text { S } \\
\text { D } \\
\text { R } \\
\text { C } \\
\text { T } \\
\text { T } \\
\text { T } \\
\text { T } \\
\text { T } \\
\text { S } \\
\text { T }\end{array}$ & $\begin{array}{l}\mathrm{D} \\
\mathrm{A} \\
\mathrm{C} \\
\mathrm{C} \\
\mathrm{S} \\
\mathrm{R} \\
\mathrm{S} \\
\mathrm{R} \\
\mathrm{R} \\
\mathrm{T} \\
\mathrm{T} \\
\mathrm{T} \\
\mathrm{T} \\
\mathrm{T} \\
\mathrm{T}\end{array}$ & $\begin{array}{l}\text { S } \\
\mathbf{R} \\
\mathbf{T} \\
\vdots \\
\vdots \\
\vdots \\
\vdots\end{array}$ & $\begin{array}{l}: \\
\text { T } \\
\vdots \\
\vdots \\
\vdots \\
\vdots\end{array}$ & $\begin{array}{l}: \\
\vdots \\
\vdots \\
\vdots\end{array}$ & $\begin{array}{l}: \\
\vdots \\
\vdots\end{array}$ & $\begin{array}{l}: \\
\vdots \\
\vdots\end{array}$ & $\begin{array}{l}: \\
: \\
: \\
\vdots \\
: \\
:\end{array}$ & $\begin{array}{l}: \\
\vdots \\
\vdots \\
\vdots \\
:\end{array}$ & $\begin{array}{l}: \\
\vdots \\
\vdots \\
\vdots\end{array}$ & $\begin{array}{l}\vdots \\
\vdots \\
\vdots \\
\vdots \\
\vdots\end{array}$ & $\begin{array}{l}\vdots \\
\vdots \\
\vdots \\
\vdots \\
\vdots \\
\vdots \\
\vdots\end{array}$ & $\begin{array}{l}\vdots \\
\vdots \\
\vdots \\
\vdots \\
\vdots\end{array}$ & $\begin{array}{l}0 \\
\vdots \\
\vdots \\
\vdots\end{array}$ & $\begin{array}{l}\text { : } \\
\vdots \\
\vdots \\
\vdots\end{array}$ & : & $\begin{array}{l}\text { : } \\
\vdots \\
\vdots \\
\vdots\end{array}$ & $\frac{}{\vdots}:$ & $\begin{array}{l} \\
\vdots \\
\vdots \\
\vdots \\
\vdots\end{array}$ & $\begin{array}{l}\text { : } \\
\vdots \\
\vdots \\
\vdots\end{array}$ \\
\hline E. fridtjofnanseni & $\begin{array}{l}19 \mathrm{H}-3 \\
19 \mathrm{H}-4 \\
19 \mathrm{H}-5 \\
19 \mathrm{H}-6 \\
19 \mathrm{H}-7 \\
19 \mathrm{H}-\mathrm{CC} \\
20 \mathrm{H}-1 \\
20 \mathrm{H}-2\end{array}$ & $\begin{array}{l}25-27 \\
25-27 \\
25-27 \\
25-27 \\
25-27\end{array}$ & $\begin{array}{l}152.3 \\
153.8 \\
155.3 \\
156.8 \\
158.3 \\
158.5 \\
158.8 \\
160.3\end{array}$ & $\begin{array}{l}\text { F/G } \\
\text { F/M } \\
\text { R/G } \\
\text { F/G } \\
\text { R/G } \\
\text { F/G } \\
\text { F/G } \\
\text { A/M }\end{array}$ & $:$ & $\begin{array}{l}\vdots \\
\vdots \\
\vdots\end{array}$ & $\begin{array}{l}\mathrm{C} \\
\mathrm{C} \\
\mathrm{C} \\
\mathrm{R} \\
\mathrm{S} \\
\mathrm{S} \\
\mathrm{S} \\
\mathrm{S}\end{array}$ & $\begin{array}{l}\mathrm{S} \\
\mathrm{S} \\
\mathrm{T} \\
\dot{\mathrm{T}} \\
\dot{\mathrm{F}}\end{array}$ & $\begin{array}{l}\dot{\mathrm{T}} \\
\dot{\mathrm{R}} \\
\dot{\mathrm{T}} \\
\dot{\mathrm{T}}\end{array}$ & : & $\begin{array}{l}\dot{\mathrm{T}} \\
\dot{\mathrm{T}} \\
\mathrm{T} \\
\end{array}$ & : & $\begin{array}{l}\vdots \\
\vdots \\
\vdots\end{array}$ & $\vdots$ & : & $:$ & 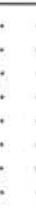 & & : & : & $\therefore$ & : & : & S & $\begin{array}{l}\text { : } \\
\vdots \\
\text { s } \\
\text { s }\end{array}$ & $\begin{array}{l}\text { : } \\
:\end{array}$ & : & $:$ & : & : & $\vdots:$ & $\begin{array}{l}: \\
: \\
\vdots \\
:\end{array}$ & : & $\begin{array}{l}: \\
\vdots\end{array}$ & $:$ & $:$ & : & : & : & : & $:$ & : & : & : \\
\hline Interzone B & $\begin{array}{l}20 \mathrm{H}-3 \\
20 \mathrm{H}-4 \\
20 \mathrm{H}-5 \\
20 \mathrm{H}-6\end{array}$ & $\begin{array}{l}25-27 \\
25-27 \\
25-27 \\
25-27 \\
\end{array}$ & $\begin{array}{l}161.8 \\
163.3 \\
164.8 \\
166.3 \\
\end{array}$ & $\begin{array}{l}\text { F/G } \\
\text { C/G } \\
\text { A/G } \\
\text { C/G } \\
\end{array}$ & $:$ & $\therefore$ & $\begin{array}{l}\mathrm{S} \\
\mathrm{S} \\
\mathrm{S} \\
\mathrm{S}\end{array}$ & $\begin{array}{l}\text { D } \\
\text { A } \\
\text { A } \\
\text { C }\end{array}$ & \begin{tabular}{|l}
$\dot{R}$ \\
T \\
T \\
\end{tabular} & : & : & : & $\dot{\mathrm{T}}$ & : & : & : & & & : & & $\begin{array}{l}\mathrm{R} \\
\mathrm{R} \\
\vdots\end{array}$ & $\begin{array}{l}\mathrm{T} \\
\mathrm{s} \\
\end{array}$ & . & : & : & : & : & : & : & : & : & $:$ & $:$ & $:$ & : & : & $:$ & : & : & : & : & : & $:$ & : \\
\hline P. horrida & $\begin{array}{l}20 \mathrm{H}-7 \\
20 \mathrm{H}-\mathrm{CC} \\
21 \mathrm{H}-1 \\
2 \mathrm{H}-2 \\
21 \mathrm{H}-3 \\
21 \mathrm{H}-4 \\
21 \mathrm{H}-5 \\
2 \mathrm{H}-6 \\
21 \mathrm{H}-7 \\
21 \mathrm{H}-\mathrm{CC} \\
22 \mathrm{H}-1 \\
22 \mathrm{H}-2 \\
22 \mathrm{H}-3 \\
\end{array}$ & $\begin{array}{l}25-27 \\
25-27 \\
25-27 \\
25-27 \\
25-27 \\
25-27 \\
25-27\end{array}$ & $\begin{array}{l}167.8 \\
168.0 \\
168.3 \\
169.8 \\
171.3 \\
172.8 \\
174.3 \\
175.8 \\
177.3 \\
177.5 \\
177.3 \\
179.3 \\
180.8 \\
\end{array}$ & $\begin{array}{l}\text { C/G } \\
\text { R/G } \\
\text { F/G } \\
\text { F/M } \\
\text { A/G } \\
\text { C/G } \\
\text { A/G } \\
\text { A/G } \\
\text { A/G } \\
\text { C/M } \\
\text { A/G } \\
\text { A/G } \\
\text { A/G } \\
\end{array}$ & $\begin{array}{l}: \\
\vdots \\
\vdots \\
\vdots \\
\vdots \\
\end{array}$ & $\begin{array}{ll}\therefore & \mathbf{S} \\
\vdots & \mathrm{R} \\
\vdots & \mathrm{C} \\
\vdots & \mathrm{C} \\
\vdots & \mathrm{S} \\
\vdots & \mathrm{S} \\
\therefore & \mathrm{S} \\
\therefore & \mathrm{C} \\
\end{array}$ & $\begin{array}{c}\mathbf{S} \\
\mathbf{R} \\
\vdots \\
\vdots \\
\vdots \\
\vdots \\
\mathrm{R}\end{array}$ & $\begin{array}{c} \\
\vdots \\
\vdots \\
\\
\text { T } \\
\text { A } \\
\text { F } \\
\text { D } \\
\text { VA } \\
\text { F } \\
\text { D } \\
\text { A } \\
\end{array}$ & $\begin{array}{l}\text { Tे } \\
\vdots \\
\vdots \\
\vdots \\
\vdots \\
\text { c } \\
\text { s }\end{array}$ & $\begin{array}{l}\vdots \\
\vdots \\
\mathrm{T} \\
\mathrm{T} \\
\mathrm{C} \\
\mathrm{T} \\
\mathrm{T} \\
\vdots \\
\vdots \\
\vdots\end{array}$ & $\vdots$ & : & $\begin{array}{l}\vdots \\
\vdots \\
\vdots \\
\vdots \\
\mathrm{T} \\
\end{array}$ & $\begin{array}{l}\vdots \\
\vdots \\
\vdots \\
\vdots\end{array}$ & $:$ & $:$ & $\begin{array}{ll} \\
\\
\\
\end{array}$ & & $\begin{array}{l} \\
\dot{S} \\
\text { R } \\
\text { S } \\
\dot{\text { T }} \\
\dot{\text { S }} \\
\text { S } \\
\text { S }\end{array}$ & , & 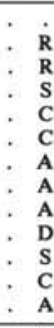 & $\begin{array}{l}\text { T } \\
\text { S } \\
\text { T } \\
\text { C } \\
\text { C } \\
\text { A } \\
\text { S } \\
\text { S } \\
\text { S } \\
\text { S } \\
\text { VA } \\
\text { A }\end{array}$ & \begin{tabular}{|l}
$\mathrm{S}$ \\
$\mathrm{T}$ \\
$\mathrm{B}$ \\
$\mathrm{S}$ \\
$\mathrm{S}$ \\
$\mathrm{R}$ \\
$\mathrm{S}$ \\
$\mathrm{S}$ \\
$\mathrm{R}$ \\
$\mathrm{S}$ \\
$\mathrm{R}$ \\
$\mathrm{R}$ \\
$\mathrm{T}$
\end{tabular} & : & $\begin{array}{l}\text { : } \\
\vdots \\
\vdots \\
:\end{array}$ & $\begin{array}{l}\text { : } \\
\vdots \\
\vdots \\
:\end{array}$ & $\begin{array}{l}: \\
\vdots \\
:\end{array}$ & $\begin{array}{l}\vdots \\
\vdots \\
\vdots\end{array}$ & $\begin{array}{l}\vdots \\
\vdots \\
\vdots\end{array}$ & $:$ & $\begin{array}{l}\vdots \\
\vdots \\
\vdots \\
\vdots\end{array}$ & $\begin{array}{l}: \\
\vdots \\
\vdots \\
\vdots \\
\vdots\end{array}$ & : & $\begin{array}{l}\vdots \\
\vdots \\
\vdots\end{array}$ & $\begin{array}{l}: \\
\vdots \\
\vdots \\
\vdots\end{array}$ & $\begin{array}{l}: \\
\vdots \\
\vdots \\
\vdots \\
\vdots \\
\end{array}$ & $\begin{array}{l}\vdots \\
\vdots \\
\vdots \\
\vdots \\
\vdots\end{array}$ & : & $\begin{array}{l}\text { : } \\
\vdots \\
:\end{array}$ & : & $\begin{array}{l}\text { : } \\
\vdots \\
:\end{array}$ & : & $\begin{array}{l}: \\
: \\
: \\
:\end{array}$ & $\begin{array}{l}\vdots \\
\vdots \\
\vdots\end{array}$ \\
\hline A. plasticum & $\begin{array}{l}22 \mathrm{H}-4 \\
22 \mathrm{H}-5 \\
22 \mathrm{H}-\mathrm{CC} \\
23 \mathrm{H}-2 \\
\end{array}$ & $\begin{array}{l}25-27 \\
25-27\end{array}$ & $\begin{array}{l}182.3 \\
183.8 \\
184.3 \\
186.0 \\
\end{array}$ & $\begin{array}{l}\mathrm{A} / \mathrm{G} \\
\mathrm{A} / \mathrm{G} \\
\mathrm{A} / \mathrm{G} \\
\mathrm{A} / \mathrm{G}\end{array}$ & $:$ & $\begin{array}{l}: \mathrm{c} \\
\vdots \\
\mathrm{T}\end{array}$ & $\begin{array}{l}\mathrm{C} \\
\dot{R} \\
\mathrm{~S}\end{array}$ & $\begin{array}{l}\vdots \\
\text { D } \\
\text { D }\end{array}$ & \begin{tabular}{|l}
$\mathrm{T}$ \\
$\dot{\mathrm{C}}$ \\
$\mathrm{D}$
\end{tabular} & $:$ & $\dot{\mathrm{T}}$ & : & $\therefore$ & : & & : & $:{ }^{5}$ & & $\begin{array}{l}\mathrm{T} \\
\mathrm{T} \\
\mathrm{S} \\
\mathrm{S}\end{array}$ & & $\begin{array}{l}\mathbf{S} \\
\mathbf{R} \\
\vdots \\
\end{array}$ & $\begin{array}{l}\mathrm{T} \\
\mathrm{T} \\
\mathrm{R} \\
\mathrm{T}\end{array}$ & : & & : & : & : & $:$ & : & : & $:$ & $:$ & : & $:$ & $:$ & $:$ & $:$ & : & : & : & $:$ & : & $:$ & $:$ \\
\hline C. kladaros - & $\begin{array}{l}23 \mathrm{H}-3 \\
23 \mathrm{H}-4 \\
23 \mathrm{H}-5 \\
23 \mathrm{H}-\mathrm{CC} \\
24 \mathrm{H}-1 \\
24 \mathrm{H}-2\end{array}$ & $\begin{array}{l}24-26 \\
24-26 \\
24-26\end{array}$ & $\begin{array}{l}187.5 \\
189.0 \\
190.5 \\
192.4 \\
192.7 \\
194.2\end{array}$ & $\begin{array}{l}\text { A/G } \\
\text { A/G } \\
\text { A/G } \\
\text { F/G } \\
\text { C/G } \\
\text { C/G }\end{array}$ & $\vdots:$ & $\begin{array}{ll}\cdot & \mathrm{R} \\
\vdots & \mathrm{T} \\
\vdots & \mathrm{S} \\
\mathrm{T} & \mathrm{T} \\
\mathrm{T} & \mathrm{T}\end{array}$ & $\begin{array}{l}\mathrm{S} \\
\mathrm{C} \\
\mathrm{R} \\
\mathrm{S} \\
\mathrm{S} \\
\mathrm{S}\end{array}$ & $\begin{array}{l}\mathrm{A} \\
\text { VD } \\
\text { D } \\
\text { A } \\
\text { D } \\
\text { VA }\end{array}$ & $\begin{array}{l}\text { S } \\
\text { D } \\
\text { A } \\
\text { C } \\
\text { S } \\
\text { C }\end{array}$ & $\begin{array}{l}\text { Tे } \\
\vdots\end{array}$ & : & : & $:$ & $\vdots$ & : & : & : & & $\begin{array}{l}\mathrm{S} \\
\mathrm{R} \\
\mathrm{C} \\
\vdots \\
\mathrm{A}\end{array}$ & & $\begin{array}{ll}\mathrm{R} & \mathrm{C} \\
\mathrm{R} & \mathrm{R} \\
\mathrm{T} & \mathrm{R} \\
\mathrm{R} & \mathrm{S} \\
\mathrm{R} & \mathbf{Z} \\
\vdots & \vdots\end{array}$ & i & & & : & : & $\vdots$ & $\vdots:$ & $\vdots$ & : & $\vdots$ & $\therefore:$ & : & $\vdots$ & $\vdots:$ & $\begin{array}{l}: \\
\vdots \\
\vdots\end{array}$ & $\vdots$ & : & : & : & $:$ & : & $:$ & $\vdots:$ \\
\hline C. broeggeri & $\begin{array}{l}24 \mathrm{H}-3 \\
24 \mathrm{H}-4\end{array}$ & $\begin{array}{l}25-27 \\
25-27\end{array}$ & $\begin{array}{l}195.7 \\
197.2\end{array}$ & $\begin{array}{l}\text { C/G } \\
\text { F/G }\end{array}$ & $\begin{array}{l}\mathrm{T} \\
\mathrm{T} \\
\end{array}$ & $\therefore \quad \mathrm{VA}^{\mathrm{T}}$ & $\begin{array}{l}R \\
\text { A }\end{array}$ & $\begin{array}{l}\text { C } \\
\text { D }\end{array}$ & $\begin{array}{l}\mathrm{C} \\
\mathrm{S}\end{array}$ & : & : & $\mathbf{R}$ & $\dot{\mathrm{T}}$ & $\dot{R}$ & $\begin{array}{l}\mathrm{T} \\
\mathrm{T} \\
\end{array}$ & R & T & & A & & $\therefore:$ & : & & & : & : & : & : & : & : & : & : & : & : & : & : & : & : & : & : & : & & : & : \\
\hline C. eldholmi & $\begin{array}{l}24 \mathrm{H}-5 \\
24 \mathrm{H}-\mathrm{CC}\end{array}$ & $25-27$ & $\begin{array}{l}198.7 \\
199.6\end{array}$ & $\begin{array}{l}\text { C/G } \\
\text { A/G }\end{array}$ & $\begin{array}{l}\mathrm{T} \\
\mathrm{S}\end{array}$ & $\begin{array}{ll}R & S \\
T & A\end{array}$ & $\begin{array}{l}\mathrm{S} \\
\mathrm{C}\end{array}$ & $\begin{array}{l}\text { D } \\
\text { C }\end{array}$ & $\begin{array}{l}\mathrm{S} \\
\mathrm{s}\end{array}$ & T & s & : & $\vdots$ & : & : & . & 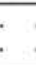 & & : & & $\therefore$ & : & & & & . & : & : & : & : & : & : & : & $\therefore$ & : & . & . & & : & . & : & & : & : \\
\hline
\end{tabular}


Dzinoridze et al. (1978), no equivalent

Sakai (1984), upper Diartus pettersoni Zone to lower Didymocyrtis antepenultimus Zone

Martini (1971), Zones NN9-NN10

Assemblage: Siphocampe arachnea, Eucyrtidium saccoi, Actinomma holtedahli (sporadic), Botryostrobus auritus (sporadic). Last occurrences of Clathrospyris sandellae and Hexalonche ?nakasekoi (consistent). First occurrence of Pterocanium korotnevi.

Occurrence:

Section 104-642B-16H-CC to Sample 104-642B-18H-4, 24-26 cm; $52.5-138.0 \mathrm{mbsf}$

Samples $104-642 \mathrm{C}-17 \mathrm{H}-3,25-27 \mathrm{~cm}$ to $-642 \mathrm{C}-19 \mathrm{H}-2,25-27 \mathrm{~cm}$; $133.3-150.8 \mathrm{mbsf}$

Samples 104-643A-11X-2, 106-108 cm to Section 104-643A-11H-CC; 93.4-100.3 mbsf

Hole 341 , Core 26 , Sect. $1,65-67 \mathrm{~cm}$ to Core 26 , CC; $352.2-361.0$ mbsf

\section{CORYTHOSPYRIS REUSCHI ZONE}

Category: Interval Zone

Definition: Interval from the last occurrence of Eucoronus fridtjofnanseni to the first occurrence of Spongurus cauleti n.sp.

Status: New zone

Age: Late Miocene; 8.1-8.3/8.6 Ma

Paleomagnetic chron: $\mathrm{C} 4 \mathrm{~A}$

Equivalent Zones:

ODP Leg 104, Initial Reports, lower Zone NSR9

DSDP Leg 38, no equivalent

Dzinoridze et al. (1978), no equivalent

Sakai (1984), mid-Didymocyrtis antepenultimus Zone

Martini (1971), upper Zone NN10 to lower Zone NN11

Assemblage: Siphocampe arachnae, Botryostrobus auritus, Hexalonche ?nakasekoi (sporadic), Pterocanium korotnevi (sporadic), Eucyrtidium saccoi (sporadic), Artostrobus joergenseni (sporadic), Cyrtocapsella japonica (only in Hole $642 \mathrm{C}$ )

Occurrence:

Samples $104-642 \mathrm{~B}-16 \mathrm{H}-3,31-33 \mathrm{~cm}$ to $-642 \mathrm{~B}-16 \mathrm{H}-7,31-33 \mathrm{~cm}$; 131.4-137.4 mbsf

Samples $104-642 \mathrm{C}-16 \mathrm{H}-6,25-27 \mathrm{~cm}$ to $-642 \mathrm{C}-17 \mathrm{H}-2,25-27 \mathrm{~cm}$; 128.3-131.8 mbsf

Samples 104-643A-10X-6, 105-107 cm to -643A-11H-1, 106-108 cm; $89.9-91.9 \mathrm{mbsf}$

Hole 341 , Core 25 , Sect. 5, 2-4 cm to CC; $338.5-342.0 \mathrm{mbsf}$

\section{SPONGURUS CAULETI ZONE}

Category: Partial Range Zone

Definition: Interval from the first occurrrence of Spongurus cauleti n. sp. to the first occurrence of Hexalonche esmarki n. sp.

Status: New zone

Age: Late Miocene; 7.7/7.9-8.1 Ma

Paleomagnetic chron: C4-C4A

Equivalent zones:

ODP Leg 104 Initial Reports, lower Zone NSR9

DSDP Vol. 38 , no equivalent

Dzinoridze et al. (1978), no equivalent

Sakai (1984), upper Didymocyrtis antepenultimus Zone

Martini (1971), Zone NN11

Assemblage: Hexalonche ?nakasekoi (sporadic), Botryostrobus auritus, Siphocampe arachnea, Pterocanium korotnevi (sporadic), Actinomma holtedahli (sporadic), Cyrtocapsella japonica (in Hole 642B only). Last occurrence of Corythospyris reuschi.

Occurrence:

Samples $104-642 \mathrm{~B}, 15 \mathrm{H}-3,27-29 \mathrm{~cm}$ to $-642 \mathrm{~B}-16 \mathrm{H}-2,31-33 \mathrm{~cm}$; $126.8-129.9 \mathrm{mbsf}$

Samples $104-642 \mathrm{C}-16 \mathrm{H}-4,25-27 \mathrm{~cm}$ to $-642 \mathrm{C}-16 \mathrm{H}-5,25-27 \mathrm{~cm}$; $125.3-126.8 \mathrm{mbsf}$

Samples 104-643A-10H-1, $105-107 \mathrm{~cm}$ to $-643 \mathrm{~A}-10 \mathrm{H}-5,105-107 \mathrm{~cm}$; $82.4-88.4 \mathrm{mbsf}$

\section{HEXALONCHE ESMARKI ZONE}

Category: Partial Range Zone

Definition: Interval from the first occurrence of Hexalonche esmarki $\mathrm{n}$. sp. to the first occurrence of Larcospira bulbosa $\mathrm{n}$. sp.

Status: New zone
Age: Late Miocene; 6.3/6.9-7.7/7.9 Ma

Paleomagnetic chron: $\mathrm{C} 4$

Equivalent zones:

ODP Leg 104 Initial Reports, upper Zone NSR9

DSDP Vol. 38 , no equivalent

Dzinoridze et al. (1978), no equivalent

Sakai (1984), upper Didymocyrtis antepenultimus to Didymocyrtis penultimus Zones

Martini (1971), Zone NN11

Assemblage: Botryostrobus auritus (sporadic) and Pterocanium korotnevi (sporadic). Last consistent occurrence of Siphocampe arachnea and last occurrence of Spongurus cauleti. First occurrences of Lophospyris pentagona quadriforis and Phormospyris thespios.

Occurrence:

Sample 104-642B-15H-2, 27-29 cm; $125.3 \mathrm{mbsf}$

Samples $104-642 \mathrm{C}-16 \mathrm{H}-1,25-27 \mathrm{~cm}$ to $-642 \mathrm{C}-16 \mathrm{H}-3,25-27 \mathrm{~cm}$; $120.8-123.8 \mathrm{mbsf}$

Samples $104-643 \mathrm{~A}-8 \mathrm{H}-1,21-23 \mathrm{~cm}$ to $-643 \mathrm{~A}-9 \mathrm{H}-\mathrm{CC}$; $70.8-81.3 \mathrm{mbsf}$

\section{LARCOSPIRA BULBOSA ZONE}

Category: Partial Range Zone

Definition: Interval from the first occurrence of Larcospira bulbosa

n. sp. to the first occurrence of Tessarastrum thiedei $\mathrm{n}$. sp.

Status: New zone

Age: Late Miocene; 6.2-6.3/6.9 Ma (interpreted)

Paleomagnetic chron: C3A (interpreted)

Remarks: The true base of this zone is not recorded in Leg 104 recovery. $L$. bulbosa must have its first occurrence in the interval between the upper part of Core 104-643A-8H, and the lowermost portion of Core $104-642 \mathrm{C}-15 \mathrm{H}$.

Equivalent zones:

ODP Leg 104 Initial Reports, lowermost Zone NSR9

DSDP Vol. 38, no equivalent

Dzinoridze et al. (1978), no equivalent

Sakai (1984), upper Didymocyrtis penultimus Zone

Martini (1971), Zone NN11

Assemblage: Siphocampe arachnea, Botryostrobus auritus, Stichocorys delmontensis. First occurrence of Phormospyris thespios.

Occurrence:

Sample $104-642 \mathrm{C}-15 \mathrm{H}-7,25-27 \mathrm{~cm}$ to $-642 \mathrm{C}-15 \mathrm{H}-\mathrm{CC} ; 120.3-120.5$ mbsf

\section{TESSARASTRUM THIEDEI ZONE}

Category: Partial Range Zone

Definition: Interval from the first occurrence of Tessarastrum thiedei n. sp. to the first occurrence of Liriospyris cricus

Status: New zone

Age: Late Miocene; 5.5-6.2 Ma

Paleomagnetic chron: $\mathrm{C} 3 \mathrm{~A}$

Equivalent Zones:

ODP Leg 104 Initial Reports, Zone NSR10

DSDP Vol. 38 , no equivalent

Dzinoridze et al. (1978), no equivalent

Sakai (1984), lower Stichocorys peregrina Zone

Martini (1971), upper Zone NN11 to lower Zone NN12

Assemblage: Stichocampe arachnea (sporadic, rare), Botryostrobus auritus (one abundance peak). Sporadic occurrences of Artostrobus joergenseni, Stichocorys delmontensis, Lophospyris pentagona quadriforis, Phorticium clevei, Ceratocyrtis histricosus, Eucyrtidium calvertense.

Occurrence:

Samples 104-642B-13H-1, 24-26 cm to $-642 \mathrm{~B}-15 \mathrm{H}-1,27-29 \mathrm{~cm}$; 104.4-123.8 mbsf

Samples $104-642 \mathrm{C}-14 \mathrm{H}-3,70-72 \mathrm{~cm}$ to $-642 \mathrm{C}-15 \mathrm{H}-6,25-27 \mathrm{~cm}$; $105.2-118.8 \mathrm{mbsf}$

Samples $104-643 \mathrm{~A}-8 \mathrm{H}-1,103-105 \mathrm{~cm}$ to $-643 \mathrm{~A}-8 \mathrm{H}-5,103-105 \mathrm{~cm}$; 63.3-69.3 mbsf

Hole 342, Core 3, Sect. 1, 100-102 cm to Sect. 4, 60-62 cm; 86.0$90.1 \mathrm{mbsf}$

\section{LIRIOSPYRIS CRICUS ZONE}

Category: Taxon Range Zone

Definition: Interval including the total statigraphic range of Liriospyris cricus. 
Status: New zone

Age: Late Miocene; 5.2-5.5 Ma

Paleomagnetic chron: $\mathrm{C} 3 / \mathrm{C} 3 \mathrm{~A}$

Equivalent Zones:

ODP Leg 104 Initial Reports, Zone NSR11

DSDP Vol. 38 , no equivalent

Dzinoridze et al. (1978), no equivalent

Sakai (1984), lower Stichocorys peregina Zone

Martini (1971), lower Zone NN12

Assemblage: Botryostrobus auritus, Hexacontium pachydermum, Phorticium clevei, and Ceratocyrtis histricosus. Sporadic occurrences of Siphocampe arachnea, Stichocorys delmontensis, Artostrobus joergenseni, and Lophospyris pentagona quadriforis. Last occurrence of Tessarastrum thiedei. First occurrence of Antarctissa whitei.

Occurrence:

Sample 104-642B-11H-7, 25-27 cm to -642B-12H-CC; $94.7-104.2$ mbsf

Sample 104-642C-13H-1, 70-72 cm to -642C-14H-2, 70-72 cm; 92.7$103.7 \mathrm{mbsf}$

\section{ANTARCTISSA WHITEI ZONE}

Category: Interval Zone

Definition: Interval from the last occurrence of Liriospyris cricus to the first occurrence of Pseudodictyophimus gracilipes tetracanthus.

Status: Originally proposed by Bjørklund (1976), emended herein.

Remarks: As originally defined, the $A$. whitei Zone was a taxon range zone, based on $A$. white $i$ and is equivalent to the interval from the upper Liriospyris cricus Zone to the top of the Pseudodictyophimus gracilipes tetracanithus Zone of the present scheme.

Age: Pliocene, 4.0-5.2 Ma

Paleomagnetic chron: $\mathrm{C} 3$

Equivalent Zones:

ODP Leg 104 Initial Reports, upper Zone NSR12 to Zone NN13

DSDP Vol. 38, lower Antarctissa whitei Zone

Dzinoridze et al. (1978), Zone XV

Sakai (1984), upper Stichocorys peregrina Zone to lower Sphaeropyle langii Zone

Martini (1971), Zones NN12-NN13

Assemblage: Siphocampe arachnae (sporadic, two abundance peaks), Botryostrobus auritus (sporadic, two abundance peaks), Phorticium clevei, Ceratocyrtis histricosus. Sporadic occurrences of Artostrobus joergenseni, Pterocanium korotnevi, Lophospyris pentagona quadriforis, Eucyrtidium calvertense, Stichocorys delmontensis, Eucyrtidium saccoi, Euphysetta elegans, Challengeron diodon.

Occurrence:

Sample 104-642B-10H-2, 25-27 cm to -642B-11H-6, 25-27 cm; 77.7$93.2 \mathrm{mbsf}$

Sample $104-642 \mathrm{C}-11 \mathrm{H}-4,70-72 \mathrm{~cm}$ to $-642 \mathrm{C}-12 \mathrm{H}-\mathrm{CC} ; 78.2-92.0$ mbsf

\section{PSEUDODICTYOPHIMUS GRACILIPES TETRACANTHUS} ZONE

Category: Partial Range Zone

Definition: Interval from the first occurrence of Pseudodicytophimus gracilipes tetracanthus to the first occurrence of Spongaster ?tetras.

Status: New zone

Remarks: The true top of the zone is not recorded in Leg 104 recovery.

Age: Pliocene; $2.9 / 3.1-4.0 \mathrm{Ma}$

Paleomagnetic chron: $\mathrm{C} 2 \mathrm{~A} / \mathrm{C} 3$

Equivalent zones:

ODP Leg 104 Initial Reports, upper Zone NSR12

DSDP Vol. 38, upper Antarctissa whitei Zone

Dzinoridze et al. (1978), Zone XV

Sakai (1984), upper Sphaeropyle langii Zone

Martini (1971), Zones NN14-NN16

Assemblage: Hexacontum pachydermum, Phorticium clevei, Eucyrtidium calvertense, Challengeron diodon, Euphysetta elegans. Sporadic occurrences of Siphocampe arachnea, Botryostrobus auritus, Artostrobus joergenseni, Stichocorys delmontensis, Lophospyris pentagona quadriforis.

Occurrence:

Samples 104-642B-9H-1, 75-77 cm to -642B-10H-1, 75-77 cm; 67.2$76.7 \mathrm{mbsf}$
Section $104-642 \mathrm{C}-9 \mathrm{H}-\mathrm{CC}$ to Sample $104-642 \mathrm{C}-11 \mathrm{H}-3,70-72 \mathrm{~cm}$; 63.5-76.7 mbsf

\section{SPONGASTER ?TETRAS ZONE}

Category: Partial Range Zone

Definition: Interval from the first occurrence of Spongaster ?tetras to the first occurrence of Cycladophora davisiana davisiana.

Status: New zone

Remarks: The true base of this zone is not recorded in Leg 104 recovery. S. ?tetras must have made its first occurrence on the Vøring Plateau after deposition of Core 104-642B-9H and before deposition of Core 104-644A-34H.

Age: Pliocene; $2.6-2.9 / 3.1 \mathrm{Ma}$

Paleomagnetic chron: $\mathrm{C} 2 \mathrm{~A}$

Equivalent zones:

ODP Leg 104 Initial Reports, upper Zone NSR12

DSDP Vol. 38, no equivalent

Dzinoridze et al. (1978), no equivalent

Sakai (1984), lower Lamprocyrtis heteroporus Zone

Martini (1971), NN16

Assemblage: Lophospyris pentagona quadriforis, Pseudodictyophimus gracilipes tetracanthus, Siphocampe arachnea, Botryostrobus auritus, Antarctissa whitei, Ceratocyrtis histricosus. Spongaster ?tetras, Hexacontium pachydermum, and Artostrobus joergenseni.

Occurrence:

Samples 104-644A-31H-3, 25-27 cm to -644A-34H-CC; $236.9-252.8$ mbsf.

\section{CYCLADOPHORA DAVISIANA DAVISIANA ZONE}

Category: Taxon Range Zone

Definition: Interval including the total stratigraphic range of Cycladophora davisiana davisiana.

Status: Originally proposed by Bjørklund (1976), emended herein

Remarks: The last occurrence of Antarctissa whitei was the boundary criterion for the base of this zone as originally defined. In its present emended sense, the zone is believed to extend up to the Holocene, but it has not been observed in Cores 104-644A-1H to $-644 \mathrm{~A}-10 \mathrm{H}$

Age: Late Pliocene-Pleistocene; 0-2.6 Ma.

Paleomagnetic chron: $\mathrm{Cl}-\mathrm{C} 2 \mathrm{~A}$

Equivalent zones:

ODP Leg 104 Initial Reports, Zone NSR13

DSDP Vol. 38, Cycladophora davisiana Zone

Dzinoridze et al. (1978), no equivalent

Sakai (1984), Lamprocyrtis heteroporus Zone to Botryostrobus aquilonaris Zone

Martini (1971), NN16-NN21

Assemblage: Pseudodictyophimus gracilipes tetracantha, Siphocampe arachnea, Botryostrobus auritus, Ceratocyrtis histricosus, and Cycladophora davisiana davisiana.

Occurrence:

Section 104-644A-11H-CC to Sample 104-644A-30H-3, 25-27 cm; 101.7-229.4 mbsf.

\section{CHRONOSTRATIGRAPHIC BOUNDARY CRITERIA}

With a few important exceptions, many of the Neogene radiolaria encountered in Leg 104 recovery are clearly distinguished taxonomically from contemporaneous temperate and tropical assemblages or have no obvious warm-water counterparts. Cosmopolitan species observed in our samples are usually rare, long-ranging, and have sporadic distributions. An example of such an occurrence is Cyrtocapsella tetrapera, which ranges discontinuously in very low frequencies down to 104-642D-10X-4, (Tables 2-5). Despite this poor record, the presence of C. tetrapera indicates that strata above its oldest occurrence must be younger than basal Miocene according to Sakai (1984), but otherwise $C$. tetrapera has little biostratigraphic potential in Leg 104 recovery. In this respect, Neogene radiolaria differ from some other fossil groups preserved in Vøring Plateau sediments (i.e., silicoflagellates), which include numerous cosmopolitan species correlatable to geographically distant localities (Ciesiel- 
ski et al., this volume; Locker and Martini, this volume). On the other hand, radiolarians are the only group of siliceous microfossils that have been examined for this volume in all of the Leg 104 holes (with the exceptions of Holes 642A and 644B) and radiolarians have been very useful for local correlation. Two radiolarians have been selected as local and tentative series/subseries boundary criteria for Leg 104 recovery in the absence of other well-established marker events.

In Eldholm, Thiede, Taylor, et al. (1987) the first occurrence of Thalassiosira oestrupii was designated as the marker event for the base of the Pliocene series. Ciesielski and Case (this volume note that this event occurs in Sections $104-642 \mathrm{C}-11 \mathrm{H}-5$ to $642 \mathrm{C}-12 \mathrm{H}-2$ ( $82.7 \mathrm{mbsf}$ ), but no detailed documentation of diatom biostratigraphy is available for this volume. Liriospyris cricus was described by Westberg-Smith and Riedel (1984) in sediments from the Rockall Plateau (Holes 81-552, 81-554, and 81$555)$, where its range is restricted to uppermost zone NN11. The last occurrence of this easily-recognized species has been selected as the criterion for recognition of the Miocene/Pliocene boundary. This event occurs in Section 104-642C-13H-1 (Table 3 , col. 40) at a depth of 92.7 mbsf, which is $10 \mathrm{~m}$ below the $T$. oestrupii datum. Although this choice is unique to this volume and must be regarded as provisional, it is consistent with the paleomagnetic stratigraphy and other biostratigraphic evidence, and we are confident that his datum conforms closely to the convention of Berggren et al. (1985).

As it has been interpreted in the biostratigraphic synthesis (Goll, this volume), the recovery in Cores 104-643A-15H to $-643 \mathrm{~A}-18 \mathrm{X}$ includes a continuous sedimentary record across the lower/middle Miocene boundary (16.3 Ma, according to Berggren et al., 1985). The first occurrence of Denticulopsis lauta (diatom) was chosen as the marker event for this boundary in Eldholm, Thiede, Taylor, et al. (1987) but we have been forced to find another criterion for reasons mentioned above. The first occurrence of Ceratocyrtis manumi (interpreted age, 16.0-16.2 $\mathrm{Ma}$ ) is arbitrarily selected as the local marker for the base of the middle Miocene. This event occurs in 643A-17X-CC (148.5 mbsf), which is $13.5 \mathrm{~m}$ below the first occurrence of $D$. lauta (Sections 104-643A-15X-4 to $-643 \mathrm{~A}-15 \mathrm{X}-5$ ) as noted by Ciesielski and Case (this volume). Because of the lack of confirming evidence, the $C$. manumi datum must be regarded as having low reliability, and subseries nomenclature for this portion of Hole 643A recovery should be used only in the informal sense (lower Miocene, middle Miocene).

\section{SITE SUMMARIES}

Site 642

Lat. $67^{\circ} 13.5^{\prime} \mathrm{N}$

Long. $2^{\circ} 55.7^{\prime} \mathrm{E}$

Water depth: $1286 \mathrm{~m}$

Five holes were drilled at this site (Holes 642A-642E), and the geologic column was continuously cored to a total depth of 1229.4 mbsf. The lower $951.9 \mathrm{~m}$ of the recovery, Section 642D$11 \mathrm{X}-2$ to Core $642 \mathrm{E}-107 \mathrm{R}$, penetrated Eocene pyroclastic muds and conglomerate overlying Eocene basaltic flows and dykes with minor tuff and shale intercalations. These sequences have not been thoroughly searched for radiolarians under the assumption that the probable shallow water facies, thermal history, and deep burial preclude silica preservation in the opal-A phase. Samples 104-642E-107R-4, 35-37 cm and, 140-142 cm were examined but proved to be barren of all siliceous microfossils. Specimens from the interval in the upper basalt series referred to as bearing "Larcoida" (Eldholm, Thiede, Taylor, et al., 1987, p. 147) have not been observed by the authors. Future investigations should include an analysis of samples from Cores 104-642E-35R, -42R, and -55R, where Eocene diatoms and sili- coflagellates have been reported (Eldholm, Thiede, Taylor, et al., 1987). Glauconite-replaced casts of radiolarians are sparse occurrences in Core 104-642D-12X, but their age is indeterminant.

The base of the biosiliceous interval at Site 642 is very sharp in Section 104-642D-11X-1, and preservation of radiolarians above this horizon is generally characterized as good, with a few exceptions in Cores 104-642D-9X and -10X. Many samples in Hole $642 \mathrm{D}$ contain abundant radiolarians, although the masking effect of the more numerous diatoms and volcanic glasses is variable. The stratigraphic ranges of 23 taxa are documented in 62 samples from Hole 642D (Table 4). We regard the interval 104-642D-11X-1 to -8 X-4 as having an older Miocene age than other Leg 104 biosiliceous recovery, although this interpretation is disputed by Manum et al. (this volume). The Actinomma henningsmoeni Zone and Eucyrtidium saccoi Zone of Hole 642D are not present in Hole 643A. At least three hiatuses in Hole $642 \mathrm{D}$ are indicated by radiolarian biostratigraphy. The abrupt first occurrence of Gondwanaria japonica kiaeri in Section 104642 D- $8 X-3$ suggests that sediments containing evolutionary intermediate morphs are missing. The G. japonica kiaeri Zone ranges from Sections 104-642D-8X-3 to $-6 \mathrm{X}-1$ and is $22.7 \mathrm{~m}$ thick. In terms of evolution the oldest members of the Clathrospyris sandellae lineage are not recorded in Hole 642D, and a hiatus is presumed to occur at the top of the $G$. japonica kiaeri Zone. The interval 104-642D-5X-CC to -3X-3 (25.6 m thick) is assigned to the Clathrospyris sandellae Zone, Corythospyris jubata sverdrupi Zone and Pseudodictyophimus amundseni Zone. The thickness of this sequence is only $38 \%$ of the coeval sequence in Hole $643 \mathrm{~A}$, and Hole $642 \mathrm{D}$ recovery may have been condensed by additional small hiatuses that we do not attempt to resolve in this paper. Three zones (Spongotrachus vitabilis, Cycladophora davisiana cornutoides, and Ceratocyrtis manumi are missing above Section 104-642D-3X-4, and a major hiatus must exist between our samples from Sections 104- 642D-3X, -2 and -3. Interestingly, Pseudodictyophimus amundseni ranges up into the base of the Cyrtocapsella eldholmi Zone. The Ceratocyrtis broeggeri Zone is represented only in Section 104-642D$2 \mathrm{X}-6$, and Ceratocyrtis stoermeri is not observed. We infer an unconformity to explain the thin C. broeggeri Zone at Site 642, which is $19.1 \mathrm{~m}$ thick in Hole 643A. The youngest recovery of Core 104-642D-2X occupies the basal Ceratocyrtis ampullacea Subzone.

The preservation and abundance of radiolarians varies considerably in Holes 642B and 642C. Preservation is generally good below Sections 104-642B-16H-CC and 104-642C-17H-2. Abundances are classified as rare to common throughout much of these intervals, although radiolarians are recorded as abundant in many samples from Cores $104-642 \mathrm{~B}-20 \mathrm{H}$ to $-22 \mathrm{H}$ and $104-642 \mathrm{C}-21 \mathrm{H}$ to $-23 \mathrm{H}$. Above Cores $104-642 \mathrm{~B}-17 \mathrm{H}$ and $104-$ $642 \mathrm{C}-19 \mathrm{H}$ the quality of preservation degrades to only moderate and occasionally poor in Cores 104-642B-13H and 104$642 \mathrm{C}-16 \mathrm{H}$. Other siliceous microfossils become progressively less abundant in younger sediments, and conversely, radiolarians have higher frequencies of occurrence in our processed residues. Radiolarians are classified as abundant in Cores 104$642 \mathrm{~B}-11 \mathrm{H}$ to $-12 \mathrm{H}$ and $104-642 \mathrm{C}-12 \mathrm{H}$ to $-13 \mathrm{H}$. Above Sections 104-642B-9H-1 and 104-642C-9H-CC, radiolarians are very rare or absent, and it has not been possible to analyze these younger intervals biostratigraphically. Abundant well-preserved radiolarians reappear only in the top of Section 104-642A-1H-1.

The stratigraphic ranges of 48 taxa have been documented in 117 samples from Hole 642B (Table 2), and 43 taxa have been documented in 103 samples from Hole $642 \mathrm{C}$ (Table 3 ). The interval below Section 104-642B-23H-3 penetrates a similar $C$. sandellae Zone to $P$. amundseni Zone sequence, as was recovered in Sections 104-642D-3X-3 to -5-CC, although the interval in Hole 642B appears to be slightly less complete (19.8 m thick). 
Table 4. Stratigraphic range chart for radiolaria in Hole 642D.

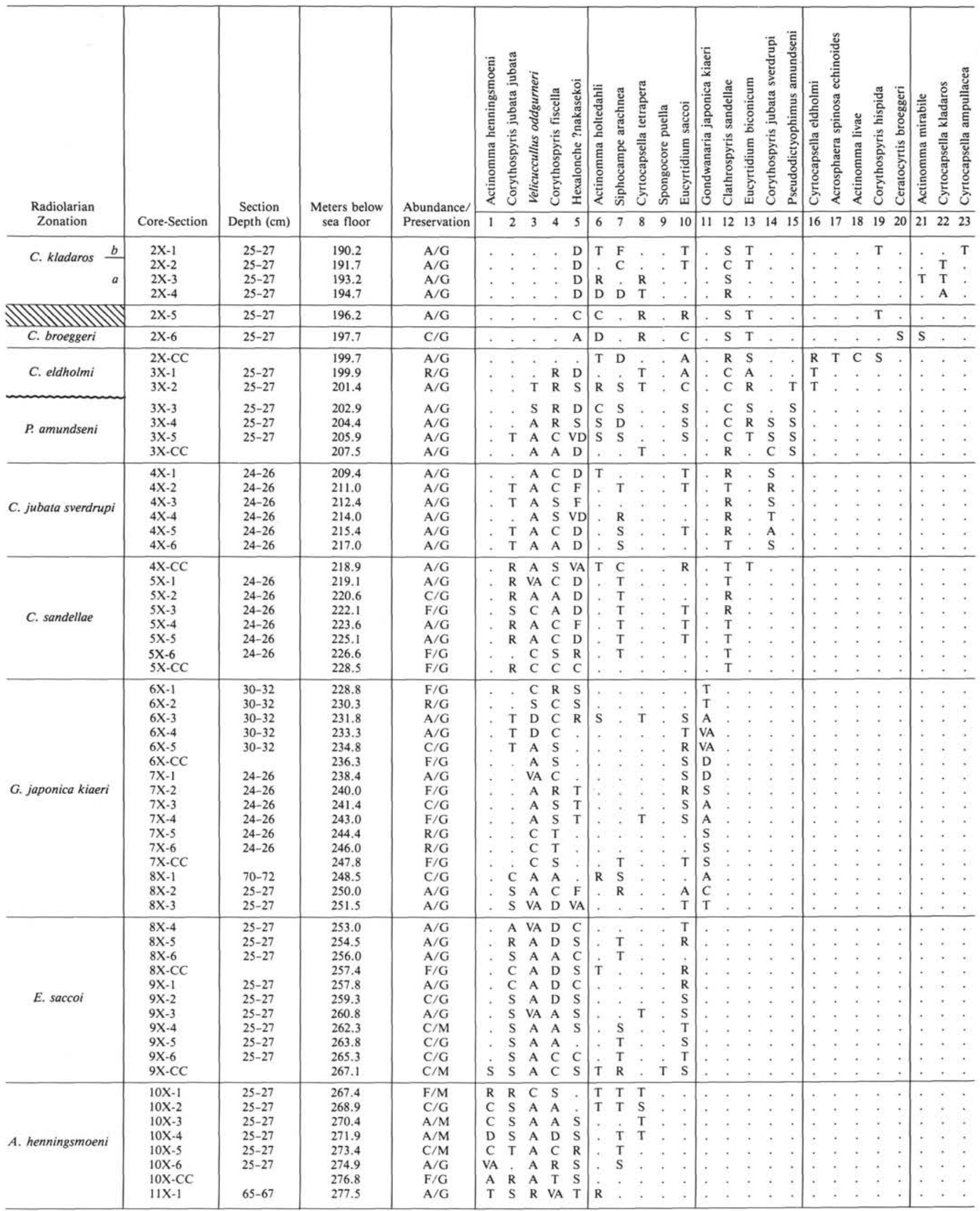


The major hiatus identified in Core 104-642D-3X is also present in Core 104-642B-23H. Sediments in Sections 104-642B-23H-1 to $-23 \mathrm{H}-3$ are assigned to the $C$. eldholmi Zone. Sedimentation older than the $C$. eldholmi Zone is more completely represented at Site 643 than at Site 642. As in Hole 642D the C. broeggeri Zone is very short, and its top must be truncated by an unconformity. C. stoermeri has been observed in both Holes 642B and $642 \mathrm{C}$, however, and we infer that this interval represents the basal-most part of the $C$. broeggeri Zone, which is not preserved in Hole 643A. The biostratigraphic effect of these two closelyspaced hiatuses is clearly evident in Cores 104-642B-22H and $23 \mathrm{H}$ (Table 2).

The C. kladaros and Pseudodictyophimus horrida Zones are more complete at Site 642, however, and Interzone B of Sections $104-642 \mathrm{~B}-19 \mathrm{H}-6$ to $19 \mathrm{H}-\mathrm{CC}$ and $104-642 \mathrm{C}-20 \mathrm{H}-3$ to $-20 \mathrm{H}-6$ was not recovered in Hole 643A. Above this interzone, there is a prominent change in the radiolarian fauna, which is marked by the first occurrence of Eucoronis fridtjofnanseni and is coincident with the first consistent preservation of calcareous microfossils. Because we regard $E$. fridtjofnanseni as an endemic or arctic species, the horizon of its abrupt first appearance in Holes $642 \mathrm{~B}$ and $642 \mathrm{C}$ is interpreted as an unconformity. Hence, we believe the sediments recording its early evolutionary history are missing at Site 642 .

A major unconformity has been interpreted as occurring in Sections 104-642B-19H-2 and 104-642C-19H-6 (Goll, this volume) on the basis of other biostratigraphic criteria, and the missing sediments have been regarded to range in age from 10.2 to $13.4 \mathrm{Ma}$. There is no evidence for this hiatus in the radiolarian succession, however, and the first appearance of $E$. fridtjofnanseni represents a more pronounced transition. Moreover, no reworked older radiolarians have been observed in the lower part of Core 104-642B-18H and upper Core 104-642B-19 or in mid to upper Core 104-642C-19H, as has been inferred from calcareous nannofossil evidence. These sediments are thought to represent a lag deposit (Goll, this volume).

The intervals $104-642 \mathrm{~B}-19 \mathrm{H}-2$ to $-15 \mathrm{H}-2$ (34.4 m thick) and $104-642 \mathrm{C}-19 \mathrm{H}-6$ to $16 \mathrm{H}-1$ (42.9 m thick) appear to represent relatively complete successions ranging from the upper $E$. fridtjofnanseni Zone to the lower Hexalonche esmarki Zone. The $H$. esmarki Zone is much thicker at Site 643, however, and a hiatus is regarded as the explanation for the absence of the majority of the $H$. esmarki Zone at Site 642 .

The intervals 104-642B-15H-1 to $-9 \mathrm{H}-1$ (56.6 m thick) and $104-642 \mathrm{C}-15 \mathrm{H}-6$ to $-9 \mathrm{H}-\mathrm{CC}$ (55.3 m thick) are regarded as relatively complete successions of the Tessarastrum thiedei Zone to Pseudodictyophimius gracilipes tetracanithus Zone, and they are the only representative of this sequence in Leg 104 recovery. The top of the Liriospyris cricus Zone in Holes 642B and 642C is located near the base of Chron $\mathrm{C} 3$ as interpreted by Bleil (this volume). The base of the $P$. gracilipes tetracanthus Zone in Hole 642B closely coincides with the top of Chron C3-N2, and can be said to have an age of $4.1 \mathrm{Ma}$. The very short interval 104-642C$15 \mathrm{H}-7$ to $-15 \mathrm{H}-\mathrm{CC}$ is older than the first occurrence of $T$. thiedei, and it is assigned to the Larcospira bulbosa Zone, which has not been detected in Hole 642B. The sediments below Sections 104$642 \mathrm{~B}-8 \mathrm{H}-\mathrm{CC}$ and 104-642C-9H-CC are regarded as older than the oldest sediments in Hole 644A, because of the absence of the Spongaster ?tetras Zone at Site 642 . The youngest $P$. gracilipes tetracanthus Zone sediments below the barren interval correlate with the reversed interval between Chron $\mathrm{C} 2 \mathrm{~A}-\mathrm{N} 2$ and $\mathrm{C} 2 \mathrm{~A}-$ N3 and have an approximate age of $3.1 \mathrm{Ma}$. The youngest $P$. gracilipes tetracanthus Zone sediments in Hole $642 \mathrm{C}$ are slightly older.

\section{Site 643}

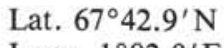

Long. $1^{\circ} 02.0^{\prime} \mathrm{E}$
Water depth: $2753 \mathrm{~m}$

Hole $643 \mathrm{~A}$ was continuously cored to a total depth of 565.2 mbsf. The lower $281.7 \mathrm{~m}$ of this hole penetrated compactionlaminated mudstones containing sparse lithified chalk beds (lithologic units IV-V; Cores 104-643A-31X-62X). Traces of sponge spicules and diatoms have been observed throughout Unit IV. Rare and poor- to moderately-preserved radiolarians occur in Cores 104-643A-31X and -32X. These faunas are dominated by long-ranging Corythospyris species, and we have not attempted to treat this recovery biostratigraphically. The restricted diversity of the radiolarian assemblage and the presence of more numerous sponge spicules suggests that the depositional environment may have been rather shallow and distant from open ocean environments where contemporaneous diverse radiolarian faunas probably flourished.

Core $104-643 \mathrm{~A}-30 \mathrm{X}$ is a transition interval for the radiolaria, which are more abundant and significantly more diverse in the diatomaceous mud of this core. This core is assigned to the Gondwanaria japonica kiaeri Zone.

Core 104-643A-29X represents the base of a siliceous ooze sequence $159.4 \mathrm{~m}$ thick, in which clay concentrations are generally less than $20 \%$. Siliceous microfossils, particularly diatoms, are significantly more abundant in this interval than in coeval sediments at Site 642 . The quality of radiolarian preservation is good throughout this interval, but frequencies of occurrence in our processed residues range from rare to abundant, depending on the diluting influence of other siliceous microfossils and volcanic glass.

The frequency and quality of preservation of radiolaria remains essentially unchanged in the overlying more clay-rich and calcareous interval (93.9-114.3 mbsf), but preservation quality degrades to only moderate or poor at shallower depths. Radiolarians are abundant in some of our processed residues from this upper interval, apparently because other siliceous microfossils have suffered a higher degree of dissolution loss. Radiolarians are increasingly less frequent with decreasing depth in Core $104-643 \mathrm{~A}-8 \mathrm{H}$, and they are essentially absent in Cores 104$643 \mathrm{~A}-7 \mathrm{H}$ to $-1 \mathrm{H}$.

The stratigraphic ranges of 41 taxa have been documented in 141 samples from Hole 643A (Table 5). The recovery from Cores $104-643 \mathrm{~A}-8 \mathrm{H}$ to $-30 \mathrm{X}$ ranges from the $G$. japonica kiaeri Zone to questionably the Tessarastrum thiedei Zone. As at Site 642, G. japonica kiaeri appears in Hole 643A recovery in an advanced stage of morphological development, and there is no indication of its early evolutionary history. The base of the $G$. japonica kiaeri Zone in Section 104-643A-30X-CC is probably coincident with an unconformity, and these oldest siliceous oozes are regarded as approximately coeval with Section 104-642D$8 \mathrm{X}-3$. The interval from Sections 104-643A-21X-1 to 30X-CC represents the same sequence (Pseudodicryophimus amundseni to G. japonica kiaeri Zones) encountered in Holes 642B and 642D. The Clathrospyris sandellae and $P$. amundseni Zones are significantly thicker in Hole 643A, however. The overlying 65.8 $\mathrm{m}$ of section is characterized by Interzone A and four partialrange zones (Spongotrochus vitabilis, Cycladophora davisiana cornutoides, Ceratocyrtis manumi, and Ceratocyrtis broeggeri). The coeval interval in Holes $642 \mathrm{~B}$ and $642 \mathrm{C}$ is represented by only $1.5 \mathrm{~m}$ of $C$. broeggeri Zone sediments. On the other hand, the $C$. broeggeri Zone at Site 642 is regarded as slightly older than the equivalent zone in Hole 643A, and the short Cyrtocapsella eldholmi Zone of Site 642 is missing at Site 643. Consequently, we infer a short hiatus at the base of the $C$. broeggeri Zone in Core 104-643A-15H.

The interval 101.4-120.4 mbsf ( $19 \mathrm{~m}$ thick) is interrupted by three short hiatuses. The short $C$. kladaros Subzone (Sections $104-643 \mathrm{~A}-13 \mathrm{H}-4$ to $-13 \mathrm{H}-\mathrm{CC}$ ) is immediately overlain by the $P$. horrida Zone, and the $C$. ampullacea Subzone and the Actinomma plasticum Zone are missing above this interval. Inter- 
zone B of Site 642 is also missing in Hole 643A, and the association of numerous Hexalonche ?nakasekoi in the $P$. horrida Zone of Hole 643A suggests that the upper part of this zone (where $H$. ?nakasekoi is rare or absent at Site 642) is missing at Site 643. The Clathrospyris vogti Zone at the top of this interval has not been observed in other Leg 104 recovery. The major unconformity interpreted to occur in Sections 104-642B-19H-2 and $642 \mathrm{C}-19 \mathrm{H}-5$ is also evident between Cores 104-643A-11H and $-12 \mathrm{H}$. The presence of a hiatus at this position is evidenced by the absence of the Eucoronis fridtjofnanseni Zone.

The interval $62.5-100.3$ mbsf ( $37.8 \mathrm{~m}$ thick) includes the youngest biosiliceous sediments in Hole $643 \mathrm{~A}$, and probably represents a continuous succession from the $E$. fridtjofnanseni/ Corythospyris reuschi Zone to the base of the Hexalonche esmarki Zone. The co-occurrence of calcareous microfossils and the existence of a reasonably interpretable paleomagnetic record permit confident age assignments for these sediments. The base of the $C$. reuschi Zone is coincident with the top of Chron C4AN2 (8.4 Ma). The base of the Spongurus cauleti Zone occurs in the lower part of Chron C4A-N1 and has a probable age of 8.1 $\mathrm{Ma}$. The base of the $H$. esmarki Zone occurs in the lowest reversed interval of Chron CA and has a probable age of $7.7 \mathrm{Ma}$. The top of the $H$. esmarki Zone probably occurs near the base of Chron C3A, but a hiatus and inconclusive polarity record preclude a confident age assignment. The $L$. bulbosa Zone has not been detected in Hole 643A. The sediment in Sections 104$643 \mathrm{~A}-8 \mathrm{H}-1$ to $8 \mathrm{H}-5$ is difficult to interpret biostratigraphically, because of poor preservation quality and the unusual faunal association. Trace occurrences of Phormospyris thespios suggest that this interval should occupy the base of the $T$. thiedei Zone, but $T$. thiedei has only one rare occurrence in Section 643A$8 \mathrm{H}-4$. The presence of Phorticium clevei indicates a position close to the top of this zone (just preceeding the first occurrences of Liriospyris cricus and Antarctissa whitei). We conclude that Core 104-643A-8H represents a highly condensed sedimentary unit, probably assignable to the $T$. thiedei Zone.

\section{Site 644}

\section{Lat. $66^{\circ} 40.7^{\prime} \mathrm{N}$}

Long. $4^{\circ} 34.6^{\prime} \mathrm{E}$

Water depth: $1227 \mathrm{~m}$

Two holes (644A and 644B) were continuously cored at this site to total depths of 252.8 and $127.7 \mathrm{mbsf}$, respectively. An excellent polarity reversal record and calcareous microfossil control indicate that Hole $644 \mathrm{~A}$ contains a reasonably complete record of sedimentation at this site for the past 2.9 m.y. Siliceous microfossils are preserved in younger sediments at Site 644 than at Sites 642 or 643 . Radiolarians are common to abundant and moderate- to well-preserved in Cores $104-644 \mathrm{~A}-31 \mathrm{H}$ to $-34 \mathrm{H}$, but only sporadic occurrences of moderate- to poorly preserved radiolarians are found in the glacial- interglacial marine sediments of Cores $104-644 \mathrm{~A}-11 \mathrm{H}$ to $-30 \mathrm{H}$. Radiolarians are essentially absent in Cores $104-644 \mathrm{~A}-1 \mathrm{H}$ to $-10 \mathrm{H}$. The youngest radiolarians observed in Hole $644 \mathrm{~A}$ have an age of approximately $0.75 \mathrm{Ma}$ (approximately coincident with the base of Chron C1N1). In anticipation of finding additional thin radiolarian-bearing horizons that had not been previously detected, over 600 smear slides of bulk sediments from Cores 104-644A-1H to $20 \mathrm{H}$ were examined, but only scattered trace occurrences were found. Variable concentrations of sponge spicules were the most common siliceous microfossils observed.

The stratigraphic ranges of 10 radiolarians are documented in 28 samples from Site 644 recovery (Table 6). Cores 104-644A$19 \mathrm{H}$ to $-34 \mathrm{H}$ have been examined at the rate of one sample per section. Only core-catcher samples were examined for Cores $104-644 \mathrm{~A}-11 \mathrm{H}$ to $-18 \mathrm{H}$. Samples from the intervals including Cores $104-644 \mathrm{~A}-14 \mathrm{H}$ to $-18 \mathrm{H},-23 \mathrm{H}-3$ to $-29 \mathrm{H}$, and Sections
$-30 \mathrm{H}-4$ to $-31 \mathrm{H}-2$ are barren of radiolarians or have only trace occurrences, and these intervals are not included in Table 7.

The Spongaster ?tetras Zone and Cycladophora davisiana davisiana Zone of Site 644 do not occur at Sites 642 or 643. The top of the $S$. ?tetras Zone is approximately coincident with the boundary between lithologic Units IC and II and occurs near the top of Chron C2A. Lophospyris pentagona quadriforis and Antarctissa whitei do not range above the S. ?tetras Zone. Although Hexacontium pachydermum is an extant species in the Norwegian Sea, it was not observed above the $S$. ?tetras Zone. What appear to be the immature skeletal growth stages of indeterminant Spumellaria are dominant in many of these samples and may indicate water mass conditions marginal for the survival of these species.

\section{BIOSTRATIGRAPHY}

The complete system of 28 zonal units proposed here is not represented in the recovery from any single Leg 104 site, because of hiatuses, non- overlapping sequences, or possible paleozoogeographic provincialism. Consequently, it has been necessary to reconstruct a composite section based on selected intervals from all the holes. Nevertheless, this radiolarian biozonation for the past 22 m.y. of pelagic sedimentation in the Norwegian Sea is not complete. A 3.2-m.y. hiatus has been proposed for the upper middle Miocene of Site 642, and this gap is even larger in Hole 643A (Goll, this volume). Additionally, there are smaller hiatuses present at both Sites 642 and 643 at the top of the Hexalonche esmarki Zone, and at the bases of the Eucoronis fridtjofnanseni Zone and Gondwanaria japonica kiaeri Zone. These hiatuses may have regional scope. There are probably other small unconformities that we have not been able to detect because comparative material is lacking.

Some intervals within the Neogene composite section are very difficult to characterize. Two short intervals (Sections 104643A-20X-6 to 20X-CC and 104-642B-19H-6 to $19 \mathrm{H}-\mathrm{CC}$ ) are designated as Interzones A and B, respectively, because no event markers were found to denote them. The Cyrtocapsella kladaros Zone is another interval that has been difficult to treat biostratigraphically. A long-ranging species with an event near the base of this interval has not been found, and it was necessary to construct a system of two subzones based on very short-ranging species: Cyrtocapsella kladaros and Cyrtocapsella ampullacea.

Because of problematical polarity reversal records and the essential absence of calcareous microfossils in sediments below Core 104-643A-11H and Sections 104-642B-19H-5/104-642C$20 \mathrm{H}-2$, age assignments in the early middle and early Miocene are based almost exclusively on siliceous microfossils. Radiolarians have been of only limited value in this endeavor because of temperate- to cold-water provincialism. Index taxa for the tropical Neogene radiolarian zonation are either absent in Leg 104 sediments or have only trace and highly sporadic ranges that severely degrade their biostratigraphic value. We found few Norwegian Sea species whose ranges have been documented in other North Atlantic localities, and we have been hampered in this effort by the lack of comparative material for examination. Nevertheless, there are some important exceptions. A few faunal similarities between lower Miocene sediments from the central equatorial Pacific and the Norwegian Sea have been detected. Correlations over such a vast geographic distance and $65^{\circ}$ of latitude are certainly hazardous and have been accepted only as a last resort. However, these observations help to place Leg 104 recovery in approximate chronologic position.

The biostratigraphic sequence from the Actinomma henningsmoeni Zone to the Pseudodictyophimus amundseni Zone appears to reflect temperate oceanic conditions in Leg 104 recovery. A. henningsmoeni has been observed in the mid Cyrtocapsella tetrapera Zone of DSDP Hole 77B, and consequently the 
Table 5. Stratigraphic range chart for radiolaria in Hole 643A.

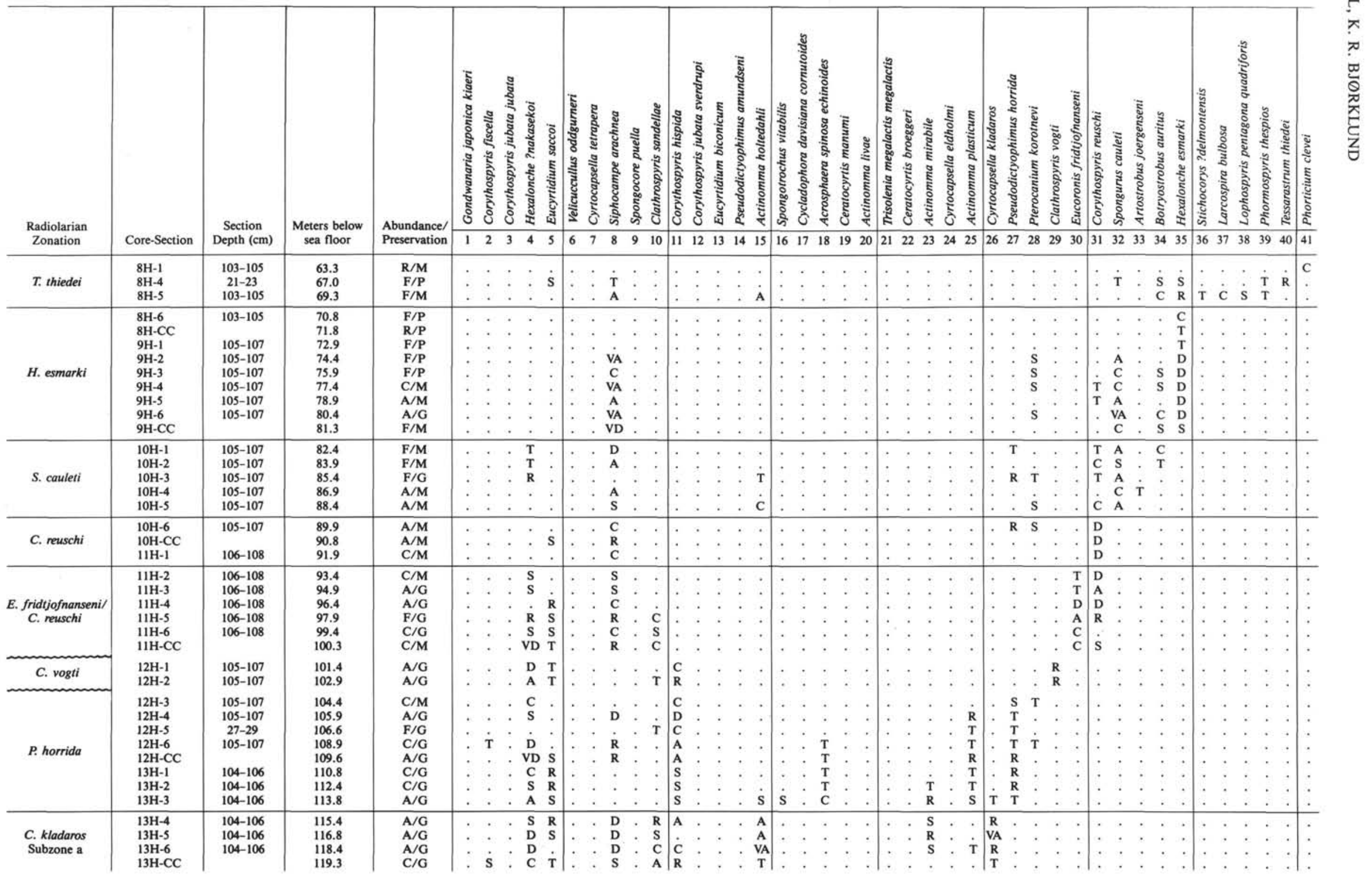




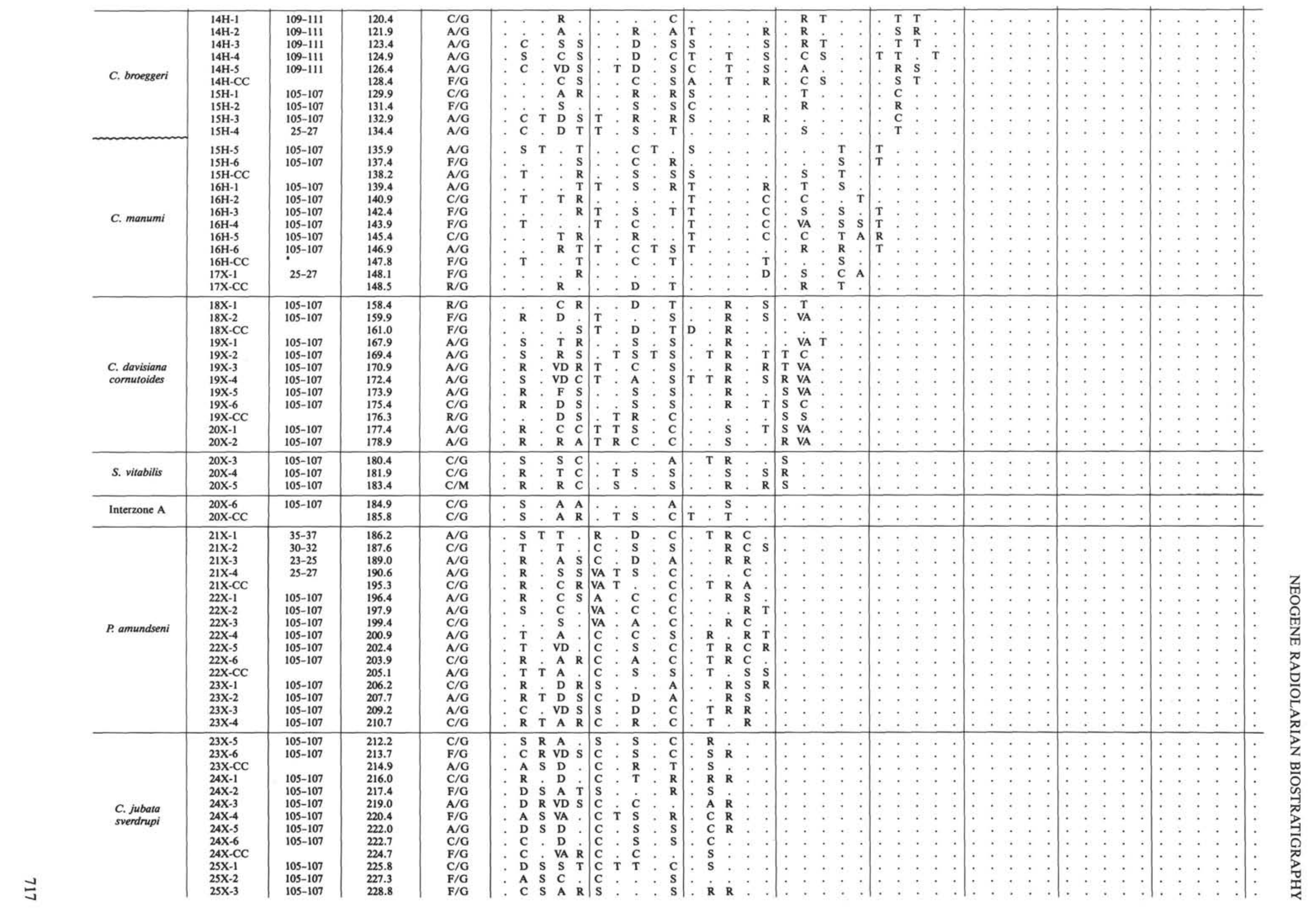


Table 5 (continued).

\begin{tabular}{|c|c|c|c|c|c|c|c|c|c|c|c|c|c|c|c|c|c|c|c|c|c|c|c|c|c|c|c|c|c|c|c|c|c|c|c|c|c|}
\hline \multirow{2}{*}{$\begin{array}{l}\text { Radiolarian } \\
\text { Zonation }\end{array}$} & \multirow[b]{2}{*}{ Core-Section } & \multirow{2}{*}{$\begin{array}{c}\text { Section } \\
\text { Depth (cm) }\end{array}$} & \multirow{2}{*}{$\begin{array}{l}\text { Meters below } \\
\text { sea floor }\end{array}$} & \multirow[t]{2}{*}{$\mid$} & \multicolumn{4}{|c|}{ 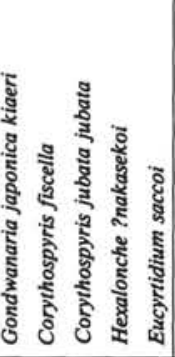 } & \multicolumn{4}{|c|}{ 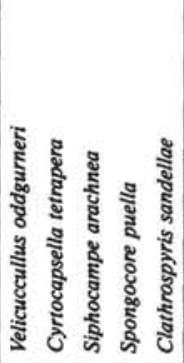 } & \multicolumn{5}{|c|}{ 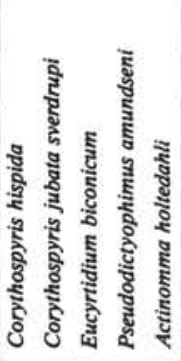 } & \multicolumn{4}{|c|}{ 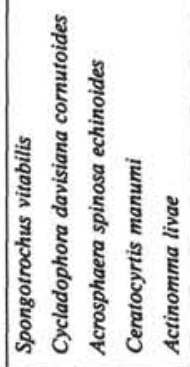 } & \multicolumn{4}{|c|}{ 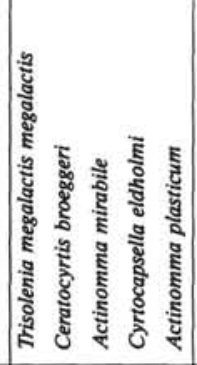 } & \multicolumn{3}{|c|}{ 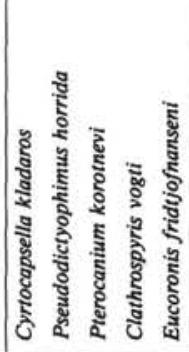 } & \multicolumn{4}{|c|}{ 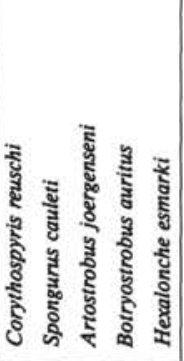 } & \multicolumn{5}{|c|}{ 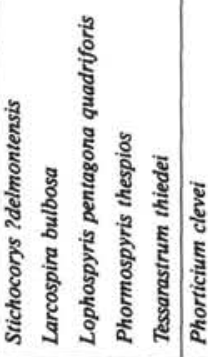 } \\
\hline & & & & & 1 & 23 & 34 & 45 & 6 & 7 & 8 & 910 & & 112 & 13 & 141 & \begin{tabular}{l|l}
15 & 1 \\
\end{tabular} & $16 \quad 17$ & 18 & 19 & $2022-3 x-2$ & 2122 & 2223 & 242 & \begin{tabular}{l|l}
25 & 2 \\
-1
\end{tabular} & $26 \quad 27$ & 2829 & 2930 & 31 & 3233 & $33 \quad 34$ & 435 & 363 & 3738 & $38 \quad 39$ & 940 & 41 \\
\hline C. sandellae & $\begin{array}{l}25 \mathrm{X}-4 \\
25 \mathrm{X}-5 \\
25 \mathrm{X}-6 \\
25 \mathrm{X}-\mathrm{CC} \\
26 \mathrm{X}-1 \\
26 \mathrm{X}-2 \\
26 \mathrm{X}-3 \\
26 \mathrm{X}-4 \\
26 \mathrm{X}-5 \\
26 \mathrm{X}-6 \\
26 \mathrm{X}-\mathrm{CC} \\
27 \mathrm{X}-1 \\
27 \mathrm{X}-2 \\
27 \mathrm{X}-3 \\
27 \mathrm{X}-4 \\
27 \mathrm{X}-5 \\
27 \mathrm{X}-6 \\
27 \mathrm{X}-\mathrm{CC}\end{array}$ & $\begin{array}{l}105-107 \\
105-107 \\
105-107 \\
105-107 \\
105-107 \\
105-107 \\
105-107 \\
105-107 \\
105-107 \\
\\
105-107 \\
105-107 \\
105-107 \\
105-107 \\
105-107 \\
105-107\end{array}$ & $\begin{array}{l}230.3 \\
231.8 \\
233.3 \\
234.7 \\
235.8 \\
237.3 \\
238.8 \\
240.3 \\
241.8 \\
243.3 \\
244.3 \\
245.4 \\
246.9 \\
248.4 \\
249.9 \\
251.4 \\
252.9 \\
254.0\end{array}$ & $\begin{array}{l}\text { F/G } \\
\text { F/G } \\
\text { C/G } \\
\text { R/G } \\
R / G \\
R / G \\
R / G \\
R / G \\
\text { C/G } \\
\text { /G } \\
\text { C/G } \\
\text { C/G } \\
\text { C/G } \\
\text { C/G } \\
\text { C/G } \\
\text { C/G } \\
\text { C/G } \\
\text { F/G }\end{array}$ & $\begin{array}{l}: \\
\vdots \\
\vdots \\
\vdots \\
\text { s } \\
\text { s } \\
\vdots \\
\vdots \\
\vdots\end{array}$ & $\begin{array}{ll}\text { C } \\
\text { A } \\
\text { C } \\
\text { S } \\
\text { R } \\
\text { T } \\
\text { C } \\
\text { C } \\
\text { C } \\
\text { S } \\
\text { C } \\
\text { S } \\
\text { C } \\
\text { C } \\
\text { C } \\
\text { C } \\
\text { A } \\
\text { R } \\
\text { S }\end{array}$ & 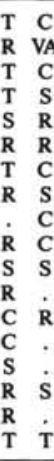 & 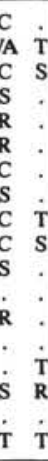 & $\begin{array}{l}\text { S } \\
\text { C } \\
\text { S } \\
\text { C } \\
\text { R } \\
\text { R } \\
\text { S } \\
\text { A } \\
\text { C } \\
\text { A } \\
\text { A } \\
\text { C } \\
\text { A } \\
\text { A } \\
\text { A } \\
\text { A } \\
\text { C } \\
\text { A }\end{array}$ & $\begin{array}{c}\text { T } \\
\text { T } \\
\vdots \\
\vdots \\
\vdots \\
\vdots \\
\vdots \\
\dot{T} \\
\vdots\end{array}$ & $\begin{array}{l}\dot{\mathrm{T}} \\
\mathrm{S} \\
\dot{0} \\
\vdots \\
\dot{\mathrm{R}} \\
\dot{\mathrm{S}} \\
\vdots \\
\vdots \\
\vdots \\
\dot{\mathrm{R}} \\
\mathrm{C}\end{array}$ & $\begin{array}{ll}: & \mathrm{C} \\
: & \mathrm{S} \\
: & \mathrm{S} \\
: & \mathrm{T} \\
: & \mathrm{T} \\
: & \mathrm{T} \\
: & \mathrm{R} \\
: & \mathrm{T} \\
: & \mathrm{T} \\
: \mathrm{R} \\
: & \mathrm{S} \\
: & \mathrm{T} \\
: & \mathrm{T} \\
: & \mathrm{T} \\
\end{array}$ & & 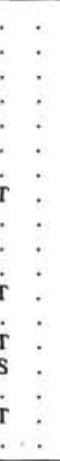 & $\begin{array}{l}: \\
\vdots \\
\vdots \\
\vdots \\
\vdots \\
:\end{array}$ & $\begin{array}{l}: \\
: \\
: \\
: \\
: \\
: \\
: \\
: \\
:\end{array}$ & 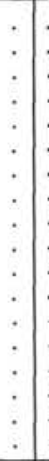 & $\begin{array}{l}\vdots \\
\vdots\end{array} \vdots$ & $\begin{array}{l}: \\
: \\
: \\
: \\
: \\
: \\
:\end{array}$ & $\begin{array}{l}: \\
\vdots \\
\vdots \\
\vdots \\
\vdots \\
\vdots \\
:\end{array}$ & $\begin{array}{l}: \\
\vdots \\
\vdots \\
\vdots \\
\vdots \\
\vdots \\
\vdots \\
\vdots \\
:\end{array}$ & $\begin{array}{l}: \\
\vdots \\
\vdots\end{array}$ & 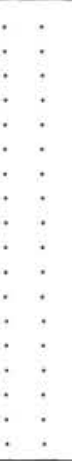 & $\begin{array}{l}\vdots \\
\vdots \\
\vdots\end{array}$ & & 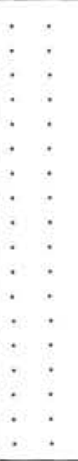 & $\begin{array}{l}: \\
\vdots \\
\vdots\end{array}$ & $\begin{array}{l}\vdots \\
\vdots \\
\vdots \\
\vdots\end{array}$ & $\begin{array}{l}\vdots \\
\vdots \\
\vdots \\
\vdots\end{array}$ & 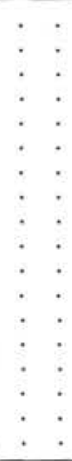 & $\begin{array}{l}0 \\
\vdots \\
\vdots \\
\vdots\end{array}$ & $\begin{array}{l}\vdots \\
\vdots \\
\vdots \\
\vdots \\
\vdots \\
\vdots \\
\vdots\end{array}$ & $\begin{array}{l}: \\
: \\
: \\
\vdots \\
\vdots \\
\vdots \\
\vdots \\
: \\
:\end{array}$ & $\begin{array}{l}: \\
\vdots \\
:\end{array}$ & $\begin{array}{l} \\
\vdots \\
\vdots\end{array}$ & $\begin{array}{l}: \\
\vdots \\
\vdots \\
\vdots \\
\vdots \\
\vdots \\
:\end{array}$ & \\
\hline G. japonica kiaeri & $\begin{array}{l}28 \mathrm{X}-1 \\
28 \mathrm{X}-2 \\
28 \mathrm{X}-3 \\
28 \mathrm{X}-4 \\
28 \mathrm{X}-5 \\
28 \mathrm{X}-6 \\
28 \mathrm{X}-\mathrm{CC} \\
29 \mathrm{X}-1 \\
29 \mathrm{X}-2 \\
29 \mathrm{X}-3 \\
29 \mathrm{X}-4 \\
29 \mathrm{X}-5 \\
29 \mathrm{X}-6 \\
29 \mathrm{X}-\mathrm{CC} \\
30 \mathrm{X}-\mathrm{CC}\end{array}$ & $\begin{array}{l}105-107 \\
105-107 \\
105-107 \\
105-107 \\
105-107 \\
105-107\end{array}$ & $\begin{array}{l}255.2 \\
256.7 \\
258.2 \\
259.7 \\
261.2 \\
262.7 \\
263.9 \\
265.9 \\
266.5 \\
266.5 \\
268.0 \\
271.5 \\
271.0 \\
273.5 \\
283.7 \\
283.5\end{array}$ & $\begin{array}{l}\text { C/G } \\
\text { F/G } \\
\text { A/G } \\
\text { F/G } \\
\text { C/G } \\
\text { A/G } \\
\text { F/G } \\
\text { A/G } \\
\text { C/G } \\
\text { A/G } \\
\text { A/G } \\
\text { A/G } \\
\text { A/G } \\
\text { C/G } \\
\text { F/M }\end{array}$ & $\begin{array}{l}R \\
\text { R } \\
\text { A } \\
\text { C } \\
\text { S } \\
\text { A } \\
\text { C } \\
\text { S } \\
\text { S } \\
\text { VA } \\
\text { VA } \\
\text { VA } \\
\text { VA } \\
\text { A } \\
\text { R }\end{array}$ & $\begin{array}{ll}\text { A } & \text { I } \\
\text { C } & \\
\text { A } & \\
\text { S } & \\
\text { S } \\
\text { A } \\
\text { R } \\
\text { S } \\
\text { C } \\
\text { A } \\
\text { D } \\
\text { C } \\
\text { D } \\
\text { C } \\
\text { T } \\
\end{array}$ & $\begin{array}{ll}R & C \\
\text { R } & \text { A } \\
\text { R } & \text { S } \\
\text { R } & \text { R } \\
\text { R } & \text { R } \\
\text { T } & \text { R } \\
\text { P } & \text { T } \\
\text { R } & \text { S } \\
\text { S } & \text { R } \\
\text { S } & \text { A } \\
\text { S } & \text { S } \\
\text { C } & \text { C } \\
\text { S } & \text { S } \\
\text {. } & \text {. }\end{array}$ & $\begin{array}{ll}C & \text { C } \\
\text { A } & \text { D } \\
\text { S } & \text { A } \\
\text { R } & \text { S } \\
\text { R } & \text { T } \\
R & \text { C } \\
\text { T } & \text { R } \\
\text { T } & \text { S } \\
\text { S } & \text { S } \\
\text { A } & \text { R } \\
\text { A } & \text { S } \\
\text { S } & \text { C } \\
\text { C } & \text { A } \\
\text { S } & \text { C } \\
\end{array}$ & $\begin{array}{l}\mathrm{A} \\
\mathrm{C} \\
\mathrm{S} \\
\mathrm{R} \\
\mathrm{R} \\
\mathrm{R} \\
\text { : } \\
\mathrm{R} \\
\mathrm{R} \\
\mathrm{R} \\
\mathrm{R} \\
\mathrm{R} \\
\end{array}$ & $\begin{array}{l}\mathrm{T} \\
\vdots \\
\vdots \\
\vdots \\
\vdots \\
\dot{\mathrm{T}} \\
\mathrm{T} \\
\vdots\end{array}$ & $\begin{array}{l}\mathrm{T} \\
\mathrm{C} \\
\mathrm{A} \\
\mathrm{T} \\
\mathrm{S} \\
\mathrm{S} \\
\vdots \\
\vdots \\
\vdots \\
\vdots \\
\vdots\end{array}$ & $\begin{array}{l}\vdots \\
\mathrm{T}\end{array}$ & & $\begin{array}{l}0: \\
\vdots \\
\vdots\end{array}:$ & 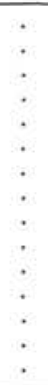 & $:$ & 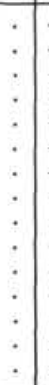 & 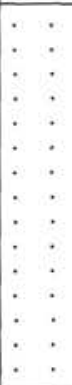 & $\begin{array}{l}: \\
: \\
: \\
: \\
: \\
:\end{array}$ & : & $\begin{array}{l}: \\
\vdots \\
\vdots \\
: \\
\vdots \\
: \\
: \\
:\end{array}$ & $\begin{array}{l:}: \\
:\end{array}$ & $: \vdots:$ & $\begin{array}{l}: \\
: \\
: \\
\vdots \\
: \\
: \\
:\end{array}$ & $\begin{array}{l}: \\
\vdots \\
\vdots \\
: \\
:\end{array}$ & $\begin{array}{l}: \\
\vdots \\
\vdots\end{array}:$ & $\begin{array}{l}: \\
\vdots \\
\vdots\end{array}$ & $\begin{array}{l} \\
\vdots \\
\vdots\end{array}$ & $\begin{array}{l}: \\
\vdots \\
\vdots\end{array}$ & $\begin{array}{l}: \\
\vdots \\
\vdots \\
\vdots\end{array}$ & $\vdots:$ & $\begin{array}{l} \\
\vdots \\
\vdots\end{array}:$ & $\begin{array}{l}: \\
\vdots \\
\vdots \\
\vdots \\
\vdots \\
\vdots \\
:\end{array}$ & $\begin{array}{l}: \\
\vdots \\
\vdots\end{array}$ & $\begin{array}{l}: \\
\vdots \\
\vdots\end{array}$ & 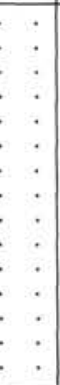 & \\
\hline
\end{tabular}


Table 6. Stratigraphic range chart for radiolaria in Hole 644A.

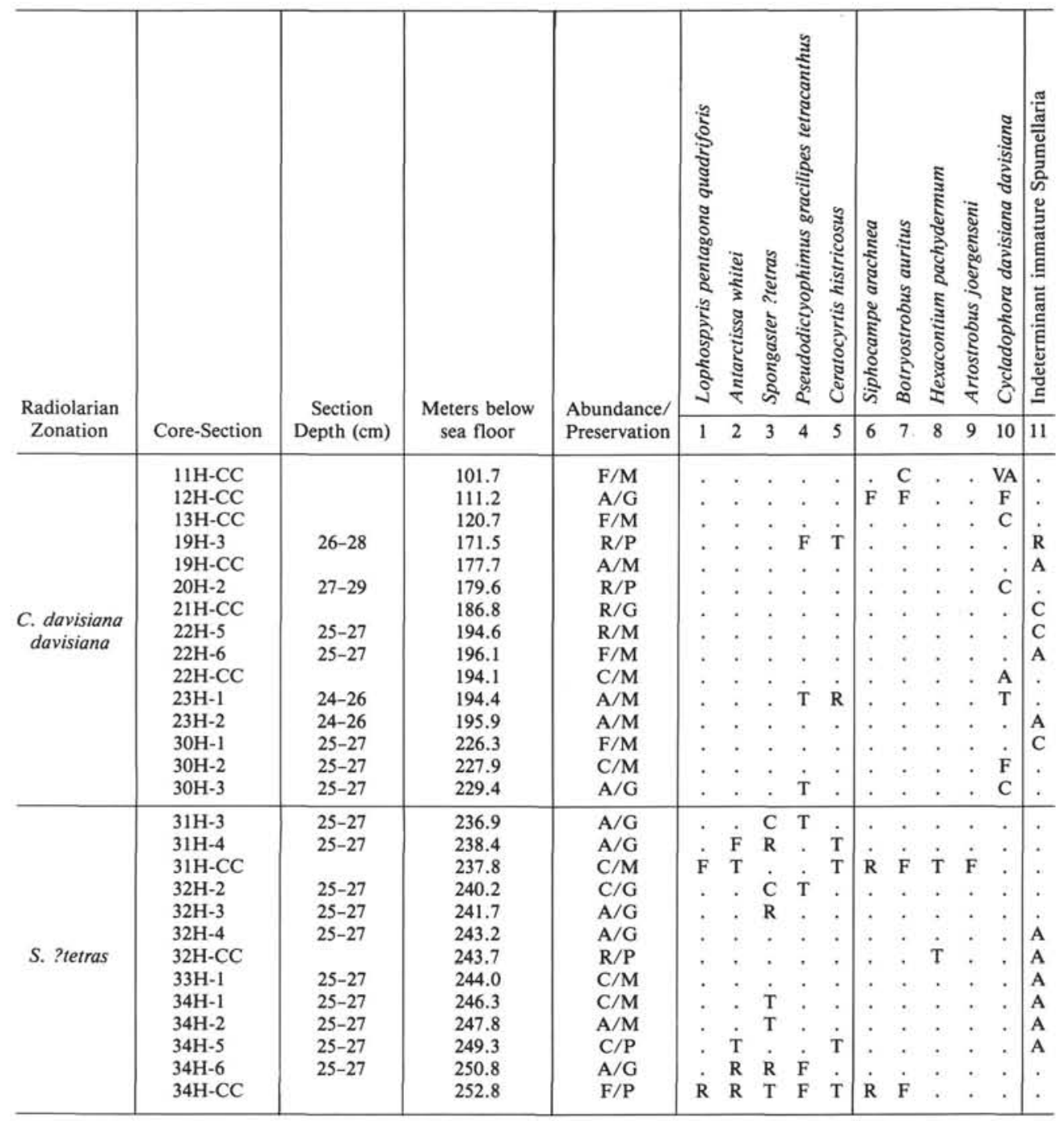

age of the $A$. henningsmoeni Zone of Hole $642 \mathrm{D}$ is regarded as approximately $22 \mathrm{Ma}$. The origination of Eucyrtidium saccoi in the overlying section of Hole 642D is believed to be the result of local evolution, probably influenced by temperate water input into the Norwegian Sea. We employ E. saccoi as the name for a broad range of similar morphologies, which will probably require more refined taxonomic treatment. Above the $E$. saccoi Zone in Hole 642D (Table 4, Col. 10), this species complex has sporadic occurrences in low frequencies at both Sites 642 and 643 . Its youngest recorded occurrence is in the $P$. gracilipes tetracanthus Zone of Hole 642B (Table 2, Col. 7). The species is not recorded above the base of the $E$. fridtjofnanseni Zone of Hole 642C (Table 3, Col. 3), however. Gondwanaria japonica kiaeri has abrupt first occurrences in Holes 642D and 643A associated with an interpreted hiatus, but its probable precursor has a long prior history in Norwegian Sea sediments. Undescribed species in the lower G. japonica kiaeri Zone have also been observed in the upper Stichocorys delmontensis Zone to lower Stichocorys wolfii Zone of DSDP Hole 77B, and the age of these sediments is regarded as approximately $19 \mathrm{Ma}$. In Hole $643 \mathrm{~A}$, Velicuccullus oddgurneri is absent from the base of the $G$. japonica kiaeri Zone, whereas the species is continuously present in this zone in Hole 642D. These associations suggest that the bases of the G. japonica kiaeri Zone are not exactly coeval at these two sites. $V$. oddgurneri is consistently present in the overlying sediments of Hole $643 \mathrm{~A}$ with frequencies ranging from rare to very abundant. The species has its last true occurrence at the top of the $P$. amundseni Zone, although trace fragments persist to the $C$. broeggeri Zone (Table 2, Col. 6). V. oddgurneri has higher frequencies at Site 642 , with generally abundant to dominant occurrences and abrupt terminations at the top of the unconformity-bounded $P$. amundseni Zone in Holes 642D (Table 4, Col. 3) and 642B (Table 2, Col. 2). Clathrospyris sandellae has also been observed (but not reported) in Miocene sediments from the North Pacific. The species first occurs in Norwegian Sea sediments in very low frequencies, and there is no obvious local precursor. $C$. sandellae reaches frequencies of common to abundant in the uppermost C. jubata sverdrupi Zone of Hole 643A (Table 5, Col. 10) and is generally numerous up to the $C$. davisiania cornutoides Zone. The species is less abundant in coeval sediments of Site 642 (Table 2, Col. 6, and Table 4, Col. 12). C. sandellae undergoes two episodes of declining frequencies and sporadic occurrences in the $C$. manumi Zone of Site 643 and the $P$. horrida Zone of Sites 642 and 643 .

Corythospyris jubata sverdrupi appears to be the terminal morph of a lineage having a very long residence in the Norwegian Sea. Corythospyris taxa appear to have been continuous occupants of the Norwegian Sea since at least the late Eocene, although the youngest species disappeared in the $T$. thiedei Zone. 
Table 7. Distribution and boundary criteria for radiolaria zones in Leg 104 recovery. The age estimates are taken from Goll (this volume).

\begin{tabular}{|c|c|c|c|c|c|c|c|c|}
\hline \multirow{2}{*}{ 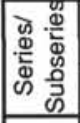 } & \multicolumn{5}{|c|}{ Leg 104 Holes } & \multirow{2}{*}{ Radiolaria Zone } & \multirow{3}{*}{ Boundary Criteria } & \multirow{3}{*}{ 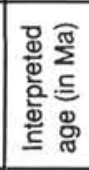 } \\
\hline & $642 B$ & $642 \mathrm{C}$ & $642 D$ & $643 A$ & $644 \mathrm{~A}$ & & & \\
\hline Pleist. & \multirow{2}{*}{ - } & \multirow{2}{*}{ - } & \multirow{2}{*}{ - } & \multirow{2}{*}{-} & \multirow{2}{*}{$\begin{array}{c}\text { Top to } \\
30-3,25-27 \mathrm{~cm} \\
0-229.4 \mathrm{mbsf} \\
\end{array}$} & Cyclaclophora davisiana & & \\
\hline \multirow{7}{*}{ 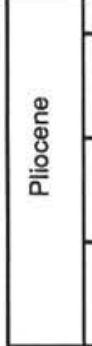 } & & & & & & Taxon range zone & \multirow{2}{*}{$\begin{array}{l}\text { irst occurrence of } \\
\text { Cycladophora } \\
\text { visiana davisiana }\end{array}$} & \multirow{2}{*}{2.6} \\
\hline & - & - & \multirow{2}{*}{-} & \multirow{2}{*}{-} & $\begin{array}{c}31-3,25-27 \mathrm{~cm} \\
\text { to } 34-\mathrm{cc}\end{array}$ & \multirow{2}{*}{$\begin{array}{l}\text { Spongaster? tetras } \\
\text { Taxon range zone }\end{array}$} & & \\
\hline & & & & & 236.9-252.8mbsf & & \multirow{2}{*}{$\begin{array}{l}\text { First occurrence of } \\
\text { Spongaster? tetras }\end{array}$} & \multirow{2}{*}{$\begin{array}{l}2.9- \\
3.1\end{array}$} \\
\hline & $\begin{array}{l}9-1,75-77 \mathrm{~cm} \text { to } \\
10-1,75-77 \mathrm{~cm}\end{array}$ & $\begin{array}{c}9-\mathrm{cc} \text { to } \\
11-3,70-72 \mathrm{~cm}\end{array}$ & \multirow[t]{2}{*}{-} & \multirow[t]{2}{*}{-} & \multirow[t]{2}{*}{-} & $\begin{array}{l}\text { Pseudodictyophimus } \\
\text { gracilipes tetracanthus }\end{array}$ & & \\
\hline & 67.2-76.7mbsf & $63.5-76.7 \mathrm{mbsf}$ & & & & Partial range zone & $\begin{array}{l}\text { st occurrence of } \\
P \text {. gracilipes }\end{array}$ & \multirow{2}{*}{4.0} \\
\hline & $\begin{array}{c}10-2,25-27 \mathrm{~cm} \\
\text { to } 11-6,25-27 \mathrm{~cm}\end{array}$ & $\begin{array}{c}11-4,70-72 \mathrm{~cm} \\
\text { to } 12-\mathrm{cc}\end{array}$ & \multirow{2}{*}{-} & \multirow{2}{*}{-} & \multirow{2}{*}{ - } & \multirow{2}{*}{$\begin{array}{l}\text { Antarctissa whitei } \\
\text { Interval zone }\end{array}$} & tetracanthus & \\
\hline & $77.7-93.2 \mathrm{mbsf}$ & $78.2-92.0 \mathrm{mbsf}$ & & & & & \multirow{2}{*}{$\begin{array}{l}\text { Last occurrence of } \\
\text { L. cricus }\end{array}$} & \multirow{2}{*}{5.2} \\
\hline \multirow{16}{*}{ 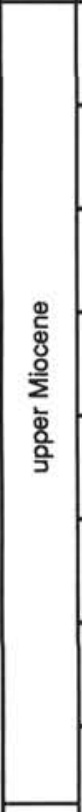 } & $\begin{array}{c}11-7,25-27 \mathrm{~cm} \\
\text { to } 12-\mathrm{cc}\end{array}$ & $\begin{array}{c}13-1,70-72 \mathrm{~cm} \text { to } \\
14-2,70-72 \mathrm{~cm}\end{array}$ & - & - & - & Liriospyris cricus & & \\
\hline & $94.7-104.2 \mathrm{mbst}$ & $92.7-103.7 \mathrm{mbsf}$ & 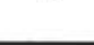 & 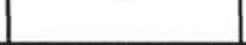 & 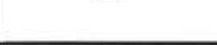 & & & 5.5 \\
\hline & $13-1,24-26 \mathrm{~cm}$ & $\begin{array}{c}14-3,70-72 \mathrm{~cm} \\
\end{array}$ & & $8-1,103-105 \mathrm{~cm}$ to & & Tessarastrum thiedei & L. cricus & 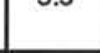 \\
\hline & $\begin{array}{l}\text { to } 15-1,27-29 \mathrm{~cm} \\
104.4-123.8 \mathrm{mbsf}\end{array}$ & $\begin{array}{l}\text { to } 15-6,25-2 / \mathrm{cm} \\
105.2-118.8 \mathrm{mbsf}\end{array}$ & - & $\begin{array}{r}8-5,103-105 \mathrm{~cm} \\
63.3-69.3 \mathrm{mbsf} \\
\end{array}$ & - & Partial range zone & occurrence of & 62 \\
\hline & - & $\begin{array}{c}15-7,25-27 \mathrm{~cm} \\
\text { to } 15-\mathrm{cc}\end{array}$ & - & - & - & Larcospira bulbosa & thiedei & 0.6 \\
\hline & - & $120.3-120.5 \mathrm{mbsf}$ & - & & & Partial range zone & cee of & 6.3- \\
\hline & $15-2,27-29 \mathrm{~cm}$ & $\begin{array}{l}16-1,25-27 \mathrm{~cm} \\
\text { to } 16-3,25-27 \mathrm{~cm}\end{array}$ & - & $\begin{array}{c}8-6,103-105 \mathrm{~cm} \\
\text { to } 9-\mathrm{cc}\end{array}$ & - & Hexalonche esmarki & ulbosa & 6.9 \\
\hline & $1253.3 \mathrm{mbsf}$ & $120.8-123.8 \mathrm{mbsf}$ & - & 70.8--81.3mbsf & & Partial range zone & it occurrence of & 7.7- \\
\hline & $\begin{array}{c}15-3,27-29 \mathrm{~cm} \text { to } \\
16-2,31-33 \mathrm{~cm}\end{array}$ & $\begin{array}{c}\begin{array}{c}16-4,25-27 \mathrm{~cm} \\
\text { to } 16-5,25-27 \mathrm{~cm}\end{array}\end{array}$ & - & \begin{tabular}{|c|}
$10-1,105-107 \mathrm{~cm}$ \\
to $10-5,105-107 \mathrm{~cm}$
\end{tabular} & - & Spongurus cauleti & esmarki & 7.9 \\
\hline & $126.8-129.9 \mathrm{mbsf}$ & $125.3-126.8 \mathrm{mbsf}$ & - & $82.4-88.4 \mathrm{mbsf}$ & 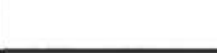 & Partial range zone & & 81 \\
\hline & $\begin{array}{l}16-3,31-33 \mathrm{~cm} \\
\text { to } 16-7,31-33 \mathrm{~cm}\end{array}$ & $\begin{array}{l}16-6,25-27 \mathrm{~cm} \\
\text { to } 17-2,25-27 \mathrm{~cm}\end{array}$ & - & \begin{tabular}{|c|}
$10-6,105-107 \mathrm{~cm}$ \\
to $11-1,106-108 \mathrm{~cm}$
\end{tabular} & - & $\begin{array}{l}\text { Corythospyris } \\
\text { reuschi }\end{array}$ & Spongurus cauleti & 8.1 \\
\hline & $131.4-137.4 \mathrm{mbsf}$ & $128.3-131.8 \mathrm{mbsf}$ & & 89.9-91.9mbsf & & Interval zone & $\begin{array}{l}\text { Last consistent } \\
\text { occurrence of }\end{array}$ & $8.3-8.6$ \\
\hline & $\begin{array}{c}16-\mathrm{cc} \text { to } \\
18-4,24-26 \mathrm{~cm}\end{array}$ & $\begin{array}{l}17-3,25-27 \mathrm{~cm} \\
\text { to } 19-2,25-27 \mathrm{~cm}\end{array}$ & - & $\begin{array}{c}11-2,106-108 \mathrm{~cm} \\
\text { to } 11-\mathrm{cc}\end{array}$ & - & E. fridtjofnanseni/C.reusch & E. fridtjofnanseni & \\
\hline & $138.0-152.5 \mathrm{mbsf}$ & 133.3-150.8mbsf & 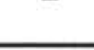 & $93.4-100.3 \mathrm{mbsf}$ & 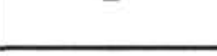 & range zone & $\begin{array}{l}\text { st occurrence of } \\
\text { Corythospyris }\end{array}$ & 9.5 \\
\hline & $\begin{array}{c}18-5,24-26 \mathrm{~cm} \text { to } \\
19-5,24-26 \mathrm{~cm}\end{array}$ & $\begin{array}{c}19-3,25-27 \mathrm{~cm} \\
\text { to } 20-2,25-27 \mathrm{~cm}\end{array}$ & - & - & - & Eucoronis fridtjofnanseni & reuschi & 9.0 \\
\hline & $154.0-163.9 \mathrm{mbsf}$ & $152.3-160.3 \mathrm{mbsf}$ & & & & & & $\sim 13.5$ \\
\hline & & & & $12-1,105-107 \mathrm{~cm}$ & - & Clathrospyris vogti & E. fridtjofnanseni & 13.6 \\
\hline ¿ & - & - & - & $\begin{array}{c}\text { to } 12-2,105-107 \mathrm{~cm} \\
101.4-102.9 \mathrm{mbsf}\end{array}$ & - & Taxon range zone & occurrence of & $\sim 13.7$ \\
\hline$\sum_{0}^{0}$ & $\begin{array}{c}19-6,24-26 \mathrm{~cm} \\
\text { to } 19-\mathrm{cc}\end{array}$ & $\begin{array}{c}20-3,25-27 \mathrm{~cm} \\
\text { to } 20-6,25-27 \mathrm{~cm}\end{array}$ & - & - & - & Interzone B & & 13.8 \\
\hline$\frac{\omega}{\overline{0}}$ & $165.4-167.2 \mathrm{mbsf}$ & $161.8-166.3 \mathrm{mbsf}$ & - & - & & & Last consistent & $\sim 141$ \\
\hline $\bar{\varepsilon}$ & $\begin{array}{c}20-1,24-26 \mathrm{~cm} \\
\text { to } 21.5,24-26 \mathrm{~cm}\end{array}$ & $\begin{array}{c}20-7,25-27 \mathrm{~cm} \\
\text { to } 22-3,25-27 \mathrm{~cm}\end{array}$ & - & \begin{tabular}{|c|}
$12-3,105-107 \mathrm{~cm}$ \\
to $13-3,105-107 \mathrm{~cm}$
\end{tabular} & - & $\begin{array}{l}\text { Pseudodictyophimus } \\
\text { horrida }\end{array}$ & $\begin{array}{l}\text { occurrence of } \\
\text { P. horrida }\end{array}$ & 9.14 .1 \\
\hline & $167.6-183.3 \mathrm{mbsf}$ & $167.8-180.8 \mathrm{mbsf}$ & & $104.4-113.8 \mathrm{mbsf}$ & & Taxon range zone & First occurrence of & $\sim 145$ \\
\hline
\end{tabular}




\begin{tabular}{|c|c|c|c|c|c|c|c|c|}
\hline \multirow{2}{*}{ 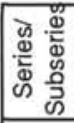 } & \multicolumn{5}{|c|}{ Leg 104 Holes } & \multirow{2}{*}{ Radiolaria Zone } & \multirow{3}{*}{ Boundary Criteria } & \multirow{3}{*}{ 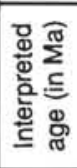 } \\
\hline & $642 B$ & $642 \mathrm{C}$ & $642 D$ & $643 A$ & $644 A$ & & & \\
\hline \multirow{12}{*}{ 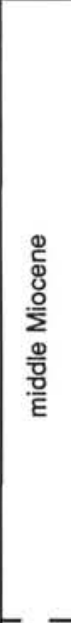 } & $\begin{array}{l}21-6,24-26 \mathrm{~cm} \\
\text { to } 22-1,75-77 \mathrm{~cm}\end{array}$ & $\begin{array}{l}22-4,25-27 \mathrm{~cm} \\
\text { to } 23-2,24-26 \mathrm{~cm}\end{array}$ & \multirow{2}{*}{-} & \multirow{2}{*}{ - } & \multirow{2}{*}{-} & $\begin{array}{l}\text { Actinomma } \\
\text { plasticum }\end{array}$ & & \\
\hline & $184.8-187.0 \mathrm{mbsf}$ & $182.3-186.0 \mathrm{mbsf}$ & & & & Interval zone & \multirow{2}{*}{$\begin{array}{l}\text { Last consistent } \\
\text { occurrence of } \\
\text { C. ampullacea }\end{array}$} & \multirow{2}{*}{$\sim 14.7$} \\
\hline & $\begin{array}{l}22-2,75-77 \mathrm{~cm} \\
\text { to } 22-3,75-77 \mathrm{~cm}\end{array}$ & $\begin{array}{c}23-3,24-26 \mathrm{~cm} \\
\text { to } 23-\mathrm{cc}\end{array}$ & $2-1,25-27 \mathrm{~cm}$ & - & - & $\begin{array}{l}\text { C. Kladaros Subzone b. } \\
\text { (Cyrtocapsella ampullacea) }\end{array}$ & & \\
\hline & $188.5-190.0 \mathrm{mbsf}$ & $187.5-192.4 \mathrm{mbsf}$ & $190.2 \mathrm{mbst}$ & 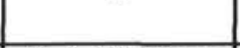 & & Taxon range subzone & \multirow{2}{*}{$\begin{array}{l}\text { First occurrence of } \\
\text { C. ampullacea }\end{array}$} & \multirow{2}{*}{$\sim 14.8$} \\
\hline & $\begin{array}{c}22-4,75-77 \mathrm{~cm} \\
\text { to } 22-5,75-77 \mathrm{~cm}\end{array}$ & $\begin{array}{l}24-1,25-27 \mathrm{~cm} \\
\text { to } 24-2,25-27 \mathrm{~cm}\end{array}$ & $\begin{array}{c}2-2,25-27 \mathrm{~cm} \\
\text { to } 2-4,25-27 \mathrm{~cm}\end{array}$ & $\begin{array}{c}13-4,104-106 \mathrm{~cm} \\
\text { to } 13-\mathrm{cc}\end{array}$ & \multirow{2}{*}{-} & $\begin{array}{l}\text { C. kladaros Subzone a. } \\
\text { (Cyrtocapsella kladaros) }\end{array}$ & & \\
\hline & $191.5-193.0 \mathrm{mbsf}$ & $192.7-194.2 \mathrm{mbsf}$ & $191.7-194.7 \mathrm{mbsf}$ & 115.4-119.3mbsf & & Partial range subzone & \multirow{2}{*}{$\begin{array}{c}\begin{array}{c}\text { Cyrtocapsella } \\
\text { kladaros }\end{array} \\
\end{array}$} & \multirow{2}{*}{$\sim 14.9$} \\
\hline & $\begin{array}{l}22-6,75-77 \mathrm{~cm} \\
\text { to } 22-\mathrm{cc}\end{array}$ & $\begin{array}{l}24-3,25-27 \mathrm{~cm} \\
\text { to } 24-4,25-27 \mathrm{~cm}\end{array}$ & $2-6,25-27 \mathrm{~cm}$ & $\begin{array}{l}14-1,109-111 \mathrm{~cm} \\
\text { to } 15-4,25-27 \mathrm{~cm}\end{array}$ & \multirow{2}{*}{-} & Ceratocyrtis broeggeri & & \\
\hline & $194.5-196.0 \mathrm{mbsf}$ & $195.7-197.2 \mathrm{mbsf}$ & $197.7 \mathrm{mbsf}$ & $120.4-133.6 \mathrm{mbsf}$ & & Interval zone & \multirow{2}{*}{$\begin{array}{c}\text { irst occurrence of } \\
\text { Ceratocyrtis } \\
\text { stoermeri }\end{array}$} & \multirow{2}{*}{$\sim 15.5$} \\
\hline & $\begin{array}{l}23-1,75-77 \mathrm{~cm} \\
\text { to } 23-3,75-77 \mathrm{~cm}\end{array}$ & $\begin{array}{c}24-5,25-27 \mathrm{~cm} \\
\text { to } 24-\mathrm{cc}\end{array}$ & $\begin{array}{c}2-\mathrm{cc} \\
\text { to } 3-2,25-27 \mathrm{~cm}\end{array}$ & \multirow{2}{*}{ - } & \multirow{2}{*}{-} & Cyrtocapsella eldholmi & & \\
\hline & $196.8-199.8 \mathrm{mbsf}$ & $198.7-199.6 \mathrm{mbsf}$ & $199.7-201.4 \mathrm{mbsf}$ & & & Partial range zone & \multirow{2}{*}{$\begin{array}{c}\text { First occurrence of } \\
\text { Cyrtocapsella } \\
\text { eldholmi }\end{array}$} & \multirow{2}{*}{$\sim 15.7$} \\
\hline & - & - & - & $\begin{array}{c}15-5,105-107 \mathrm{~cm} \\
\text { to } 17-\mathrm{cc}\end{array}$ & \multirow{2}{*}{-} & Ceratocyrtis manumi & & \\
\hline & & & & 135.9-148.5mbsf & & & \multirow{4}{*}{$\begin{array}{l}\text { First occurrence of } \\
\text { feratocyrtis manumi } \\
\begin{array}{l}\text { First occurrence of } \\
\text { C. davisiana } \\
\text { cornutoides }\end{array} \\
\end{array}$} & \multirow{2}{*}{$\begin{array}{c}\sim 16.0- \\
16.2 \\
\end{array}$} \\
\hline \multirow{18}{*}{$\underset{\$}{\Phi}$} & & & & \begin{tabular}{|c|}
$18-1,105-107 \mathrm{~cm}$ \\
to $20-2,105-107 \mathrm{~cm}$
\end{tabular} & \multirow[b]{2}{*}{-} & Cycladophora davisiana & & \\
\hline & - & - & - & $\begin{array}{r}\text { to } 20-2,105-10 / \mathrm{cm} \\
158.4-178.9 \mathrm{mbsf}\end{array}$ & & Partial range zone & & \multirow{2}{*}{$\sim 16.7$} \\
\hline & - & & & \begin{tabular}{|c|}
$20-3,105-107 \mathrm{~cm}$ \\
to $20-5,105-107 \mathrm{~cm}$
\end{tabular} & & Spongotrochus vitabilis & & \\
\hline & - & - & - & $180.4-183.4 \mathrm{mbsf}$ & - & Partial range zone & & $\sim 168$ \\
\hline & - & & & $\begin{array}{c}20-6,105-107 \mathrm{~cm} \\
\text { to } 20-\mathrm{cc}\end{array}$ & - & Interzone A & $\begin{array}{l}\text { pongourocnus } \\
\text { vitabilis }\end{array}$ & \\
\hline & 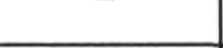 & - & - & $184.9-185.8 \mathrm{mbsf}$ & - & & $\begin{array}{l}\text { Last consistent } \\
\text { occurrence of }\end{array}$ & $\sim 16.9$ \\
\hline & $\begin{array}{c}23-4,75-77 \mathrm{~cm} \\
\text { to } 23-\mathrm{cc}\end{array}$ & - & $\begin{array}{c}3-3,25-27 \mathrm{~cm} \text { to } \\
3-\mathrm{cc}\end{array}$ & $\begin{array}{l}21-1,35-37 \mathrm{~cm} \text { to } \\
23-4,105-107 \mathrm{~cm}\end{array}$ & _- & $\begin{array}{l}\text { Pseudodictyophimus } \\
\text { amundseni }\end{array}$ & P. amundseni & \\
\hline & 201.3-205.6mbsf & & $202.9-207.5 \mathrm{mbsf}$ & $186.2-210.7 \mathrm{mbsf}$ & & Taxon range zone & First occurrence of & $\sim 175$ \\
\hline & $\begin{array}{l}24-1,75-77 \mathrm{~cm} \\
\text { to } 24-5,75-77 \mathrm{~cm}\end{array}$ & - & $\begin{array}{c}4-1,24-26 \mathrm{~cm} \\
\text { to } 4-6,24-26 \mathrm{~cm}\end{array}$ & $\begin{array}{c}23-5,105-107 \mathrm{~cm} \text { to } \\
25-3,105-107 \mathrm{~cm}\end{array}$ & - & $\begin{array}{l}\text { Corythospyris jubata } \\
\text { jubata severdrupi }\end{array}$ & P. amundseni & 11.0 \\
\hline & 206.4-212.4mbsf & & 209.4-217.0mbsf & 212.2-228.8mbsf & & Partial range zone & First occurrence of & $\sim 17.8$ \\
\hline & & - & & $\begin{array}{c}25-4,105-107 \mathrm{~cm} \\
\text { to } 27-\mathrm{cc}\end{array}$ & - & $\begin{array}{l}\text { Clathrospyris sandellae } \\
\text { Partial range zone }\end{array}$ & & \\
\hline & $213.0-221.1 \mathrm{mbsf}$ & & 218.9-228.5mbsf & 230.3-254.0mbst & & & $\begin{array}{l}\text { toccurrence of } \\
\text { lathrospyris }\end{array}$ & $\sim 18.5$ \\
\hline & - & - & $\begin{array}{c}6-1,30-32 \mathrm{~cm} \\
\text { to } 8-3,25-27 \mathrm{~cm}\end{array}$ & $\begin{array}{c}28-1,105-107 \mathrm{~cm} \\
\text { to } 30-\mathrm{cc}\end{array}$ & - & $\begin{array}{l}\text { Gondwanaria japonica } \\
\text { kiaeri }\end{array}$ & sandellae & 10.0 \\
\hline & & - & 228.8-251.5mbsf & 255.2-274.2mbsf & - & Partial range zone & First occurrence of & $\sim 20.0-$ \\
\hline & - & - & $\begin{array}{c}8-4,25-27 \mathrm{~cm} \\
\text { to } 9-\mathrm{cc}\end{array}$ & - & - & Eucyrtidium saccoi & & 20.5 \\
\hline & 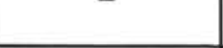 & 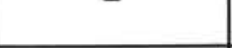 & $253.0-267.1 \mathrm{mbsf}$ & - & - & Partial range zone & nce of & $\sim 21.2$ \\
\hline & - & - & $\begin{array}{l}10-1,25-27 \mathrm{~cm} \\
\text { to } 11-1,65-67 \mathrm{~cm}\end{array}$ & - & - & $\begin{array}{c}\text { Actinomma henningsmoen } \\
\text { Partial range zone }\end{array}$ & & 21.2 \\
\hline & & & 267.4-277.5mbsf & & & & $\begin{array}{l}\text { rst occurrence of } \\
\text { henningsmoeni }\end{array}$ & $\sim 21.7$ \\
\hline
\end{tabular}


Similarly, Pseudodictyophimus has a long local record (extending to the Holocene), but transition stages leading to $P$. amundseni morphology have not been observed. The early history of this species is presently unknown, and it is never abundant in our samples, which suggests that $P$ amundseni may have been an expatriate. Interestingly, the extinction level of $P$ amundseni appears to differ at Sites 642 and 643. In Hole 643A this species disappears below the base of the Spongotrochus vitabilis Zone (Table 5, Col. 14), but $P$. amundseni ranges up to the base of the Ceratocyrtis eldholmi Zone at Site 642 (Table 2, Col. 14). The latter occurrences may represent displaced specimens, however. We infer a hiatus at the base of the $C$. eldholmi Zone at Site 642 , and the specimens of $P$. horrida in the base of this zone may be redeposited from the underlying $P$. horrida Zone sediments. We infer that sediments in the sequence ranging from the $A$. henningsmoeni Zone to the $P$. amundseni Zone were deposited during an interval when the water column overlying the Vøring Plateau had a relatively warm-water surface layer, and significant proportions of the radiolarian faunas represent northern high- latitude morphs of warm-cosmopolite populations or local evolutionary products derived from stocks transported from the south.

A contrary explanation is offered here for the immediately overlying sequence in Hole $643 \mathrm{~A}$, which includes an interzone and three radiolarian zones not observed at Site 642 . Interzone A represents a short interval of exceptionally low diversity in the radiolarian fauna. Spongotrochus vitabilis is a short-ranging species, and its origin is presently unknown. Cycladophora davisiana cornutoides appears abruptly in high but variable frequencies in Section 104-643A-20X-2 and has a discontinuous occurrence in declining frequencies to the top of the Ceratocyrtis broeggeri Zone (Section 104-643A-14H-1) (Table 5, Col. 17). Under various nomenclature, this species has been recorded in Neogene sediments of the North Pacific and Antarctic. At DSDP Site 173 in the eastern North Pacific, Kling (1973) recorded C. davisiana cornutoides (referred to as Theocalyptra $\mathrm{sp}$.) as having an essentially continuous range from the Holocene to lower Miocene (planktonic foraminifera zone N5/N6). The base of the range of this species appears to be highly diachronous in the western North Pacific. Ling (1973) recorded the first occurrences of Theocalyptra sp. as varying from upper Miocene at DSDP Site 192 to lower Miocene in DSDP Site 186. We regard these inconsistent basal ages as resulting from variations in moderately cold-water mass distributions. Possibly, C. davisiana cornutoides invaded the Norwegian Sea only during an interval when North Atlantic water was excluded from this region. Its first occurrence in Hole $643 \mathrm{~A}$ is placed within upper N6. In the Antarctic, Petrushevskaya (1975) reported this species (referred to as Diplocyclus sp. bicorona) as ranging down to lowest Miocene sediments at DSDP Site 278.

Ceratocyrtis manumi and Ceratocyrtis broeggeri also have sudden first appearances in Sections 104-643A-17X-CC and 104$643 \mathrm{~A}-14 \mathrm{H}-4$, respectively (Table 5, Cols. 19 and 22 ), and we infer preglacial-Arctic origins for these species because of their association with $C$. davisiana cornutoides. The absolute base of the $C$. broeggeri Zone was not observed in Hole 643A, because of the absence of Ceratocyrtis stoermeri in our samples. Perhaps this short-ranging species occurs in the unsampled intervals of Hole 643A. If $C$. davisiana cornutoides is indicative of cool surface-water temperatures, as we infer, then the $C$. davisiana cornutoides to $C$. broeggeri Zones of Hole 643A (and also possibly the $S$. vitabilis and Interzone A) represent an interval spanning the early/middle Miocene boundary when the water column overlying the Vøring Plateau may have been influenced predominantly by polar currents. The volume of North Atlantic water entering the Norwegian Sea appears to have been very much reduced. We suggest that these conditions may have been the result of the three sea-level low stands associated with the bases of Cycles TB2.2, TB2.3, and TB2.4 of Haq et al. (1987) (Goll, this volume, Fig. 6). Sea-level lowerings may have triggered submarine erosion on the Vøring Plateau, which may explain the absence of this presumably polar-dominated sedimentary sequence at Site 642. Full arctic conditions may not have persisted throughout this interval, however. C. davisiana cornutoides is sparse or absent in the uppermost $C$. manumi Zone and the lower $C$. broeggeri Zone in Core 104-643A-15H, and it was during that interval (represented by a short hiatus at Hole $643 \mathrm{~A}$ ) that the Cyrtocapsella eldholmi Zone and basal $C$. broeggeri Zone were deposited at Site 642. Trace occurrences of $C$. davisiana cornutoides and $C$. manumi were observed in the $C$. broeggeri Zone of Site 642 .

The sequence including the Cyrtocapsella kladaros Zone, Actinomma plasticum Zone, Pseudodictyophimus horrida Zone, and Interzone B is probably complete at Site 642 . We infer a hiatus of unknown duration between the top of the $C$. broeggeri Zone of Site 643 and the base of the $C$. kladaros Zone of Site 642. Only portions of this sequence are present at Site 643 . The C. ampullacea Subzone and the Actinomma plasticum Zone are completely absent. The rare and sporadic occurrences of Clathrospyris sandellae associated with abundant but variable occurrences of Hexalonche nakasekoi suggest that the $P$. horrida Zone of Site 643 is coeval with the lower $P$. horrida Zone of Site 642 .

The $C$. kladaros Zone represents an interval of rapid faunal turnover for the radiolarians, which may have resulted from renewed influx of North Atlantic water followed by evolutionary readjustment of temperate lineages to Norwegian Sea environments. Cyrtocapsella kladaros and Cyrtocapsella amipullacea appear to replace each other in succession (Table 2, Cols. 23 and 24; Table 3, Cols. 17 and 18). A. plasticum is an easily recognized species and is an excellent recognition criterion for the $A$. plasticum and $P$. horrida Zones. The species has discontinuous occurrences at the top and bottom of its range and consequently can not be used as a boundary marker. The top of the $P$. horrida Zone in Holes $642 \mathrm{~B}$ and $642 \mathrm{C}$ is marked by the last consistent occurrence of $P$. horrida. We have found no species to characterize the overlying Interzone $\mathrm{B}$, although $P$. horrida has sporadic occurrences in this interval, and traces of this species have been detected as high as the Tessarastrum thiedei Zone.

The appearance of evolutionarily advanced specimens of Eucoronis fridtjofnanseni at Sites 642 and 643 is so profound that we are convinced of the presence of a hiatus at the base of its range. The timing of this presumed erosive event, which is associated with the onset of persistent calcite preservation, cannot be deduced from radiolaria biostratigraphy, but it may be related to the offlap at the base of cycle TB2.5 of the sequence stratigraphy of Haq et al. (1987). The age of the short interval from Section 104-642B-19H-2 to -19H-5 and from Section 104$642 \mathrm{C}-19 \mathrm{H}-6$ to $-20 \mathrm{H}-2$ is very imperfectly understood, but the presence of calcareous microfossils and improved polar reversal records in the overlying sequence at Site 642 and coeval sediments at Site 643 allow for more confident age assignment. $E$. fridtjofnanseni is obviously closely related to $C$. sandellae, but the intermediate morph, Clathrospyris vogt $i$ is restricted to rare occurrences in the C. vogti Zone of Site 643 (Table 5, Col. 29). The extinction level of $C$. sandellae is coincident with or slightly older than the extinction level of $E$. fridtjofnanseni. Corythospyris reuschi adopts a morphology that appears to mimic $E$. fridtjofnanseni, and $C$. reuschi is very abundant for a short interval following the last consistent occurrence of $E$. fridtjofnanseni (the $C$. reuschi Zone; Table 2, Col. 29; Table 5, Col. 31).

The stratigraphic relationship of the recovery in the overlying interval ranging from the Spongurus cauleti Zone to the Antarctissa whitei Zone is similar to the interval ranging from Inter- 
zone A to the $P$. horrida Zone. A major hiatus at Site 642 is represented by an almost complete sedimentation record at Site 643 , and a younger hiatus in Hole $643 \mathrm{~A}$ is represented by possibly almost complete sedimentation at Site 642 . Recovery at the two sites do not completely overlap, however, and the timing of these events bears no obvious relationship to the sequence stratigraphy of Haq et al. (1987). The $S$. cauleti and $H$. esmarki Zones are well represented at Site 643. However, Cyrtocapsella japonica, which is dominant in one sample from the base of the S. cauleti Zone in Hole 642B (Table 2, Col. 33), and has low frequencies in the upper $C$. reuschi Zone to lower $S$. cauleti Zone of Hole $642 \mathrm{C}$ (Table 3, Col. 27), is not observed in coeval sediments of Hole 643A. The $S$. cauleti Zone and $H$. esmarki Zone of Site 642 are very short and possibly condensed. The relationship of the H. esmarki Zone of Site 642 to that of Site 643 is unclear. At Site $643, S$. cauleti and $H$. esmarki overlap broadly and the lower $H$. esmarki Zone is characterized by abundant $H$. esmarki and Sipocampe arachnea. In Hole 642B, S. cauleti and $H$. esmarki do not overlap, and $S$. arachnea is absent.

The hiatus in Core 104-642B-15H is directly overlain by the Tessarastrum thiedei Zone. In Hole $642 \mathrm{C}$, the T. thiedei Zone is preceeded by the very short Larcospira bulbosa Zone. The stratigraphic relations of the $L$. bulbosa and $T$. thiedei Zones are unclear. An undescribed and unreported species, probably ancestral to $T$. thiedei, occurs in the $L$. bulbosa Zone, but the first occurrence of T. thiedei in Section 104-642C-15H-6 (Table 3, Col. 34 ) is quite abrupt, and there are no evolutionary intermediates. Presumably, the $L$. bulbosa Zone sediments of Hole $642 \mathrm{C}$ are separated from the overlying interval by a short hiatus. The evolutionary history of $T$. thiedei is unknown, although the species has a long-ranging occurrence in high frequencies. Phormospyris thespios has its first appearance in the L. bulbosa Zone of Hole $642 \mathrm{C}$ (Table 2, Col. 31) and is present in very low frequencies in the base of the T. thiedei Zone of Holes 642B (Table 2, Col. 36) and 642C.

Within the T. thiedei Zone and Liriospyris cricus Zone, the radiolaria fauna begins to acquire a more modern aspect. This assemblage includes Lophospyris pentagona quadriforis and Antarctissa whitei, which range up to the top of the Spongaster ?tetras Zone. However, extant species such as Hexacontium pachydermum, Phorticium clevei, and Ceratocyrtis histricosus also have their first occurrences in this interval. Phaeodaria such as Euphysetta elegans and Challengeron diodon appear to have inconsistent ranges in Holes $642 \mathrm{~B}$ and $642 \mathrm{C}$, but occurrences of these species are largely a function of preservation conditions. The first occurrence of Pseudodictyophimus gracilipes tetracanthus is the youngest unquestionable biostratigraphic event for the radiolarians that we have recorded in Holes 642B (Table 2, Col. 47) and $642 \mathrm{C}$ (Table 3, Col. 42). The youngest recorded occurrence of this species is in Section 104-644A-19H-CC (Table 6, Col. 4).

In Leg 104 recovery, the Spongaster ?tetras Zone and Cycladophora davisiana davisiana Zone occur only at Site 644. The taxonory of $S$. ?tetras is provisional. The species is present in the oldest recovery of Site 644 (Table 6, Col. 3), and its ancestry is unclear. The morphology of this species is closely similar to $S$. tetras, but it differs from the latter in that the skeleton is not simply composed of spongy lattice with bulbous extensions at the apices. In contrast, the morph in Site 644 sediments has four distinct radial arms of unequal length disposed in an irregular cruciform configuration and loosely interjoined by a patagial fringe (Pl. 4, Figs. 35-36). The stratigraphic range of $S$. ?tetras does not extend above the preglacial sediments of Hole $644 \mathrm{~A}$. A glacial bed approximately $4.5 \mathrm{~m}$ thick, which is barren of radiolarians, separates the S.?tetras Zone and $C$. davisiana davisiana Zone. This contact coincides with the base of the glacial marine section in Hole 644A (Unit I) and has an estimated age of $2.56 \mathrm{Ma}$. In the North Pacific, C. davisiana davisiana first occurs at 3.3 Ma in the Lamprocyclas heteroporus Zone of DSDP Hole 580 (Morley, 1985). Its younger and highly sporadic occurrence in Hole 644A, without an obvious antecedent, is a further indication that $C$. davisiana davisiana must be restricted in its distribution to cold-water environments.

The distribution of Eucyrtidium biconicum appears to indicate zoogeographic differentiation between Sites 642 and 643 . $E$. biconicum is closely related to the $E$. saccoi species complex, but differs in its specialized chamber configuration and longitudinal ridge development. The species has approximately isochronous advent levels at Sites 642 and 643, but it ranges higher at Site 642 than at Site 643. In Hole 642D, E. biconicum first occurs at the top of the C. sandellae Zone (Table 4, Col. 13) in trace to sparse frequencies (abundant in Section 104-642D-3X$1)$, and the species ranges sporadically in Hole 642B up to the A. plasticum Zone (Table 2, Col. 4, not recorded in Hole 642C). $E$. biconicum has a more consistent occurrence in Hole 643A to the top of the $C$. manumi Zone (Table 5, Col. 13), but the species occurs above this horizon discontinuously only in a short interval of the $C$. broeggeri Zone.

Occurrences of collosphaerids are of particular interest, because these colonial radiolarians are commonly regarded as indicators of warm surface water conditions. The collosphaerid faunule in Leg 104 sediments is restricted to four species, of which two are undescribed and extremely rare. We have documented the distributions of Trisolenia megalactis and Acrosophaera spinosa echinoides at Sites 642 and 643. T. megalactis has sporadic occurrences in trace to rare frequences in intervals of the $C$. manumi and $C$. broeggeri Zones of Hole 643A (Table 5, Col. 21), for which there are no coeval sediments at Site 642. A. spinosa echinoides has isolated trace occurrences in the $C$. sandellae, $C$. davisiana cornutoides, and $C$. broeggeri Zones of Hole 642B (Table 2, Col. 11), Hole 642C (Table 3, Col. 14), and Hole 643A (Table 5, Col. 18). The species has common to abundant occurrences in the $P$. horrida Zone in each of these holes. These distributions are not easily interpreted. Whereas we infer temperate conditions for the $P$. horrida Zone, we regard the Norwegian Sea as having been generally cold during the $C$. manumi and $C$. broeggeri Zones. In Hole $643 \mathrm{~A}$, both of these collosphaerids co-occur with a presumptive cold-water indicator species, C. davisiana cornutoides (Table 5, Col. 17).

A few species in the Neogene radiolarian faunas of Leg 104 undergo marked frequency oscillations, which may be attributed to paleoceanographic parameters. We will comment briefly on the distributions of two species: Hexalonche ?nakasekoi and Siphocampe arachnea. $H$. ?nakasekoi is present in trace to sparse frequencies in the oldest biosiliceous recovery of Hole 642D (Table 4, Col. 5), and high-frequency peaks ranging from abundant to very dominant commence in the G. japonica kiaeri Zone. The species is most consistently numerous in the $C$. jubata sverdrupi to $P$. amundseni Zones (Table 5, Col. 4), and it experienced additional prolonged pulses during the $C$. broeggeri to $C$. kladaros Zones and during the P. horrida Zone (Table 5, Col. 4, and Table 2, Col. 5). H. ?nakasekoi was also reported as dominant within a four meter interval at the base of the $E$. fridtjofnanseni Zone of Hole 341 by Goll and Bjørklund (1980, referred to as Hexalonche sp. A). These major long-term peaks appear to coincide with intervals of relatively high sea-level sands (see Goll, this volume). H. ?nakasekoi is abundant in its last occurrence in Hole 642B (E. fridtjofnanseni/C. reuschi Zone; Table 2, Col. 5), but the species has sporadic low-frequency occurrences as high as the $S$. cauleti Zone in Hole 643A (Table 5, Col. 4) and Hole 642C (Table 4, Col. 5).

Siphocampe arachnea has sporadic occurrences in trace to sparse frequences in sediments as old as the $A$. henningsmoeni Zone (Table 4, Col. 7), and it has its oldest high-frequency peak in 
our samples near the top of the G. japonica kiaeri Zone of Hole 643A (Table 5, Col. 8). High-frequency peaks reappear in the $P$. amundseni Zone, and there are 16 such peaks (generally singlesample anomalies) between this zone and the $A$. whitei Zone in the composite recoveries of Sites 642 and 643 (Table 2, Col. 8; Table 3, Col. 6; and Table 5, Col. 8). These peaks are particularly pronounced and extended during the $C$. broeggeri to $C$. kladaros Zones and $S$. cauleti to $H$. esmarki Zones.

The general subject of pronounced frequency oscillations in Neogene faunal components requires further study. These and other species must be examined in expanded sample sets to compare radiolarian distributions with data on other microfossils (i.e., Ciesielski and Case, this volume; Locker and Martini, this volume), both within and beyond the Norwegian Sea with the objective of reconstructing high northern latitude paleoenvironments.

\section{CORRELATIONS TO DSDP LEG 38 SEDIMENTS}

To the extent to which they re presently known, the time stratigraphic distributions of Leg 38 sediments are shown in Figure 2. Only the Neogene biosiliceous intervals of Sites 338, 341,342 , and 348 are presented here, and these sediments can be correlated to the radiolarian biozonation established on the basis of Leg 104 material. The ages of Leg 104 cores follow the convention of the biostratigraphic synthesis, although there is possibly a slight discrepancy in these time correlations as will be discussed here.

The highly discontinuous nature of these Leg 38 recoveries is readily apparent. The most extreme case is Site 342 , which contains only small segments of the Tessarastrum thiedi. Ceratocyrtis manumi, Cycladophora davisiana cornutoides, and probably basal Eucyrtidium saccoi Zones. There are no sediments coeval to Cores $338-4$ and -5 or Holes $642 \mathrm{~B}$ and $642 \mathrm{C}$. The recovery in Hole 338 appears to be only $8 \%$ complete over a time range of 15.9 m.y. (5.5-21.4 Ma). Seven isotope ages for the basalts underlying DSDP Core 341-6 range from 41.3 to $79.9 \mathrm{Ma}$ (Kharin et al., 1976). If the young end of this age range is correct, then basement at Site 342 is approximately coeval with the pyroclastic unit at Site 642, and hiatuses of equal duration occur below the oldest pelagic sediments at each site. The thin unconformity-bounded sediment packages at Site 342 must represent a probably incomplete record of the oldest sediments above consecutive erosional episodes.

The Neogene biosiliceous section at Site 338 includes short segments of Interzone B, and the Pseudodictyophimus horrida, Actinomma plasticum, Cyrtocapsella kladaros, Ceratocyrtis broeggeri, Cyrtocapsella eldholmi, and Pseudodictyophimus amundseni Zones between Cores 338-8 and -12. Core 338-13 appears to contain the evolutionary origination of Gondwanaria japonica kiaeri. The interval from lower Core 338-13 to upper Core 33817 partially fills the hiatus in Section 104-642D-8X-3, although the age of this interval is as yet uncertain because of a possible repeated section. The interval from lower Core 338-17 to upper Core 338-19 is probably assignable to the Actinomma henningsmoeni Zone. Lower Core 338-19 has been correlated to calcareous nannofossil zone NP24 (upper Oligocene) by Müller (1976). As shown in Figure 2, the interval from Core 338-8 to upper Core $338-19$ is approximately $38 \%$ complete over a time range of $8.1 \mathrm{~m} . \mathrm{y}$. (14.0-22.1 Ma).

We infer that the biosiliceous section at Site 341 includes sediments having ages not represented in Leg 104 recovery. Lower Core 341-25 and Core 341-26 belong to the Corythospyris reuschi and Eucoronis fridtjofnanseni/C. reuschi Zones, respectively. The Rb-Sr date of $11.6 \pm 0.2 \mathrm{Ma}$ for Section $341-25-3$ by Smalley et a1.(1986) is not consistent with our results, nor do we support the contention of these authors that Core 341-32 has an approximate age of $18 \mathrm{Ma}$ based on comparison with the global
${ }^{87} \mathrm{Sr} /{ }^{86} \mathrm{Sr}$ curve. Cores $341-27$ to -31 are correlative with the $E$. fridtjofnanseni Zone of the present system (Goll and Bjørklund, 1980). The interval including Cores $341-31$ and -34 is younger than Interzone $\mathrm{B}$, but older than the first appearance of $E$. fridtjofnanseni. A new zone is required to characterize these sediments, which are coeval with the upper Lithomelissa stigi Zone of Bjørklund (1976). The correlation by diatoms (Schrader and Fenner, 1976) of Cores $341-26$ to -34 , with Cores $338-8$ to upper -11 , is in conflict with our radiolarian data. As at Site $642, E$. fridtjofnanseni has a very abrupt first appearance with high frequencies in Core 341-31, and it is reasonable to assume the presence of a hiatus at the base of this core. Carbonates are not persistently present in this interval until Core 341-29.

The upper part of the biosiliceous section at Site 348 (Cores 348-6 to -12) is correlative with the Pseudodictyophimus gracilipes tetracanthus and Antarctissa whitei Zones. Below a major hiatus, there is a $66.5-\mathrm{m}$ non-calcareous interval with highly erratic recoveries (Cores 348-13 to -16) bearing E. fridtjofnanseni. Because of the similar present water depths at Sites 348 and 341 (1763 and $1439 \mathrm{~m}$, respectively), it is assumed that the $E$. fridtjofnanseni Zone sediments at Site 348 must predate Core 34129 , despite the disparate geographic positions of the two localities.

Thus, although confirming evidence is lacking at this time, a case can be made for a younger onset date for persistent Neogene carbonate deposition in the Norwegian Sea than has been adopted in the biostratigraphic synthesis (Goll, this volume. In Leg 104 recovery, the advent levels of $E$. fridtjofnanseni and persistent carbonate deposition are coincident and have been assigned an age of $13.6 \mathrm{Ma}$. By constructing an interpretative composite section from Holes 341 and 348 however, it appears that these two events are separated by at least $66.5 \mathrm{~m}$ of sediment. Moreover, the base of the $E$. fridtjofnanseni Zone must be younger than this assigned age by at least the time required to deposit $29.5 \mathrm{~m}$ of sediment in Hole 341 (Cores 341-32 to -34). On the basis of these considerations, we conclude that more reasonable ages for the base of the $E$. fridtjofnanseni Zone and the onset of persistent carbonate deposition are approximately $12.6 \mathrm{Ma}$ and $10.5 \mathrm{Ma}$, respectively. Consequently, we argue that the thin sediment layer at the base of the $E$. fridtjofnanseni Zone in Cores 104-642B-19H and $-642 \mathrm{C}-19 \mathrm{H} /-20 \mathrm{H}$ has an age of less than $10.5 \mathrm{Ma}$ and should be moved up above the hiatus to a position probably contiguous with the overlying sediments.

The position of the Clathrospyris vogti Zone in this revised scheme is unclear. Although $C$. vogti (referred to as intermediates or evolutionary early stages of $E$. fridtjofnanseni by Goll and Bjørklund, 1980) ranges in low frequencies in Hole 341 between Cores $341-28$ and -31 , we have found no exclusive occurrences of $C$. vogti in Holes 341 or 348.

If these correlations to Leg 38 sites are correct, then sections in Holes 341 and 348 must partially fill the major middle Miocene hiatus of Leg 104 recoveries, and the associated unconformity is not as extensive as might be inferred from Leg 104 results alone. Clearly, however, both these sites experienced one or more major erosional episodes during the late middle Miocene, but the ages and durations of these hiatuses are not isochronous across the Vøring Plateau.

\section{CONCLUSIONS}

On Leg 104, thick sections of Neogene biosiliceous strata were penetrated that had not been previously cored from the Norwegian Sea. With the exception of the past $0.75 \mathrm{Ma}$, radiolarians are consistently present in sediments younger than approximately $22.0 \mathrm{Ma}$ at one or more of the sites, although these microfossils are rarely the dominant biosiliceous component. As in other high-latitude regions as well as some upwelling coastal 
basins, the concentration of radiolarians is generally masked by the more numerous diatoms and locally numerous silicoflagellates and ebridians. Sediments ranging in age from basal Miocene to early Eocene were also recovered on Leg 104, but radiolarians are either absent in these strata or have an insignificant record. Radiolarian preservation is only moderate and occasionally poor for the upper Miocene to Pleistocene sequence, but nevertheless it has been possible to reconstruct from Leg 104 recovery a composite section of radiolarian-bearing sediment that is approximately $72 \%$ complete for the Neogene. Significant hiatuses in Leg 104 recovery provide challenges for future drilling, and a thorough integration of radiolarian biostratigraphies for both Legs 38 and 104 remains incomplete.

The radiolarian faunas of the Norwegian Sea have not been carefully compared with other northern North Atlantic radiolarians of coeval age, but a high frequency of endemism is suspected. Although temperate radiolarians appear to have existed in the water column overlying the Vøring Plateau for much of the Neogene, many other species in Leg 104 sediments have not been recorded elsewhere, and we conclude that they were members of the Arctic/Subarctic fauna or Norwegian Sea endemics. These latter species are interpreted as dominant during episodes of low sea level. Abrupt changes in the Neogene radiolarian faunas of the Norwegian Sea suggest that the distributions of these organisms probably have been strongly influenced by the current systems of this region, which in turn are a function of the variable rates of water-mass exchange across the south-bounding aseismic ridges. A simple association can be seen between both sea level (as presented by Haq et al., 1987) and radiolarian faunal composition and sedimentation history. Falling sea levels appear to have triggered erosive events and reduced or eliminated the incursion of temperate taxa as northward transport of Atlantic water across the Iceland-Faeroe Ridge declined. Rising sea levels may have restored conditions for sediment accumulation and stimulated rapid turnover in the radiolarian faunas as temperate taxa re-entered the Norwegian Sea. These relationships are best exemplified in the interval from the Spongotrochus vitabilis Zone to the Cyrtocapsella eldholmi Zone. Erosion appears to have completely obliterated the record of fauna events associated with the low sea-level stand near the close of the middle Miocene (at or near the base of the Eucoronis fridtjofnanseni Zone). The succession including the Spongurus cauleti Zone to Tessarastrum thiedei Zone records a third example of these associations, although faunal stability was re-established rapidly in this case, and there is little direct evidence of preceeding cool-water isolation during this interval of generally moderate climatic conditions. Only minor sea-level low stands during this interval are indicated by $\mathrm{Haq}$ et al. (1987), and local tectonism or ridge subsidence activity may have had a more decisive role in this youngest history of faunal turnover and interrupted sedimentation.

General trends in abundance of radiolarians and quality of preservation throughout the Neogene recovery of Leg 104 may be related to sedimentation history as well. Gradually declining concentrations of biosiliceous components in upper Miocene to Pliocene sediments of Sites 642 and 643 were observed on the basis of smear-slide descriptions (Eldholm, Thiede, Taylor, et al., 1987). The diluting effect of other sedimentary constituents (i.e., calcite and nonbiogenic clastics) may account for these reduced frequencies of siliceous microfossils, but alternatively these trends may simply reflect declining surface-water fertility throughout the Atlantic Ocean during this time. Another possibility, which we favor, is that these abundances may have resulted from poorer conditions for opal preservation. Preservation quality of radiolarians undergoes a marked decline near the top of the $E$. fridtjofnanseni Zone (ca. 8.5 Ma) at both Sites 642 and 643 . Krissek (this volume) notes a pronounced increase in the quartz- plagioclase-illite association at Site 642 at 130 mbsf, which closely coincides with the decline of radiolarian preservation. Similar upward trends in Hole 643A of increasing relative concentrations of illite derived from moderately weathered acidic source rocks also have been observed by Froget et al. (this volume), although an abrupt transition was not detected. Johnson (1974) attributed degraded radiolarian preservation in surface sediments of the eastern equatorial Pacific to their association with high concentrations of illite derived from terrestrial weathering on Latin America, and we conclude that similar sedimentary associations may have been responsible for the reduced abundance and preservation quality of radiolarians as well as other siliceous microfossils in the Norwegian Sea in sediments younger than 8-9 Ma.

The radiolarian zonation presented here accords well with the interpretation of the synthesis chapter (Goll, this volume) with one important exception. In part, the synthesis interpretation is based on radiolarian data, but no contribution has been possible in the barren intervals (104-642B-lH to $-8 \mathrm{H}$; 104-642C$1 \mathrm{H}$ to $-9 \mathrm{H}-6$; $104-642 \mathrm{D}-11 \mathrm{X}-2$ to $-14 \mathrm{X}-\mathrm{CC} ; 104-643 \mathrm{~A}-1 \mathrm{H}$ to $-7 \mathrm{H}-$ $\mathrm{CC}$ and $-31 \mathrm{X}-1$ to $-60 \mathrm{X}-\mathrm{CC})$. The radiolarian biostratigraphy generally supports the division of Leg 104 recovery into ten subsynthemic units (NSN1.1-NSN2.4). Subsynthem NSN1.1 consists of the Actinomma henningsmoeni Zone and Eucyrtidium saccoi Zone. Subsynthem NSN1.2 includes the succession from the Gondwanaria japonica kiaeri Zone to the Ceratocyrtis manumi Zone. Subsynthem NSN1.3 includes the succession from the Cyrtocapsella eldholmi Zone to the Cyrtocapsella kladaros Subzone b. Subsynthem NSN 1.4 includes the succession from the Actinomma plasticum Zone to Interzone B. Our major dispute is associated with Subsynthem NSN1.5 (the Clathrospyris vogti Zone) and Subsynthem NSN1.6 (the base of the E. fridtjofnanseni Zone). We find no radiolarian evidence to support the NSN1.5/NSN2.1 division. We conclude that Subsynthem NSN1.5 at Site 642 is more appropriately joined to the base of Subsynthem NSN2.1. Subsynthem NSN1.5 is represented in Hole $643 \mathrm{~A}$ only by slump-disturbed sediments of uncertain age. Subsynthem NSN2.1 includes the succession from the upper $E$. fridtjofnanseni Zone to the Hexalonche esmarki Zone. Subsynthem NSN2.2 includes the Larospira bulbosa Zone and the Tessarastrum thiedei Zone. Subsynthem NSN2.3 includes the $\mathrm{Li}$ riospyris cricus Zone Antarctissa whitei Zone and Pseudodictyophimus gracilipes tetracanthus Zone. Subsynthem NSN2.4 at Site 644 includes the Spongaster ?tetras Zone and C. davisiana davisiana Zone.

\section{ACKNOWLEDGMENTS}

Prof. Hans-Joachim Schrader and Dr. Sigurd Locker critically reviewed the manuscript and offered many helpful comments. The authors gratefully acknowledge the financial support received during various phases of this research from Statoil, Elf Aquitaine Norge a/s, and Mobil Exploration Norway Inc. We are also indebted to the Norwegian Research Council for Science and the Humanities for a grant to KRB. RMG expresses special thanks to the Continental Shelf and Petroleum Research Institute, Trondheim, for encouragement and many technical services.

\section{REFERENCES}

Berggren, W. A., Kent, D. V., Flynn, J. J., and Van Couvering, J. A., 1985. Cenozoic geochronology. Geol. Soc. Am. Bull., 96:1407-1418. Bjørklund, K. R., 1974. The seasonal occurrence and depth zonation of radiolarians in Korsfjorden, Western Norway. Sarsia, 56:13-42. 1976. Radiolaria from the Norwegian Sea, Leg 38 of the Deep Sea Drilling Project. In Talwani, M., Udintsev, G., et al., Init. Repts. DSDP, 38: Washington (U.S. Govt. Printing Office), 11011168.

Bjørklund, K. R., and Goll, R. M., 1979. Internal skeletal structures of Collosphaera and Trisolenia: a case of repetitive evolution in the Collosphaeridae (Radiolaria). J. Paleontol., 53: 1293-1326. 
Borgert, A., 1902. Mitteilungen über die Tripyleen-Ausbeute der Plankton-Expedition, I. Neue Medusettidae, Cricoporidae und Tuscaridae. Zool. Jahrb., 2:563-577.

Bütschli, 0., 1882. Beiträge zur Kenntnis der Radiolarienskelette, insbesondere der Cyrtide. Z. Wiss. Zool., 3:485-540.

Campbell, A. S., and Clark, B. L., 1944. Miocene radiolarian faunas from southern California. Geol. Soc. Am. Spec. Pap., 51:1-76.

Dogiel, V. A., and Reschetnyak, V. V., 1952. Materialy po radiolyari yam severzapadnoi chasti tikhogo okeana. Issled. Dal'nevost. Morei SSSR., 3:5-36.

Dzinoridze, R. N., Jousé, A. P., Koroleva-Golikova, G. S., Kozlova, G. E., Nagaeva, G. S., Petruschevskaya, M. G., and Strelnikova, N. I., 1978. Diatom and Radiolarian Cenozoic Stratigraphy, Norwegian Basin, DSDP Leg 38. In Talwani, M., Udintsev, G., et al., Init. Repts. DSDP, 38 (Suppl. Vol.): Washington (U.S. Govt. Printing Office), 289-427.

Ehrenberg, C. G., 1838. Über die Bildung der Kreidefelsen und des Kreidemergels durch unsichtbare Organismen. $\mathrm{Kg}$. Akad. Wiss. Berlin Abh., 1838, 59-147.

1844a. Über 2 neue Lager von Gebirgsmassen aus Infusorien als Meeres-Absatz in Nord-Amerika und eine Vergleichung derselben mit den organischen Kreide-Gebilden in Europa und Afrika. $\mathrm{Kgl}$. Preuss. Wiss., Berlin, Ber. Verh., 57-97.

1844b. Einige vorläufige Resultate seiner Untersuchungen der Proben von der Südpolarreise des Capitain Ross, so wie von den Herren Schayer und Darwin zugekommenen Materialien über das Verhalten des kleinsten Lebens in dem Ozean und den grössten bisher zugänglichen Tiefen des Weltmeers. Kgl. Preuss. Wiss. Berlin, Ber. Verh., 182-207.

1861. Über den Tiefgrund des stillen Oceans zwischen Californien und den Sandwich-Inseln aus bis 15600' Tiefe nach Lieut. Brooke. Monatsber. Kgl. Preuss. Akad. Wiss. Berlin, Jahrg. 1860, 819-833.

1862. Über die Tiefgrund-Verhältnisse des Oceans am Eingange der Davisstrasse und bei Island. Kgl. Preuss. Akad. Wiss. Berlin, Monatsber., 1861, 275-315.

1873a. Mikrogeologischen Studien als Zusammenfassung seiner Beobartungen des kleinsten Lebens der Meeres Tiefgründe aller Zonen und dessen geologischen Einfluss. Kgl. Preuss. Akad. Wiss. Berlin, Abh. 1872, 131-397.

1873b. Mikrogeologische Studien über das kleinste Leben der Meeres-Tiefgründe aller Zonen und dessen geologischen Einfluss. Kgl. Preuss. Akad. Wiss. Berling, Abh., 1872, 131-399.

1875. Fortsetzung der mikropaleontologischen Studien als Gesamt-übersicht der mikroskopischen Paläontologie gleichartig analysierter Gebirgsarten der Erde, mit specieller Rücksicht auf den Polycystinen-Mergel von Barbados. Kgl. Preuss. Akad. Wiss. Berlin, Abh., 1875, 1-226.

Eldholm, O., Thiede, J., Taylor, E., et al., 1987. Proc. ODP, Init. Repts., 104: College Station, TX (Ocean Drilling Program).

Goll, R. M., 1976. Morphological intergradation between modern populations of Lophospyris and Phormospyris (Trissocyclidae, Radiolaria). Micropaleontology, 22:379-418.

1978. Five Trissocyclid radiolaria from Site 338. In: Talwani, M., Udintsev, G., et al., Init. Repts. DSDP, 38 (Suppl. Vol.): Washington (U.S. Govt. Printing Office), 177-191.

Goll, R. M., and Bjørklund, K. R., 1980. The evolution of Eucoronis fridtjofnanseni, n. sp. and its application to the Neogene biostratigraphy of the Norwegian-Greenland Sea. Micropaleontology, 26: 356-371.

, 1985. Nephrospyris knutheieri sp.n., an extant trissocyclid radiolarian (Polycystinea: Nassellarida) from the Norwegian-Greenland Sea. Sarsia 70:103-118.

Haeckel, E., 1861. Fernere Abbildungen und Diagnosen neuer Gattungen und Arten von lebenden Radiolarien des Mittelmeres. $\mathrm{Kgl}$. Preuss. Akd. Wiss. Berlin, Monatsber., 1860, 835-845. Berlin. 1862. Die Radiolarien (Rhizopoda, Radiolaria). Berlin (Reimer),

1881. Entwurf eines Radiolarien-Systems auf Grund von Studien der Challenger-Radiolarien. Jena Z. Naturwiss., 15:418-472. 1887. Report on the Radiolaria collected by the H.M.S. Challenger during the years 1873-1876. Rep. Sci. Results of the voyage of H.M.S. Challenger during the years 1873-1876. Zool. 18(1-2).

Haq, B., Hardenbol, J. and Vail, P. R., 1987. Chronology of fluctuating sea level since the Triassic. Science, 235:1156-1167.
Hays, J. D., 1970. Stratigraphy and evolutionary trends of Radiolaria in North Pacific deep-sea sediments. Geol. Soc. Am. Mem., 126:185218.

Johnson, T. C., 1974. The dissolution of siliceous microfossils in surface sediments of the eastern tropical Pacific. Deep-Sea Res., 21: 851-864.

Jørgensen, E., 1900. Protophyten und Protozoen im Plankton aus der norwegischen Westküste. Bergens Mus. Aarb. 1899, 2:1-112.

1905. The protist plankton and the diatoms in bottom samples. Bergens Mus. Skr. Ser. 1, 7:49-151.

Kharin, G. N., Udintsev, G. B., et al., 1976. K/Ar age of the basalts of Norwegian-Greenland Sea Glomar Challenger Leg 38 DSDP. In Talwani, M., Udintsev, G., et al., Init. Repts. DSDP, 38: Washington (U. S. Govt. Printing Office), 755-761.

Kling, S. A., 1973. Radiolaria from the eastern north Pacific, Deep Sea Drilling Project, Leg 18. In Kulm, L. D., von Huene, R., et al., Init. Repts. DSDP, 18: Washington (U.S. Govt Printing Office), 617-671.

Lazarus, D., Scherer, R. P., and Prothero, D. R., 1985. Evolution of the radiolarian species-complex Pterocanium: A preliminary survey. $J$. Paleontol., 59:183-220.

Ling, H. Y., 1973. Radiolaria: Leg 19 of the Deep Sea Drilling Project. In: Kennett, J. P., Houtz, R. C., et al., Init. Repts. DSDP, 29: Washington (U.S. Govt. Printing Office), 777-797.

Martin, G. C., 1904. Radiolaria. In Maryland Geological Survey, Miocene: Baltimore (Johns Hopkins Press), 447-459.

Martini, E., 1971. Standard Tertiary and Quaternary calcareous nannoplankton zonation. In Farinacci, A. (Ed.), Plankt. Conf. II, Rome 1970. Rome (Technoscienz) 2: 739-785.

Morley, J. J., 1980. Analysis of the abundance variations of the subspecies of Cycladophora davisiana. Mar. Micropleontol., 5:205-214.

Morley, J. J., 1985. Radiolaria from the northwest Pacific, Deep Sea Drilling Project Leg 86. In Heath, G. R., Burckle, L. H., Init. Repts. $D S D P, 86$ : Washington (U.S. Govt. Printing Office), 399-422.

Müller, C., 1976. Tertiary and Quaternary calcareous nannoplankton in the Norwegian-Greenland Sea, DSDP Leg 38. In Talwani, M., Udintsev, G., et al., Init. Repts. DSDP, 38: Washington (U.S. Government Printing Office), 823-842.

Nakaseko, K., 1963. Neogene Cyrtoidea (Radiolaria) from the Isozaki Formation in Ibaraki Prefecture, Japan. Osaka Univ. Sci. Rep., 12: $165-198$.

Nigrini, C., 1977. Tropical Cenozoic Artostrobidae (Radiolaria). Micropaleontology, 23:241-269.

Nigrini, C., and Lombari, G., 1984. A guide to Miocene radiolaria. Spec. Publ., Cushman Found. Foraminiferal Res., 20.

Nigrini, C., and Moore, T. C., 1979. A guide to modern radiolaria. Spec. Publ., Cushman Found. Foraminiferal Res., 16.

Petrushevskaya, M. G., 1967. Biological reports of the Soviet Antarctic Expedition (1955-1958). Acad. Nauk. SSSR., Zool. Inst., Studies of Marine Fauna IV, XII:2-187.

1971. Radiolarians of the oceans. Explorations of the fauna of the seas $I X, X V I I: 3-417$.

1975. Cenozoic radiolarians of the Antarctic, Leg 29, DSDP. In: Kennett, J. P., Houtz, R. E., et al., Init. Repts. DSDP, 29: Washington (U.S. Government Printing Office), 541-675.

1981. Radioljarii otrajada Nassellaria mirovogo okeana. Opredeliteli po Faune SSSR. Izdav. Zool. Inst. Akad. Nauk. SSSR, 128: $1-406$.

Petrushevskaya, M. G., and Bjørklund, K. R., 1974. Radiolarians in Holocene sediments of the Norwegian-Greenland Sea. Sarsia 57:3346.

Petrushevskaya, M. G., and Kozlova, G. E., 1979. Description of radiolarian genera and species. In Strelkov, A. A., and Petrushevskaya, M. G. (Eds.). The history of the Microplankton of the Norwegian Sea (on the Deep Sea Drilling materials). Explorations of the fauna of the seas XXII, XXXI: Leningrad (Academy NAUK), 86-157.

Popofsky, A., 1913. Die Nassellarien des Warmwassergebietes. Deutsche Südpolar Expedition 1901-1903, 14 (Zool. vol. 6):217-416.

Reynolds, R. A., Sakai, T., and Casey, R. E., 1980. Synthesis of radiolaria results from DSDP Legs 56 and 57 and their relation to other North Pacific sections. In Langseth, M., Okada, H., et al., Init. Repts. DSDP, 56, 57, Pt. 2: Washington (U.S Govt. Printing Office), 771-773.

Sakai, T., 1984. Neogene radiolarian datum planes of the equatorial and northern Pacific. In Tkebe, N., and Tsuchi, R., (Eds.), Pacific Neogene Datum Planes: Tokyo (Univ. Tokyo Press), 35-40. 
Sanfilippo, A., Westberg-Smith, M. J., and Riedel, W. R., 1985. Cenozoic Radiolaria. In H. M. Bolli, J. B. Saunders, and K. Perch-Nielsen (Eds.), Plankton Stratigraphy. Cambridge (Cambridge University Press), 631-712.

Schrader, H.-J., and Fenner, J., 1976. Norwegian Sea Cenozoic diatom biostratigraphy and taxonomy. Part II, diatoms at Leg 38, taxonomic references. In Talwani, M., Udintsev, G., et al., Init. Repts. DSDP, 38: Washington (U.S. Govt. Printing Office), 963-1099.

Smalley, P. C., Nordaa, A., and Raaheim, A., 1986. Geochronology and paleothermometry of Neogene sediments from the Vøring Plateau using Sr, C and O isotopes. Earth Planet. Sci. Lett., 78:368378.

Takahashi, K., and Honjo, S., 1981. Vertical flux of radiolaria: A taxon quantitative sediment trap study from the western tropical Atlantic. Micropaleontology, 27:140-190.

Vinassa, P. E., 1900. Radiolaria Miocenici Italiani. R. Acad. Sci. Bologna Mem., Ser. 5, 8:565-595.

Westberg-Smith, M. J., and Riedel, W. R., 1984. Radiolarians from the western margin of the Rockall Plateau: Deep Sea Drilling Project Leg 81. In Roberts, D. G., Schnitker, D., et al., Init. Repts. DSDP, 81: Washington (U.S. Govt. Printing Office), 479-501.

\section{APPENDIX \\ Taxa List}

The following is an alphabetical list of all 55 species treated in this paper, including the 21 new species. The original citation as well as a more modern reference is given for each of the previously described species. The location of each species in the stratigraphic range charts for Holes $642 \mathrm{~B}, 642 \mathrm{C}, 642 \mathrm{D}, 643 \mathrm{~A}$, and $644 \mathrm{~A}$ is indicated by column number in Table 8 .

Acrosphaera spinosa echinoides (Haeckel)

Collosphaera echinoides Haeckel, 1862, p. 536, Pl. 34, Figs. 12-13

Acrosphaera spinosa echinoides Bjørklund and Goll, 1979, p. 1311-

1312, P1. 1, Figs. 7, 10-13

Actinomma henningsmoeni $\mathrm{n}$. sp.

Actinomma holtedahli Bjørklund, 1976, p. 1121, Pl. 20, Figs. 8-9

Actinomma livae, n. sp.

Actinomma mirabile, n. sp.

Actinomma plasticum, n. sp.

Antarctissa whitei Bjørklund, 1976, p. 1125, Pl. 15, Figs. 12-17

Artostrobus joergenseni Petrushevskaya, 1971, Fig. 92 VIII-IX; Bjørklund, 1976, Pl. 11, Figs. 12-13

Botryostrobus auritus (Ehrenberg)

Lithocampe aurita Ehrenberg, 1844a, p. 84; Ehrenberg, 1844b, Pl. 22, Fig. 25

Table 8. Location by column number in Tables 2-6 for the 55 species included in this study.

\begin{tabular}{|c|c|c|c|c|c|}
\hline & $\begin{array}{c}\text { Hole 642B } \\
\text { (Table 2) }\end{array}$ & $\begin{array}{c}\text { Hole } 642 \mathrm{C} \\
\text { (Table 3) }\end{array}$ & $\begin{array}{c}\text { Hole 642D } \\
\text { (Table 4) }\end{array}$ & $\begin{array}{c}\text { Hole 643A } \\
\text { (Table 5) }\end{array}$ & $\begin{array}{l}\text { Hole 644A } \\
\text { (Table 6) }\end{array}$ \\
\hline Acrosphaera spinosa echinoides & 11 & 14 & 17 & 18 & - \\
\hline Actinomma henningsmoeni & - & - & 1 & - & - \\
\hline Actinomma holtedahli & 12 & 16 & 6 & 15 & - \\
\hline Actinomma livae & 10 & - & 18 & 20 & - \\
\hline Actinomma mirabile & 22 & 15 & 21 & 23 & - \\
\hline Actinomma plasticum & 25 & 20 & - & 25 & - \\
\hline Antarctissa whitei & 44 & 41 & 2 & - & 2 \\
\hline Artostrobus joergenseni & 43 & 26 & 9 & 33 & 9 \\
\hline Botryostrobus auritus & 30 & 23 & - & 34 & 7 \\
\hline Ceratocyrtis broeggeri & 21 & 13 & 20 & 22 & - \\
\hline Ceratocyrtis histricosus & 42 & 39 & 5 & - & 5 \\
\hline Ceratocyrtis manumi & 18 & - & - & 19 & - \\
\hline Ceratocyrtis stoermeri & 19 & 11 & - & - & - \\
\hline Challengeron diodon & 45 & 43 & - & - & - \\
\hline Clathrospyris sandellae & 6 & 4 & 12 & 10 & - \\
\hline Clathrospyris vogti & - & - & - & 29 & - \\
\hline Corythospyris fiscella & 3 & 8 & 4 & 2 & - \\
\hline Corythospyris hispida & 26 & 19 & 19 & 11 & - \\
\hline Corythospyris jubata jubata & 1 & - & 2 & 3 & - \\
\hline Corythospyris jubata sverdrupi & 13 & - & 14 & 12 & - \\
\hline Corythospyris reuschi & 29 & 24 & - & 31 & - \\
\hline Cycladophora davisiana cornutoides & 17 & 12 & 17 & 17 & - \\
\hline Cycladophora davisiana davisiana & - & - & - & - & 10 \\
\hline Cyrtocapsella ampullacea & 24 & 18 & 23 & - & - \\
\hline Cyrtocapsella eldholmi & 15 & 2 & 16 & 24 & - \\
\hline Cyrtocapsella kladaros & 23 & 17 & 22 & 26 & - \\
\hline Cyrtocapsella japonica & 33 & 27 & - & - & - \\
\hline Cyrtocapsella tetrapera & 9 & 10 & 8 & 7 & - \\
\hline Eucoronis fridtjofnanseni & 28 & 22 & - & 30 & - \\
\hline Eucyrtidium biconicum & 4 & - & 13 & 13 & - \\
\hline Eucyrtidium calvertense & 46 & 37 & - & - & - \\
\hline Eucyrtidium saccoi & 7 & 3 & 10 & 5 & - \\
\hline Euphysetta elegans & 48 & 36 & - & - & - \\
\hline Gondwanaria japonica kiaeri & - & - & 11 & 1 & - \\
\hline Hexacontium pachydermum & 38 & 35 & - & - & 8 \\
\hline Hexalonche esmarki & 35 & 29 & - & 35 & - \\
\hline Hexalonche ?nakasekoi & 5 & 5 & 5 & 4 & - \\
\hline Larcospira bulbosa & - & 30 & - & 37 & - \\
\hline Liriospyris cricus & 41 & 40 & - & - & - \\
\hline Lophospyris pentagona quadriforis & 39 & 33 & - & 38 & 1 \\
\hline Phormospyris thespios & 36 & 31 & - & 39 & - \\
\hline Phorticium clevei & 40 & 38 & - & 41 & - \\
\hline Pseudodictyophimus amundseni & 14 & 1 & 15 & 14 & - \\
\hline Pseudodictyophimus gracilipes tetracanthus & 47 & 42 & - & - & 4 \\
\hline Pseudodictyophimus horrida & 27 & 21 & - & 27 & - \\
\hline Pterocanium korotnevi & 31 & 25 & - & 28 & - \\
\hline Siphocampe arachnea & 8 & 6 & 7 & 8 & 6 \\
\hline Spongaster ?tetras & - & - & - & - & 3 \\
\hline Spongocore puella & 20 & 7 & 9 & 9 & - \\
\hline Spongotrochus vitabilis & 16 & 9 & - & 16 & - \\
\hline Spongurus cauleti & 34 & 28 & - & 32 & - \\
\hline Stichocorys ?delmontensis & 32 & 32 & - & 36 & - \\
\hline Tessarastrum thiedei & 37 & 34 & - & 40 & - \\
\hline Trisolenia megalactis megalactis & - & - & - & 21 & - \\
\hline Velicuccullus oddgurneri & 2 & - & 3 & 6 & - \\
\hline
\end{tabular}


Botryostrobus auritus-australis Group, Nigrini, 1977, p. 246-248, Pl. 1, Figs. 2-5

Ceratocyrtis broeggeri, n. sp.

Ceratocyrtis histricosus (Jørgensen)

Helotholus histricosus Jørgensen, 1905, p. 135, Pl. 16, Figs. 86-88

Ceratocyrtis histricosus Bjørklund 1976 Pl. 8, Figs. 19-24, Pl. 11, Figs. 4-5

Ceratocyrtis manumi, n. sp.

Ceratocyrtis stoermeri, n. sp.

Challengeron diodon Haeckel, 1887, p. 1654, Pl. 99, Fig. 6; Takahashi and Honjo, 1981, p. 145, Pl. 12, Fig. 3;

Bjørklund, 1974, p. 28-29, Figs. 10a-10i

Clathrospyris sandellae Goll, 1978, p. 179, Pl. 5, Figs. 1-21

Clathrospyris vogti, n. sp.

Corythospyris fiscella Goll, 1978, p. 178, Pl. 5, Figs. 1-21

Corythospyris hispida, $\mathrm{n} . \mathrm{sp}$.

Corythospyris jubata jubata Goll, 1978, p. 177, Pl. 4, Figs. 1, 2, 4, 5, 7-17

Corythospyris jubata sverdrupi, n. subsp.

Corythospyris reuschi, n. sp.

Cycladophora davisiana cornutoides Petrushevskaya, 1967, P. 124, Fig. 70 I-III; Morley, 1980, p. 206, Pl. 1, Figs. 6-10

Cycladophora davisiana davisiana Ehrenberg, 1862, p. 297; 1873, Pl. II, Fig. 11

Cyrtocapsella ampullacea $\mathrm{n} . \mathrm{sp}$.

Cyrtocapsella eldholmi Bjørklund, 1976, p. 1125, Pl. 13, Figs. 12-17

Cyrtocapsella kladaros, $\mathrm{n}$. sp.

Cyrtocapsella japonica (Nakaseko)

Eusyringium japonicum Nakaseko, 1963, p. 193, text-figs. 20-21, Pl. 4, Figs. 1-3

Cyrtocapsella japonica, Nigrini and Lombardi, 1984, p. N107-N108, Pl. 24, Figs. $4 a-4 c$

Cyrtocapsella tetrapera Haeckel, 1887, p. 1512, Pl. 78, Fig. 5

Eucoronis fridtjofnanseni Goll and Bjorklund, 1980, p. 366-370, Pl. 1, Figs. 2-12; Pl. 2, Figs. 1-10; Pl. 3, Figs. 14-26

Eucyrtidium biconicum (Vinassa)

Lithocampe biconica Vinassa, 1900, p. 588, Pl. 3, Fig. 30

Stichopodium biconicum Petrushevskaya and Kozlova, 1979, p. 146, Figs. 406-407

Eucyrtidium inflatum, Kling, 1973, p. 36, Pl. 11, Figs. 7-8, Pl. 15, Figs. 7-10

Eucyrtidium calvertense Martin, 1904, p. 450, Pl. 130, Fig. 5; Hays, 1970, p. 213-214, Pl. 1, Fig. 6; Kling, 1973, p. 636, Pl. 4, Figs. 16, 18,19

Eucyrtidium saccoi (Vinassa)

Stichocorys saccoi Vinassa, 1900, p. 582, Pl. 3, Fig. 17

Stichopodium aff. saccoi, Petrushevskaya and Kozlova, 1979, p. 147, Figs. 408, 529-530

Stichocorys saccoi, Dzinoridze et al. 1978, PI. 39, Figs. 1-3

Euphysetta elegans Borgert, 1902, p. 569, text-Fig. F; Takahashi and Honjo, 1981, p. 156, Pl. 12, Figs. 4-5

Gondwanaria japonica kiaeri, n. subsp.

Hexacontium pachyderma Jørgensen, 1905, p. 115-116, P1. 8, Figs. 31a-31b; Bjørklund, 1976, Pl. 1, Figs. 4-9

Hexalonche esmarki, n. sp.

Hexalonche ?nakasekoi

Hexalonche nakasekoi Petrushevskaya and Kozlova, 1979, p. 90-91, Figs. 206-207

Larcospira bulbosa, n. sp.

Liriospyris cricus Westberg-Smith and Riedel, 1984, p. 491, Pl. 3, Figs. $12 \mathrm{a}-12 \mathrm{c}$

Lophospyris pentagona quadriforis (Haeckel)

Semandrum quadriforis Haeckel, 1887, p. 958, Pl. 92, Fig. 5

Lophospyris pentagona quadriforis, Goll, 1976, p. 398-340, Pl. 13 ,

Figs. 1-13; Pl. 14, Figs. 1-3, 7, 10, 13

Phormospyris thespios, n. sp.

Phorticium clevei (Jørgensen)

Tetrapylonium clevei Jørgensen, 1900, p. 64

Phorticium clevei, Petrushevskaya and Bjørklund, 1974, p. 40, Fig. 7; Bjørklund, 1976, Pl. 4, Figs. 6-10

Pseudodictyophimus amundseni, n. sp.

Pseudodictyophimus gracilipes tetracanthus (Popofsky) 1913

Dictyophimus tetracanthus Popofsky, 1913, p. 333-334, Fig. 42

Pseudodictyophimus gracilipes tetracanthus, Petrushevskaya, 1971, p. 93-95, Fig. 49 I-VII
Pseudodictyophimus horrida (Petrushevskaya)

Corythomelissa horrida Petrushevskaya, 1975, p. 590, Pl. 11, Figs. 14-15; Pl. 21, Fig. 9

Pseudodictyophimus horrida, Petrushevskaya and Kozlova, 1979, p. 120, Figs. 305-306

Pterocanium korotnevi (Dogiel and Reshetnjak)

Pterocorys korotnevi, Dogiel and Reshetnjak, 1952, p. 17, Fig. 11

Pterocanium korotnevi, Lazarus et al., 1985, p. 201-202, Figs. 18. $1-18.5$

Siphocampe arachnea (Ehrenberg)

Lithocampe lineata Ehrenberg, 1838, p. 138

Siphocampe arachnea Nigrini, 1977, p. 255, Pl. 3, Figs. 7-8

Spongaster ?tetras

Spongaster tetras Ehrenberg, 1861, p. 833; 1873b, p. 299, Pl. IV(iii), Fig. 8

Spongocore puella Haeckel 1887, p. 347, Pl. 48, Fig. 6; Nigrini and Moore 1979, p. S 69, Pl. 8, Figs. 5a-5c

Spongotrochus vitabilis, $\mathrm{n}$. $\mathrm{sp}$

Spongurus cauleti, n. sp.

Stichocorys ?delmontensis

Eucyrtidium delmontensis Campbell and Clark, 1944, p. 56, Pl. 7, Figs. 19-20

Stichocorys delmontensis, Nigrini and Lombardi, 1984, p. N 129-N 130, Pl. 25, Fig. 4

Tessarastrum thiedei, $\mathrm{n} . \mathrm{sp}$.

Trisolenia megalactis megalactis Ehrenberg, 1873a, Pl. 8, Fig. 19; 1873b, p. 301; Bjørklund and Goll, 1979, p. 1321-1322, Pl. 5, Figs. 1-21

Velicuccullus oddgurneri Bjørklund, 1976, p. 1126, Pl. 19, Figs. 6-9

Velicuccullus (?) sp. "O", Dzinoridze et al., 1978, Pl. 38, Figs. 1-3; Pl. 40, Fig. 11

\section{SYSTEMATIC DESCRIPTION}

Order SPUMELLARIA Ehrenberg, 1875

Genus ACTINOMMA Haeckel, 1862

Actinomma henningsmoeni $\mathrm{n}$. sp.

(Pl. 2, Figs. 10-15)

Diagnosis. A species of Actinomma characterized by a small, thickwalled cortical shell perforated by small, densely-spaced, round pores, and the presence of 8-10 irregularly distributed internal radial beams that do not penetrate the cortical shell.

Etymology. The species is named in honor of Gunnar Henningsmoen, Professor Emeritus of the University of Oslo.

Description. Skeleton consists of three concentric, approximately spherical lattice shells joined by $8-10$ round radial beams that display no obvious systematic distribution. The diameters of the inner medullary, outer medullary and cortical shells have the approximate ratio of $1: 2: 5$, Internal structure obscured by the massive, relatively smooth cortical shell, which is densely perforated by small round pores. Fine spines project from the cortical shell of rare specimens, but the majority lack such fine spines, and the radial beams never penetrate the cortical shell as major spine processes.

Remarks. Small, more delicately constructed morphs having obvious structural affinities to $A$. henningsmoeni have been observed sporadically in middle and upper Miocene sediments of Site 642.

Occurrences. On the Vøring Plateau Actinomma henningsmoeni ranges from Sections 104-642D-10X-CC to -9X-CC. This species has also been observed in the Cyrtocapsella tetrapera Zone of the central equatorial Pacific (DSDP Site 77).

Size. 110-128 $\mu \mathrm{m}$, maximum diameter of cortical shell.

Holotype. PMO 117.575; Sample 104-642D-10X-6, 25-27 cm; England Finder K13/0; Pl. 2, Figs. 10-11.

\section{Actinomma livae n. sp.}

(Pl. 1, Figs. 1-5)

Diagnosis. A species of Actinomma characterized by a small, sphaerical, well-defined outer medullary shell joined to the large multi-layered cortical shell by numerous stout, bladed radial beams; some of which penetrate the outer cortical shell as short spines.

\section{Et}

Description. Skeleton consists of three or four spherical concentric lattice shells configured as inner and outer medullary shells and one or two cortical shells. The diameters of the three inner shells have an ap- 
proximate ratio of $1: 2: 5$. The structure of the inner medullary shell is obscured by the cortical shells and can not be observed on complete specimens. The outer medullary shell is relatively thick walled and clearly defined as a regular lattice shell, perforated by small, round, densely-spaced pores. On many specimens, fine lattice spines project radially from the outer medullary shell and may have a length approximately one-half the distance to the inner margin of the cortical shell. Additionally, numerous prominent, bladed, irregularly disposed radial beams project from the medullary shell and are joined to the robust, inflated and loosely perforated cortical shell, which may be single or paired. The two cortical shells have similar composition. On specimens with two cortical shells, the major radial beams penetrate the inner cortical shell and are joined to the inner wall of the outer cortical shell. Some of these primary radial beams penetrate the outer cortical shell as short, stout lattice spines. A multitude of secondary radial beams span the space between the cortical shells. The distance between the inner and outer cortical shells varies considerably. The ratio of these two diameters ranges from 1:3 to 1:7.

Remarks. In the development of paired cortical lattice shells, Actinomma livae closely resembles Actinomma holtedahli, from which it is distinguished by the spherical perforate outer medullary lattice shell. Specimens of $A$. holtedahli are characterized by a loosely reticulate outer medullary lattice shell draped between the dense array of delicate radial beams.

Size. 273-296 $\mu \mathrm{m}$, maximum diameter of the outer cortical shell.

Occurrences. Actinomma livae has a sparse occurrence in 104-642D2X-CC and occurs sporadically within the interval from Samples 104642B-25H-1, 75-77 cm to $-1 \mathrm{H}-5,25-26 \mathrm{~cm}$; and from Samples 104$643 \mathrm{~A}-17 \mathrm{H}-1,25-27 \mathrm{~cm}$ to $-16 \mathrm{H}-2,105-107 \mathrm{~cm}$.

Holotype. PMO 117.576; Sample 104-643A-16H-5, 105-107 cm; England finder W30/4; Pl. 1, Figs. 1-3.

\section{Actinomma mirabile n. sp.}

$$
\text { (Pl. 2, Figs. 1-3, Pl. 4, Figs. 33-34) }
$$

Diagnosis. A large species of Actinomma characterized by a delicate, irregularly-shaped outer medullary shell and numerous long, simple spines projecting from the outer cortical shell.

Etymology. The trivial name is derived from the Latin word mirabilis, meaning beautiful.

Description. Skeleton consists of three or four concentric lattice shells interjoined by a dense array of slender, simple, chaotically distributed radial beams. The inner medullary shell is obscured by the external shells, and its structural development has not been observed. The outer medullary lattice shell is small, well-defined, irregular in outline, and composed of a delicate trellise of fine lattice bars. The single or paired cortical lattice shells are massive, spherical, densely perforated by small, round to ovate pores distributed in no obvious geometric pattern. The diameters of the three inner lattice shells have the approximate ratio of $1: 4: 12$. Radial beams penetrate the cortical shell wall and project from the surface as round, simple spines with blunt tips, which may have lengths approximately equal to the inner cortical shell diameter.

Remarks. Short-spined specimens of $A$. mirabile resemble incompletely developed specimens of Actinomma holtedahli, but they are distinguished by the structure of the outer medullary lattice shell.

Occurrences. Actinomma mirabile occurs in the Pseudodictyophimus horrida Zone from Samples 104-642B-20H-7, 24-26 cm to -21H-1, $24-26 \mathrm{~cm}$ and from Samples $104-642 \mathrm{C}-21 \mathrm{H}-4,25-27 \mathrm{~cm}$ to $-22 \mathrm{H}-1,75-$ $77 \mathrm{~cm}$. The species occurs discontinuously from the Ceratocyrtis broeggeri Zone to the $P$. horrida Zone in the interval 104-643A-14H-CC to $12 \mathrm{H}-6,105-107 \mathrm{~cm}$.

Size. 164-181 $\mu \mathrm{m}$, maximum diameter of the inner cortical shell.

Holotype. PMO 117.577; Sample 104-642B-20H-7, 25-27 cm; England Finder M9/2; Pl. 2, Figs. 1-3.

\section{Actinomma plasticum $\mathrm{n} . \mathrm{sp}$.}

$$
\text { (Pl. 2, Figs. 4-9) }
$$

Diagnosis. A species of Actinomma characterized by the presence of 10 or more prominent, tribladed radial beams which project from the outer medullary shell at irregular angles and penetrate the imperfectly spherical cortical shell as stout, bladed lattice spines.

Etymology. The trivial name is derived from the Latin word plasticus, meaning pliable.

Description. Skeleton consists of three concentric lattice shells of irregularly subspherical shape. The inner and outer medullary lattice shells are relatively thick walled, well developed, and clearly distinguishable through the cortical lattice shell. Inner medullary shell is large, approximately spherical and 0.5 times the diameter of the rather loosely trellised outer medullary lattice shell whose outline is distorted from a circular shape at the points of contact with the 10 or more stout, bladed lattice beams projecting from its surface in a chaotic array. The cortical shell is also thick walled, rather loosely trellised by subround to subpolygonal pores, and ranges in shape from irregularly sphaerical to broadly undulatory. The maximum diameter of the three lattice shells has the ratio of $1: 2: 5$. The internal radial beams project through the cortical shell as prominent, bladed, irregularly distributed lattice spines ranging in length up to 0.5 times the diameter of the cortical shell.

Remarks. Actinomma plasticum has a superficial resemblance to Hexalonche esmarki, but it differs from the latter by the presence of three lattice shells, smaller overall size, and the less robust but more imperfectly spherical cortical shell.

Occurrences. Actinomma plasticum occurs in the A. plasticum Zone to $P$. horrida Zone of Samples 104-642B-22H-1, 75-77 cm to -20H-6, 24-26 cm and discontinuously over a broader range in Samples 104$642 \mathrm{C}-24 \mathrm{H}-3,25-27 \mathrm{~cm}$ to $-18 \mathrm{H}-4,25-27 \mathrm{~cm}$. The species also occurs in the $P$. horrida Zone of Samples 104-643A-13H-3, 104-106 cm to $12 \mathrm{H}-4$, $105-107 \mathrm{~cm}$.

Size. 135-156 $\mu \mathrm{m}$, maximum diameter of the cortical shell.

Holotype. PMO 117.578; Sample 104-643A-12H-4, 105-107 cm; England Finder R10/3; Pl. 2, Figs. 4-6.

\section{Genus HEXALONCHE Haeckel , 1881 \\ Hexalonche esmarki $\mathrm{n}$. sp. \\ (PI. 1, Figs. 6-10)}

Diagnosis. A species of Hexalonche characterized by its large size, massive large-pored cortical shell, and numerous prominent tribladed internal radial beams.

Etymology. The species is named posthumously in honor of Prof. Jens E. Esmark, 1763-1839, Royal Frederick's University, Oslo.

Description. Skeleton consists of two concentric spherical lattice shells. Medullary lattice shell is small, delicate, densely perforated by fine round lattice pores. Numerous stout, tribladed, irregularly-disposed radial beams interjoin the two lattice shells and penetrate the cortical shell wall as short simple spines lacking blades. The cortical shell is very thick-walled and sparsely perforated by irregular, round to subpolygonal lattice spines. Cortical lattice bars are broadly sulcate on the external margin and may be finely spinous.

Remarks. Hexalonche esmarki is distinguished from Hexalonche ?nakasekoi by its larger overall size and large concentric medullary lattice shell.

Occurrences. Hexalonche esmarki occurs exclusively in the $H$. esmarki Zone in Samples 104-642B-15H-2, 27-29 cm to -15H-1, 27-29 cm and in Samples 104-642C-16H-3, 25-27 cm to $-16 \mathrm{H}, 25-27 \mathrm{~cm}$. The species also occurs in Section 104-643A-9YH, CC to Sample -8-4, 21-23 cm.

Size. 167-195 $\mu \mathrm{m}$, maximum diameter of the cortical shell.

Holotype. PMO 117.579; Sample 104-642B-15H-2, 27-29 cm; England Finder P40/0; Pl. 1, Figs. 7-9.

\section{Genus LARCOSPIRA Haeckel, 1887 Larcospira bulbosa, n. sp.} (Pl. 2, Figs. 16-21)

Diagnosis. A species of Larcospira characterized by its thick-walled, uniformly perforate cortical lattice shell and irregularly swollen or invaginated margin.

Etymology. The trivial name is derived from the Latin word bulbosus, meaning swelling.

Description. The skeleton consists of three lattice whorls that appear as simple lattice shells in plane view. The whorls are interjoined by a multitude of delicate radial beams that do not penetrate the cortical shell as spines. Cortical shell thick walled, densely and uniformly perforated by small, round pores. Medullary shells ovate in outline. Cortical shell margin varies from smoothly ovate to laterally constricted with alternating swellings and invaginations.

Occurrence. Larcospira bulbosa occurs only in Section 104-642C$15 \mathrm{H}, \mathrm{CC}$ to Sample $-15 \mathrm{H}-7,25-27 \mathrm{~cm}$.

Size. 116-145 $\mu \mathrm{m}$, maximum diameter of the cortical lattice shell.

Holotype. PMO 117.580; Section 104-642C-15H, CC; England Finder W35/3; Pl. 2, Figs. 16-19. 


\section{Genus SPONGOTROCHUS Haeckel, 1861}

Spongotrochus vitabilis, n. sp.

$$
\text { (Pl. 3, Figs. 1-3) }
$$

Diagnosis. A species of Spongotrochus characterized by its loosely trellised and scalloped marginal lattice shell, from which project a chaotic array of radial spines.

Etymology. The trivial name is the Latin word vitabilis, meaning avoidable.

Description. Spongodiscid skeleton configured as a flat circular disk. Central region consists of delicate but densely distributed spongy lattice. Toward the margin, the lattice becomes more robust and more loosely trellised. Numerous chaotically disposed radial beams project through the spongy lattice as prominent spines that may be hollow or solid and round or sulcate.

Occurrence. Rare or trace specimens of Spongotrochus vitabilis have been found in Samples 104-642B-23H-1, 75-77 cm, -642C-24H-4, 25-27 $\mathrm{cm}$, and $642 \mathrm{C}-22 \mathrm{H}-1,75-77 \mathrm{~cm}$, but these occurrences are regarded as having resulted from sediment reworking or laboratory contamination. The species is thought to be restricted to the $S$. vitabilis Zone and lower Cycladophora davisiana cornutoides Zone sediments in Samples 104643A-20X-5, 105-107 cm to $-19 \mathrm{X}-2,105-107 \mathrm{~cm}$.

Size. 200-274 $\mu$ m, maximum diameter of the cortical shell.

Holotype. PMO 117.581; Sample 104-643A-20X-3, 105-107 cm; England Finder T46/4; Pl. 3, Fig. 1.

\section{Genus SPONGURUS Haeckel, 1862 \\ Spongurus cauleti $\mathrm{n}$. sp. \\ (Pl. 3, Figs. 4-9)}

Diagnosis. Elongate ellipsoidal skeleton composed of thin-walled lattice arranged in loose whorls, through which project polar lattice spines.

Etymology. The species is named in honor of our colleague, Dr. Jean-Pierre Caulet, National Museum of Natural History, Paris, France.

Description. The skeleton consists of delicate lattice organized in loose, irregular whorls that become progressively more widely spaced. Individual whorls rarely extend continuously from the center to the outer margin but terminate and coalesce with other whorls in a complex manner. Numerous delicate perpendicular beams interjoin whorl layers. Overall shape imperfectly ellipsoidal, with the longest axis approximately twice the width. Three to five whorls compose the central region. Two to three additional whorl segments (caps) are present in the polar regions. Lattice is densely perforated by small round pores of uniform size. On completely developed specimens, one to five stout lattice bars radiate from the central region and penetrate the consecutive whorls to project from the poles as prominent spines.

Remarks. This species is only provisionally assigned to Spongurus, as taxonomic revision at the generic level is required. Spongurus cauleti bears a superficial resemblance to Tessarastrum thiedei, from which it is distinguished by its delicate whorl construction and polar spines.

Occurrence. Spongurus cauleti has continuous occurrences in the $S$. cauleti Zone and lower $H$. esmarki Zone of: Samples 104-642B-16H-2, $31-33 \mathrm{~cm}$ to $-15 \mathrm{H}-3,27-29 \mathrm{~cm} ; 104-642 \mathrm{C}-16 \mathrm{H}-5,25-27 \mathrm{~cm}$ to $-16 \mathrm{H}-3$, $25-27 \mathrm{~cm}$; and $104-643 \mathrm{~A}-1 \mathrm{OH}-5,105-107 \mathrm{~cm}$ to $-9 \mathrm{H}-2,105-107 \mathrm{~cm}$

Size. $205-310 \mu \mathrm{m}$, maximum longitudinal dimension of lattice whorls.

Holotype. PMO 117.582; 104-642B-16H-2, 31-33 cm; England Finder D16/1; Pl. 3, Figs. 4-5.

\section{Genus TESSARASTRUM Haeckel, 1887}

Tessarastrum thiedei $\mathrm{n} . \mathrm{sp}$.

$$
\text { (Pl. 3, Figs. 10-17) }
$$

Diagnosis. A species of Tessarastrum characterized by its thickwalled lattice shells and elongate outline.

Etymology. The species is named in honor of Professor Jørn Thiede, University of Kiel, Federal Republic of Germany.

Description. Skeleton consists of thick-walled, somewhat discoidal concentric lattice shells and shell segments that are closely spaced and densely perforated by round pores of uniform size. The outline of the skeleton is pronouncedly elongate, with the longitudinal dimension three to four times the maximum width. The proximate portion of the skeleton is composed of two to three medullary lattice shells. Three to six polar caps are present on the longitudinal axis and progressively increase slightly in width distally to form two broad polar processes. A single- layered, delicate patagial girdle surrounds the central region of many specimens. Completely developed specimens may have one to three short slender spines projecting from a weakly developed pylome at one pole.

Occurrence. Tessarastrum thiedei is consistently present and frequently dominant in the $T$. thiedei Zone to the basal Antarctissa whitei Zone in Samples 104-642B-15H-1, 27-29 cm to $-12 \mathrm{H}-3,25-27 \mathrm{~cm}$; and $104-642 \mathrm{C}-15 \mathrm{H}-6,75-77 \mathrm{~cm}$ to Section $104-642 \mathrm{C}-12 \mathrm{H}, \mathrm{CC}$.

Size. 205-265 $\mu \mathrm{m}$, maximum longitudinal dimension.

Holotype. PMO 117.583; Sample 104-642B-13H-5, 24-26 cm; England Finder Z8/3; Pl. 3, Figs. 10-11.

\section{Order NASSELLARIA Ehrenberg, 1875 \\ Genus CERATOCYRTIS Bütschli, 1882; emended Petrushevskaya, 1981}

Ceratocyrtis broeggeri $\mathrm{n} . \mathrm{sp}$.

$$
\text { (Pl. 5, Figs. 14-18) }
$$

Ceratocyrtis aucullaris f. goetheana Petrushevskaya and Kozlova 1979, p. 112 , Fig. 294

Diagnosis. A species of Ceratocyrtis characterized by broad smooth lattice bars and large irregular pores on the median portion of the thorax.

Etymology. The species is named posthumously in honor of Professor Waldemar C. Brøgger, 1851-1933, Royal Frederick's University, Oslo.

Description. Skeleton consists of two heavy-walled, sparsely-trellised lattice chambers. The cephalis is small, perforated by large open pores, and partially submerged in the broad, tubular thorax. Cephalis bears short external spines and a prominent axial spine projects into the thoraxic cavity. Upper margin of thorax swells sharply, and irregularly disposed short spines project upward from its surface. Thoracic lattice pores of irregular shape and distribution become increasingly large in the mid-length region. On many specimens, the most distal rows of lattice pores are of reduced size above the straight terminal margin, which is commonly inverted and partially enclosed.

Remarks. We have not been able to find in our sample from DSDP, 38-338-10-2, specimens similar to that illustrated by Petrushevskaya and Kozlova (1979).

Occurrences. Ceratocyrtis broeggeri occurs only in the $C$. broeggeri Zone of Samples 104-643A-15H-4, 25-27 cm to $-14 \mathrm{H}-1,109-111 \mathrm{~cm}$; Samples 104-642B-22H-6, 75-77 cm; Sample 104-642C-24H-3, 25-27 $\mathrm{cm}$; and Sample 104-642D-2X-6, 25-27 cm.

Size. 138-162 $\mu \mathrm{m}$, maximum height of total lattice shell; 123-128 $\mu \mathrm{m}$, maximum thoracic width.

Holotype. PMO 117.584; Sample 104-643A-15H-3, 107-109 cm; England Finder G54/2; Pl. 5, Figs. 14-16.

\section{Ceratocyrtis manumi $\mathrm{n} . \mathrm{sp}$}

(Pl. 5, Figs. 21-23)

Diagnosis. A species of Ceratocyrtis characterized by a broad, lowdomed cephalis and an hour-glass shaped thorax.

Etymology. The species is named in honor of Professor Svein Manum, University of Oslo.

Description. The skeleton consists of two moderately thin-walled and uniformly perforated lattice chambers. The densely perforated cephalis is broad, low-domed and not significantly recessed within the thoracic chamber. Numerous short spines project upwardly from the cephalis. Two larger spines are the A- and V-processes. Only a slight constriction separates the cephalis and thorax. Upper margin of the thorax is conical, swells broadly, and is chaotically spinous. Three prominent lattice processes originate at the LL and D bars, are joined to the upper thorax, and project laterally from its margin as free spines on some specimens. The long thorax is horizontally constricted at mid-length and swells again distally above an irregular terminal margin.

Remarks. Ceratocyrtis manumi is distinguished from Gondwanaria nigrinae by its more loosely trellised lattice composition and significantly different cephalic morphology.

Occurrences. Ceratocyrtis manumi has a trace occurrence in Section $104-642 \mathrm{~B}-22 \mathrm{H}, \mathrm{CC}$ and is consistently present in Section 104-643A$17 \mathrm{H}, \mathrm{CC}$ to Sample $15 \mathrm{H}-5,105-107 \mathrm{~cm}$.

Size. 126-148 $\mu \mathrm{m}$, maximum height; $122-130 \mu \mathrm{m}$, maximum thoracic width

Holotype. PMO 117.585; Sample 104-643A-16H-3, 105-107 cm; England Finder X25/4; Pl. 5, Figs. 21-23. 
Ceratocyrtis stoermeri n. sp.

(PI. 5, Figs. 5-9)

Diagnosis. A species of Ceratocyrtis characterized by its massive cyclindrical thorax with prominent lattice bar ridges forming polygonal pore frames.

Etymology. The species is named posthumously in honor of Professor Leif Størmer, 1905-1979, University of Oslo.

Description. The skeleton consists of two thick-walled lattice chambers. Cephalis is small, porous, densely spinous, and partially recessed in the thoracic cavity. Upper surface of massive thorax swells gradually, is densely spininous, and perforated by small round pores. Maximum lattice shell width is at the mid-length of the thorax, where the irregularly arranged pores are also largest. Below this point, the thorax tapers slightly, and the distal termination is straight, with a sieve plate covering the basal aperture on some specimens. The external surfaces of the thoracic lattice bars bear broad ridges which form polygonal pore frames for the larger pores.

Remarks. Specimens of Ceratocyrtis mashae have more loosely trellised and less massive thoracic composition.

Occurrences. Ceratocyrtis stoermeri occurs in the $C$. broeggeri Zone of: Section 104-642B-22H, CC to Sample 104-642B-22H-6, 75-77 cm; Samples $104-642 \mathrm{C}-24 \mathrm{H}-4,25-27 \mathrm{~cm}$ to $-24 \mathrm{H}-3,25-27 \mathrm{~cm}$; and $104-$ 642D-2X-6, 25-27 cm.

Size. $156-167 \mu \mathrm{m}$, maximum lattice height; $128-145 \mu \mathrm{m}$, maximum thoracic width.

Holotype. PMO 117.586; Sample 104-642B-23X-1, 25-27 cm; England Finder T49/0; Pl. 5, Figs. 5-7.

\section{Genus CLATHROSPYRIS Haeckel, 1881}

\section{Clathrospyris vogti $\mathrm{n} . \mathrm{sp}$.}

(Pl. 4, Figs. 25, 26, 32)

Diagnosis. A species of Clathrospyris characterized by the presence of a single pair of sagittal lattice pores on the D-side of the cephalis.

Etymology. The species is named posthumously in honor of Professor Johan H. L. Vogt, 1858-1932, Royal Frederick's University, Oslo.

Description. Skeleton consists of a simple cephalis on many specimens. Some specimens have additionally a short irregular thorax joined to the three basal spines. Cephalis large, thick-walled, smooth or chaotically spinous, joined directly to the top of the sagittal ring. The loose trellise of cephalic lattice is confined to lateral margins and V-side of cephalis and frames a single large pair of sagittal lattice pores on the Dside of the cephalis. A- and V-spines short. Three spines project basally as extensions of the D- and LL bars. Some specimens have additional irregularly disposed basal spines. Rare specimens have a short skirt of loosely trellised thoracic lattice joined to the basal spines.

Remarks. Clathrospyris vogti includes specimens described by Goll and Bjørklund (1980), as transitional between Clathrospyris sandellae and Eucoronis fridtjofnanseni.

Occurrence. Clathrospyris vogti is restricted to the $C$. vogti Zone in Sample $104-643 \mathrm{~A}-12 \mathrm{H}-2,105-107 \mathrm{~cm}$ to $-12 \mathrm{H}-1,105-107 \mathrm{~cm}$.

Size. $105-135 \mu \mathrm{m}$, maximum cephalic width

Holotype. PMO 117.587; Sample 104-643A-12H-1, 27-29 cm; England Finder E54/0; Pl. 4, Fig. 32.

\section{Genus CORYTHOSPYRIS Haeckel, 1881 \\ Corythospyris hispida $\mathrm{n} . \mathrm{sp}$. (Pl. 4, Figs. 17-22)}

Diagnosis. A species of Corythospyris characterized by an enlarged pair of sagittal lattice pores on the D-side of the irregularly spinous cephalis. hairy.

Etymology. The trivial name is the Latin word hispida, meaning

Description. The skeleton consists solely of a small, thin-walled cephalis. Lattice bands, bearing very fine irregularly disposed spines and perforated by small round pores, arch between the lateral margins of the basal ring and the top of the sagittal ring and frame-enlarged sagittal lattice pores on the D- and V-sides. A-spine prominent. V-spine extremely small. Three short basal spines project laterally from the D- and LL-bars.

Remarks. Corythospyris hispida differs from Corythospyris reuschi in its smaller size, the presence of chaotic lattice spines, and the absence of a lattice bar arching across the D-side of the cephalis.
Occurrences. Corythospyris hispida occurs in the Cyrtocapsella eldholmi Zone and Pseudodictyophimus horrida Zone in: Section 104642B-21H, CC to Sample 104-642B-20H-2, 24-26 cm; and Samples $104-$ $642 \mathrm{C}-23 \mathrm{H}-5,24-26 \mathrm{~cm}$ to $-20 \mathrm{H}-2,25-27 \mathrm{~cm}$. The species also ranges discontinuously in Samples 104-643A-26X-6, 105-107 cm to $-12 \mathrm{H}-1$, $105-107 \mathrm{~cm}$.

Size. 72-85 $\mu \mathrm{m}$, maximum cephalic width.

Holotype. PMO 117.588; 104-642C-23H-3, 24-27 cm; England Finder T49/1; Pl. 4, Figs. 17-19.

\section{Corythospyris jubata Goll, 1978}

Corythospyris jubata sverdrupi $\mathrm{n}$. subsp.

(PI. 4, Figs. 1-8)

Diagnosis. A subspecies of $C$. jubata characterized by the presence of numerous small tubercles on the surface of the cephalis.

Etymology. The species is named posthumously in honor of Harald U. Sverdrup, 1888-1957, Museum of Bergen.

Description. The skeleton consists solely of a simple, moderately thick-walled cephalis, which is densely perforated by small round pores of approximately equal size. Individual lattice bars have no exterior ridges or prominent lattice spines characteristic of $C$. jubata jubata. Junctures between lattice bars bear broad swellings, which are finely spinous on some specimens. Three basal spines may be short and simple or long with chaotic distal bifurcations.

Remarks. This morphology represents the final stage of the Corythospyris jubata lineage.

Occurrences. Corythospyris jubata sverdrupi ranges from the $C$. jubata sverdrupi Zone to the Cycladophora davisiana cornutoides Zone in Samples 104-643A-25X-3, 105-107 cm to $19 \mathrm{X}-2,105-107 \mathrm{~cm}$. The species also occur in Sample 104-642B-24H-5, 75-77 cm to -23H, CC, and Samples $104-642 \mathrm{D}-4 \mathrm{X}-6,24-26 \mathrm{~cm}$ to $-3 \mathrm{X}-4,24-26 \mathrm{~cm}$.

Size. $75-92 \mu \mathrm{m}$, maximum cephalic width.

Holotype. PMO 117.589; Sample 104-643A-24X-3, 105-107 cm; England Finder B20/3; Pl. 4, Figs. 1-2.

\section{Corythospyris reuschi $\mathrm{n} . \mathrm{sp}$.}

$$
\text { (PI. 4, Figs. 9-16) }
$$

Diagnosis. A large species of Corythospyris characterized by a long single lattice bar that spans the D-side of the cephalis.

Etymology. The species is named posthumously in honor of Hans H. Reusch, 1852-1922, Director of the Geological Survey of Norway.

Description. Skeleton consists solely of a small, smooth, thin-walled cephalis perforated by pores of differing size. Pores of the lateral margins and V-side are small and densely spaced. The D-side of the cephalis bears a large pair of sagittal lattice pores that are partially obscured by a long arching lattice bar spanning the width of the cephalis. A-spine prominent; $\mathrm{V}$-spine very small. Three short basal spines project laterally as extensions of the D- and LL-bars.

Remarks. Corythospyris reuschi is distinguished from Corythospyris fiscella by the presence of enlarged sagittal lattice pores.

Occurrences. Corythospyris reuschi occurs in the Eucoronis fridtjofnanseni/C. reuschi Zone to the Tessarastrum thiedei Zone in: Samples $104-642 \mathrm{~B}-18 \mathrm{H}-4,24-26 \mathrm{~cm}$ to $-14 \mathrm{H}-1,35-37 \mathrm{~cm} ; 104-642 \mathrm{C}-19 \mathrm{H}-2,25-$ $27 \mathrm{~cm}$ to $16 \mathrm{H}-6,25-27 \mathrm{~cm}$; and 104-642A-11H, CC to $9 \mathrm{H}-4,105-107 \mathrm{~cm}$.

Size. $72-85 \mu \mathrm{m}$, maximum cephalic width.

Holotype. PMO 117.590; Sample 104-642C-17H-2, 25-27 cm; England Finder N48/2; Pl. 4, Figs. 9-11.

\section{Genus CYRTOCAPSELLA Haeckel, 1887 \\ Cyrtocapsella kladaros $\mathrm{n}$. $\mathrm{sp}$. \\ (PI. 5, Figs. 24-26)}

Cyrtocapsella sp. Bjørklund 1976, Pl. 17, Fig. 14.

Diagnosis. A species of Cyrtocapsella characterized by a thin-walled inflated skeleton with densely spaced lattice pores on the three post-cephalic chambers. frail.

Etymology. The trivial name is the Greek word kladaros, meaning

Description. The skeleton consists of four thin-walled lattice chambers. The small, smooth and imperforate cephalis projects well above the thorax, from which it is separated by a distinctive horizontal stricture. Well-developed internal and external strictures also separate the 
three inflated post-cephalic chambers, of which the abdomen is largest. The fourth chamber is closed and bowl-shaped. These three chambers are densely perforated by small round pores of approximately equal size, which may be arranged in imperfect rows. Lattice bars are slender and impart a delicate aspect to the skeleton.

Remarks. The thin-walled, densely-perforate nature of Cyrtocapsella kladaros distinguishes it from other Cyrtocapsella species. Many specimens appear somewhat distorted and strictures are often oblique.

Occurrences. This short-ranging species is confined to: Samples 104642B-22H-5, 75-77 cm to $-22 \mathrm{H}-3,75-77 \mathrm{~cm} ; 104-642 \mathrm{C}-24 \mathrm{H}-2,25-27$ cm to $-24 \mathrm{H}-1,25-27 \mathrm{~cm}$; 104-642D-2X-5, 25-27 cm to $-2 \mathrm{X}-2,25-27 \mathrm{~cm}$; and Section 104-643A-13H, CC to Sample 104-643A-13H-3, 104-106 $\mathrm{cm}$.

Size. 145-190 $\mu \mathrm{m}$, maximum height; $112-148 \mu \mathrm{m}$, maximum abdominal width.

Holotype. PMO 117.591; Sample 104-643A-13H-5, 107-109 cm; England Finder P54/0; Pl. 5, Figs. 24-26.

\section{Cyrtocapsella ampullacea $\mathrm{n} . \mathrm{sp}$.}

(Pl. 5, Figs. 10-13, 19, 20)

Diagnosis. A species of Cyrtocapsella characterized by its two small, thin-walled post-cephalic chambers.

Etymology. The trivial name is the Latin word ampullaceus, meaning bottle-like.

Description. The skeleton consists of three chambers, which increase gradually in size. The small, smooth cephalis is well exposed above the thorax. The thin-walled and densely perforate thorax and abdomen have uniform compositions and are separated by a pronounced stricture. The abdomen is inflated, enclosed, and recurves distally in characteristic outline on many specimens. The thorax and abdomen are approximately equidimensional on other specimens.

Remarks. This species may be an evolutionary derivative of Cyrtocapsella kladaros, although intermediates have not been observed.

Occurrences. This short-ranging species is confined to: Samples 104$642 \mathrm{~B}-22 \mathrm{H}-3,75-77 \mathrm{~cm}$ to $-22 \mathrm{H}-2,75-77 \mathrm{~cm}$; and Section 104-642C$23 \mathrm{H}, \mathrm{CC}$ to Sample $-23 \mathrm{H}-3,24-26 \mathrm{~cm}$.

Size. 108-124 $\mu \mathrm{m}$, maximum height of total lattice shell.

Holotype. PMO 117.592; Section 104-642C-22H, CC; England Finder E24/4; Pl. 5, Figs. 10-11.

\section{Genus GONDWANARIA Petrushevskaya, 1975 \\ Gondwanaria japonica (Nakaseko, 1963) \\ Gondwanaria japonica kiaeri $\mathrm{n}$. sp.}

(Pl. 4, Figs. 27-28)

Diagnosis. A subspecies of $G$. japonica characterized by the presence of a long stout A-spine.

Etymology. The species is named posthumously in honor of Professor Johan A. Kiaer, 1869-1931, Royal Frederick's University, Oslo.

Description. A-spine stout, one to four times longer than the diameter of the cephalis.

Occurrences. G. japonica kiaeri occurs in: Samples 104-642D-8X-1, $25-27 \mathrm{~cm}$ to $-6 \mathrm{X}-1,30-32 \mathrm{~cm}$; and Section 104-643A-29X, CC to Sample 104-643A-26X-5, 105-107 cm.
Size. $80-90 \mu \mathrm{m}$, maximum thoracic width.

Holotype. PMO 117.593; Sample 104-643A-29X-5, 105-107 cm; England Finder Z23/4; Pl. 5, Figs. 27-28.

\section{Genus PHORMOSPYRIS Haeckel, 1881}

Phormospyris thespios $\mathrm{n} . \mathrm{sp}$.

(PI. 4, Figs. 23-24, 29-31)

Diagnosis. A species of Phormospyris characterized by the large, thin-walled cephalis which encloses the sagittal ring.

Etymology. The trivial name is the Greek word thespios, meaning divine or wondrous.

Description. The skeleton consists solely of the large, smooth cephalis and short irregular thorax. The cephalis is thin-walled, densely perforated by small round pores of approximately equal size, and it arches across the top of the sagittal ring. The A- and V-spines are short. Three prominent basal spines project downward from the basal ring as projections of the D- and LL-bars. A short skirt of irregular thoracic lattice is joined to the basal spines and frames a large basal aperture.

Remarks. Phormospyris thespios appears to be the northern member of a bipolar species-pair with Phormospyris antarctica as the southern counterpart.

Occurrence. Phormospyris thespios occurs in: Samples 104-642B$15 \mathrm{H}-1,27-29 \mathrm{~cm}$ to $-14 \mathrm{H}, \mathrm{CC}$; $104-642 \mathrm{C}-15 \mathrm{H}, \mathrm{CC}$ to $15 \mathrm{H}-4,25-27 \mathrm{~cm}$; and $104-643 \mathrm{~A}-8 \mathrm{H}-5,103-105 \mathrm{~cm}$

Size. $112-138 \mu \mathrm{m}$, maximum cephalic width.

Holotype. PMO 117.594; Sample 104-642C-15H-6, 25-27 cm; England Finder D29/0; Pl. 4, Figs. 23-24, 29-30.

Genus PSEUDODICTYOPHIMUS Petrushevskaya, 1971

\section{Pseudodictyophimus amundseni $\mathrm{n} . \mathrm{sp}$.}

(Pl. 5, Figs. 1-4)

Diagnosis. A species of Pseudodictyophimus characterized by its large size and inflated, loosely trellised cephalis.

Etymology. The species is named posthumously in honor of Roald Amundsen, 1872-1928, Norwegian polar explorer.

Description. Four large cruciform spines project radially as extensions of the cephalic elements: the A-, D- and LL-bars. The large, thickwalled cephalis is perforated by large lattice pores, spans the proximal portions of these spines, and extends basally as an irregular thorax with a wide basal aperture. A prominent V-spine has similar morphology to the primary tetrad and is approximately half their length.

Remarks. Its large size and heavy, porous cephalis distinguishes $P$. ammundseni from other numerous Neogene Pseudodictyophimus species (mostly undescribed) in Leg 104 sediments.

Occurrence. Pseudodictyophimus amundseni occurs in: Section 104$642 \mathrm{~B}-23 \mathrm{H}, \mathrm{CC}$ to $-23 \mathrm{H}-2,75-77 \mathrm{~cm} ; 104-642 \mathrm{C}-24 \mathrm{H}, \mathrm{CC}$ to $-24 \mathrm{H}-3,28$ $27 \mathrm{~cm} ; 104-642 \mathrm{D}-3 \mathrm{X}, \mathrm{CC}$ to $-3 \mathrm{X}-2,25-27 \mathrm{~cm}$; and 104-643A-23-4, $105-$ $107 \mathrm{~cm}$ to $21-1,105-107 \mathrm{~cm}$.

Size. 64-75 $\mu \mathrm{m}$, maximum cephalic width.

Holotype. PMO 117.595; Section 104-643A-21X, CC; England Finder V23/1; Pl. 5, Figs. 1-3. 


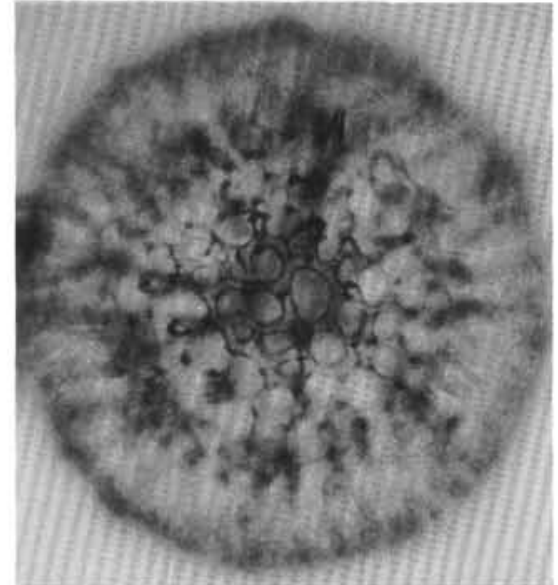

1

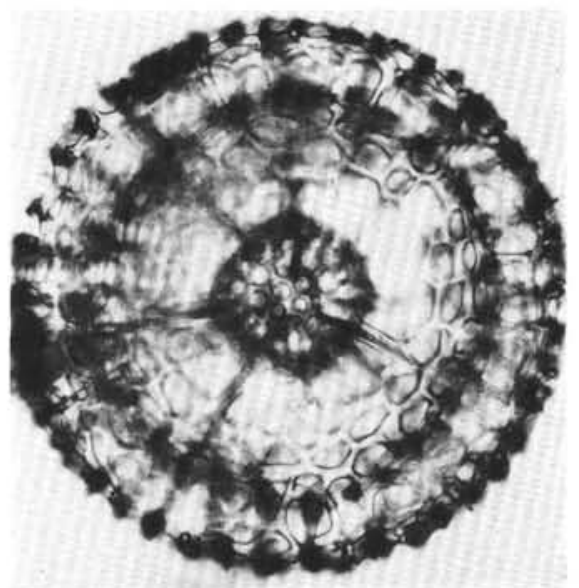

4

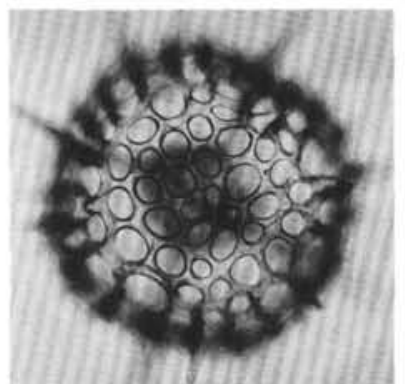

7

8

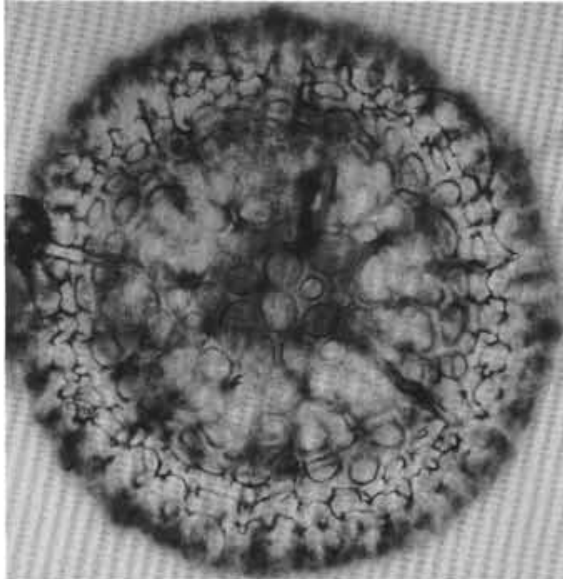

2

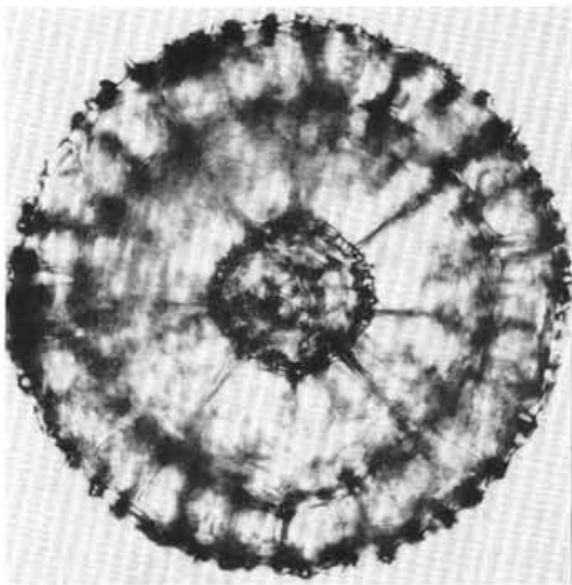

5

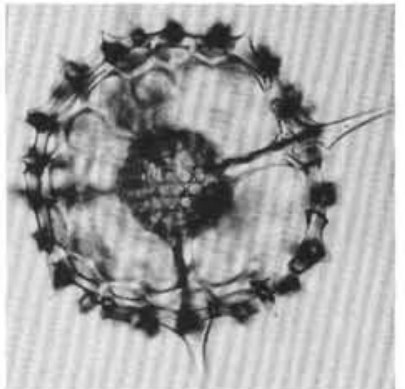

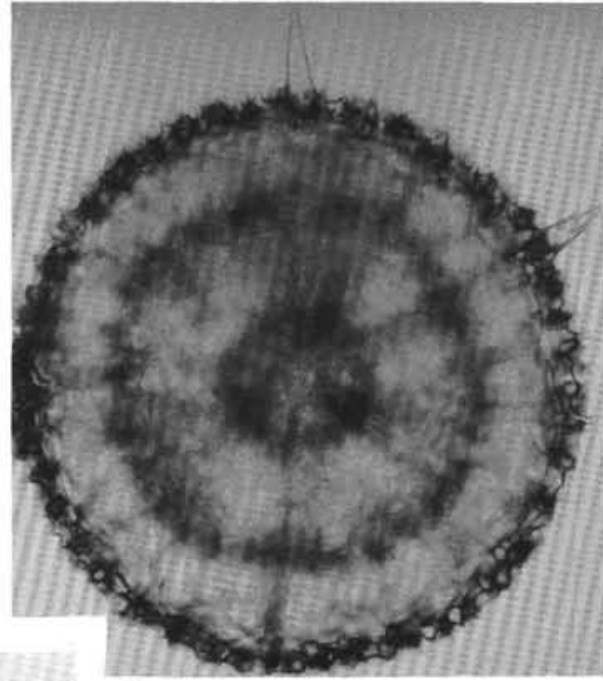

3

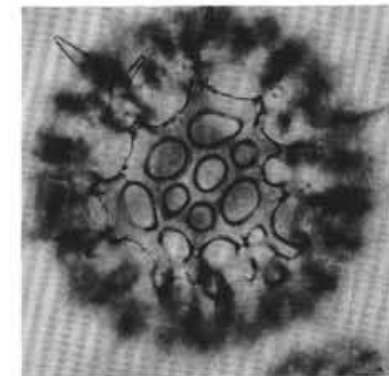

6

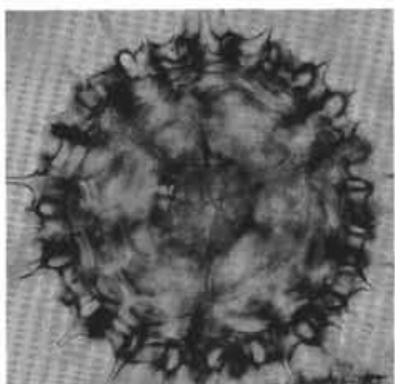

10

Plate 1. Scale bar $=100 \mu \mathrm{m}$. Figures 1-5. Actinomma livae n. sp. 1-3. Holotype; PMO 117.576; England Finder W30/4; Sample 104-643A$16 \mathrm{H}-5,105-107 \mathrm{~cm}$. 4, 5. Sample 104-642D-2X-7, 25-27 cm. Figures 6-10. Hexalonche esmarki n. sp. 7-9. Holotype; PMO 117.579; England Finder P40/0; Sample 104-642B-15H-2, 27-29 cm. 6, 10. Sample 104-642C-16H-3, 71-73 cm. 


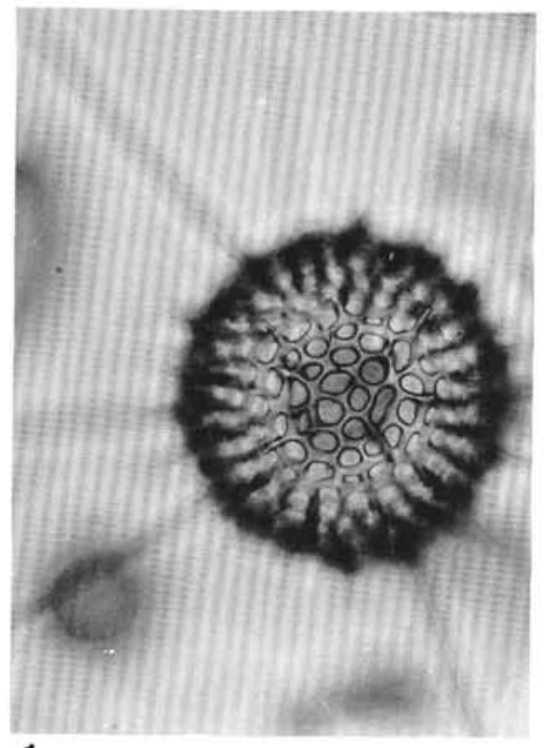

1

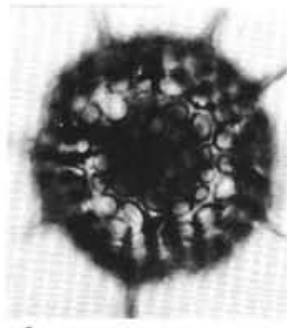

4

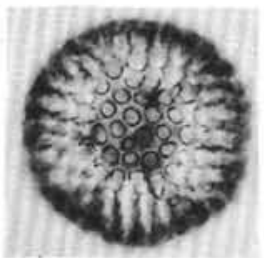

10

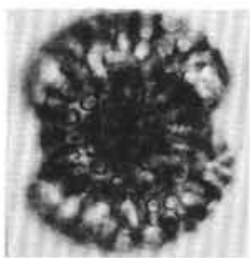

16

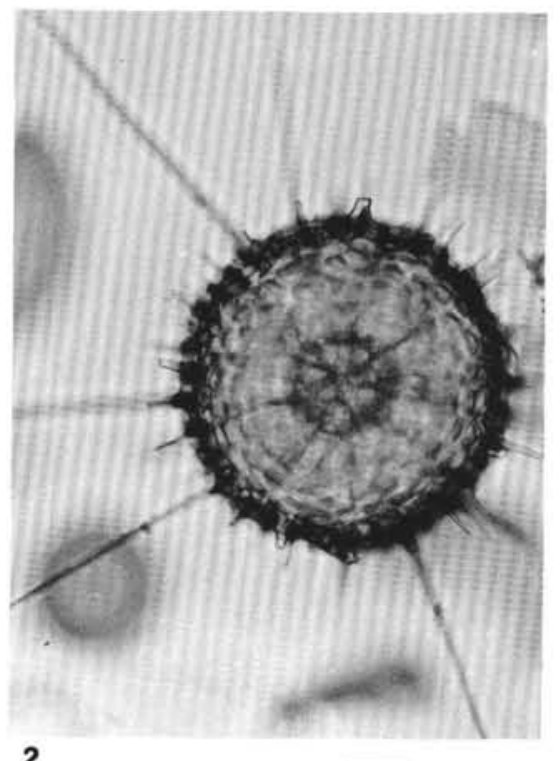

2

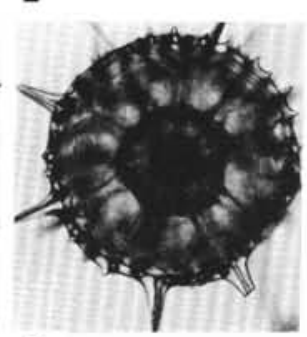

6

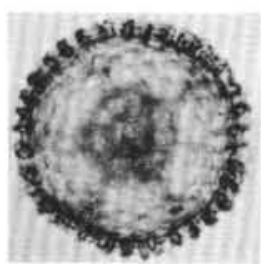

11

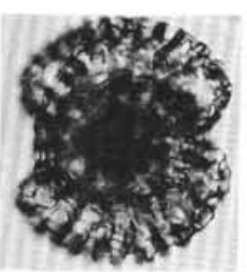

17

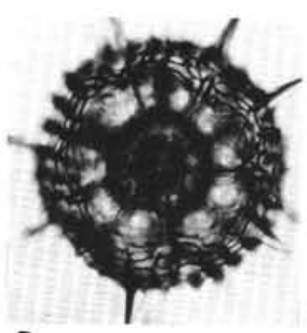

5

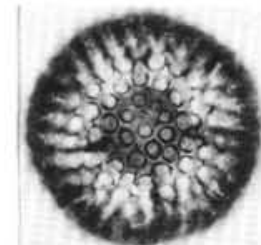

12

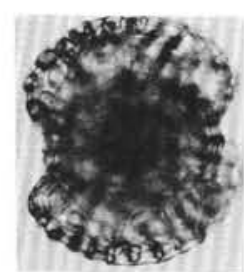

18

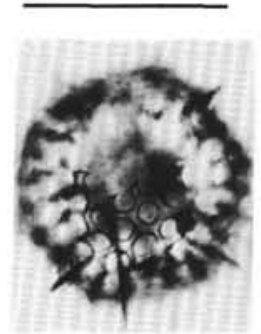

7

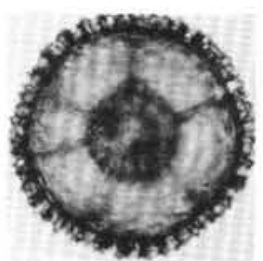

13

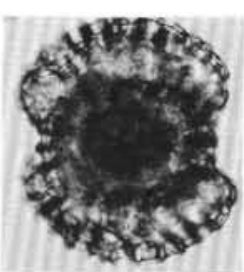

19

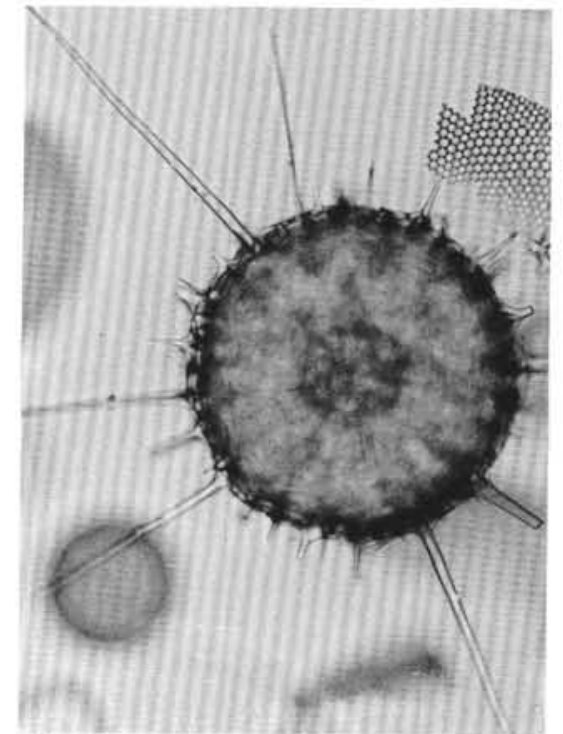

3

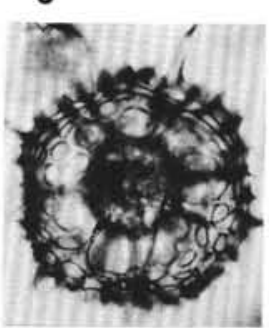

8

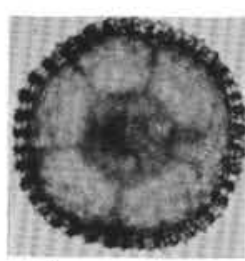

14

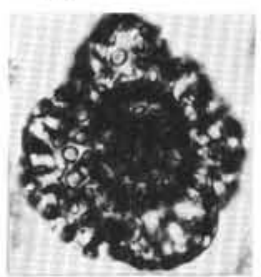

20

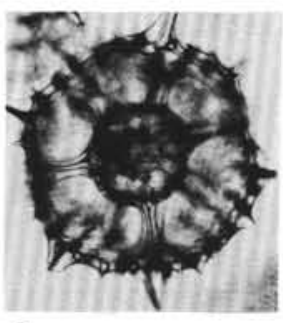

9

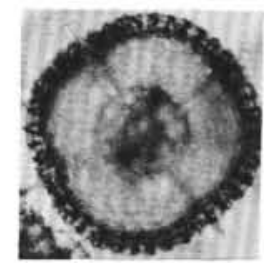

15

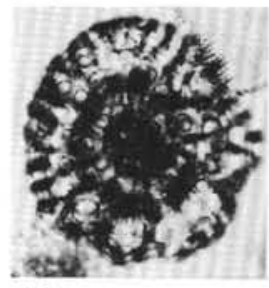

21

Plate 2. Scale bar $=100 \mu \mathrm{m}$. Figures 1-3. Actinomma mirabile n. sp., holotype; PMO 117.577; England Finder M9/2; Sample 104-642B-20H-7, 25-27 cm. Figures 4-9. Actinomma plasticum n. sp. 4-6. Holotype; PMO 117.578; England Finder R10/3; Sample 104-643A-12H-4, 105-107 $\mathrm{cm}$. 7-9. England Finder T42/4; Sample 104-643A-12H-4, 105-107 cm. Figures 10-15. Actinomma henningsmoeni n. sp. 10, 11. Holotype; PMO 117.575; England Finder K13/0; Sample 104-642D-10X-6, 25-27 cm. 12-15. Samples 104-642D-10X-3, 25-28 cm. 12, 13. Same specimen. Figures 16-21. Larcospira bulbosa n. sp. 16-19. Holotype; PMO 117.580; England Finder W35/3; Section 104-642C-15H, CC. 20-21. Section 104-642D-15X, CC. 

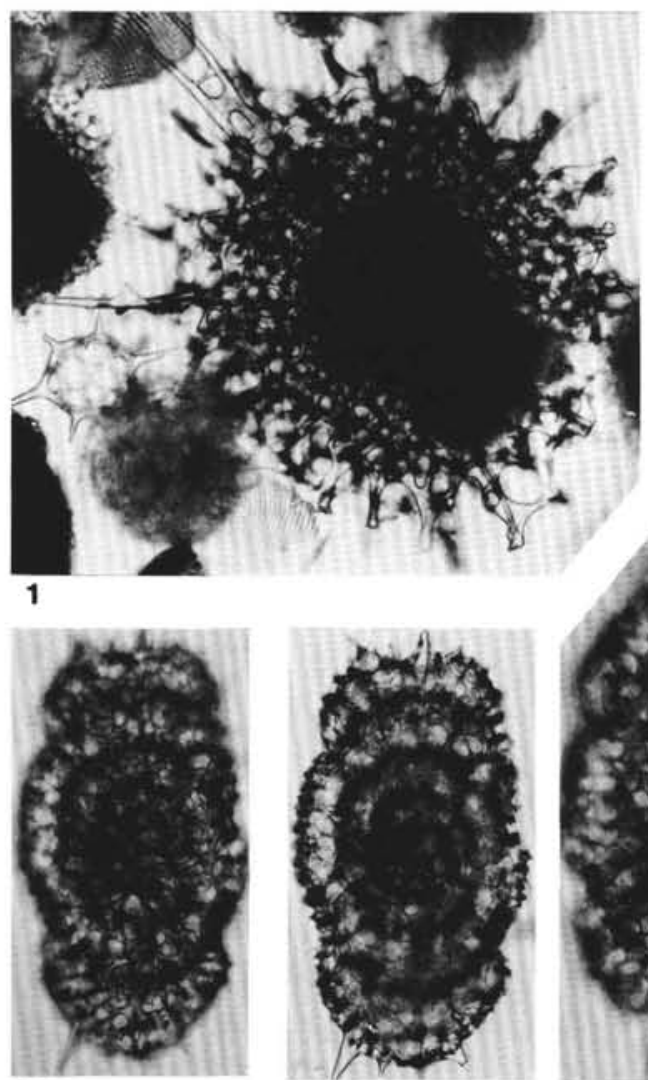

4

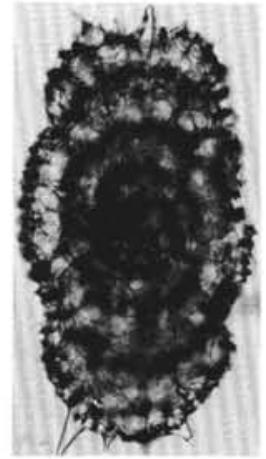

5

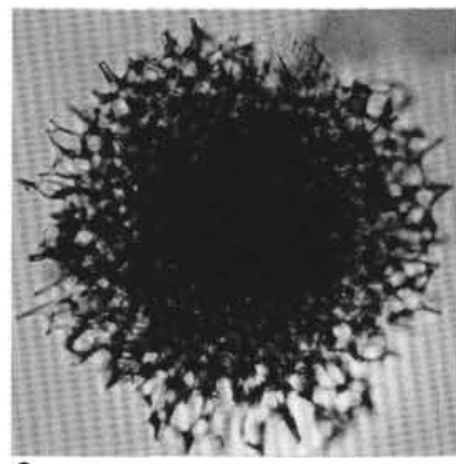

2

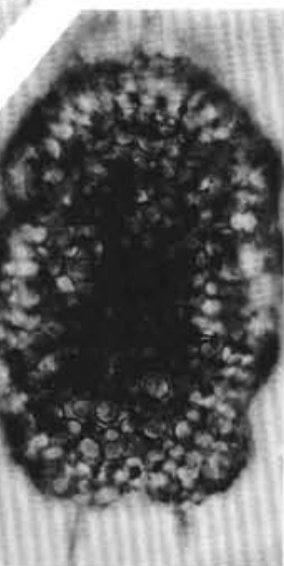

6

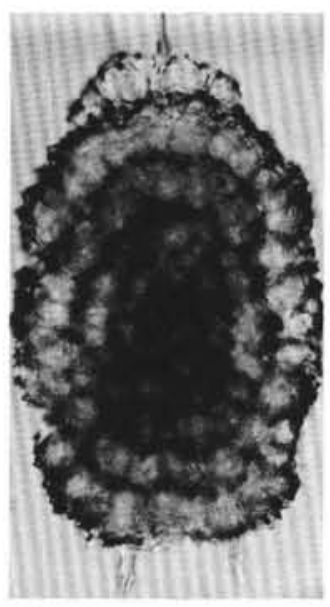

7

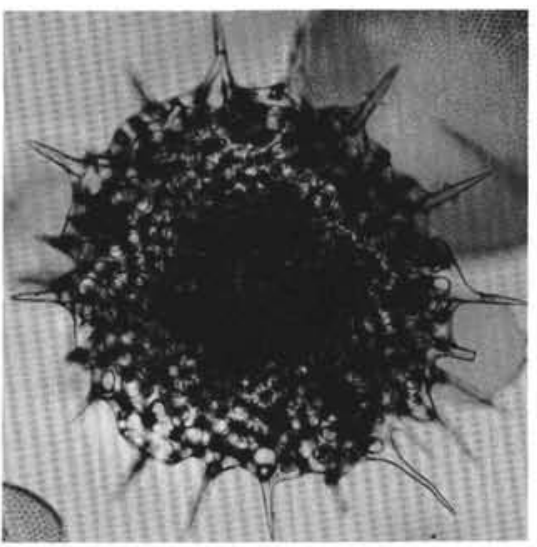

3
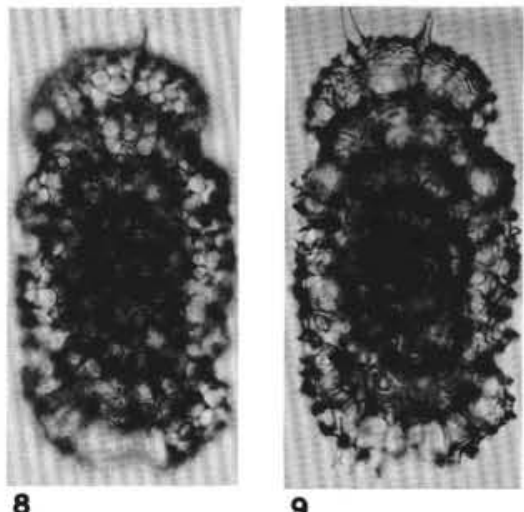

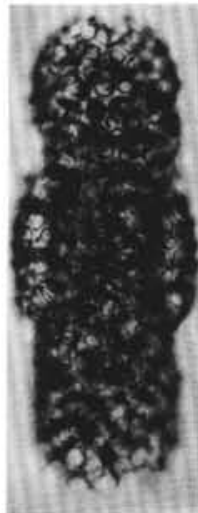

10

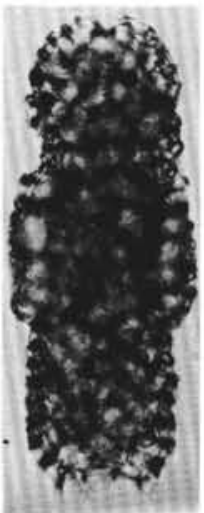

11

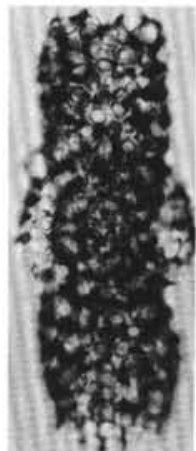

12

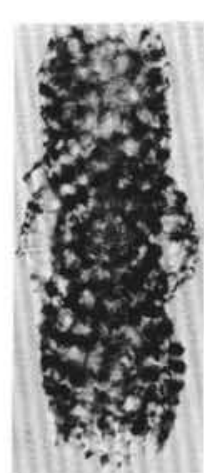

13

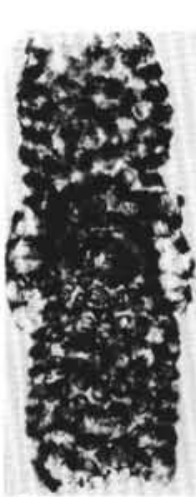

14

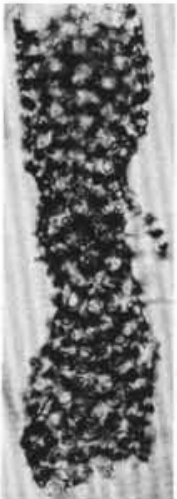

15

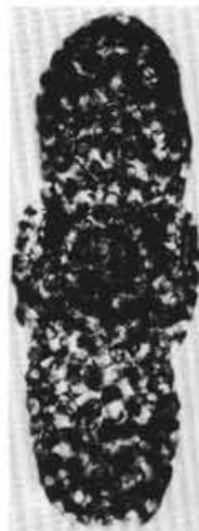

16

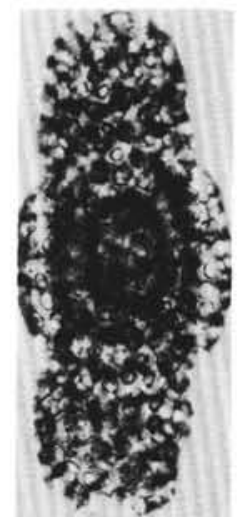

17

Plate 3. Scale bar $=100 \mu \mathrm{m}$. Figures 1-3. Spongotrochus vitabilis $\mathrm{n}$. sp. 1. Holotype; PMO 117.581; England finder T46/4; Sample 104-643A20X-3, 25-27 cm. 2. Sample 104-643A-20X-4, 25-27 cm. 3. Sample 104-643A-20X-3, 25-27 cm. Figures 4-9. Spongurus cauleti n. sp. 4, 5. Holotype; PMO 117.582; England Finder D16/1; Sample 104-642B-16H-2, 31-33 cm. 6-9. Sample 104-642B-16H-2, 31-33 cm. 6, 7 and 8, 9. England Finder Z8/0 and R51/3, respectively. Figures 10-17. Tessarastrum thiedei n. sp. 10, 11. Holotype; PMO 117.583; England Finder Z8/3; Sample 104-642B-13H-5, 24-26 cm. 12, 13. Sample 104-642B-13H-5, 64-66 cm. 14, 16, 17. Sample 104-642B-13H-5, 64-66 cm. 15. Sample $104-642 \mathrm{~B}-12 \mathrm{H}-3,75-77 \mathrm{~cm}$. 


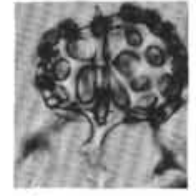

1

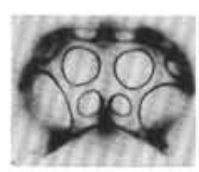

9

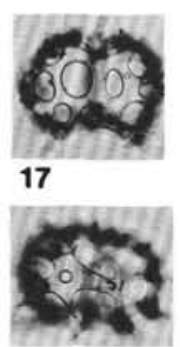

20

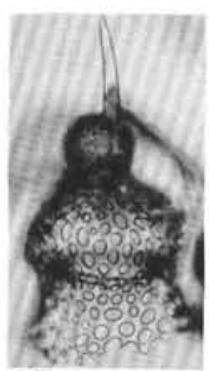

27

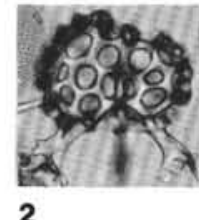

2

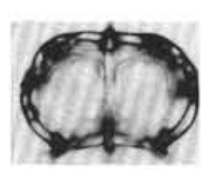

10

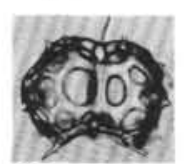

18

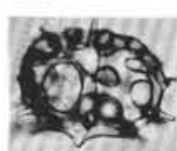

21

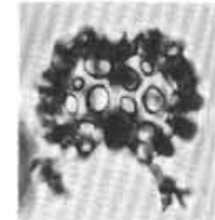

3

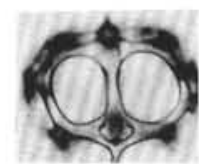

11

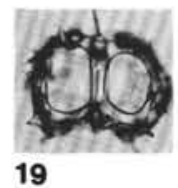

19

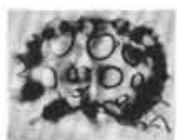

22

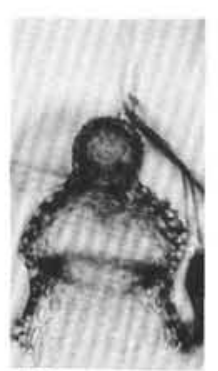

28

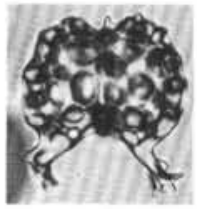

4

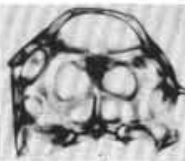

12

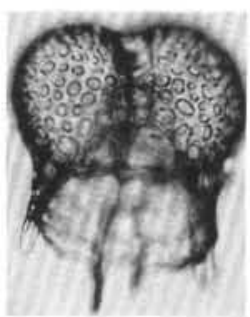

23

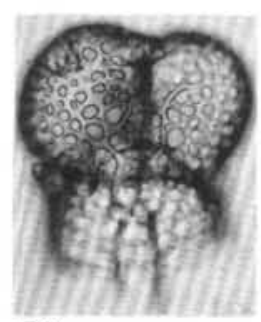

29

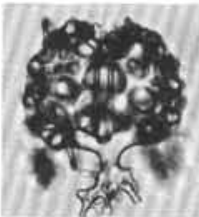

5

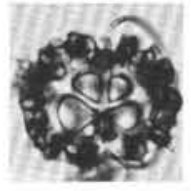

6

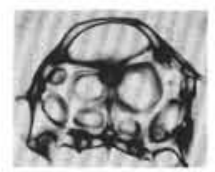

13

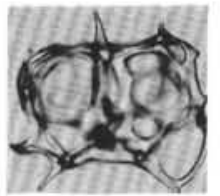

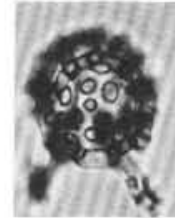

7

14

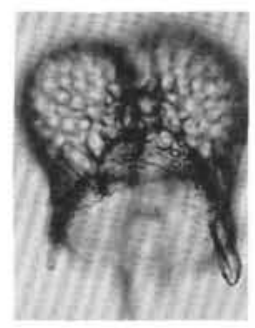

24

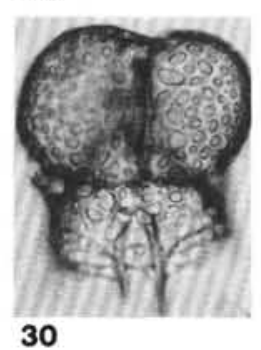

30

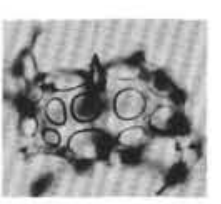

15

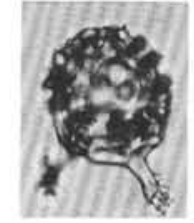

8

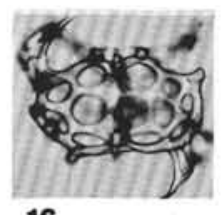

16

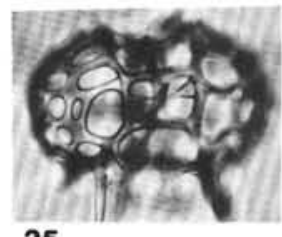

25

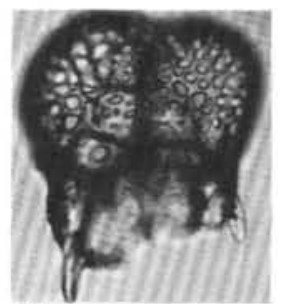

31

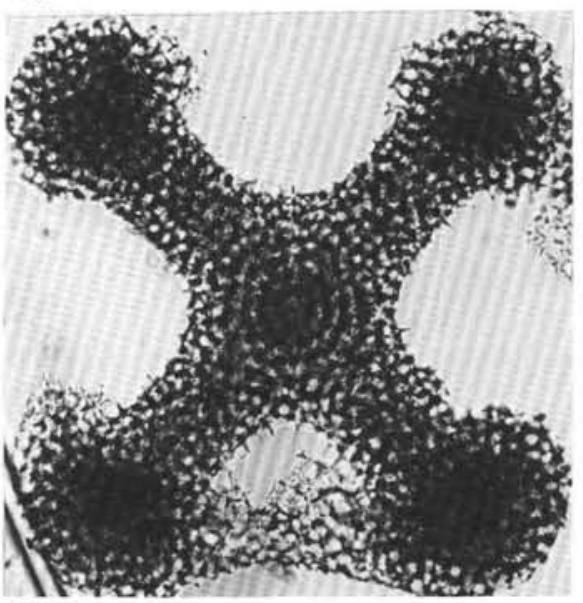

36
26

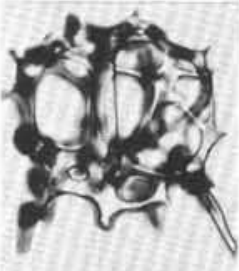

32

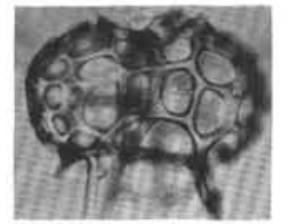

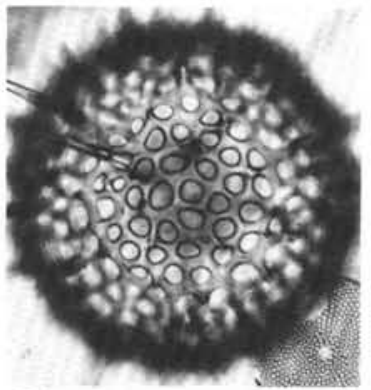

33

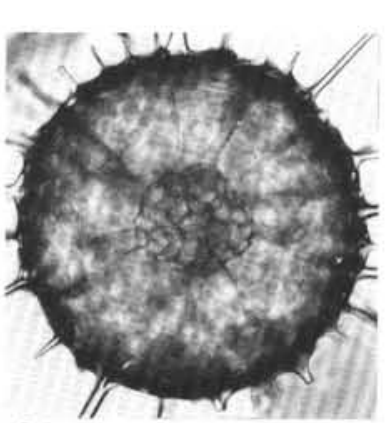

34

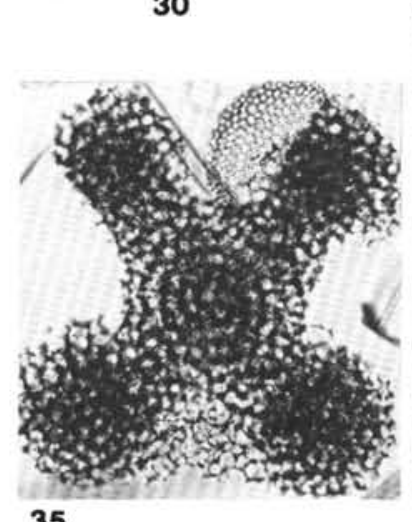

35

Plate 4. Scale bar $=100 \mu \mathrm{m}$. Figures 1-8. Corythosyris jubata sverdrupi n. subsp. 1, 2. Holotype; PMO 117.589; England Finder B20/3; Sample 104-643A-24X-3, 105-107 cm. 3-8. Different views of same specimen, Sample 104-643A-24X-3, 105-107 cm. Figures 9-16. Corythospyris reuschi n. sp. 9-11. Holotype; PMO 117.590; England Finder N48/2; Sample 104-642C-17H-2, 25-27 cm. 12, 13. Sample 104-642B-16H-7, 31$33 \mathrm{~cm}$. 14. Sample 104-642C-17H-3, $25-27 \mathrm{~cm}$. 15, 16. Sample $104-642 \mathrm{C}-17 \mathrm{H}-3,25-27 \mathrm{~cm}$. Figures 17-22. Corythospyris hispida n. sp. 1719. Holotype; PMO 117.588; England Finder T49/1; Sample 104-642C-23H-3, 24-27 cm. 20-22. Sample 104-642C-23H-5, 24-27 cm. Figures 23, 24, 29-31. Phormospyris thespios n. sp. 23, 24, 29, 30. Holotype;PMO 117.594; England Finder Z28/1; Sample 104-643A-24X-3, 105-107 cm. 31. Sample 104-642C-15H-6, 25-27 cm. Figures 25, 26, 32. Clathrospyris vogti $\mathrm{n}$. sp. 25, 26. Sample 104-643A-12H-1, 105-107 cm. 32. Holotype; PMO 117.587; England Finder E54/0; 104-643A-12H-1, 27-29 cm. Figures 27, 28. Gondwanaria japonica kiaeri n. subsp. Holotype; PMO 117.593; England Finder Z23/4; Sample 104-643A-29X-5, 105-107 cm. Figures 33, 34. Actinomma mirabile n. sp., 104-642B-20H-2, 20-22 cm. Figures 35-36. Spongaster tetras Ehrenberg, 1861. Sample 104-644A-31H-3, 25-27 cm. 


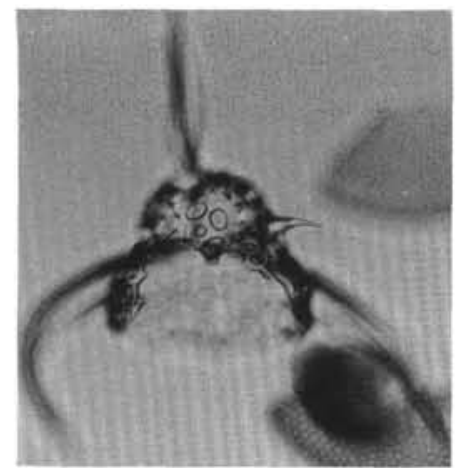

1

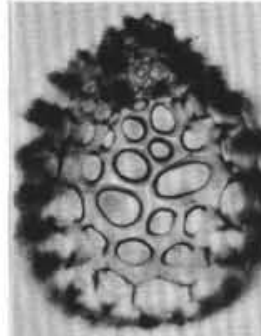

5

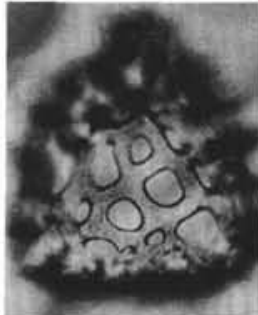

14

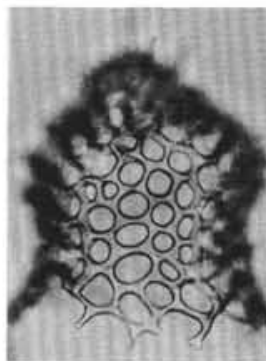

21

6

15

22
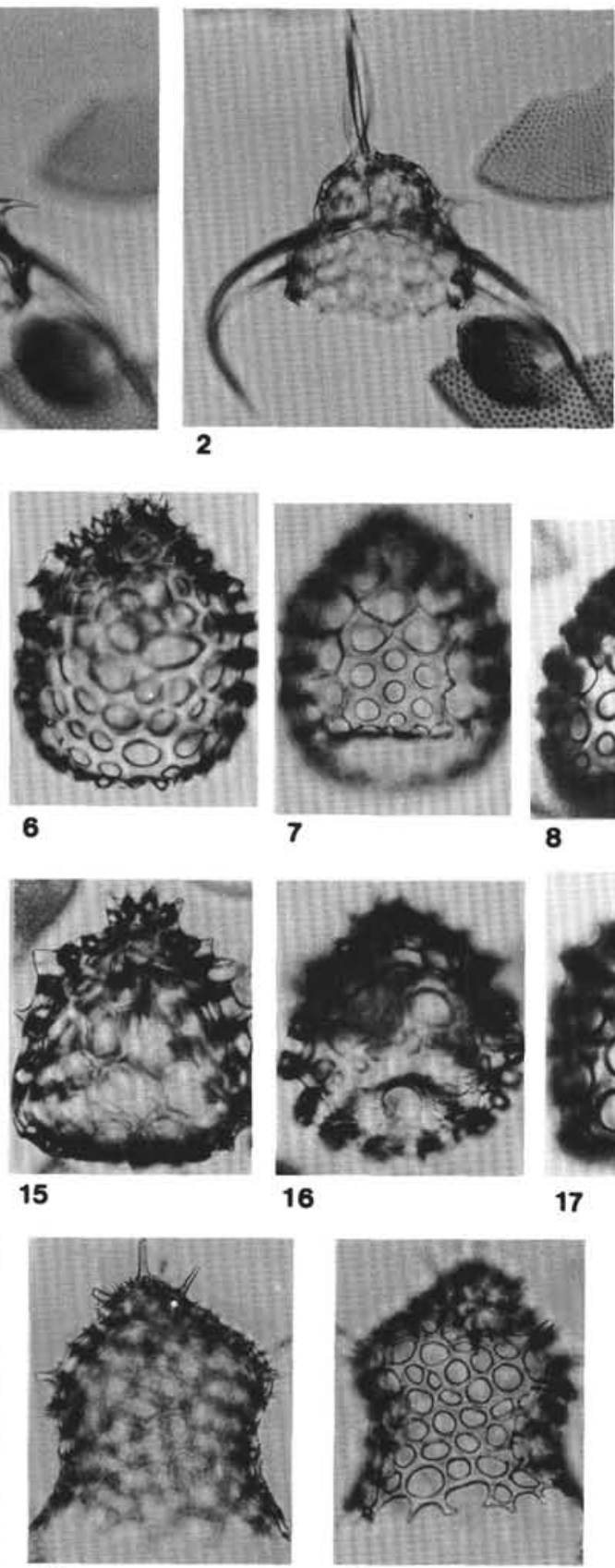

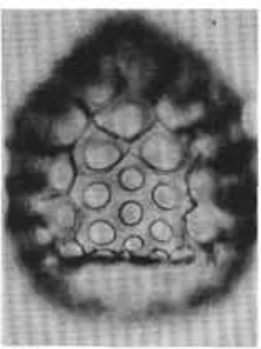

7

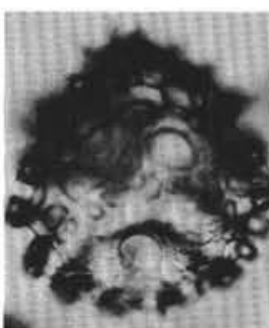

16

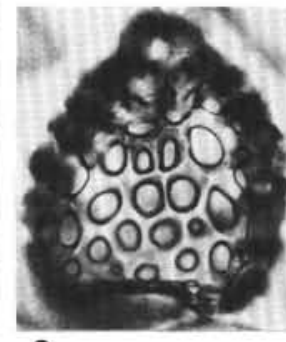

8

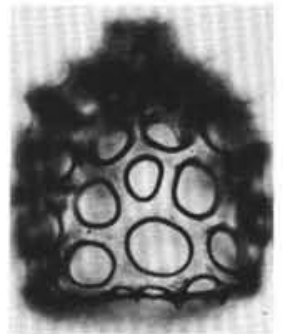

17

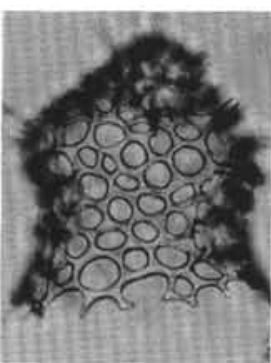

23

24

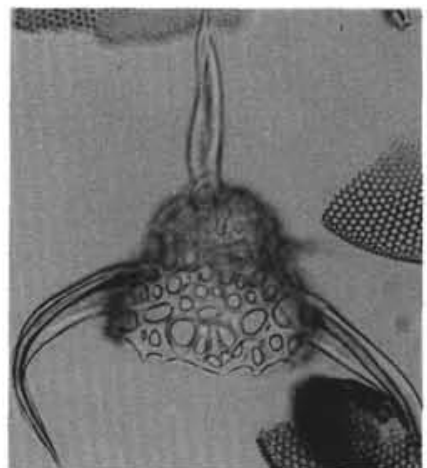

3

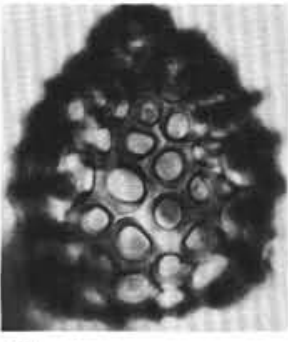

9

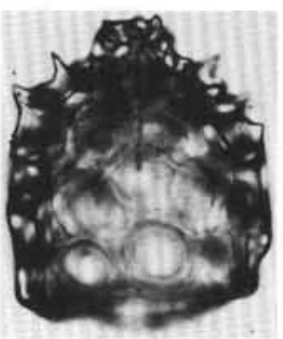

18

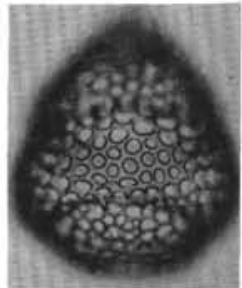

25

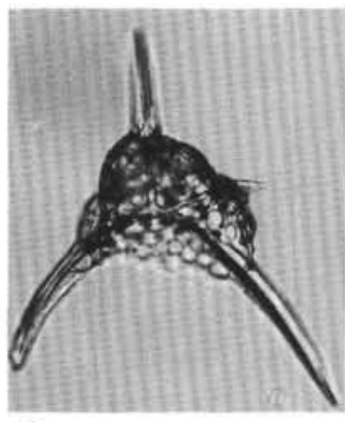

4

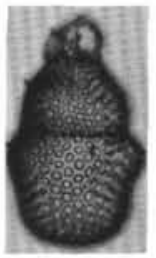

10

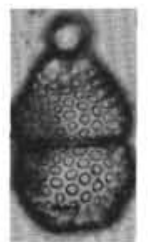

12

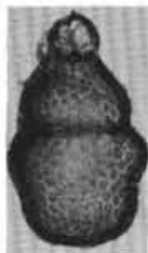

11

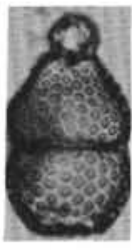

13

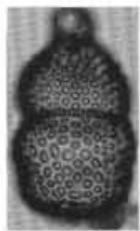

19

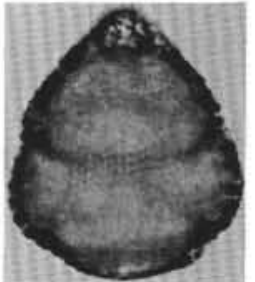

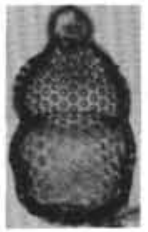

20

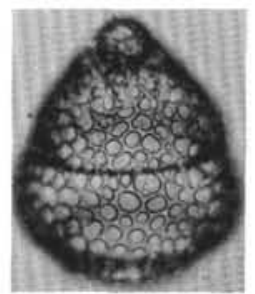

26

Plate 5. Scale bar $=100 \mu \mathrm{m}$. Figures 1-4. Pseudodictyophimus amundseni $\mathrm{n} . \mathrm{sp.}$ 1-3. Holotype; PMO 117.595; England Finder V23/1; Sample 104-643A-21X, CC. 4. Sample 104-643A-22X-2, 105-107 cm. Figures 5-9. Ceratocyrtis stoermeri n. sp. 5-7. Holotype; PMO 117.586; England Finder T49/0; Sample 104-642B-23H-1, 25-27 cm. 8, 9. Sample 104-642B-23H-1, 25-27 cm. Figures 10-13. Cyrtocapsella ampullacea n. sp. 10, 11. Holotype; PMO 117.592; England Finder E24/4; Sample 104-642C-22X, CC. 12, 13. Sample 104-642C-23H-3, 75-77 cm. England finder O52/4. Figures 14-18. Ceratocyrtis broeggeri n. sp. 14-16. Holotype; PMO 117.584; England Finder G54/2; Sample 104-643A-15H-3, $107-109 \mathrm{~cm}$. 17, 18. Sample 104-643A-15H-1, 105-107 cm. Figures 19, 20. Cyrtocapsella ampullacea $\mathrm{n}$. sp. Sample 104-642C-23H-3, 75-77 cm. England Finder K22/1. Figures 21-23. Ceratocyrtis manumi n. sp. Holotype; PMO 117.585; England Finder X25/4; Sample 104-643A-16H-3, 105-107 cm. Figures 24-26. Cyrtocapsella kladaros n. sp. Holotype; PMO 117.591; England Finder P54/0; Sample 104-643A-13H-5, 107-109 cm. 